\title{
Mitigating Elemental Imbalance
}

Heritable \& non-heritable adaptive responses to phosphorus limitation in an aquatic consumer

Kimberley Dianne Lemmen 
Thesis Committee: Prof. dr. M. Boersma

Prof. dr. E. Decaestecker

Dr. A. Derry

Prof. dr. G. A. Kowalchuck

Prof. dr. M. Winder

Prof. dr. J.C.M. Smeekens

Prof. dr. M.B. Soons

Design Cover: Margo Alleman Art

Printed by: Print Service Ede

ISBN: 978-90-831496-6-0

DOI: https://doi.org/10.33540/707

Thesis should be cited as:

Lemmen, Kimberley (2021) Mitigating elemental imbalance: Heritable \& nonheritable adaptive responses to phosphorus limitation in an aquatic consumer. PhD thesis. Utrecht University, the Netherlands 


\section{Mitigating Elemental Imbalance}

Heritable \& non-heritable adaptive responses to phosphorus limitation in an aquatic consumer

\section{Omgaan met stoichiometrische onevenwichten}

Erfelijke en niet-erfelijke manieren waarop een aquatische consument zich aanpast aan een gebrek aan fosfor

(met een samenvatting in het Nederlands)

\section{Proefschrift}

ter verkrijging van de graad van doctor aan de Universiteit Utrecht op gezag van de rector magnificus, prof.dr. H.R.B.M. Kummeling, ingevolge het besluit van het college voor promoties in het openbaar te verdedigen op

maandag 14 juni 2021 des middags te 4.15 uur

door

\section{Kimberley Dianne Lemmen}

geboren op 24 september 1988

te Edmonton, Canada 


\section{Promotor:}

Prof. dr. E. van Donk

\section{Copromotor:}

Dr. S.A.J. Declerck

This thesis is financially supported by grant $n^{\circ} 823.01 .011$ of the Earth and Life Sciences Division (ALW) of the Netherlands Organization for Scientific Research (NWO). 


\section{CONTENTS}

Chapter 1 General Introduction

Chapter 2 Stoichiometric traits vary widely within species: A metaanalysis of common garden experiments

Chapter 3 An experimental test of the Growth Rate Hypothesis as a predictive framework for microevolutionary adaptation

Chapter 4 Experimental evidence of rapid heritable adaptation in the absence of initial standing genetic variation

Chapter 5 Direct and indirect effects of phosphorus limitation on the functional response of the microzooplankton consumer Brachionus calyciflorus

Chapter 6 Synthesis

Chapter 7 References

Chapter 8

English Summary

Nederlandse samenvatting

Acknowledgements

About the Author

PE\&RC Certificate 



\section{Chapter 1}

General Introduction 

The last half century has been marked by tremendous progress in understanding ecological patterns and processes through first principles (e.g., coexistence theory, life history theory, resource ratio rule, metabolic theory). As the field matures, there is a growing need for ecologists to develop generalizable approaches for studying living systems that are applicable across scales (from cells to ecosystems) and can be used in a diversity of biota (Elser et al. 2000b). The ecological stoichiometry framework, which describes the balance and flow of elements within and between organisms, provides such an approach. While there has been an abundance of ecological research using stoichiometry, its use in a microevolutionary context remains limited. Given the unprecedented rate and magnitude of contemporary changes in elemental availability (Elser et al. 2009; Collins et al. 2013), understanding the trajectory of adaptive responses of populations to elemental imbalances becomes increasingly relevant. This thesis uses the rotifer Brachionus calyciflorus as a model system to understand how imbalances in the requirement and supply of a critical limiting element, phosphorus, affects primary consumers on timescales relevant to ecological processes.

\section{Ecological stoichiometry}

Stoichiometry is a concept in chemistry, dictated by the laws of physics, that allows for a quantitative description of the relationship between reactants and products of chemical reactions. A core principle of stoichiometry is the law of conservation of mass, which states that mass can neither be created or destroyed. Knowledge of the amount and ratio of elements in reactants allows for the prediction of the quantity of products, or vice versa. Reiners (1986), building on the work of Lotka (1925) and Redfield (1958), proposed using the principles of stoichiometry in an ecological context to study complex ecosystems. He suggested breaking down biological interactions into elemental building blocks and using the stoichiometry of organisms and their environment to understand patterns of biodiversity. For example, just as chemical reactions must be balanced, so too must consumerresource interactions. Ecological stoichiometry thus provides an integrative framework to study biotic and abiotic interactions by investigating the flow and relative abundances of elements in natural systems (Sterner \& Elser 2002). A focus on the elemental composition of organisms allows for better understanding of ecological processes and patterns such as nutrient cycling (Sterner et al. 1992; Moody et al. 2015), community composition (Hassett et al. 1997), and ecosystem function (Zechmeister-Boltenstern et al. 2015; Jochum et al. 2017).

One of the main challenges that organisms face is obtaining the elements necessary for survival, growth, and reproduction. The amount and relative abundances of elements required by an organism are determined by its structural components, metabolic functions, and reproductive efforts. Autotrophs, which are 
positioned at the base of the food web, obtain nutrients directly from the environment, and thus the uptake of separate elements is largely decoupled from each other. This aspect of their physiology, combined with the ability to store excess energy and nutrients, means that autotroph elemental composition is flexible and varies considerably depending on the environment (Figure 1). In contrast, heterotrophs, sitting higher on the food chain, take up nutrients in "packages" by consuming organic material (e.g., whole organisms) and have a relatively constrained elemental composition. Thus, circumstances often arise in which the elemental composition of a food resource differs greatly from what is required by the consumer, resulting in a stoichiometric mismatch (Sterner \& Elser 2002). This presents consumers with the challenge of acquiring the limiting element in sufficient quantities to support growth, while disposing of adequate amounts of excess elements to maintain the correct stoichiometric composition (Sterner \& Hessen 1994). Consumer fitness is maximized at a specific resource stoichiometry, and gradually decreases as the mismatch between consumers and their resources increases (Anderson \& Hessen 2005; Frost et al. 2006; Zhou \& Declerck 2019). Mechanisms to combat elemental imbalances include: altering ingestion rates, assimilation efficiency, and excretion (Plath \& Boersma 2001; Darchambeau et al. 2003; Suzuki-Ohno et al. 2012). Although these physiological responses serve to mitigate the fitness impact of stoichiometric imbalance between trophic levels, the extent to which they can compensate is limited (Burian et al. 2018).

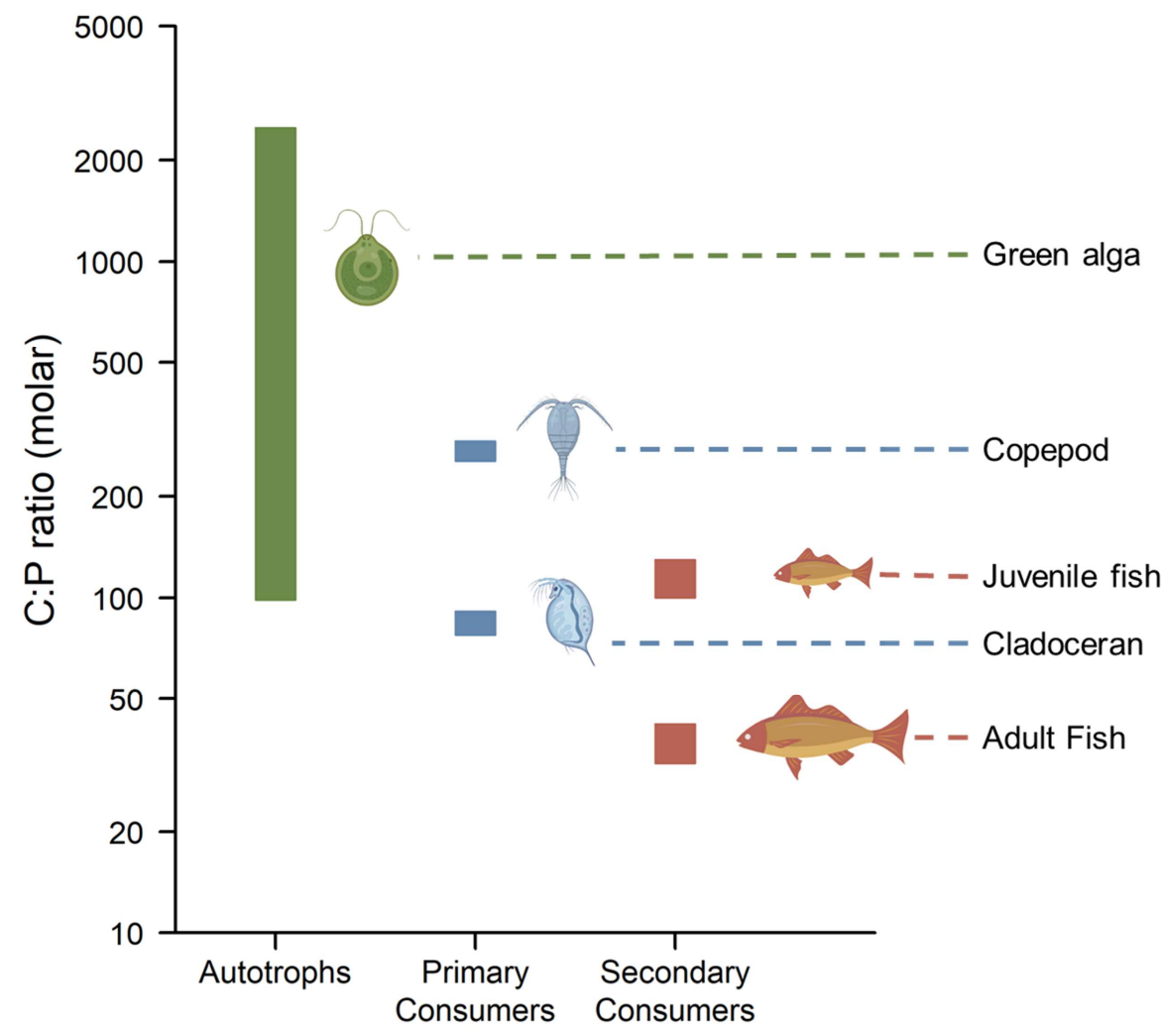

Figure 1. Carbon to phosphorus ratios $(\mathrm{C}: \mathrm{P})$ of autotrophs, primary consumers, and secondary consumers. Green algae display a wide range of relatively high $C: P$, whereas the $\mathrm{C}: \mathrm{P}$ of zooplankton and fish are typically low and considerably more constrained. Modified from van de Waal et al. (2010). 
While at least 31 elements are essential for bacterial, animal, or plant life (Williams \& Frausto da Silva 1997), ecological stoichiometry primarily focuses on carbon (C), nitrogen (N), and phosphorus (P) (Sterner \& Elser 2002). These three elements are the primary building blocks of biomolecules. Carbon forms the backbone of most organic molecules, and is particularly abundant in carbohydrates and lipids; $\mathrm{N}$ is abundant in proteins; and $\mathrm{P}$ is primarily found in phospholipids, energetic nucleotides (e.g., ATP), and nucleic acids (i.e., DNA and RNA). Carbon, N, and P dominate the elemental composition of living organisms, however, they represent only a small portion of the elements available on Earth's surface (Figure 2). As such, the availability of $\mathrm{C}, \mathrm{N}$, and $\mathrm{P}$ often constrains organismal growth and persistence, making the study of the flows and balances of these elements particularly important (also see Lind \& Jeyasingh (2018) and Rudman et al. (2019) for the role of microelements).

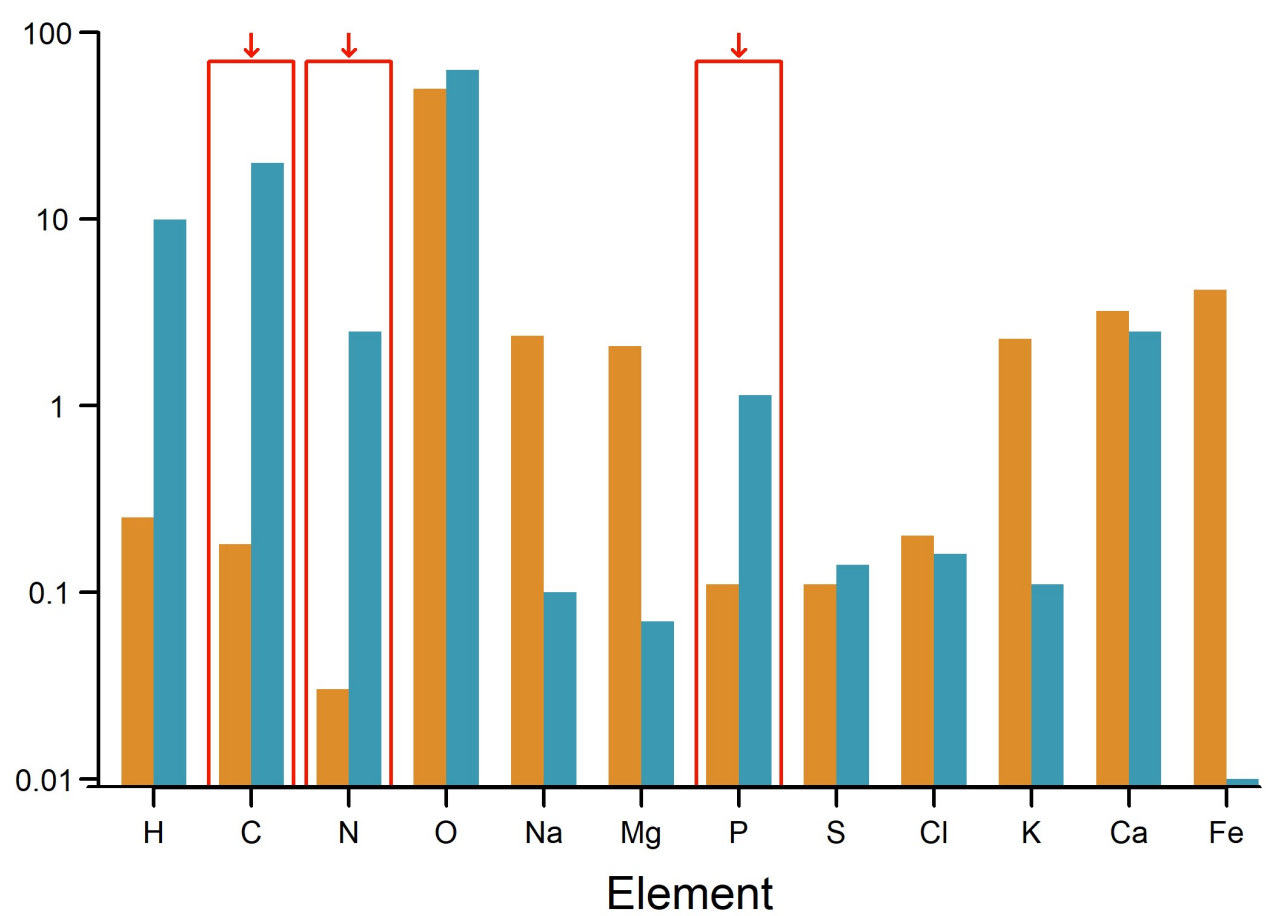

Figure 2. A comparison of the composition of the Earth's crust (yellow) and the human body (blue), for selected elements that dominate living organisms. Carbon, nitrogen, and phosphorus are generally disproportionately abundant in humans compared to what is available in the environment (highlighted by a red box). The y-axis is logarithmically scaled. Modified from Sterner and Elser (2002).

Phosphorus forms the backbone of nucleic acids and is thus essential for both general cellular maintenance and somatic growth. In many aquatic systems, as well as some terrestrial systems (Hou et al. 2020), $\mathrm{P}$ is considerably less abundant relative to other elements, and can be the limiting or a co-limiting element for primary production (Elser et al. 2007a). In such cases, P-limitation reduces primary and secondary productivity due to the high $\mathrm{P}$ requirement of nucleic acids (Elser et 
al. 1996). P-limitation of primary producers not only reduces organismal P-content, but it can also affect the biochemical composition (e.g., fatty acids and sterols [Müller-Navarra 1995; Boersma 2000; Piepho et al. 2010]) and physical properties (e.g., cell wall thickness [Tillberg et al. 1984]) of the organism. In primary consumers like zooplankton, the consumption of P-limited resources dramatically reduces growth rates (e.g., Elser et al. 2001; Seidendorf et al. 2010; Zhou et al. 2018). P-limitation also has the potential to impact higher trophic levels, and has been observed to negatively affect the body condition of secondary consumers (Malzahn et al. 2007; Boersma et al. 2008; Dickman et al. 2008). Changes in Pavailability have many, often cascading, effects on ecosystems, including community composition (Schulz \& Sterner 1999), nutrient cycling (Elser \& Urabe 1999), and the top-down control of herbivores (Burian et al. 2020). Given its importance for basic biological functions, studying the effect of $P$ on organismal life histories and ecosystem function has become a cornerstone of ecological stoichiometry.

\section{Ecological stoichiometry and evolution}

The advances made in understanding ecological patterns and processes using stoichiometry have led to the suggestion that this framework could also be useful in understanding evolution (Kay et al. 2005; Elser 2006). Natural selection shapes population genetic structure through the differential survival and reproductive success of phenotypes. However, identifying the factors responsible for phenotypic variation is difficult because environments are complex and multiple selective agents may be present (MacColl 2011; Wadgymar et al. 2017). Stoichiometry allows researchers to simplify the focal organism and their environment into elemental components to identify ecologically relevant mismatches and putative selective agents. Such an approach has been used with some success to describe macroevolutionary patterns. For example, total brain volume of different butterfly species shows a strong positive correlation with the $\mathrm{N}$ and sodium ( $\mathrm{Na}$ ) content of the plant species fed upon as larvae (Snell-Rood et al. 2020), suggesting that the availability of elements limits the number of neurons produced for a given body size. The relative abundance of elements has been linked to major evolutionary transitions such as the radiation of metazoan life that occurred during the Cambrian explosion (Elser et al. 2006), the evolution of omnivory (Diehl 2003), and contrasting life history strategies (Swanson et al. 2016).

The ecological stoichiometric framework has also been implemented to identify selective agents responsible for evolutionary changes within populations (i.e., microevolution). For example, increases in P-availability experienced by Daphnia during cultural eutrophication (i.e., transitioning from P-limited to P-replete) are suggested to be responsible for the evolution of less efficient $P$-use in low $P$ 
conditions (Frisch et al. 2014). In a similar adaptive response, increased barium (Ba) availability associated with the transition from marine to freshwater environments is suggested to have favoured a reduction in Ba uptake efficiency in freshwater threespine stickleback populations (Rudman et al. 2019). The relative availability of elements in the environment has also been implicated in microevolutionary changes to organismal stoichiometry within populations in a diversity of taxa, including bacteria (Turner et al. 2017), phytoplankton (Schlüter et al. 2014), and fish (Tobler et al. 2016). More research is needed to better understand the effect of elemental availability on microevolutionary trajectories.

The benefit of using ecological stoichiometry to study evolutionary processes is exemplified in its potential to describe eco-evolutionary dynamics. Repeated documentation that evolution can occur within a few generations (e.g., Hendry \& Kinnison 1999; Reznick \& Ghalambor 2001), and thus on ecologically relevant timescales (Hairston et al. 2005; Carroll et al. 2007), highlighted the need for integration between the two fields (reviewed in Hendry [2016]). It has been increasingly accepted that ecological and evolutionary processes interact, however, due to the complexity of natural systems, this has been difficult to empirically demonstrate. Ecological stoichiometry is ideal for studying ecoevolutionary dynamics due to the relative ease of tracing elemental movements between trophic and organizational levels (Matthews et al. 2011; Leal et al. 2017b). Furthermore, such an approach offers a unique perspective as the quantity and ratios of elements can act both as a selective pressure (e.g., resource quality) or as traits that respond to selection (e.g., elemental composition) (Kay et al. 2005; Elser 2006).

Stoichiometric mismatches between consumers and resources are suspected to be an important driver of eco-evolutionary feedbacks (Fussmann et al. 2007). When resources are stoichiometrically imbalanced, traits that increase the amount of the limiting element available for growth (e.g., increased P-use efficiency, elevated ingestion rates) will be favoured in consumer populations (Frisch et al. 2014; Burian et al. 2018). Such shifts in the elemental phenotype of consumers can alter population, community, and ecosystem interactions, which may in turn modify the selection environment of the consumer, resulting in an eco-evolutionary feedback. Changes in nutrient cycling in response to stoichiometric mismatches are one such trait shift that has the potential to influence eco-evolutionary dynamics (e.g., Jeyasingh et al. 2014; Kinnison et al. 2015). For example, if a resource is stoichiometrically imbalanced, consumers may alter the ratio at which the excess and limiting elements are excreted in (Vanni 2002; Burian et al. 2018) and reinforce the limitation of the depleted element (Sterner 1990), inducing a positive ecoevolutionary feedback (Schoener 2011). An investigation by Chowdhury \& 
Jeyasingh (2016) of Daphnia genotypes originating from P-limited and P-replete conditions suggests that such feedbacks are possible by demonstrating that algae co-occurring with $\mathrm{P}$-limited genotypes contained less recycled $\mathrm{P}$ and grew slower than algae with P-replete genotypes (Chowdhury \& Jeyasingh 2016).

There has been an abundance of theoretical research using ecological stoichiometry to understand eco-evolutionary dynamics (e.g., Matthews et al. 2011; Yamamichi et al. 2015; Branco et al. 2018), but few empirical investigations have been conducted. Experimental evolution studies provide important insights into the role of stoichiometric mismatch on eco-evolutionary dynamics and can help us to understand how organisms adapt to elemental limitation. For example, (Declerck et al. 2015) performed an evolution experiment with rotifers that selected for competitive ability when provided low- and high-P resources. They demonstrated that primary consumers adapted to P-limitation exert greater grazing pressure when feeding on P-limited resources than consumers adapted to P-replete conditions. The use of ecological stoichiometry to contextualize evolutionary responses, and their effects on ecological processes, represents a promising avenue of investigation.

\section{Incorporating ecological stoichiometry and microevolution to understand responses to environmental change}

Humans are dramatically altering the abundance and relative availability of elements on a global scale. Fossil fuel combustion and changes in land use (e.g., deforestation, fertilization) have led to greatly increased $\mathrm{CO}_{2}, \mathrm{CH}_{4}$, and $\mathrm{N}_{2} \mathrm{O}$ concentrations in the Earth's atmosphere (Galloway et al. 2004; Stocker et al. 2014). In addition, the mining of previously inaccessible $P$ reserves has dramatically intensified the input of $P$ to terrestrial, freshwater, and marine ecosystems (Smil 2000). Anthropogenic activities can also lead to decreases in elemental availability, as seen following the implementation of environmental regulations, that have resulted in significant reductions in P-loading in many freshwater systems (Stockner et al. 2000; Petri 2006; Tsai et al. 2014). Changes in the total and relative abundance of elements also differ spatially based on degree of human influence. For example, $\mathrm{N}$ deposition in highly impacted localities is almost twenty times greater than those free of human impact (Galloway et al. 2008).

Changes in biogeochemical cycles may lead to the amplification of stoichiometric mismatches between primary producers and their consumers (van de Waal et al. 2010). For example, the combined effects of increasing atmospheric $\mathrm{CO}_{2}$ concentrations, climate warming, and reductions in anthropogenic inputs of $\mathrm{N}$ and $\mathrm{P}$ have been observed to elevate autotroph $\mathrm{C}$ : nutrient ratios in lab and mesocosm 
experiments (Verschoor et al. 2013; Verspagen et al. 2014; Verbeek et al. 2018). While evolutionary responses in consumer populations may be able to mitigate the negative effects of elemental mismatch between trophic levels (Declerck et al. 2015), there have been limited applications of the ecological stoichiometric framework in a microevolutionary context to test this hypothesis.

\section{Rotifers as a study system}

Rotifers represent an important, often underappreciated, study system in biological research. The phylum Rotifera contains at least 2000 species with the shared characteristics of being small $(50-2,000 \mu \mathrm{m})$ and having an invertebrate body plan that is cylindrical, unsegmented, and bilaterally symmetrical (Wallace \& Snell 2010). Within the phylum there are two major groups: i) Monogonont rotifers, which display a diversity of life history strategies; and ii) Bdelloid rotifers, which reproduce exclusively by mitotic parthenogenesis (i.e., asexually). Both groups are primarily found in freshwater systems, however, some monogonont species live in saline environments and bdelloids can inhabit limnoterrestrial environments such as the water film on mosses and lichens. The diversity present within the rotifer phylum has been utilized to study fundamental biological questions such as the cost and benefits of sexual reproduction (Judson \& Normark 1996; Butlin 2002; Becks \& Agrawal 2012), the evolution of bet-hedging (Smith \& Snell 2012; FranchGras et al. 2017; Garcia-Roger et al. 2017; Tarazona et al. 2017), and the biology of aging (Snell 2014; Snell et al. 2018; Gribble \& Welch 2017). As rotifers are ubiquitous in freshwater systems and are important grazers, especially in eutrophic, fish-dominated water bodies (Guijun et al. 2012), ecological investigations of this group can aid our understanding of ecosystem function.

A defining characteristic of monogonont rotifer species is their cyclical parthenogenetic life cycle, combining sexual and asexual reproduction (Figure 3). The predominant mode of reproduction is asexual parthenogenesis, which allows the populations to grow quickly and generally occurs when environmental conditions are benign. Asexual females produce diploid offspring via apomictic parthenogenesis, which inhibits recombination (Birky \& Gilbert 1971), and therefore all descendants of a clonal line are genetically identical. Parthenogenic reproduction will occur until an environmental cue triggers an asexual female to produce sexual 'mictic' females (Schröder 2005). Unlike their asexual counterparts, the ooctyes of mictic females undergo meiosis, resulting in the production of recombinant haploid cells. Unfertilized haploid cells will develop into male offspring who then seek out and mate with mictic females. If a mictic female is fertilized it will produce a diploid diapausing embryo. These embryos may hatch in as little as 72 hours (Stelzer 2017), restarting the asexual phase of reproduction, however they can also accumulate in the sediment bank of the ecosystem to hatch 
at a later time (Langley et al. 2001; Lopes et al. 2016). Diapausing embryos are resistant to desiccation and, depending on the environmental conditions, may be viable for several decades (Marcus et al. 1994). The ability to alternate between reproductive modes allows for rapid genetic differentiation, and local adaptation of populations (De Meester 1996). Such microevolutionary change can occur because (i) the sexual phase of the life cycle generates recombinant genotypes upon which selection can act, and (ii) the asexual phase allows for rapid offspring production of genotypes with a fitness or stochastic advantage. Thus, multiple bouts of parthenogenic reproduction following sex allows for novel genotypes with high reproductive output to quickly dominate a population.

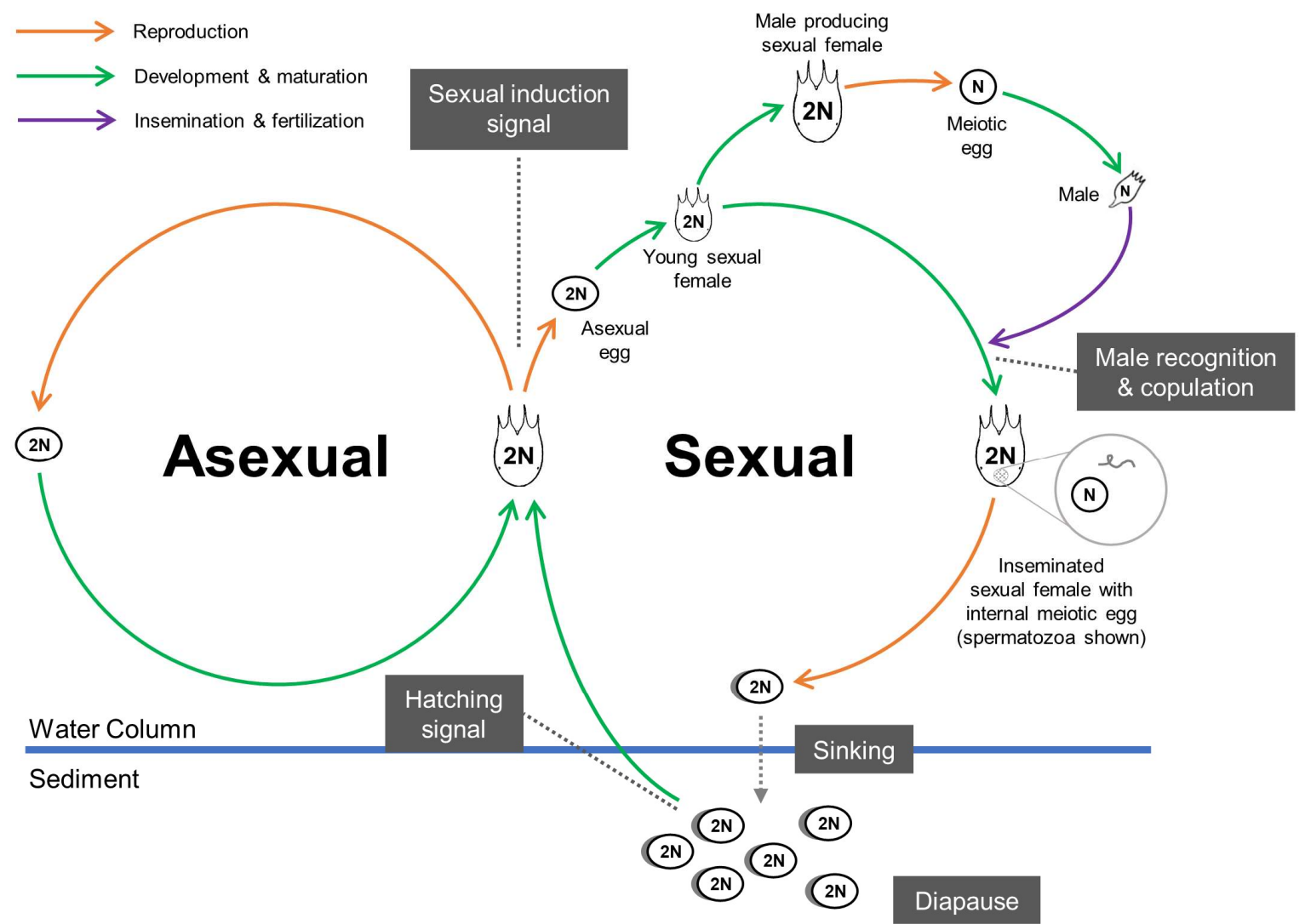

Figure 3. Cyclical parthenogenic life cycle of monogonont rotifers. Novel genotypes are produced through the hatching of diapausing eggs that are primarily found in lake sediments (although some float and remain in the water column). Hatchlings are diploid, asexual, and reproduce via apomictic parthenogenesis. The sexual phase of the life cycle is initiated by environmental factors (e.g., population density) at which point asexual mothers produce sexual daughters. Sexual females produce meiotic haploid eggs, if these eggs are not fertilized, they develop into haploid males. If sexual females are inseminated by a male, the meiotic egg is fertilized by the spermatozoa and produces a diploid diapausing egg. Diapausing eggs are resistant to harsh environmental conditions, such as drying, and may remain viable for decades. Hatching is induced by an environmental signal (e.g., temperature). Modified from Serra et al. (2019).

Monogonont rotifers are an excellent system to study contemporary evolution (Declerck \& Papakostas 2017). Their mode of reproduction allows for experimental 
investigations that are more difficult, and sometimes not possible, in obligate sexual organisms. The ability of rotifers to produce genetically identical offspring allows researchers to disentangle genetic and environmental effects on phenotype by comparing the response between genotypes to contrasting environmental conditions (Zhou et al. 2018). Additionally, diapausing embryos that have been deposited in a sediment bank enable genetic changes within a population to be tracked over time (Gómez \& Carvalho 2000; Burge et al. 2018). The small size of rotifers is also beneficial as it allows individuals to be easily manipulated during experiments. Being small also enables the maintenance of large populations in standard laboratory conditions, reducing the effect of drift during experiments without having to use large set ups under less controlled conditions (i.e., limnotrons, mesocosms). Finally, the short asexual and sexual generation time (Stelzer 2017; Zhou et al. 2018) allows evolution to occur on observable timescales. The capacity of rotifer populations to undergo rapid genetic differentiation, along with the ability to keep large replicate populations, make them an ideal metazoan system to perform experimental evolution studies (Declerck \& Papakostas 2017). Such studies are powerful tools to test theoretical predictions regarding adaptive trajectories (Kawecki et al. 2012). To date, thirteen experimental evolution studies have been conducted using monogonont rotifers (Table 1). 


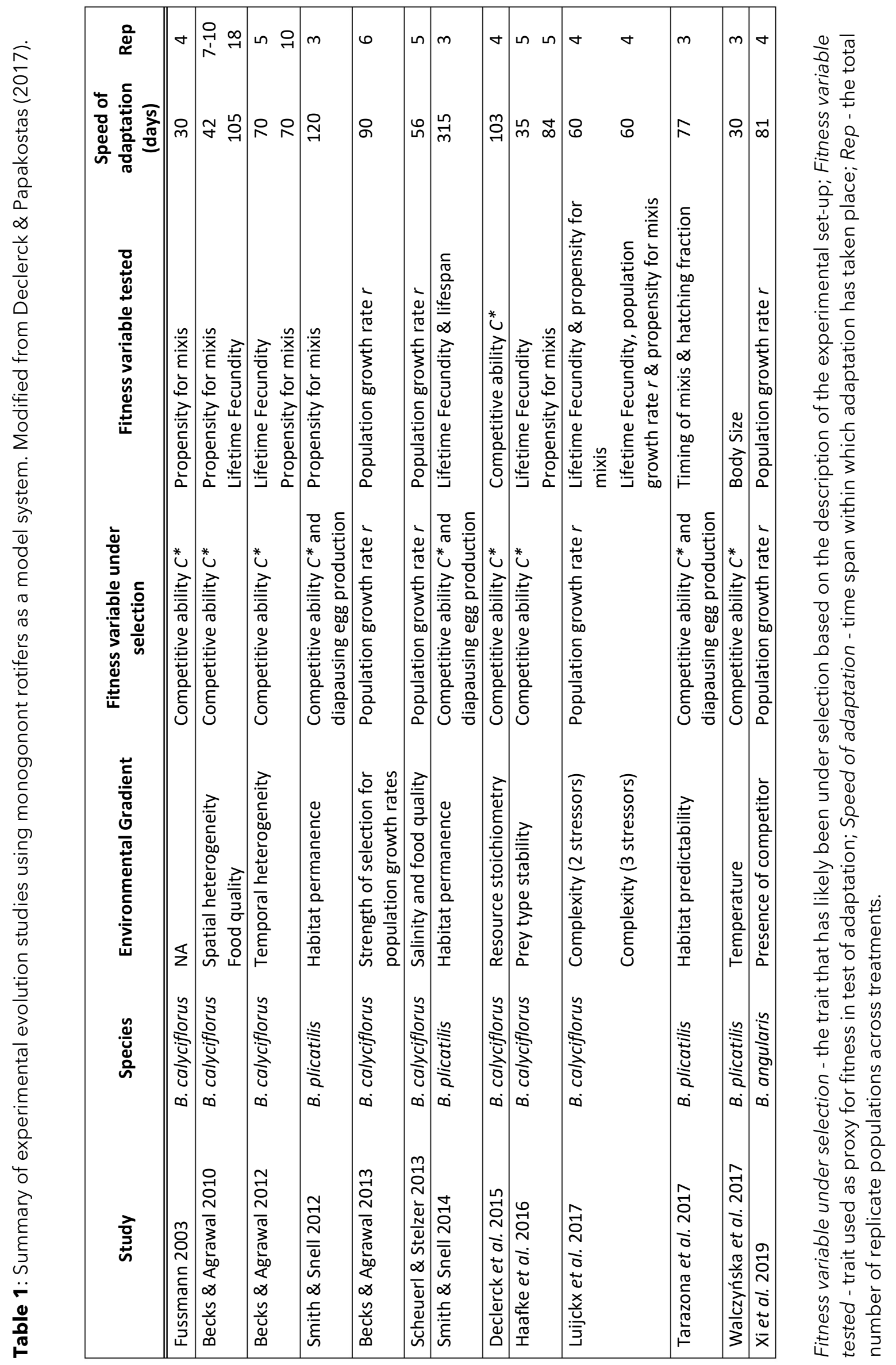




\section{Thesis outline (aims and research questions)}

This thesis aims to further incorporate an evolutionary perspective into the ecological stoichiometric framework. Specifically, I am interested in understanding how stoichiometric mismatches shape the phenotypes of primary consumers on ecologically relevant timescales. The rotifer Brachionus calyciflorus is used as a model system, taking advantage of its many beneficial traits to study ecological and evolutionary processes.

Intraspecific variation influences ecosystem processes and is essential for microevolutionary change. Although it has been acknowledged that stoichiometric traits (i.e., elemental composition, acquisition, assimilation, allocation, and excretion) are not fixed within a species, there has yet to be a comprehensive synthesis of the degree to which they vary, and how this variation differs among traits. To determine the extent of intraspecific variation, and thus the potential for stoichiometric traits to evolve, I performed a meta-analysis in Chapter 2. To ensure that measured trait variation reflected genetic differences, and not environmental factors, I collected data from studies that measured traits in a common garden environment. I additionally collected data on traits related to life history to assess how variation in stoichiometric traits compares to traits traditionally considered in a microevolutionary context.

Following confirmation that stoichiometric traits have the potential to evolve (Chapter 2), I investigated whether the ecological stoichiometric framework could be used to predict adaptive responses to selection (Chapter $\mathbf{3}$ ). This chapter uses the growth rate hypothesis, a key concept in ecological stoichiometry which posits that organismal $\mathrm{P}$ content is positively related to somatic growth rate, as a predictive framework for the effect of selection for fast growth on elemental composition. To test this prediction, I performed an evolution experiment with genetically diverse rotifer populations that were selected for fast population growth in environments with high and low phosphorus availability. I then evaluated evolutionary responses by rearing evolved and ancestral populations in a common garden experiment to compare population-level traits associated with fitness and P-stoichiometry.

Standing genetic variation is considered the mechanism by which rapid adaptive heritable responses occur (as seen in Chapter 3). Paradoxically, low diversity populations are common in nature and can be successful in the face of environmental change. Alternative pathways have been proposed as mechanisms for heritable adaptation, however experimental evidence is lacking to determine if, and to what extent, these sources of phenotypic variation can produce a response. Thus, in Chapter $\mathbf{4}$ I experimentally tested whether a heritable adaptive response 
can occur under conditions of stoichiometric mismatch in the absence of initial standing genetic variation. To investigate this question, I conducted a two-part experiment. First, I exposed monoclonal rotifer populations to a culturing regime that selected for phenotypic variants with elevated growth rates in environments with high and low phosphorus availability. Second, I performed two full factorial common garden experiments at different points in time during the exposure experiment to evaluate if observed adaptive responses were heritable.

Chapters 3 and $\mathbf{4}$ revealed that consumers can mitigate stoichiometric mismatch through genetic and possibly non-genetic adaptation. However, environments can deteriorate rapidly, and organisms may also have to rely on rapid plastic responses to adverse conditions. In Chapter 5, I investigated if consumers can modify their foraging behaviour in response P-limitation. In this study I explored how resource $P$-content impacted the ingestion rate of consumers using functional response models. I also tested whether the observed differences in ingestion rates between high and low phosphorus resources were the result of direct or indirect consequences of P-limitation.

Finally, Chapter $\mathbf{6}$ synthesizes the results of this thesis by drawing connections between the preceding chapters and discussing their main conclusions. I finish with a short outlook on prospective research directions. 



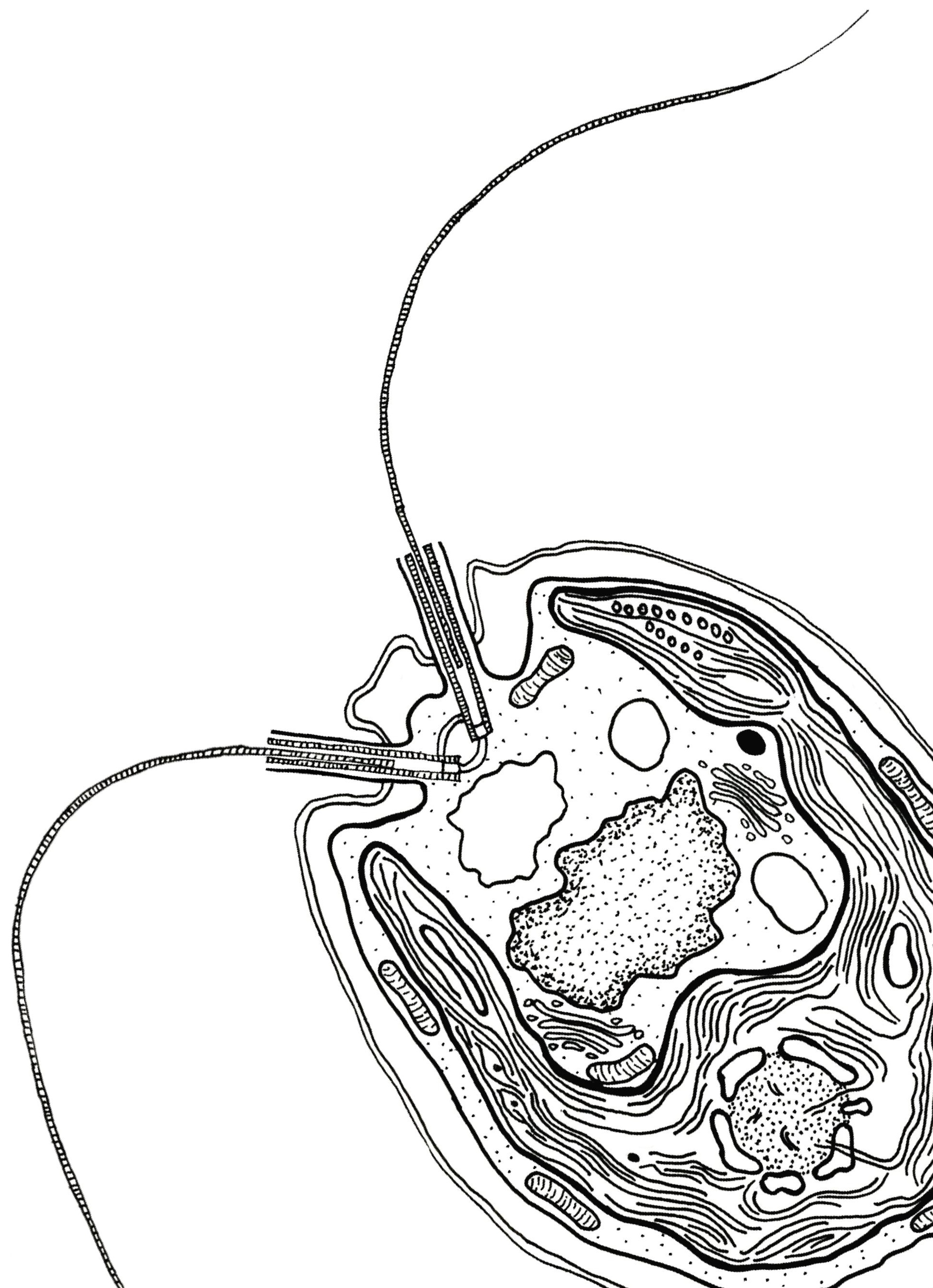




\section{Chapter 2}

Stoichiometric traits vary widely within species: A metaanalysis of common garden experiments

Kimberley D. Lemmen, Orpheus M. Butler, Thomas Koffel, Seth M.

Rudman, \& Celia C. Symons 


\section{Abstract}

'Ecological stoichiometry', a framework that focuses explicitly on the balances and flows of chemical elements within and between organisms and ecosystems, has provided crucial insights into many biological patterns and processes. Despite the proliferation of stoichiometrically-focused studies in recent decades and recognition of the potential for rapid evolution of stoichiometric traits, the prevalence of genetic variation in stoichiometric traits within species remains unclear. We compiled data from 30 published common garden studies of a broad range of taxa (including invertebrates, vertebrates and autotrophs) to examine how genetic variation influences the acquisition, assimilation, allocation (AAA), composition, and excretion of elements. To quantify the extent of genetic variation for a given trait we calculated the absolute mean response ratio from pairwise comparisons of populations within the same common garden (820 population and 708 genotype comparisons). We observed substantial intraspecific variation of stoichiometric traits across populations and among genotypes; however, the magnitude of variation was greater in AAA traits (effect sizes of $20 \%$ and $164 \%$ for population and genotype contrasts, respectively) and excretion (effect sizes of $52 \%$ and $23 \%$ ) than in content of carbon $(2.1 \%$ and $3.1 \%)$ and nitrogen $(4.5 \%$ and $24 \%)$. These results suggest that the content of some elements may be evolutionarily constrained relative to AAA traits that determine the processing of these elements, and that a sole focus on elemental content would underestimate the importance of intraspecific genetic variation, particularly within populations. Across many trait types the variation was greater among genotypes within a population than across populations. Finally, we compared pairs of populations from environments with different phosphorus $(P)$ availability to pairs of populations with similar $P$ availability. Genetic variation in the traits measured was similar regardless of the $P$ environment from which genotypes were isolated, suggesting that differences in elemental availability across environments do not necessarily drive enhanced trait divergence. Overall, our results highlight the substantial amount of intraspecific variation in stoichiometric traits and underscore the potential importance of intraspecific variation in driving ecological and evolutionary processes.

\section{Keywords}

elemental phenotype, evolution, eco-evolutionary dynamics, phosphorus, organismal stoichiometry, nutrient excretion, intraspecific variation 


\section{Introduction}

Ecological stoichiometry (ES) is a scientific framework that views living systems as composite chemical reactions which, like all physical processes, are governed by the law of mass balance (Sterner et al. 2015; Sterner \& Elser, 2002). In doing so, ES focuses explicitly on the balance and flow of elements and energy within and between organisms and ecosystems. The ES framework has applicability across a wide range of disciplines, from astrobiology to cancer research (Elser, 2003; Elser et al. 2007b), and can be applied across multiple levels of biological organization and across taxa. It has also been argued that explicit consideration of an organism's 'stoichiometric traits' (i.e., elemental contents and traits that influence the flux of elements between an organism and its environment) is a natural and convenient way to investigate the complex interplay between ecology and evolution (Kay et al. 2005). This is because elements can be traced through space and time, such that genetic variation in stoichiometric traits can be linked mechanistically to variation in environmental elemental availability (Leal et al. 2017b; Matthews et al. 2011).

Despite the apparent potential for stoichiometric traits to link ecology and evolution, there remain some fundamental gaps in our understanding of the genetic basis of variation in stoichiometric traits. In particular, while the composition, acquisition (A), assimilation (A), allocation (A), and excretion (E) of elements (hereafter referred to as 'elemental composition' and 'AAA', or 'AAAE') are regarded as the defining traits of the stoichiometric phenotype (Jeyasingh et al. 2014), it remains unclear how and to what extent genetic factors actually contribute to their variation within and between natural populations of conspecific organisms. Heritable trait variation is central to evolutionary processes, and high levels of intraspecific genetic trait variation within populations can be thought of as 'fuel' for adaptation (Barrett \& Schluter, 2008). At the same time, intraspecific genetic variation in stoichiometric traits has been identified as an important driver of variation in local ecological processes, such as leaf litter decomposition, that can scale up to affect overall ecosystem functioning (Silfver et al. 2007; Whitham et al. 2006). Quantification of the genetic component of intraspecific variation in stoichiometric traits can, therefore, help to identify which traits have the potential for contemporary evolution, and would represent an essential step toward the development of a stoichiometrically-explicit eco-evolutionary framework.

Intraspecific genetic variation in stoichiometric traits might emerge as a direct response to nutritionally-based or other selection pressures (Leal et al. 2017b), or because the stoichiometric traits are mechanistically correlated with other physiological, life history, and morphological traits, as in the well-documented coupling between $\mathrm{P}$ content and individual growth rate (the Growth Rate 
Hypothesis or ' $G R H^{\prime}$; see Elser et al. 2000a, 2003, 2008). However, stoichiometric traits also exhibit considerable plasticity, i.e., variability in response to environmental parameters (i.e., González et al. 2011; Townsend et al. 2007). For example, plant foliar nutrient content and resorption are often strongly correlated with soil nutrient availability (Rejmánková, 2005; Schade et al. 2003), and the elemental composition of invertebrates can be highly sensitive to the elemental composition of their diets (e.g., Visanuvimol \& Bertram, 2011; Zhou et al. 2018). Thus, in many prior studies that have considered intraspecific variation in stoichiometric traits, the respective genetic and environmental drivers of intraspecific variation have likely been confounded. However, as the field of ES has matured, an increasing number of studies have conducted 'common garden experiments' that can be used to assess the magnitude of intraspecific genetic variation in stoichiometric traits. These experiments minimize the effects of the environmental factors by comparing genetically distinct individuals within a common "garden" environment, thus allowing for the quantification of genetic variation either within or between populations (Lynch \& Walsh, 1998). Synthesis of such studies could illuminate whether genetic variation is present, the relative amount of genetic variation within and across populations, which stoichiometric traits show genetic variation, and whether elemental limitation could influence evolution in nature (Elser, 2006; Leal et al. 2017a; Rudman et al. 2019).

To determine the amount of intraspecific genetic variation in ES traits, we conducted a meta-analysis of studies that have measured ES traits on individuals reared in common gardens. These studies typically fall into two broad categories: those that compare ES traits between pairs of populations, and studies comparing ES traits of different genotypes from within a single population. From these studies we collected data on intraspecific genetic variation in ES traits, which we defined as measures of elemental content or movement, and life history data (specific growth rate, size/mass). We used these data to answer four questions about the extent of genetic variation in ES traits. 1) How much intraspecific genetic variation is present in ES traits relative to key life history traits? Here we used somatic growth rate and morphological characteristics to represent two key traits that are not explicitly stoichiometric, but have often been measured concurrently with ES traits. We predicted similar levels of genetic variation in life history traits and ES traits because all traits, at some level, have an elemental basis. 2) Do particular classes of ES traits show more genetic trait variation than others? We predicted that elemental composition would show reduced variation relative to AAAE traits, as there are well-documented constraints in elemental composition that are maintained by stoichiometric homeostasis (Sterner \& Elser, 2002). 3) Is the magnitude of intraspecific variation among genotypes (i.e., within a population) similar to that observed across populations? Individuals from different populations 
are often reproductively isolated and likely experience a more divergent environment than genotypes from a single population (Schluter, 2000). As such, we predicted a greater magnitude of genetic trait variation between distinct populations than among genotypes from within a population. 4) How does the magnitude of intraspecific genetic variation differ when comparing populations from distinct and similar stoichiometric environments? As resource stoichiometry has been demonstrated to be an important selection pressure (Jeyasingh \& Weider, 2007), we predict that populations from distinct stoichiometric environments will show greater genetic variation than populations from similar environments.

\section{Materials and Methods}

\section{Data Collection}

We collected studies for the meta-analyses by searching the ISI Web of Science ( $v$. 5.32) and Google Scholar databases with 'title' or 'abstract' search terms "("ecological stoichiometry" OR "biological stoichiometry") AND ("intraspecific variation" OR "evolution") AND "common garden"". Searches were conducted between the 15-17th of May 2019. These search terms produced 4 results in Web of Science and 197 results in Google Scholar. We screened these 201 studies for suitability based on their titles, abstracts, methods, and results. We then examined the reference lists of the seemingly-appropriate articles for additional studies that did not appear in our search results. We excluded studies that did not include data on ES traits. Thirty studies were eventually selected via this process. Importantly, this was not an exhaustive collation of the studies that might have met our criteria. This is presumably a result of our selection of search terms, which were necessarily broad in order to capture the full range of taxa, study systems, and ES traits throughout the literature without biasing our search toward particular combinations of these study foci. Thus, our collection of studies is perhaps best thought of as a large, reproducible sample of the literature with a bias toward studies conducted within the ES framework. We also note here that we explicitly excluded studies of genetic variation that was created by artificial selection (i.e., the crop and livestock literature) because the amount of genetic variation across treatments in artificial selection experiments will largely be proportional to the amount of standing variation included in the study and the strength and duration of artificial selection.

During study selection, it became clear that studies generally fell into two broad categories. Specifically, some studies reported stoichiometric trait variation within populations (i.e., variation among distinct genotypes), while others reported variation among distinct populations (i.e., variation in population trait means). We 
assigned each study to one of these categories (Table 1), and the categories were used as fixed effects variables as part of the meta-analysis.

Table 1. A complete list of the studies included in the meta-analysis, with information on study type - either populations ('P') or genotypes ('G'); the number of populations/genotypes ('\# Pop / Geno'), the type of ecosystem considered (Terrestrial, Freshwater or Marine); the coarse taxonomic group of the study organism (Plant, Invertebrate or Vertebrate); the Latin name of the study organism; for populations, if they were from biogeochemically distinct environments (always 'no' for genotypes); the number of common gardens in the experimental setup ('\# CGs'); the list of traits that were measured in the study ('Measured Trait'), and; the trait category under which they were grouped in the meta-analysis ('Response Variable', with 'SGR' standing for Somatic Growth Rate).

\begin{tabular}{|c|c|c|c|c|c|c|c|c|c|}
\hline Study & $\begin{array}{c}\text { Study } \\
\text { type }\end{array}$ & $\begin{array}{c}\text { \# Pop / } \\
\text { Geno }\end{array}$ & Ecosystem & Group & Species & $\begin{array}{l}\text { Biochem } \\
\text { distinct? }\end{array}$ & $\begin{array}{c}\# \\
\text { CGs }\end{array}$ & $\begin{array}{c}\text { Measured } \\
\text { Trait } \\
\end{array}$ & $\begin{array}{l}\text { Response } \\
\text { Variable }\end{array}$ \\
\hline $\begin{array}{l}\text { Barbour et } \\
\text { al. (2015) }\end{array}$ & G & 26 & Terrestrial & Plant & $\begin{array}{l}\text { Salix } \\
\text { hookeriana }\end{array}$ & No & 1 & $\begin{array}{l}\mathrm{N} \text { content } \\
\mathrm{C} \text { content } \\
\mathrm{C}: \mathrm{N} \\
\text { Body length }\end{array}$ & $\begin{array}{l}\text { N content } \\
\text { C content } \\
\text { C:N } \\
\text { Morphology }\end{array}$ \\
\hline $\begin{array}{l}\text { Chowdhury } \\
\text { \& Jeyasingh } \\
\text { (2016) }\end{array}$ & $P$ & 2 & Freshwater & Invert. & $\begin{array}{l}\text { Daphnia } \\
\text { pulicaria }\end{array}$ & Yes & 2 & $\begin{array}{l}\text { P content } \\
\text { P net } \\
\text { incorporation }\end{array}$ & $\begin{array}{l}\text { P content } \\
\text { AAA }\end{array}$ \\
\hline $\begin{array}{l}\text { Chowdhury } \\
\text { et al. } \\
\text { (2014) }\end{array}$ & G & 2 & Freshwater & Invert. & Daphnia pulex & No & 2 & $\begin{array}{l}\text { P retention } \\
\text { efficiency } \\
\text { C retention } \\
\text { efficiency } \\
\text { P excretion } \\
\text { C excretion }\end{array}$ & Excretion \\
\hline $\begin{array}{l}\text { Crutsinger } \\
\text { et al. } \\
\text { (2009) }\end{array}$ & G & 12 & Terrestrial & Plant & $\begin{array}{l}\text { Solidago } \\
\text { altissima }\end{array}$ & No & 1 & $\begin{array}{l}\mathrm{N} \text { content } \\
\mathrm{C} \text { content } \\
\mathrm{C}: \mathrm{N} \\
\text { Decomp rate }\end{array}$ & $\begin{array}{l}\mathrm{N} \text { content } \\
\mathrm{C} \text { content } \\
\mathrm{C}: \mathrm{N} \\
\text { Excretion }\end{array}$ \\
\hline $\begin{array}{l}\text { Crutsinger } \\
\text { et al. } \\
\text { (2014b) }\end{array}$ & G & 5 & Terrestrial & Plant & $\begin{array}{l}\text { Populus } \\
\text { trichocarpa }\end{array}$ & No & 1 & $\begin{array}{l}\mathrm{P} \text { content } \\
\mathrm{N} \text { content } \\
\mathrm{C} \text { content } \\
\mathrm{C}: \mathrm{P} \\
\mathrm{C}: \mathrm{N} \\
\text { Body length }\end{array}$ & $\begin{array}{l}\text { P content } \\
\mathrm{N} \text { content } \\
\mathrm{C} \text { content } \\
\mathrm{C}: \mathrm{P} \\
\mathrm{C}: \mathrm{N} \\
\text { Morphology }\end{array}$ \\
\hline $\begin{array}{l}\text { Crutsinger } \\
\text { et al. } \\
(2014 a)\end{array}$ & G & 2 & Terrestrial & Plant & $\begin{array}{l}\text { Baccharis } \\
\text { pilularis }\end{array}$ & No & 1 & $\mathrm{C}: \mathrm{N}$ & C:N \\
\hline $\begin{array}{l}\text { Dalton et } \\
\text { al. (2017) }\end{array}$ & $P$ & 4 & Freshwater & Vert. & $\begin{array}{l}\text { Poecilia } \\
\text { reticulata }\end{array}$ & No & 2 & $\begin{array}{l}N \text { content } \\
\text { C content } \\
\text { P content } \\
\text { C:P } \\
\text { C:N } \\
N: P \\
N \text { excretion } \\
\text { P excretion } \\
N: P \text { excretion } \\
\text { SGR }\end{array}$ & $\begin{array}{l}N \text { content } \\
C \text { content } \\
P \text { content } \\
C: P \\
C: N \\
N: P \\
\text { Excretion }\end{array}$ \\
\hline $\begin{array}{l}\text { Declerck et } \\
\text { al. (2015) }\end{array}$ & $P$ & 2 & Freshwater & Invert. & $\begin{array}{l}\text { Brachionus } \\
\text { calyciflorus }\end{array}$ & Yes & 2 & $\begin{array}{l}\text { C content } \\
\text { P content } \\
C: P \\
\text { Use } \\
\text { efficiency }\end{array}$ & $\begin{array}{l}\text { C content } \\
\text { P content } \\
\text { C:P } \\
\text { AAA }\end{array}$ \\
\hline
\end{tabular}




\begin{tabular}{|c|c|c|c|c|c|c|c|c|c|}
\hline Study & $\begin{array}{c}\text { Study } \\
\text { type }\end{array}$ & $\begin{array}{c}\text { \# Pop / } \\
\text { Geno }\end{array}$ & Ecosystem & Group & Species & $\begin{array}{l}\text { Biochem } \\
\text { distinct? }\end{array}$ & $\begin{array}{c}\# \\
\text { CGs } \\
\end{array}$ & $\begin{array}{l}\text { Measured } \\
\text { Trait } \\
\end{array}$ & $\begin{array}{l}\text { Response } \\
\text { Variable } \\
\end{array}$ \\
\hline $\begin{array}{l}\text { DeMott et } \\
\text { al. (2004) }\end{array}$ & $\bar{P}$ & 2 & Freshwater & Invert. & $\begin{array}{l}\text { Daphnia } \\
\text { dentifera }\end{array}$ & Yes & 1 & P content & P content \\
\hline $\begin{array}{l}\text { Dinh Van et } \\
\text { al. (2013) }\end{array}$ & $P$ & 3 & Freshwater & Invert. & $\begin{array}{l}\text { Ischnura } \\
\text { elegans }\end{array}$ & No & 6 & $\begin{array}{l}\text { Zn content } \\
\text { SGR }\end{array}$ & $\begin{array}{l}\text { Zn content } \\
\text { SGR }\end{array}$ \\
\hline $\begin{array}{l}\text { Espeset et } \\
\text { al. (2019) }\end{array}$ & $P$ & 2 & Terrestrial & Invert. & Pieris rapae & Yes & 2 & $\begin{array}{l}\mathrm{N} \text { content } \\
\mathrm{C} \text { content } \\
\mathrm{C}: \mathrm{N} \\
\text { Mass }\end{array}$ & $\begin{array}{l}\mathrm{N} \text { content } \\
\mathrm{C} \text { content } \\
\mathrm{C}: \mathrm{N} \\
\text { Morphology }\end{array}$ \\
\hline $\begin{array}{l}\text { Frisch et al. } \\
\text { (2014) }\end{array}$ & $P$ & 4 & Freshwater & Invert. & $\begin{array}{l}\text { Daphnia } \\
\text { pulicaria }\end{array}$ & Yes & 2 & $\begin{array}{l}\text { P retention } \\
\text { efficiency } \\
\text { P use } \\
\text { efficiency } \\
\text { SGR }\end{array}$ & AAA \\
\hline $\begin{array}{l}\text { Güsewell, } \\
\text { Jakobs \& } \\
\text { Weber } \\
(2006)\end{array}$ & $P$ & 2 & Terrestrial & Plant & $\begin{array}{l}\text { Solidago } \\
\text { gigantea }\end{array}$ & No & 1 & $\begin{array}{l}\mathrm{N} \text { content } \\
\mathrm{C} \text { content } \\
\mathrm{P} \text { content } \\
\mathrm{N} \text { mineraliz. } \\
\mathrm{P} \text { mineraliz. } \\
\text { Decomp rate } \\
\text { Mass }\end{array}$ & $\begin{array}{l}\text { N content } \\
\text { C content } \\
\text { P content } \\
\text { AAA } \\
\text { Excretion } \\
\text { Morphology }\end{array}$ \\
\hline $\begin{array}{l}\text { Hughes, } \\
\text { Stachowicz } \\
\& \text { Williams } \\
(2009)\end{array}$ & G & 8 & Marine & Plant & Zostera marina & No & 1 & $\begin{array}{l}\mathrm{N} \text { uptake rate } \\
\text { Mass }\end{array}$ & $\begin{array}{l}\text { AAA } \\
\text { Morphology }\end{array}$ \\
\hline $\begin{array}{l}\text { Jeyasingh } \\
\text { et al. } \\
\text { (2009) }\end{array}$ & G & 6 & Freshwater & Invert. & $\begin{array}{l}\text { Daphnia } \\
\text { pulicaria }\end{array}$ & No & 2 & $\begin{array}{l}\text { C content } \\
\text { P content }\end{array}$ & $\begin{array}{l}\text { C content } \\
\text { P content }\end{array}$ \\
\hline $\begin{array}{l}\text { Jeyasingh } \\
\text { et al. } \\
\text { (2015) }\end{array}$ & $P$ & 2 & Freshwater & Invert. & Daphnia pulex & No & 2 & $\begin{array}{l}\text { P content } \\
\text { P excretion } \\
\text { P retention } \\
\text { efficiency } \\
\text { C retention } \\
\text { efficiency } \\
\text { P uptake rate } \\
\text { C uptake rate } \\
\text { SGR }\end{array}$ & $\begin{array}{l}\text { P content } \\
\text { Excretion } \\
\text { AAA }\end{array}$ \\
\hline $\begin{array}{l}\text { Johnsen et } \\
\text { al. (2009) }\end{array}$ & G & 39 & Terrestrial & Plant & $\begin{array}{l}\text { Oenothera } \\
\text { biennis }\end{array}$ & No & 1 & $\begin{array}{l}\mathrm{C}: \mathrm{N} \\
\text { Mass }\end{array}$ & $\begin{array}{l}\text { C:N } \\
\text { Morphology }\end{array}$ \\
\hline $\begin{array}{l}\text { Leal et al. } \\
\text { (2017a) }\end{array}$ & $P$ & 2 & Freshwater & Vert. & $\begin{array}{l}\text { Gasterosteus } \\
\text { aculeatus }\end{array}$ & No & 1 & $\begin{array}{l}\text { P content } \\
\mathrm{N}: \mathrm{P} \\
\text { P excretion } \\
\text { Condition } \\
\text { Body length }\end{array}$ & $\begin{array}{l}\text { P content } \\
\mathrm{N}: \mathrm{P} \\
\text { Excretion } \\
\text { Morphology }\end{array}$ \\
\hline $\begin{array}{l}\text { LeRoy et al. } \\
\text { (2007) }\end{array}$ & G & 4 & Terrestrial & Plant & $\begin{array}{l}\text { Populus } \\
\text { backcross }\end{array}$ & No & 1 & $\begin{array}{l}\mathrm{N} \text { content } \\
\mathrm{P} \text { content } \\
\mathrm{C}: \mathrm{N}\end{array}$ & $\begin{array}{l}\mathrm{N} \text { content } \\
\mathrm{P} \text { content } \\
\mathrm{C}: \mathrm{N}\end{array}$ \\
\hline $\begin{array}{l}\text { LeRoy et al. } \\
\text { (2007) }\end{array}$ & G & 5 & Terrestrial & Plant & $\begin{array}{l}\text { Populus } \\
\text { F1_hybrid }\end{array}$ & No & 1 & $\begin{array}{l}\mathrm{N} \text { content } \\
\mathrm{P} \text { content } \\
\mathrm{C}: \mathrm{N}\end{array}$ & $\begin{array}{l}\mathrm{N} \text { content } \\
\mathrm{P} \text { content } \\
\mathrm{C}: \mathrm{N}\end{array}$ \\
\hline $\begin{array}{l}\text { LeRoy et al. } \\
\text { (2007) }\end{array}$ & G & 5 & Terrestrial & Plant & $\begin{array}{l}\text { Populus } \\
\text { fremontii }\end{array}$ & No & 1 & $\begin{array}{l}\mathrm{N} \text { content } \\
\mathrm{P} \text { content } \\
\mathrm{C}: \mathrm{N}\end{array}$ & $\begin{array}{l}\mathrm{N} \text { content } \\
\mathrm{P} \text { content } \\
\mathrm{C}: \mathrm{N}\end{array}$ \\
\hline $\begin{array}{l}\text { LeRoy et al. } \\
\text { (2007) }\end{array}$ & G & 5 & Terrestrial & Plant & $\begin{array}{l}\text { Populus } \\
\text { angustifolia }\end{array}$ & No & 1 & $\begin{array}{l}\mathrm{N} \text { content } \\
\mathrm{P} \text { content } \\
\mathrm{C}: \mathrm{N}\end{array}$ & $\begin{array}{l}\mathrm{N} \text { content } \\
\mathrm{P} \text { content } \\
\mathrm{C}: \mathrm{N}\end{array}$ \\
\hline
\end{tabular}




\begin{tabular}{|c|c|c|c|c|c|c|c|c|c|}
\hline Study & $\begin{array}{c}\text { Study } \\
\text { type }\end{array}$ & $\begin{array}{c}\text { \# Pop / } \\
\text { Geno } \\
\end{array}$ & Ecosystem & Group & Species & $\begin{array}{l}\text { Biochem } \\
\text { distinct? }\end{array}$ & $\begin{array}{c}\# \\
\text { CGs } \\
\end{array}$ & $\begin{array}{l}\text { Measured } \\
\text { Trait } \\
\end{array}$ & $\begin{array}{l}\text { Response } \\
\text { Variable } \\
\end{array}$ \\
\hline $\begin{array}{l}\text { LeRoy, } \\
\text { Wooley \& } \\
\text { Lindroth } \\
\text { (2012) }\end{array}$ & G & 5 & Terrestrial & Plant & $\begin{array}{l}\text { Populus } \\
\text { tremuloides }\end{array}$ & No & 2 & $\begin{array}{l}\text { C content } \\
N \text { content } \\
C: N\end{array}$ & $\begin{array}{l}\text { C content } \\
N \text { content } \\
C: N\end{array}$ \\
\hline $\begin{array}{l}\text { Liess et al. } \\
(2013)\end{array}$ & $P$ & 2 & Freshwater & Vert. & $\begin{array}{l}\text { Rana } \\
\text { temporaria }\end{array}$ & No & 2 & $\begin{array}{l}\text { P content } \\
\text { C content } \\
\mathrm{N} \text { content } \\
\text { Mass } \\
\text { SGR }\end{array}$ & $\begin{array}{l}\text { P content } \\
\mathrm{C} \text { content } \\
\mathrm{N} \text { content } \\
\text { Morphology } \\
\text { SGR }\end{array}$ \\
\hline $\begin{array}{l}\text { Liess et al. } \\
(2015)\end{array}$ & $P$ & 2 & Freshwater & Vert. & $\begin{array}{l}\text { Rana } \\
\text { temporaria }\end{array}$ & No & 4 & $\begin{array}{l}\text { P excretion } \\
N \text { excretion } \\
\text { Mass } \\
\text { SGR }\end{array}$ & $\begin{array}{l}\text { Excretion } \\
\text { Morphology } \\
\text { SGR }\end{array}$ \\
\hline $\begin{array}{l}\text { Madritch \& } \\
\text { Hunter } \\
(2005) \\
\end{array}$ & G & 9 & Terrestrial & Plant & Quercus laevis & No & 1 & $\begin{array}{l}\mathrm{C} \text { content } \\
\mathrm{N} \text { content } \\
\mathrm{C}: \mathrm{N}\end{array}$ & $\begin{array}{l}\mathrm{C} \text { content } \\
\mathrm{N} \text { content } \\
\mathrm{C}: \mathrm{N}\end{array}$ \\
\hline $\begin{array}{l}\text { Madritch et } \\
\text { al. (2006) }\end{array}$ & G & 5 & Terrestrial & Plant & $\begin{array}{l}\text { Populus } \\
\text { tremuloides }\end{array}$ & No & 2 & $\begin{array}{l}\mathrm{C} \text { content } \\
\mathrm{N} \text { content } \\
\mathrm{C}: \mathrm{N} \\
\text { Decomp rate }\end{array}$ & $\begin{array}{l}\text { C content } \\
\mathrm{N} \text { content } \\
\mathrm{C}: \mathrm{N} \\
\text { Excretion }\end{array}$ \\
\hline $\begin{array}{l}\text { Moody et } \\
\text { al. (2018) }\end{array}$ & $P$ & 9 & Freshwater & Vert. & $\begin{array}{l}\text { Gambusia } \\
\text { marshi }\end{array}$ & Yes & 2 & $\begin{array}{l}\mathrm{N} \text { content } \\
\mathrm{C} \text { content } \\
\mathrm{P} \text { content } \\
\mathrm{C}: \mathrm{N} \\
\mathrm{C}: \mathrm{P} \\
\mathrm{N}: \mathrm{P} \\
\mathrm{N} \text { excretion } \\
\mathrm{P} \text { excretion } \\
\mathrm{N}: \mathrm{P} \text { excretion } \\
\text { Mass }\end{array}$ & $\begin{array}{l}N \text { content } \\
\text { C content } \\
\text { P content } \\
\mathrm{C}: \mathrm{N} \\
\mathrm{C}: \mathrm{P} \\
\mathrm{N}: \mathrm{P} \\
\text { Excretion } \\
\\
\text { Morphology }\end{array}$ \\
\hline $\begin{array}{l}\text { Neiman et } \\
\text { al. (2012) }\end{array}$ & $P$ & 2 & Freshwater & Invert. & $\begin{array}{l}\text { Potamopyrgus } \\
\text { antipodarum }\end{array}$ & No & 2 & $\begin{array}{l}\text { P content } \\
\text { SGR }\end{array}$ & $\begin{array}{l}\text { P content } \\
\text { SGR }\end{array}$ \\
\hline $\begin{array}{l}\text { Prater et al. } \\
\text { (2017) }\end{array}$ & $P$ & 4 & Freshwater & Invert. & $\begin{array}{l}\text { Daphnia } \\
\text { pulicaria }\end{array}$ & Yes & 3 & $\begin{array}{l}\text { P content } \\
\text { P use } \\
\text { efficiency } \\
\text { SGR }\end{array}$ & $\begin{array}{l}\text { P content } \\
\text { AAA } \\
\text { SGR }\end{array}$ \\
\hline $\begin{array}{l}\text { Prater et al. } \\
\text { (2017) }\end{array}$ & $P$ & 8 & Freshwater & Invert. & $\begin{array}{l}\text { Daphnia } \\
\text { mendotae }\end{array}$ & Yes & 3 & $\begin{array}{l}\text { P content } \\
\text { P use } \\
\text { efficiency } \\
\text { SGR }\end{array}$ & $\begin{array}{l}\text { P content } \\
\text { AAA } \\
\text { SGR }\end{array}$ \\
\hline $\begin{array}{l}\text { Rudman et } \\
\text { al. (2019) }\end{array}$ & G & 2 & Freshwater & Vert. & $\begin{array}{l}\text { Gasterosteus } \\
\text { aculeatus }\end{array}$ & No & 1 & $\begin{array}{l}\text { Zn content } \\
\text { P content }\end{array}$ & $\begin{array}{l}\text { Zn content } \\
\text { P content }\end{array}$ \\
\hline $\begin{array}{l}\text { Rudman et } \\
\text { al. (2019) }\end{array}$ & $P$ & 2 & Freshwater & Vert. & $\begin{array}{l}\text { Gasterosteus } \\
\text { aculeatus }\end{array}$ & Yes & 2 & $\begin{array}{l}\text { Zn content } \\
\text { P content } \\
\text { P uptake rate }\end{array}$ & $\begin{array}{l}\text { Zn content } \\
\text { P content } \\
\text { AAA }\end{array}$ \\
\hline $\begin{array}{l}\text { Sherman et } \\
\text { al. (2017) }\end{array}$ & G & 10 & Freshwater & Invert & $\begin{array}{l}\text { Daphnia } \\
\text { pulicaria }\end{array}$ & No & 2 & $\begin{array}{l}\text { P content } \\
\text { P acquisition } \\
\mathrm{C} \text { acquisition } \\
\text { P assimilation } \\
\text { C assimilation } \\
\text { P net } \\
\text { incorporation } \\
\text { C net } \\
\text { incorporation } \\
\text { P retention } \\
\text { efficiency } \\
\text { SGR }\end{array}$ & $\begin{array}{l}\text { P content } \\
\text { AAA }\end{array}$ \\
\hline
\end{tabular}




\begin{tabular}{lcclllllll}
\hline Study & $\begin{array}{c}\text { Study } \\
\text { type }\end{array}$ & $\begin{array}{c}\text { \# Pop / } \\
\text { Geno }\end{array}$ & Ecosystem & Group & Species & $\begin{array}{l}\text { Biochem } \\
\text { distinct? }\end{array}$ & $\begin{array}{l}\text { \# } \\
\text { CGs }\end{array}$ & $\begin{array}{l}\text { Measured } \\
\text { Trait }\end{array}$ & $\begin{array}{l}\text { Response } \\
\text { Variable }\end{array}$ \\
\hline \hline Trakimas et & $\mathrm{P}$ & 3 & Terrestrial & Invert & Gryllus integer & No & 1 & C content & C content \\
al. (2019) & & & & & & & N content & N content \\
& & & & & & & C:N & C:N \\
& & & & & & Resting & AAA \\
& & & & & & metabolic & rate \\
\end{tabular}

For each study we recorded details of location, ecosystem type, focal taxa, the purported reasons for differentiation between populations and all measured stoichiometric traits. For a given trait to be included in our analyses, it was necessary that at least two studies reported common garden data about that trait. We considered traits to be explicitly 'stoichiometric' if they were part of the elemental phenotype defined by Jeyasingh et al. (2014) as elemental composition, acquisition, assimilation, allocation, and excretion (CAAAE). Many organismal traits, including growth rate and body size, are often closely coupled with CAAAE traits in such ways that could justify their categorisation as a 'stoichiometric trait' if defined broadly. By adopting a strict definition of stoichiometric trait, we are able to qualitatively compare the intraspecific genetic variation of unambiguously stoichiometric traits with life history traits that do not explicitly involve the movement of elements.

Due to differences in how often CAAAE traits have been measured we found it necessary to subdivide or group certain traits. We considered the content of carbon $(C)$, nitrogen $(N)$ and phosphorus $(P)$ and their ratios as separate traits due to the abundant reporting of elemental composition. Few studies measured acquisition, assimilation, or allocation, therefore we grouped these aspects of the elemental phenotype into a single trait category (AAA) for all elements. We also recorded somatic growth rate and measures of mass and size as 'morphological traits' because they were the most commonly measured life history traits for comparison. Studies on invertebrates and vertebrates measured and reported elemental composition of whole organisms, while studies on plants used leaf tissues. In studies on plants, we included data from studies that measured either green or senesced vegetation as elemental content and data on the decomposition rate of plant material, which has modest heritability (RodriguezCabal et al. 2017), was included as excretion (Table 1).

Means, variances and sample sizes for traits were extracted from repositories, tables, figures, or text. Standard deviation was estimated from SE, 95\% confidence intervals or quantiles using standard methods (Bland, 2015; Higgins \& Green, 2008). We used DataThief III (Tummers, 2006) to extract data from figures. Where data were not provided in a format suitable for our analyses, we contacted study authors via email to request the relevant data. In cases where study designs 
featured more than one type of common garden (e.g., Dinh Van et al. 2013), population contrasts within different common gardens were treated as unique observations. For example, if there were two common gardens, and population $A$ and population $B$ were both raised in both common gardens, then there were two population contrasts included in our meta-analysis dataset, one for each common garden.

\section{Effect size calculation}

For each trait within a study, we compared the difference in genotype/population's trait values by calculating the log response ratios (LRR) for all pairwise combinations of genotypes/populations grown in the same common garden.

$$
\begin{gathered}
L R R=y_{i}=\ln \left(\frac{\overline{\text { Tralt }}_{1}}{\text { Tralt }_{2}}\right) \\
\text { variance } L R R=v_{i}=\frac{S D_{1}{ }^{2}}{n_{1}\left(\overline{\text { Tralt }}_{1}\right)^{2}}+\frac{S D_{2}{ }^{2}}{n_{2}\left(\text { Tralt }_{2}\right)^{2}}
\end{gathered}
$$

where $i$ is the study, $\overline{\operatorname{Tralt}}_{j}$ is the genotype/population mean, $S D_{j}$ the standard deviation and $n_{j}$ is the sample size (number of replicates), with $j=1,2$ corresponding to the two genotypes/populations being compared. We choose to use LRR because (i) it is dimensionless, allowing for comparison between studies with different methodologies and units, (ii) it is easily interpretable as it quantifies the proportionate change (Hedges et al. 1999), and (iii) the value of the LRR is not affected by non-independent samples (Noble et al. 2017). We defined $n_{j}$ differently for studies comparing genotypes and those comparing populations. For genotype studies, $n_{j}$ was the number of individuals measured for a given genotype. For population studies, $n_{j}$ was the number of genetically distinct individuals measured for a given population. For example, in a sexually reproducing species $n_{j}$ is the number of individuals measured, but in studies of cyclical parthenogens (e.g., Daphnia) $n_{j}$ is the number of distinct clone lines measured. If it was not clearly indicated that genetically different individuals were included in the study, then $n_{j}$ was recorded as 1.

In the set of studies that compared traits of different genotypes from the same population, we were not always able to use all pairwise comparisons to calculate LRRs. This group of studies often had a larger number of genotypes to be compared than studies comparing distinct populations (maximum number of genotypes compared was 39, and populations was 9), and the use of all pairwise comparisons was too computationally demanding. Instead, if a common garden measured less than five genotypes we calculated the LRR for all pairwise 
combinations. However, if six or more genotypes were measured (six studies), pairwise comparisons were performed for only five genotypes (min, first quartile, median, third quartile, max). With this method we took a subset of the data to be computationally reasonable and to represent the full range of trait values. We also used a conservative approach and calculated all LRRs within a common garden using the median trait value as the denominator in equation 1 (see Supplementary Material). Although the magnitude and $95 \%$ credibility interval of some mean effect estimates of these two methods differed, it did not change the interpretation of the data. In the studies that compared mean traits between populations, the maximal number of distinct populations was small enough to enable us to compute all possible pairwise comparison, even for the two studies that had more than five distinct populations.

\section{Global meta-analysis}

We were interested in the magnitude, rather than the direction of, trait differences across populations and among genotypes; therefore, we analyzed the absolute values of LRRs. We used an 'analyze and transform' approach derived from Morrissey (2016) to account for the folded-normal distribution of absolute value effect sizes (Hereford et al. 2004; Kingsolver et al. 2012). First, we implemented Bayesian meta-analytic random-effects models in R (R Core Team, 2016) with the MCMCglmm package (Hadfield, 2010). We ran a separate model for each trait with study type as a fixed effect to obtain separate effect sizes for distinct population and genotype studies. Each trait may have a different level of sample error due to measurement techniques and technology. We account for measurement error in our analyses in two ways. First, we weighted the effect sizes by the inverse of the sample variance. Second, we have modelled each trait separately, allowing each trait to have a different error structure. The model structure was:

$$
\begin{gathered}
\hat{y}_{i j} \sim \beta * X+\text { study }_{i}+c g_{j \mid i}+m_{i j}+e_{i j} \\
m_{i j} \sim N\left(0, \boldsymbol{M}_{\boldsymbol{i j}}\right)
\end{gathered}
$$

where $y_{i j}$ is the untransformed LRR for the trait from the $j$-th common garden $(c g)$ which is nested in the $i$-th study; $\beta$ is the estimate of the fixed effect; $X$ is a dummycoded variable representing whether the LRRs were comparing distinct populations or genotypes; study ${ }_{i}$ represents the random intercept in the $i$-th study, $c g_{j / i}$ represents the random intercept for the $j$-th common garden in the $i$-th study, and $e_{i j}$ represents residual error. Effect size estimates were weighted using $m_{i j}$, which accounts for the known sample variance associated with trait $y_{i j}$ and the covariance structure of effect sizes through the use of matrices $\boldsymbol{M}_{\boldsymbol{i j}}$. For each common garden $\boldsymbol{j}$, the $\boldsymbol{M}_{\boldsymbol{i j}}$ matrix was constructed following Noble et al. (2017) to 
account for within-common-garden covariance of effect sizes due to pairwise comparisons (i.e., covariation because each population was represented in multiple contrasts). The matrix was created using the function make_VCV_matrix() (Noble, 2019). Every effect is represented by both a row and column in the matrix which has sample error variance on the diagonal and estimates of covariance between effect sizes on the off-diagonals. Covariance was estimated as $\operatorname{cov}(k, l)=$ $\mathrm{r} * \sqrt{V_{k}} \sqrt{V_{l}}$, where $k$ and $I$ are the row and column ID of the within-common-garden matrix, $V$ is the sampling variance calculated for each effect ("variance LRR" in equation 2) and $r$ is the correlation between effect size $k$ and I which was assumed to be 0.5 for all off-diagonal elements.

For each model, we used non-informative priors for fixed effects and inverseWishart priors for random effects (Hadfield, 2010). We ran the model for 150,000 iterations, discarded the first 30,000 as burn-in and used a thinning interval of 15 . We examined plots of Markov chain Monte Carlo to ensure good mixing. We generated posterior predictive distributions for each type of contrast (distinct populations or genotype comparisons) for each trait. These distributions were then transformed using a folded-normal distribution. This transformation allowed us to present the mode and 95\% highest posterior density intervals (95\% credible intervals) that relate to absolute effect sizes (Morrissey, 2016). When using absolute effect sizes, confidence intervals that do not cross zero are not necessarily indicative of effects that are significantly different than zero (Morrissey, 2016). Thus, we use the absolute effect size magnitude to compare each contrast of our study on the same scale.

\section{Effect of phosphorus environment on population traits}

We performed an analysis on a subset of data to investigate if populations from stoichiometrically distinct environments displayed a greater amount of trait variation than populations that were not identified as stoichiometrically distinct. For our stoichiometrically distinct populations we specifically looked at studies in which it was stated that resource $P$ availability differed between the environments from which the populations originated. We focused on environmental P availability in this subanalysis because $P$ is an essential, non-substitutable elemental resource for all life forms, and because it was the only element for which there were sufficient studies and observations to enable the analysis. Populations were considered to be from environments with similar $P$ availability if there was no mention of nutrient resource stoichiometry in the methods or results. After filtering studies, we had limited data for this analysis, therefore we focused on the three traits which had at least 10 pairwise comparisons: P content, AAA and somatic growth rate. We used the same statistical procedure as in the global meta-analysis 
to determine mean effect size, now using differences in $\mathrm{P}$ availability (binary Yes/No) as a modifier.

\section{Results}

From the 30 studies we found, 17 compared populations, 12 compared genotypes and one had both population and genotype comparisons (Table 1). The 18 studies comparing distinct populations had a total of 44 common gardens, with a range of 1-6 common gardens per study. Most of these studies were on invertebrates $(n=11)$ while comparatively fewer focused on vertebrates $(n=6)$ and plants $(n=1)$. The studies were overwhelmingly conducted on freshwater organisms $(n=15)$, with fewer terrestrial examples $(n=3)$. Eleven different genera were represented, with Daphnia being the most commonly studied $(n=5)$. Elemental composition was the ES trait measured most frequently (study $n=15$ ) while excretion $(n=5)$ and AAA traits $(n=8)$ were less commonly measured.

The 13 studies that assessed variation among genotypes within a population had a total of 23 common gardens, with a range of 1-4 common gardens per study. Most of the genotype studies were focused on plants (terrestrial $n=8$, marine $n=1$ ), and a few focused on freshwater invertebrates $(n=3)$ and vertebrates $(n=1)$. There were 8 genera represented, with the most common being Populus $(n=4)$ and Daphnia $(n=2)$. Similar to the population studies, the most frequently measured ES traits were elemental composition $(n=12)$ while excretion and AAA traits were each only measured in three studies.

Among the 30 studies included in our meta-analysis, 21 were based on populations of organisms that were obtained from ecosystems in North America. Of the nine remaining studies, eight were based on populations from northwestern continental Europe, and one was based on a single species of freshwater snail from New Zealand. We found no suitable studies of tropical organisms to include in our meta-analyses.

How much intraspecific genetic variation is present in ES traits relative to life history traits?

The magnitude of genetic variation was large for both life history traits, with effect sizes of $30.9 \%$ and $84.4 \%$ for morphology and somatic growth rate across populations, and of $32.8 \%$ and $146 \%$ for the same among genotypes. Most ES traits showed a moderate or small amount of genetic variation (i.e., absolute effect size; Figure 1) relative to life history traits. Differences among genotypes in AAA traits was the only effect size that was larger than life history traits (with an effect size of $164 \%$ ) and the magnitude of this effect can be partially attributed to a single 
study that found substantial genotypic effects, particularly for P retention (Sherman et al. 2017).

\section{Do particular classes of ES traits show more trait variation than others?}

Of the stoichiometric traits, excretion and AAA traits showed the most variation across populations and among genotypes, while elemental composition varied less (Figure 1). Contents of $\mathrm{P}, \mathrm{N}$ and $\mathrm{C}$ showed decreasing levels of trait variation (Figure 1), with particularly low levels of variation across populations and among genotypes in both $\mathrm{N}$ and $\mathrm{C}$. Consequently, nutrient ratios that include $\mathrm{P}$ show more trait variation than $\mathrm{C}: \mathrm{N}$ (Figure 1).

Is the magnitude of intraspecific genetic variation similar among genotypes (i.e., within a population) to what is observed across populations?

We found a similar magnitude of trait variation among genotypes that came from the same population as we did when we compared distinct populations (Figure 1). For some traits, namely AAA, $\mathrm{P}$ content, and $\mathrm{N}$ content we saw more trait variation among genotypes than among populations (Table 2). To ensure that this result was not an artifact of statistical methodology we conducted a supplemental analysis. We calculated LRRs for genotype studies using a different, more conservative approach and still found that there was similar or greater variation in traits when comparing genotypes than when comparing populations (Supplementary Material, Figure S1).

Do populations from different biogeochemical environments show more trait differentiation?

Our subanalysis included a total of 17 studies; two studies that differed in resource availability, but not specifically $P$, were removed from this analysis. Seven studies (16 common gardens) used populations originating from environments that differed in $\mathrm{P}$ availability. All seven studies focused on freshwater invertebrates, and five used Daphnia spp. Populations that were from environments with different $P$ availability did not show any more trait differentiation than those from populations without any known or measured biochemical differences (Figure 2). Somatic growth rate and $\mathrm{P}$ content showed nearly identical differentiation across populations that did and did not come from different $P$ environments (somatic growth rate: $85 \%$ and $100 \%$ difference, $\mathrm{P}$ content: $9.4 \%$ and $4.8 \%$ difference), while AAA traits varied more in populations that were from similar environments (Table 2; Figure 2). 


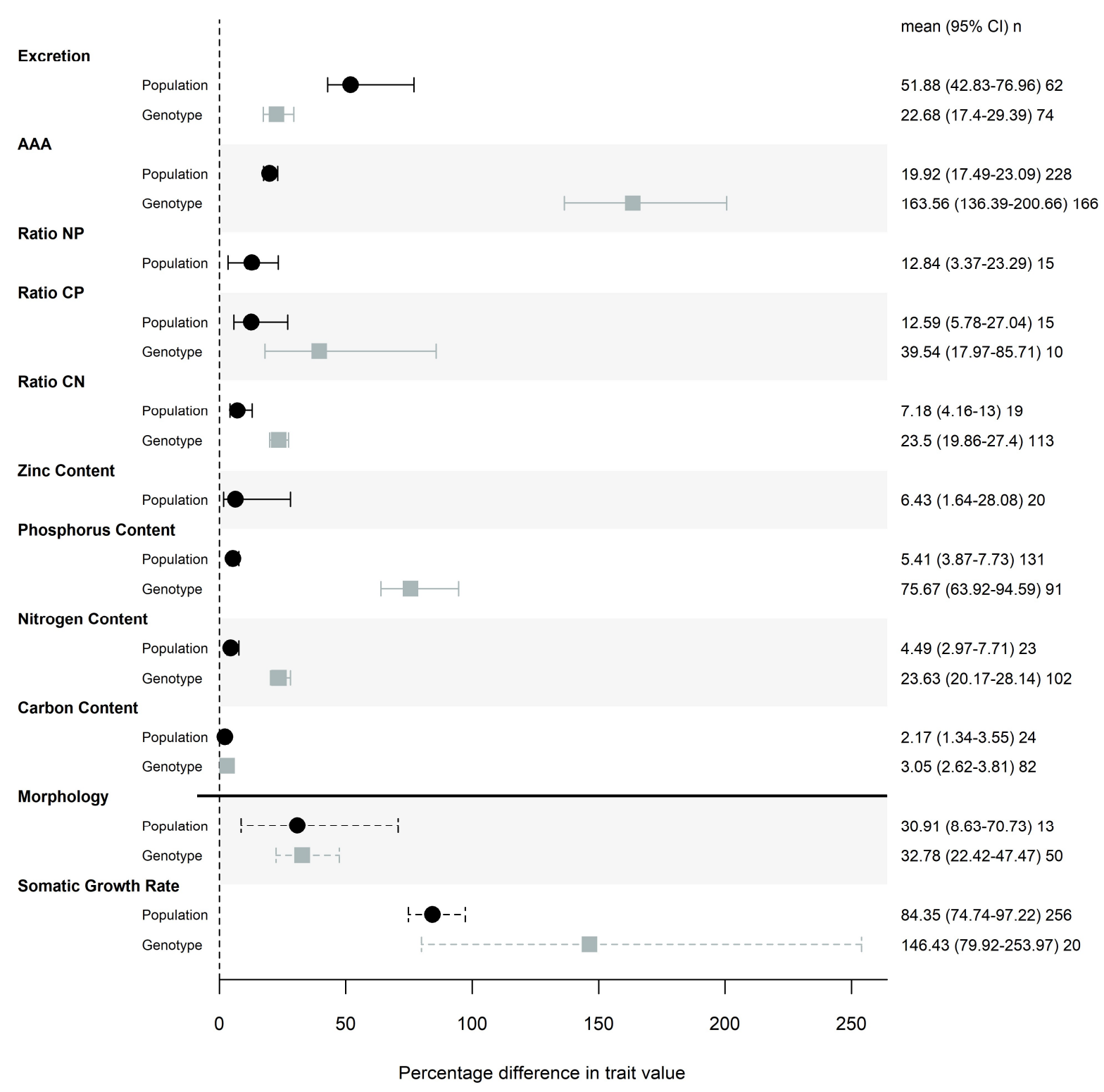

Figure 1. Magnitude of trait differences among pairs of populations (black) or genotypes (grey) grown in the same common garden. Points represent the mode of the posterior distribution and error bars are $95 \%$ credible intervals of the effect sizes. Posterior distributions of effects were taken from models that included common garden nested in study as a random effect, and the posterior distributions were transformed with a folded normal distribution to obtain the magnitude of difference between pairs of populations or genotypes. Percentage difference in trait values were calculated by back-transforming the mean effect sizes. $n$ represents the number of pairwise comparisons used to calculate each effect size. Somatic growth rate and morphology (measures of mass and size) effect sizes are denoted with a dashed line to indicate that this meta-analysis is not a comprehensive review of common garden experiments that measured these traits. 
Table 2. Deviance information criteria (DIC) of models for each trait with and without study type (global analysis) or stoichiometric environment (sub analysis) as a categorical variable. Positive $\triangle \mathrm{DIC}$ values indicate that the model with study type or stoichiometric environment as a fixed effect is the model that would best replicate a similar dataset. $\triangle D I C<2$ indicates little support for the inclusion of the categorical predictor variable, $2<\Delta D I C<10$ indicate some support and $\triangle D I C>10$ indicates strong support (Burnham et al. 2002). 'SGR' stands for Somatic Growth Rate.

\begin{tabular}{lllc}
\hline Analysis & Group & Trait & $\Delta$ DIC \\
\hline \hline Global & ES & Excretion & 13.08 \\
& & AAA & 398.55 \\
& & Ratio NP & NA \\
& & Ratio CP & 7.44 \\
& Ratio CN & 24.15 \\
& & Zinc content & NA \\
& & Phosphorus content & 404.94 \\
& & Nitrogen content & 44.63 \\
& & Carbon content & 10.61 \\
& \multirow{4}{*}{ Life History } & Morph & 3.15 \\
& & SGR & -0.28 \\
\hline Phosphorus & ES & AAA & 7.56 \\
environment & & Phosphorus content & 0.18 \\
sub-analysis & \multirow{2}{*}{ Life History } & SGR & -0.06 \\
\hline
\end{tabular}

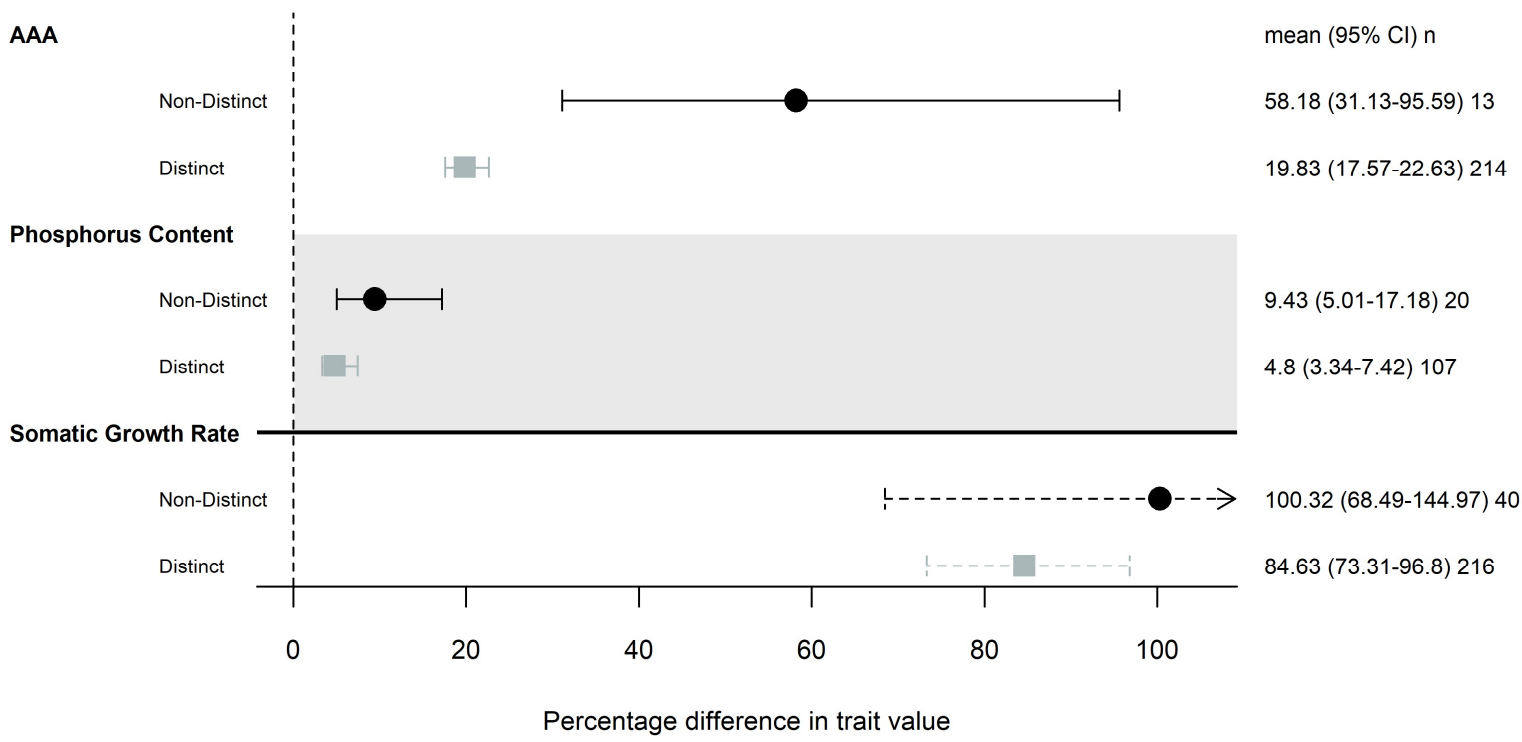

Figure 2. Magnitude of trait differences among pairs of populations that were from environments with distinct levels of $\mathrm{P}$ availability (grey) or not (black), that were grown in the same common garden. Only traits that had 10 pairwise comparisons were included in this analysis: somatic growth rate, acquisition, assimilation, and allocation (AAA) and phosphorus content. Points, error bars and line types as in Figure 1. 


\section{Discussion}

Our meta-analysis of common garden studies uncovered substantial intraspecific genetic variation in most ES traits. However, the magnitude of genetic variation that we observed for ES traits was generally less than what was observed for key life history traits measured in these same studies. Among ES traits, acquisition, assimilation, allocation, and excretion traits tended to vary more than elemental composition traits (i.e., the elemental content and stoichiometric ratios of organism biomass), and there tended to be markedly more variation among genotypes than across populations. Taken together, these results suggest that genetic variation in ES traits, particularly AAAE traits, within and across populations may be important for ecological and evolutionary outcomes.

\section{Evolution and the magnitude of genetic variation in ES and life history traits}

Our meta-analysis of common garden studies uncovered substantial genetic intraspecific variation in ES traits across a range of taxa. These findings join a growing body of literature that has demonstrated that variation below the species level can be both substantial and biologically important (Post et al. 2008; Schweitzer et al. 2004; Violle et al. 2012; Whitham et al. 2006, Crutsinger et al. 2006, Des Roches et al. 2018). In the light of these findings it is increasingly clear that incorporating data on intraspecific variation is likely to increase understanding of ecological patterns in nature (Bolnick et al. 2011). Our comparison of the amount of genetic variation in ES traits and the genetic variation in life history traits demonstrated that nearly all types of ES traits showed lower genetic intraspecific variation than life history traits. One clear exception was genetic variation among genotypes in AAA traits, which was influenced by a large amount of variation in one study (Sherman et al. 2017). If this difference in variation between ES and life history traits is genuine, it could be inferred that less genetic variation in ES traits is maintained within populations and that ES trait means evolve slowly across populations, in comparison to life history traits. It is certainly plausible that life history traits should vary more widely than ES traits, because constraints on organism elemental composition may be strong relative to those on some life history traits, and because trade-offs in element allocation within an organism could decouple variation in the total elemental content of an organism from variation in organism morphology and/or growth rate (Meunier et al. 2017).

The evolution of ES traits has been explicitly assessed in various ways in a small number of prior studies. Two studies have calculated the heritability of ES traits using genotypes from wild populations $\mathrm{H} 2=0.61$ Barbour et al. 2015; $\mathrm{H}^{2}=0.18$ 0.21 , Crutsinger et al. 2014a) suggesting that ES traits can respond to selection when genetic variation is present (but see Hansen et al. 2011). Indeed, artificial selection experiments have shown that all livestock species appear to have genetic 
variation for feed efficiency and this variation often has moderate heritability (Arthur \& Herd, 2005). Moreover, artificial selection experiments on ES traits tend to achieve rapid ES trait evolution in agricultural species (de Verdal et al. 2013; Mignon-Grasteau et al. 2017; Neely et al. 2008). Selection on life history traits can also lead to evolution in ES traits, for example, artificial selection on body size can produce rapid evolution in $\mathrm{P}$ content (Gorokhova et al. 2002). We were able to locate two selection experiments where environmental conditions were manipulated and evolution of ES traits were tracked through time in laboratory conditions. In one of these experiments ES traits did not evolve over 130 generations (Declerck et al. 2015) while the second experiment, which found that $\mathrm{N}$ and $\mathrm{P}$ content evolved in carbon-limited conditions, assessed trait evolution over 50,000 generations (Turner et al. 2017). We were only able to find a single instance of tracking the evolution of ES traits of natural or naturalistic populations (Frisch et al. 2014). In this study, the comparison of genotypes hatched from Daphnia resting eggs originating from a 700-year time interval in a single lake undergoing cultural eutrophication revealed an effect of $\mathrm{P}$ availability on AAA traits but not elemental composition (Frisch et al. 2014). Clearly, much more work is needed to determine whether and when ES traits evolve rapidly in natural contexts. Selection experiments conducted in nature or observational studies that track the evolution of populations through time in both ES and life history traits would provide valuable empirical data on the rate of evolution of ES phenotypes with realistic population dynamics and selective landscapes. These types of approaches could improve the understanding of whether and why ES traits tend to show less genetic variation than life history traits.

\section{Differences in the magnitude of genetic variation among stoichiometric} traits

One of the fundamental principles of ES theory is that the elemental composition of organisms is constrained by their basic requirements for the non-substitutable elemental resources that are allocated to fundamental biological structures and processes (Reiners, 1986; Sterner \& Elser, 2002). Many of these life-enabling structures and processes are ancient (e.g., bone and wood; Morris et al. 2018; Wagner \& Aspenberg, 2011), and some are as old as life itself (e.g., RNA synthesis; Joyce, 1989). Thus, it is perhaps not surprising that we found little genetic variation in bulk elemental composition within species, because any contemporary variation is miniscule relative to that found over the entirety of evolutionary history. In other words, the magnitude of rapid evolution in elemental composition is limited relative to what is seen across species with deep evolutionary divergence.

However, there was substantially more intraspecific genetic variation present in the AAAE traits than in the elemental composition traits, and this was the case for both 
genotype and population comparisons (Figure 1). This leads to questions about constraint of genetic diversity for elemental composition relative to AAAE traits. Acquisition, assimilation, allocation, and excretion are traits which actually contribute to and maintain elemental composition within the necessary range for organismal growth, maintenance, and survival (Sterner \& Elser, 2002). Thus, our results suggest that there is more genetic variability in the strength of stoichiometric homeostasis than there is in the actual bulk elemental composition of organisms. This makes sense, given that genetic variation in AAAE traits is a prerequisite for genetic variation in elemental composition (Jeyasingh et al. 2014). Indeed, elemental composition reflects the 'balance' between the acquisition, assimilation, allocation, and excretion of elements at a given point during an organism's life. An evolved change in elemental composition would, therefore, presumably entail a corresponding heritable shift in the balance among AAAE traits. Importantly, this does not mean to say that elemental composition does not evolve. Over macroevolutionary timescales evolution has generated an enormous diversity of stoichiometric phenotypes across taxa (Sterner \& Elser, 2002). Yet much of this stoichiometric diversity has emerged not necessarily because ES traits are (or were) under direct selection, but because they are mechanistically correlated with functional traits that have had direct fitness implications. For example, the high C:nutrient ratio of a woody tree is a consequence of selection for height, which imparts an obvious competitive advantage to terrestrial autotrophs. Wood provides the rigid structural material necessary for this height and has a C:N ratio 200-1000 (Levi \& Cowling, 1969). Thus, as entire organisms, trees have high $\mathrm{C}: \mathrm{N}$ ratios relative to other organisms, but not because $\mathrm{C}: \mathrm{N}$ ratio was under direct selection per se. Another example of indirect selection on ES traits with clearer implications for rapid evolution, comes from the stoichiometric growth rate hypothesis (GRH), which asserts that the functional trait of rapid growth has a $\mathrm{P}$ rich signature due to the high $\mathrm{P}$ content of ribosomal RNA (Elser et al. 2000c; Sterner \& Elser, 2002). Identifying the stoichiometric patterns associated with variation in a larger suite of functional traits will be tremendously valuable to the study of evolutionary and ecological relationships (Meunier et al. 2017).

Within the elemental composition traits, we found more variation in $\mathrm{P}$ content than in $\mathrm{C}$ and $\mathrm{N}$ content. This is consistent with the argument that variation in organism $\mathrm{P}$ content will generally be greater than variation in organism $\mathrm{C}$ and $\mathrm{N}$ content, because levels of $\mathrm{C}$ and $\mathrm{N}$ tend to be similar among major biomolecules (i.e., protein and nucleic acids) while P content varies widely among biomolecules (e.g., protein contains no P, while RNA contains around 9.2\% P; Sterner \& Elser, 2002). Thus, changes in proportional concentrations of major biomolecules on a cellular level can scale up to drive variation in overall organism $\mathrm{P}$ content. Our results 
provide a novel genetic perspective that supports the view that $\mathrm{P}$ content is more variable than $\mathrm{C}$ and $\mathrm{N}$, at least in the case of genotype comparisons.

\section{Genetic variation in ES traits within populations and across population pairs}

We collected data on the amount of genetic variation by comparing across population pairs and among genotypes from the same population. The extent of variation among genotypes was generally larger than variation in population trait means, even when using a conservative methodology to calculate genotypic variation (Supplementary Material, Figure S1). When comparing across populations, we assessed shifts in the means between pairs of populations, which does not explicitly account for variation within populations. As such, our finding that genetic variation is generally greater amongst genotypes within a population than across populations reflects that the average genetic trait difference between genotypes is larger than the average trait shift between populations. Finding lower levels of variation across populations than among genotypes indicates that there is either little differentiation across populations, or large amounts of variation among genotypes. Whether this is to be expected or not depends on where genetic variation within a species is expected to be found: within or across populations. Genomic sequencing both within and across populations has uncovered support for each, including cases where the majority of genetic variation is found within populations (Pometti et al. 2015) and cases where the majority is found among them (Bakker et al. 2006). From the latter perspective this result is surprising, given that genetic variation within a population is limited by recombination amongst genotypes, which would serve to limit divergence within a population (Ehrlich \& Raven, 1969; Jain \& Bradshaw, 1966). There is potential for increased variation across populations because they originated from different environments, which have the potential to act as an agent of divergent selection driving local adaptation, whereas the genotypes compared mostly originated from the same environment. Genotypes may show a high amount of variation due to fluctuations in the environment, gene flow, or any other mechanism that can enhance the maintenance of trait diversity. On the other hand, trait divergence between populations may be limited if they do not come from stoichiometrically-disparate environments (e.g., little divergent selection on ES traits) or do not show substantial local adaptation due to stoichiometric constraints.

Our data provide some ability to assess the role of environmental differences, specifically $P$ availability, in driving divergence in ES traits across populations. Populations that come from divergent $P$ environments and those that come from similar $\mathrm{P}$ environments showed no differences in the amount of genetic variation in ES traits. This suggests that natural environmental differences in elemental availability may not be a sufficiently strong agent of selection to drive rapid 
evolution in ES traits, which could explain the largely similar levels of variation observed across genotypes and pairs of populations. Determining whether the limited variation we observed across natural populations is due to comparatively modest environmental stoichiometric differences or other factors that could limit divergence of populations (e.g., low divergence times, connectance between populations, reduced efficacy of selection) would be valuable in addressing this disparity.

\section{Caveats and limitations of the meta-analysis}

There are several limitations in our analysis stemming from the meta-analysis framework, the nature of common gardens, and the specific designs of the studies available. First, studies that met the criteria for our meta-analysis included both plants and animals, but those that measured variation across genotypes were dominated by plants and cladocerans. Thus, inherent differences among taxonomic groups may be confounded with the genotype- and population-level comparisons, though the relative amounts of variation were similar among all taxonomic groups. Second, genotype-level variation may be overestimated relative to natural populations, because some studies included genotypes with low ecological fitness (e.g., Sherman et al. 2017) and some collected genotypes from wide geographical areas that covered a range of environments similar to the magnitude of variation we would expect across populations. Third, many of the common gardens were only conducted for a single generation, meaning that maternal and epigenetic effects could represent latent sources of trait variation (Weaver et al. 2004). Finally, a 'common garden' allows for measurement of the amount of genetic variation in traits in a single environment and the chosen environmental conditions may influence the amount of trait variation displayed. For example, there was more variation in P-acquisition and assimilation under P-limited conditions relative to P-sufficient conditions (Sherman et al. 2017). However, we assumed that the common garden conditions were chosen by the original study authors to reflect ecologically-relevant environments, and that the trait variation we observed is, therefore, a reasonable representation of that found in nature.

\section{Ecological consequences of intraspecific variation in ES traits and the potential for eco-evolutionary feedbacks}

Over the past two decades there has been a growing recognition that intraspecific trait variation can have large effects on ecological patterns and processes (Bassar et al. 2010; Crutsinger et al. 2006; Des Roches et al. 2018; Harmon et al. 2009; Post et al. 2008; Schweitzer et al. 2004; Violle et al. 2012; Whitham et al. 2006). Intraspecific variation in ES traits has been identified as particularly likely to have strong ecological consequences because ES traits connect the organism with its 
environment. Our meta-analysis uncovered substantial differences in the amount of intraspecific variation across types of ES traits, with excretion and AAA traits showing considerably more intraspecific variation than most elemental ratios and measures of elemental composition. Although there have been few empirical tests of the ecological consequences of intraspecific variation in ES traits (but see Chowdhury \& Jeyasingh, 2016), any evolution that shapes these traits is likely to have ecological consequences (El-Sabaawi et al. 2016; Jeyasingh et al. 2014). Future experimental work that measures the ecological consequences of genetic variation in ES traits in ecologically realistic contexts will be crucial to determining the importance of intraspecific variation in ES traits for community structure and ecosystem function.

Ideally, this future work will indicate whether rapid evolution of ES traits is likely to have ecological consequences, and whether these ecological consequences could lead to rapid evolution (i.e., an eco-evolutionary feedback). Using a strict definition of eco-evolutionary feedback (Schoener, 2011), two interacting processes would be required: ES traits would have to evolve rapidly in a population and the evolution of these ES traits would need to modify the environment in a way that significantly affects selection on that population. The potential for eco-evolutionary feedbacks mediated by the evolution of ES traits has been much discussed (Jeyasingh et al. 2014; Leal et al. 2017b; Rudman et al. 2019), and some studies have demonstrated that rapid evolution can shape elemental availability (Bassar et al. 2010; Rudman \& Schluter, 2016). Yet, whether these differences in elemental availability feed back onto rapid evolution is wholly unknown. As such, determining the existence, magnitude, and prevalence of these eco-evolutionary feedbacks is well beyond the scope of our meta-analysis, especially as there is very little empirical evidence of eco-evolutionary feedbacks overall (Matthews et al. 2016; Rudman et al. 2018; Schoener, 2011; Turcotte et al. 2011). What little data our meta-analysis does provide to examine eco-evolutionary feedbacks based on ecological stoichiometry does not support widespread rapid evolution of ES traits, as variation across populations was similar to what was observed amongst genotypes found within populations. Moreover, we did not find evidence that divergence in $\mathrm{P}$ environment led to enhanced divergence in ES traits across populations, suggesting that environmental variation might need to be substantial to drive ES trait evolution. Nevertheless, our findings are mere hints at the prevalence of eco-evolutionary feedbacks mediated by ES traits and future work is certainly warranted. 


\section{Acknowledgements}

We would like to thank Jim Elser, Michelle Evans-White and Joe Vanderwall for their efforts that made the Woodstoich IV workshop possible. We thank our Woodstoich IV mentors, Jotaro Urabe and Jennifer Schweitzer for feedback and helpful discussions. We thank Daniel Noble and Vincent Fugère for valuable guidance on the statistical analysis. 


\section{Supplementary Materials}

\section{Appendix S1: Additional Methodological Information}

Sensitivity of effect size calculations for comparing genotypes

To ensure that our method for calculating effect sizes for genotype studies was not contributing to the large amount of genotypic variation we observed in the data, we conducted a secondary, more conservative calculation for the log response ratios (LRRs) using the following formulae:

$$
\begin{gathered}
L R R_{\text {median }}=\ln \left(\frac{{\overline{\text { Tralt }_{1}}}_{\text {Tralt }_{\text {median }}}}{\text { Trian }}=\frac{S D_{1}{ }^{2}}{n_{1}\left(\text { Tralt }_{1}\right)^{2}}+\frac{S D_{\text {median }}{ }^{2}}{n_{\text {median }}\left(\text { Tralt }_{\text {median }}\right)^{2}}\right. \\
\text { variance } L R R_{\text {median }}
\end{gathered}
$$

We calculated all LRRs using equation S1 with the median trait value as the denominator. This was conservative because the LRRs produced are a subset of all pairwise comparisons that does not include LRRs that compare genotypes from either end of the trait distribution. Genotypic variation was generally higher than the variation found across populations (Figure S1). 




\section{Chapter 3}

An experimental test of the Growth Rate Hypothesis as a predictive framework for microevolutionary adaptation

Kimberley D. Lemmen, Libin Zhou, Spiros Papakostas, \& Steven A.J. Declerck 


\section{Abstract}

The growth rate hypothesis (GRH), a central concept of ecological stoichiometry, posits that organismal somatic phosphorus content $\left(\mathrm{P}_{\text {som }}\right)$ is positively related to somatic growth rate, and has strong support at the interspecific level. Here, we explore the intraspecific applicability of the $\mathrm{GRH}$, and thus its potential to predict microevolutionary responses. For this, we subjected zooplankton populations to selection for fast population growth (PGR) in P-rich (HP) and P-poor (LP) food environments. With common garden transplant experiments we demonstrate that in HP populations evolution towards increased PGR was concomitant with an increase in $P_{\text {som. In }}$ contrast, LP populations evolved higher PGR without an increase in $\mathrm{P}_{\text {som. }}$. We conclude that the $\mathrm{GRH}$ has the potential to predict microevolutionary change, but that its application is contingent on the environmental context. Our results highlight the potential of cryptic evolution in determining the performance response of populations to elemental limitation of their food resources.

\section{Keywords}

ecological stoichiometry, phosphorus limitation, intraspecific genetic variation, experimental evolution, rapid adaptation, contemporary evolution, zooplankton, rotifera, Brachionus calyciflorus 


\section{Introduction}

Microevolutionary responses to environmental change frequently occur on ecologically-relevant timescales (Hairston et al. 2005; Carroll et al. 2007), and have the potential to be predictable at the phenotypic level (Lässig et al. 2017). Given the unprecedented rate and magnitude of contemporary environmental change (Elser et al. 2009; Collins et al. 2013), understanding the trajectory of adaptive responses within populations becomes increasingly relevant. Ecological stoichiometry has utilized the flow of elements and their relative abundances to better understand ecological patterns and processes (Sterner \& Elser 2002). This framework could also be useful to anticipate evolutionary responses as the quantity and ratios of elements can act both as a selective pressure (e.g., resource quality) or as features that respond to selection (e.g., elemental composition) (Matthews et al. 2011; Leal et al. 2017b). Considering selective forces in a stoichiometric context may therefore allow us to forecast evolutionary trajectories and their ecological impacts.

A key concept in ecological stoichiometry is the growth rate hypothesis (GRH). The $\mathrm{GRH}$ posits that organismal phosphorus $(\mathrm{P})$ content is positively related to somatic growth rate (SGR) (Figure 1), because growth requires protein synthesis, which depends on Prich ribosomes (Elser et al. 1996). As nucleic acids represent a large proportion of somatic $P$ in smallbodied invertebrates (Vrede et al. 1999; Gillooly et al. 2005), changes in the abundance of ribosomal RNA (rRNA), a primary component of ribosomes, can affect whole-organism stoichiometry. Thus, the GRH ascribes differences in organismal $\mathrm{C}: \mathrm{N}: \mathrm{P}$ to differential investment in protein synthesis (Sterner \& Elser 2002). As such, faster-growing organisms are predicted to have higher P-content than slower growing organisms. Multiple studies provide empirical support for the GRH via correlations between SGR and RNA/P-content

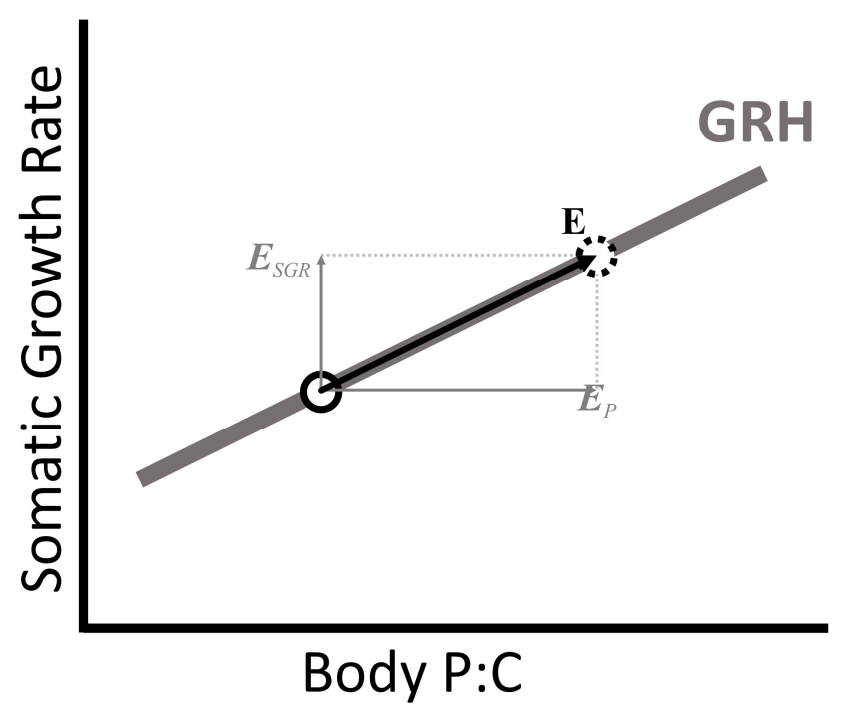

Figure 1. The growth rate hypothesis (GRH) predicts a positive association between somatic $\mathrm{P}$-content and somatic growth rate (solid line). If the GRH is applicable at the intraspecific level, directional selection for higher somatic growth rates are expected to result in increased somatic P-content. The vector $E$ represents the evolutionary trajectory of a population predicted by the $\mathrm{GRH}$, and the respective increases in somatic growth rate $\left(E_{S G R}\right)$ and P-content (EP). The ancestral and evolved populations are represented by circles with solid and dashed outlines, respectively. 
across taxa (Elser et al. 1996, 2000c, 2003; Ferrão-Filho et al. 2007; Mouginot et al. 2014).

Although currently unresolved at the intraspecific level, the GRH holds great potential as a framework for predicting microevolutionary change in populations. For such an approach to be applicable, organismal P-content and somatic growth rate both need to be heritable and show a strong genetic correlation. Currently there is insufficient evidence of such correlation. Studies conducted with single genotypes (e.g., DeMott et al. 1998; Acharya et al. 2004; Kyle et al. 2006), provide support for a physiological, but not a genetic association between $\mathrm{P}$-content and growth. Moreover, studies using multiple genotypes rarely quantify the genetic component of the response (e.g., Fink \& Von Elert 2006; González et al. 2014; Prater et al. 2018). The limited number of studies that do allow for a direct test of $\mathrm{GRH}$ predictions at the intraspecific genetic level have reported evidence for considerable heritable variation in SGR but less so for P-content, and were inconclusive regarding the genetic relationship between both variables (Arnold et al. 2004; Weider et al. 2004; Liess et al. 2013; Sherman et al. 2017). Thus, evidence for the GRH at the intraspecific level is currently inconclusive and its predictive power is untested. At the intraspecific genetic level, a lack of a positive association between P-content and SGR could be explained by any (combination) of the following: (1) SGR is predominantly determined by growth efficiency per unit of body $\mathrm{P},(2)$ physiological rates involving mass specific $P$-acquisition and retention are more important determinants of SGR than P-content itself (Sherman et al. 2017), and (3) genetic variation in SGR is controlled by traits unrelated to Pmetabolism. However, in cases where the GRH would hold at the intraspecific level, knowledge of intraspecific genetic variation for somatic P-content would allow predicting the capacity of populations to evolve faster growth rates. Alternatively, it could be used to predict changes in somatic $P$ in response to environmental contexts that select for fast population growth. Enhanced predictive power with regard to consumer body stoichiometry could in turn inform potential effects on ecological processes such as nutrient cycling (Elser \& Urabe 1999) and trophic dynamics (Hall et al. 2007; Boersma et al. 2008).

Here, we applied an experimental evolution approach to evaluate the power of the $\mathrm{GRH}$ to predict evolutionary responses of populations to selection for fast population growth rate (PGR). The GRH states that high body P-content enables organisms to grow faster. When applied in a microevolutionary context, and assuming that SGR and PGR are strongly associated (Lampert \& Trubetskova 1996; Zhou \& Declerck 2019), we expect that populations evolving in response to selection for fast population growth should become dominated by fast growing genotypes with relatively high somatic P-content (Gorokhova et al. 2002). 
However, if the GRH does not hold at the intraspecific level, populations may respond to selection for fast population growth without a simultaneous increase in P-content (e.g., through a more efficient P-metabolism or altered life history). As reliance on $\mathrm{P}$ should be especially maladaptive in a P-poor environment (Sterner \& Hessen 1994; Sterner \& Elser 2002; Seidendorf et al. 2010), we anticipate the power of the GRH to predict evolutionary responses will be influenced by Pavailability. To test these predictions, we subjected genetically diverse populations of the microzooplankton Brachionus calyciflorus to a culturing regime selecting for fast population growth using food with either a high or low P-content at satiating concentrations. We then evaluated evolutionary responses by rearing the evolved and ancestral populations in a common garden experiment to compare population-level traits associated with fitness and P-stoichiometry. Our study is unique in that it empirically tests the idea that evolution towards increased population growth rate will be accompanied by an increase in body P-content. As such, it allows us to explore the applicability of the GRH at the intraspecific level, and to test its ability to predict microevolutionary trajectories.

\section{Material and Methods}

Model organism

B. calyciflorus, is a cyclical parthenogenetic planktonic monogonont rotifer, capable of reproducing asexually and sexually. Asexual reproduction produces subitaneous eggs allowing for rapid clonal population growth. In contrast, sexual reproduction produces diapausing embryos in so-called 'resting eggs' (Stelzer 2017). The propensity for sex varies between genotypes (Becks \& Agrawal 2013), and high rates of sex typically result in reduced PGR (Serra \& Snell 2009; Stelzer 2011).

\section{Origin and maintenance of algal and rotifer cultures}

We used thirty distinct genotypes to initiate the evolution experiment (further referred to as 'seed' genotypes; Table S1). B. calyciflorus is part of a cryptic species complex which hitherto is comprised of four cryptic species (Michaloudi et al. 2018) that often hybridize (Papakostas et al. 2016). Our microsatellite analysis showed evidence of hybridization between the sister species $B$. calyciflorus and $B$. elevatus for seven of the 'seed' genotypes (Appendix S1). We included these genotypes to incorporate genetic diversity representative of many natural populations. All genotypes were maintained in asexually reproducing stock cultures with nutrient replete resources (Appendix S1). 
We used the motile green algae Chlamydomonas reinhardtii as a food resource in all experiments. To produce high ('HPF': molar C:P ratio $121 \pm 11.9 \mathrm{SE}$ ) and low phosphorus ('LPF': molar C:P $671 \pm 9.9 \mathrm{SE}$ ) algae we varied the P-content of the WC medium (Kilham et al. 1998) and light intensity (Appendix S1).

\section{Evolution Experiment}

To initiate the evolution experiment we assembled fourteen replicate populations with identical genetic composition by combining two females with a single asexual egg from each of the seed genotypes. We randomly allocated seven of the populations to a high (HPF) and the other seven populations to a low (LPF) phosphorus diet. All populations were cultured in $48 \mathrm{~mL}$ of the designated algal suspension at a concentration of $1550 \mu \mathrm{mol} \mathrm{L}{ }^{-1} \mathrm{C}$ and maintained in the dark at a constant temperature of $24 \pm 1^{\circ} \mathrm{C}$.

Every 24 hours we transferred 60 haphazardly selected individuals and all resting eggs from each population to a new culturing flask with a fresh food suspension. By transferring a subset of the populations daily, we selected for fast clonal population growth as genotypes that produced the most offspring were more likely to be transferred. Food concentrations were provided ad libitum, which prevented density regulation of PGR due to intraspecific exploitative competition (i.e., density dependence). By transferring diapausing eggs, we allowed for sexually recombinant genotypes to establish.

After the daily transfer, we counted the remaining individuals. We calculated PGR as $\left(\ln N_{t}-\ln N_{0}\right) / t$, where $N_{0}$ and $N_{t}$ represent the population size at the start and end of each 24-h period, and the duration of the period in days. The evolution experiment lasted 36 days. At the end of the experiment, we performed a microsatellite analysis to determine the genetic composition of each final population (Appendix S1). Following the conclusion of the experiment all populations were maintained in the culturing conditions of the evolution experiment for later use in common garden experiments.

\section{Common Garden One (CG1): PGR and Fraction of Sexual Individuals}

Using the evolved populations we performed a common garden experiment to test for genetic adaptation to selection for fast growth in the two food quality treatments. Due to logistical constraints, we randomly chose five of the seven evolved populations per selection treatment (Table S2). We cultured four technical replicates of each of these populations in each common garden environment (HPF or LPF). In addition, to estimate the ancestral state we cultured one population for 
each of ten randomly selected seed genotypes in each common garden environment.

We initiated each experimental unit with ten rotifers and provided $8 \mathrm{~mL}$ of algal suspension at a concentration of $1550 \mu \mathrm{mol} \mathrm{L}{ }^{-1} \mathrm{C}$. The first common garden lasted for 15 days. Every 24 hours we transferred ten haphazardly chosen individuals from each experimental unit into a fresh algal suspension. We counted the remaining animals to estimate PGR and preserved them in $4 \%$ formalin solution. Data from the first five days were omitted from the calculations to avoid maternal effects from previous culturing conditions (Zhou \& Declerck 2020). To determine the fraction of sexual females for each replicate, we examined all preserved individuals and determined the number and type of eggs they carried (Appendix S1). The fraction of sexual females was defined as the number of females with sexual eggs (male and diapausing eggs) divided by the total number of egg-bearing individuals (adults with male, diapausing, or amictic eggs).

\section{Common Garden Two (CG2): Rotifer Elemental Composition}

A second common garden experiment was performed to evaluate the effect of selection history on organismal carbon $(C)$, nitrogen $(N)$, and phosphorus $(P)$ content. The design of this experiment was similar to CG1. However, because the microsatellite analysis revealed some populations were dominated by the same genotype, we removed two populations from the experimental design to avoid redundancy (Table S2). Given that the quantification of rotifer elemental composition requires a large number of individuals, cultures in CG2 were upscaled in comparison to CG1 (Appendix S1).

\section{Life History Experiment}

As propensity for sex is known to strongly affect PGR (Stelzer 2011), we conducted an abbreviated life table to assess the proportion of sexual individuals in LP- and HP-evolved populations in LPF diet (see details in Appendix S1).

\section{Data Analysis}

To evaluate temporal trends in PGR during the evolution experiment we compared the fit of two alternative models, a piecewise and linear regression model (Appendix S2).

Microsatellite analysis revealed the existence of two very different groups of populations (Table S2): (i) populations composed of one of two of the original seed clones identified as hybrids (further referred to as "hybrid" populations) and (ii) populations composed of one or multiple unique multilocus genotypes 
produced during the evolution experiment via sexual recombination of the $B$. calyciflorus species ("non-hybrid" populations). Hybrids differed from non-hybrids in several important traits - PGR and sexual investment. As to not obscure population responses to the experimental treatments, we analyzed hybrid and non-hybrid populations separately.

We performed simulations to evaluate the probability that trait changes in the evolved populations resulted from selection rather than from drift. We refer to Appendix S2 for a detailed account of the simulation methodology. Briefly, we initiated neutral-evolution simulations for a trait by assigning to 30 genotypes trait values drawn from a normal distribution with the same mean and variance as measured for the ten seed genotypes in each of the food quality treatments. Following the design of the evolution experiment, these genotypes were used to create three identical replicate populations that were subjected to the same subsampling procedures as in the evolution and common garden experiments. All simulated genotypes were assigned the same PGR which was equal to the mean PGR of the seed genotypes during CG1 in the respective food quality treatments. We calculated trait means for the neutrally evolved populations based on genotype frequencies in the final populations. We then calculated the difference between mean traits of three simulated neutrally evolved populations and three values drawn from a normal distribution with the same mean and variance as measured for a given selection history in the common garden experiment. For each trait, this procedure was repeated 10,000 times. If $97.5 \%$ of the differences were either all larger or smaller than zero, then trait differentiation was considered greater than neutral expectations.

The effect of selection history (HP-vs. LP-selected non-hybrid populations) and its interaction with food quality (HPF vs. LPF) in the common garden experiments was tested using linear (LMM) or generalized linear mixed effect models (GLMM) depending on the error structure of the response variable. LMMs were used for PGR, elemental traits and PGR per unit body P, whereas GLMMs with binomial error and logit link were used to analyze the fraction of sexual individuals. For all response variables, population ID was used as a random factor to account for repeated measures. If the GLMM was overdispersed a replicate level random factor was included (Harrison 2014). Using the same model structure, a second set of analyses was performed on the same response variables to test the effect of genetic background (hybrids vs. non-hybrids) in interaction with common garden food quality. Due to the limited number of true replicates, the response of hybrid populations from HP and LP selection regimes were combined for a comparison with non-hybrid populations in their 'home' environment. 
All statistical analyses were performed in R software environment 3.6.1 (R Core Team, 2019). LMM and GLMM analyses were performed with the Ime4 package (Bates et al. 2015). Statistical significances were obtained from type II sums of squares using the car package (Fox \& Weisberg 2019), LMM used Kenward-Roger degrees of freedom. Post-hoc comparisons were performed with emmeans (Lenth 2019).

\section{Results}

\section{Evolution Experiment}

In contrast to LP-selected populations, HP-selected populations showed an increase in PGR throughout the evolution experiment $\left(R^{2}=0.20, F(1,76)=18.88\right.$, $\mathrm{p}<.001$, Table S3, Figure S1). In all populations, the production of resting eggs initially increased, peaking between day five and ten after which it declined (Figure S2). At the experiment's conclusion, genetic diversity had been reduced to a single multilocus genotype (MLG) in 12 of the 14 populations. The two other populations were dominated by four or more MLGs (Table S2). Eight of the final populations (4 HP, 4 LP), were entirely dominated by one of two seed clones identified as hybrids. The remaining six populations ( $3 \mathrm{HP}$, and $3 \mathrm{LP}$ ) were dominated by new sexually produced unique non-hybrid MLGs.

\section{Non-Hybrid Populations in Common Garden Experiments}

Population growth rate and population structure

In the HPF treatment, PGR of non-hybrid populations with an HP selection history was significantly higher than values simulated for neutrally evolved ancestral populations (Figure 2, Table S5). Similarly, in the LPF treatment, non-hybrid populations with an LP selection history were characterized by a significantly higher PGR than neutrally evolved ancestral populations (Figure 2, Table S5). The LPF treatment substantially reduced PGR in all populations. We also observed a significant interaction effect between former selection history and food quality (Figure 2, Table 1). In the HPF treatment we observed no effect of selection history on PGR. Conversely, in the LPF treatment, populations with a LP selection history had a greater PGR than populations with a HP selection history (Figure 2, Table 1).

Across common garden treatments we observed no differences in the proportion of females with sexual eggs between non-hybrid populations from the evolution experiment and the simulated values for neutrally evolved ancestral populations (Table S5, Figure S3). The interaction between food quality and selection history was significant for the fraction of sexual individuals (Table S6). However, the life history experiment in the LPF treatment revealed no differences in the propensity 


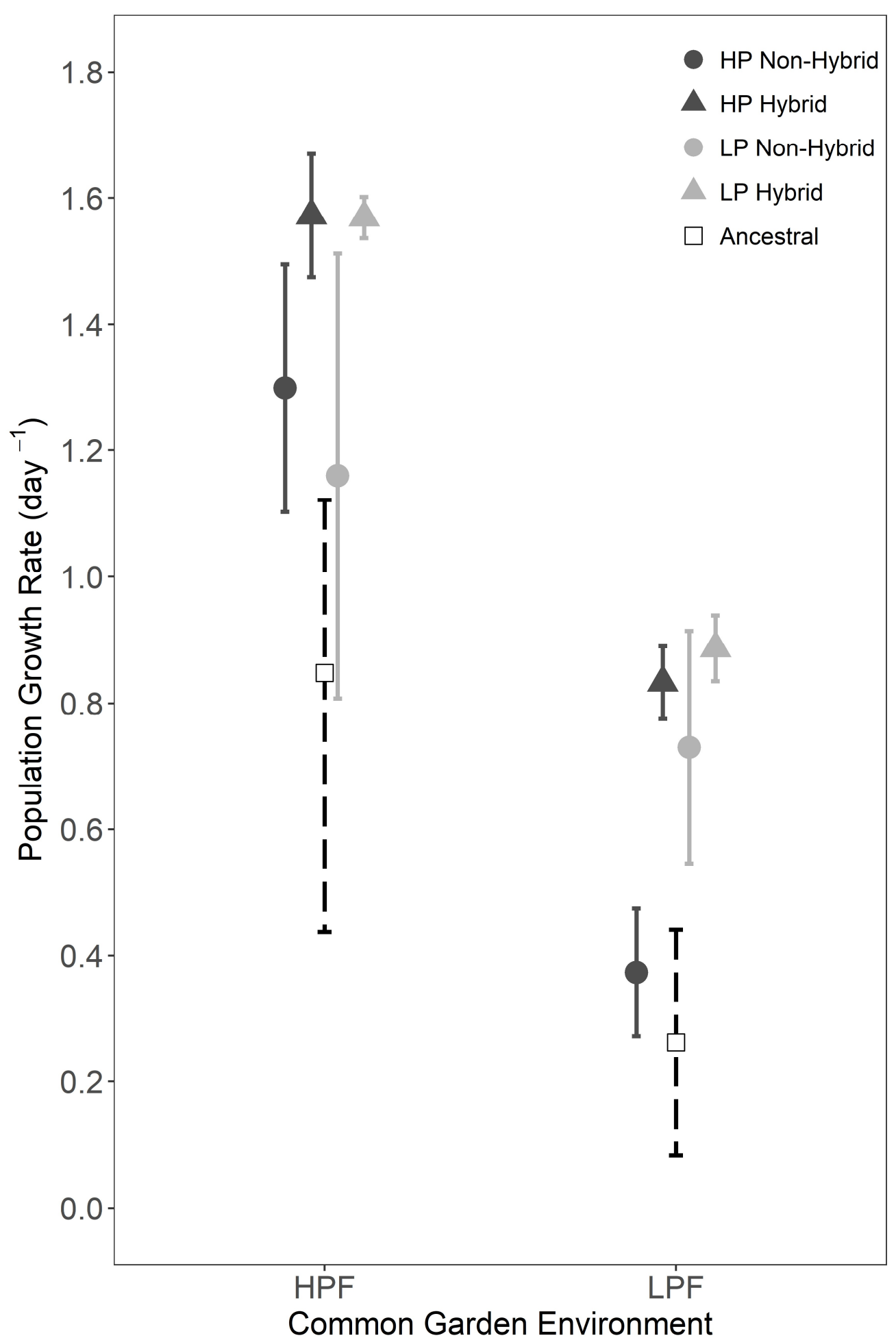

Figure 2. Population growth rate for evolved populations and the ancestral population in the first common garden experiment (CG1) with high (HPF) and low phosphorus diets (LPF). During the evolution experiment, populations evolved in either a high (HP) or low phosphorus (LP) selection regime and were composed of either non-hybrid or hybrid genotypes. For evolved populations we present means \pm 2 standard errors (solid line; non- hybrid, $n=3$; hybrid, $n=2$ ). The ancestral population means and $95 \%$ confidence intervals were obtained by bootstrapping the values observed for a subset of seed genotypes (dashed line; Table S2). 
for sex between populations from both selection histories and the simulated neutrally evolved ancestral populations (Table S5).

Table 1. Summary of linear mixed effects analyses for the population growth rate (see also Figure 2). Non-hybrid and hybrid populations were analyzed separately and the effects of diet (low or high phosphorus) and selection history (HP or LP evolved) are presented as the fixed components of the models.

\begin{tabular}{|c|c|c|c|c|c|c|}
\hline & Sum $\mathbf{S q}^{\mp}$ & Mean Sq ${ }^{\mp}$ & NumDF $^{\mp}$ & DenDF $^{\mp}$ & F value & $\mathbf{p}^{\mp}$ \\
\hline \multicolumn{7}{|l|}{ Non-Hybrid } \\
\hline Diet & 5.513 & 5.513 & 1 & 40 & 381.78 & $<0.001$ \\
\hline \multicolumn{7}{|l|}{ Selection } \\
\hline History & 0.007 & 0.007 & 1 & 4 & 0.51 & 0.516 \\
\hline Diet $\times$ SH & 0.736 & 0.736 & 1 & 40 & 50.95 & $<0.001$ \\
\hline \multicolumn{7}{|l|}{ Hybrid } \\
\hline Diet & 4.041 & 4.041 & 1 & 26 & 987.46 & $<0.001$ \\
\hline \multicolumn{7}{|l|}{ Selection } \\
\hline History & 0.005 & 0.005 & 1 & 2 & 1.19 & 0.389 \\
\hline Diet $\times \mathrm{SH}$ & 0.007 & 0.007 & 1 & 26 & 1.61 & 0.216 \\
\hline
\end{tabular}

\section{Rotifer elemental content and ratios}

Irrespective of selection history, non-hybrid populations from the evolution experiment had a significantly higher individual P-content in the HPF treatment than neutrally evolved ancestral populations (Figure 3A, Table S5). However, no such differences were observed in the LPF treatment (Figure 3A, Table S5). There were no differences in individual $\mathrm{C}$ and $\mathrm{N}$ content between populations from the evolution experiment and neutrally evolved ancestral populations (Figure 3B, Figure S4, Table S5). In the HPF treatment, populations with a HP selection history were characterized by lower body $\mathrm{C}: \mathrm{P}$ and $\mathrm{N}: \mathrm{P}$ than the neutrally evolved ancestral population (Figure 3C, Figure S4, Table S5). In contrast, no such differences were found for the LP-selected populations in either food treatment.

All populations in the HPF compared to the LPF treatment had significantly lower individual $C$ content, $C: P, C: N$, and N:P, and higher $P$ and $N$ contents (Figure 3, Table S7, Figure S4). Populations with HP and LP selection histories did not differ in elemental content or ratios in either food quality treatments.

\section{Population growth rate per unit body $P$}

In the LPF treatment, populations with an LP selection history had greater PGR per unit body $P$ than the neutrally evolved ancestral populations (Figure 4, Table S5). No such difference was found for populations with an HP selection history in the 


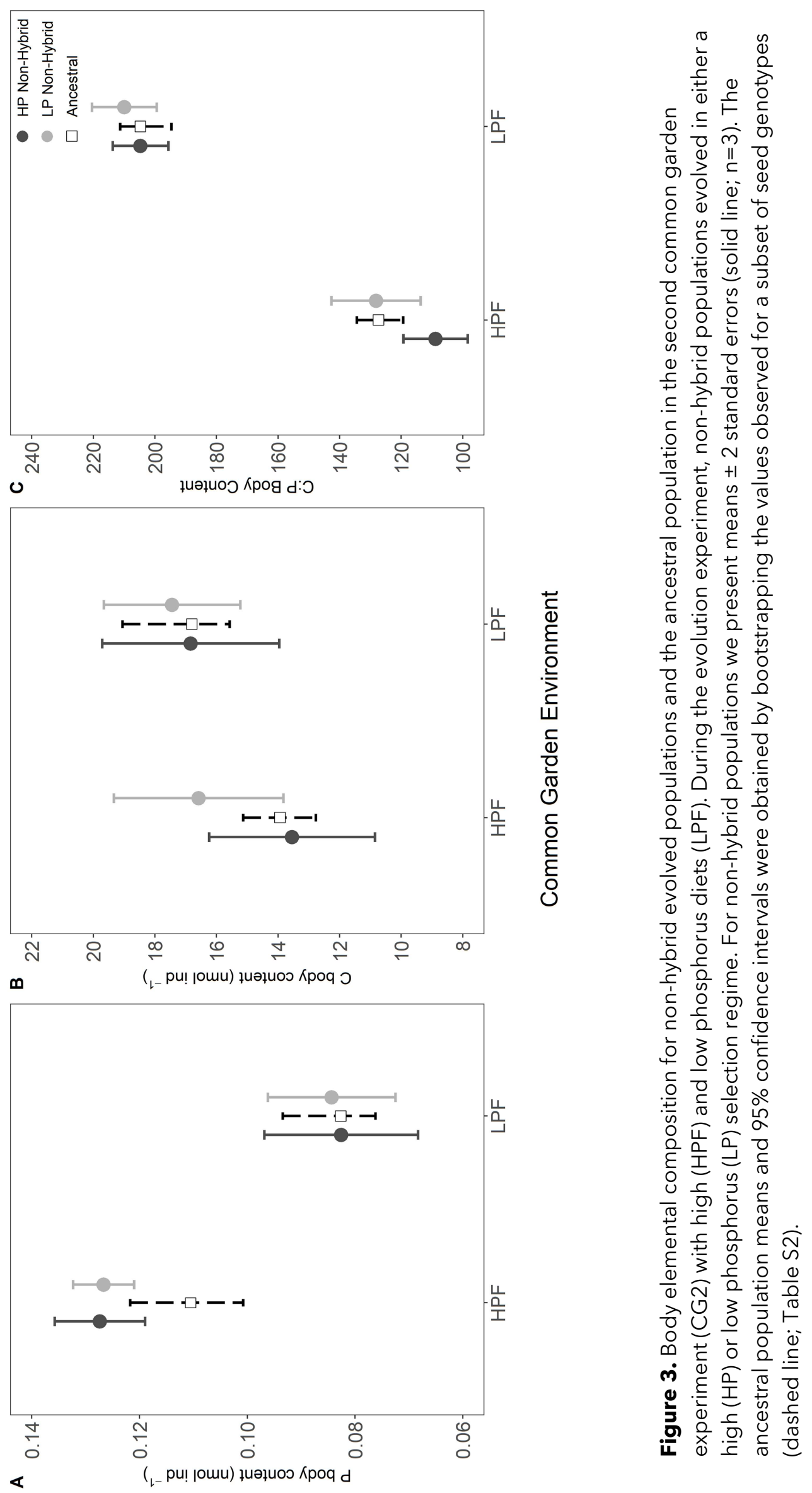

$60 \mid$ Chapter 3 
HPF treatment (Figure 4, Table S5). The LPF treatment overall reduced PGR per unit body $\mathrm{P}$ but there was a marginally significant interaction between selection history and food treatment (Figure 4, Table S7): LP-selected populations had significantly greater PGR per unit body $\mathrm{P}$ than HP-selected populations in the LPF treatment (post hoc test, $p=0.038$ ), but not in the HPF treatment.

\section{Hybrid Populations in Common Garden Experiments}

Across food quality treatments, the PGR of hybrids was significantly greater than the non-hybrids (Figure 2, Table S8) and ancestral populations (Figure 2, Table S5). PGR was similar for hybrid populations regardless of selection regime in both food treatments (Figure 2, Table 1).

There was no evidence for differences in elemental content and composition between hybrids and ancestral populations, though N:P tended to be higher in hybrids (Table S5, Figure S4C). Populations of hybrids did not differ from nonhybrids with respect to body stoichiometry in both food quality treatments (Table S8, Figure S4 and S5).

Hybrid populations had a strikingly lower fraction of sexual adults than both nonhybrid and ancestral populations in CG1 and the life history experiment (Table S5 and S9, Figure S5). In CG1, on average only $5 \%$ of the mature individuals in hybrid populations were sexual compared to $30-50 \%$ in the other populations. In the LPF life history experiment, hybrids had a $12.5 \%$ propensity for sex compared to 20 $60 \%$ in the non-hybrid and ancestral populations (Table S5). 


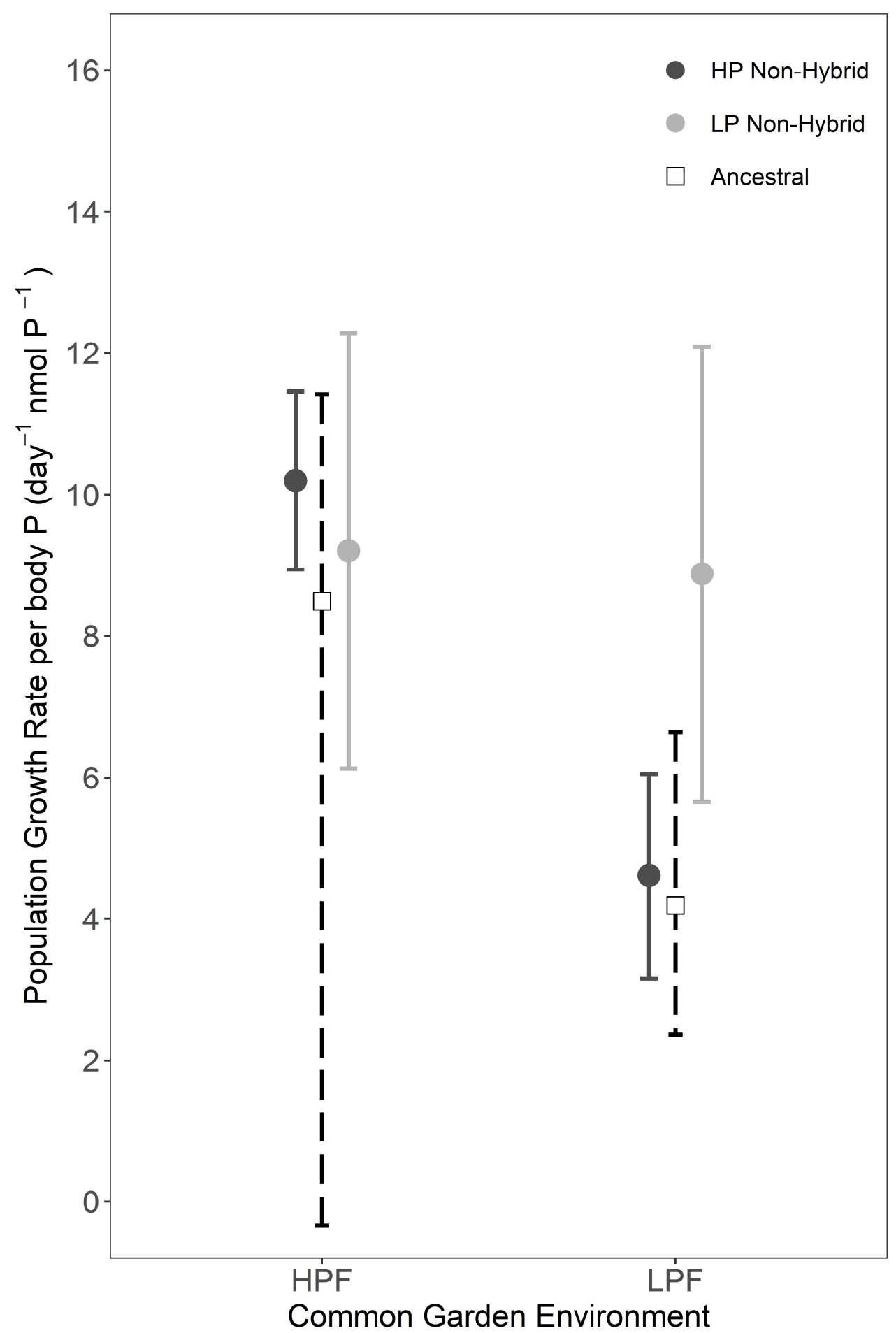

Figure 4. Population growth rate per unit body $P$ for non-hybrid evolved populations and the ancestral population with high (HPF) and low phosphorus diets (LPF). Body $\mathrm{P}$ is expressed per individual. During the evolution experiment, non-hybrid populations evolved in either a high (HP) or low phosphorus (LP) selection regime. For non-hybrid populations we present means \pm 2 standard errors (solid line; $n=3$ ). The ancestral population means and $95 \%$ confidence intervals were obtained by bootstrapping the values observed for a subset of seed genotypes (dashed line; Table S2). 


\section{Discussion}

Our study demonstrates that the GRH has the potential to predict microevolutionary responses of populations under selection for fast growth. However, this success is largely contingent on the stoichiometric context under which such selection takes place, and the genetic background of dominant genotypes (i.e., hybrid vs. non-hybrid). Consistent with GRH predictions, nonhybrid populations selected for fast population growth under P-rich conditions evolved a higher body P-content, and lower N:P and C:P than their ancestral populations. Conversely, under P-poor conditions, non-hybrid populations did not evolve higher P-content, yet they evolved higher PGRs than the ancestral populations. Furthermore, populations dominated by hybrid genotypes had higher PGRs than those dominated by non-hybrids. The latter was unrelated to differences in body P-content and was consistent across food quality treatments. Thus, although the evolutionary trajectories of non-hybrid populations selected in HPF conditions align well with GRH predictions, it appears that increased PGRs may also be achieved through pathways that do not change body stoichiometry.

\section{Evolutionary Response of Non-Hybrid Populations to a Phosphorus Rich Diet} In non-hybrid populations, selection for fast population growth under P-rich conditions resulted in simultaneous evolution towards increased PGR and individual P-content, consequently reducing body C:P and N:P. These observations strongly support our GRH-derived prediction that selection for fast population growth should promote P-rich genotypes with high somatic growth rates (Fig 1). Admittedly, in this experiment we selected for elevated population growth, and not somatic growth, due to the logistical difficulties of directly selecting on the latter (e.g., small size and rapid generation times of the model organism). However, in zooplankton in general, and B. calyciflorus specifically, SGR and PGR tend to covary consistently (Lampert \& Trubetskova 1996; Zhou \& Declerck 2019). Somatic P-content is determined by consumption rates (SuzukiOhno et al. 2012), the efficiency of P-assimilation (Urabe et al. 2018), and Pretention (Frisch et al. 2014). Selection for fast population growth under HPF conditions likely selected for genotypes that were best at assimilating the abundant $\mathrm{P}$ of their food, making it available for ribosome production, ultimately facilitating rapid protein synthesis.

\section{Evolutionary Response of Non-Hybrid Populations to a Phosphorus Poor Diet}

When exposed to P-deficient food resources, P-rich, fast growing organisms have been observed to experience greater reductions in performance compared to relatively P-poor, slow growing organisms (Sterner \& Hessen 1994). Therefore, in a 
P-poor environment, genotypes that are reliant on increased P-content to achieve elevated growth rates are not expected to be favoured. Consistently, in our study, non-hybrid populations selected in the P-poor environment evolved increased PGR without a concomitant increase in body $P$ in their home environment (i.e., when fed LPF).

The observed increase in PGR per unit body $P$ in the P-poor environment indicates the evolution of traits other than those assumed relevant in the GRH. For example, under these environmental conditions, fast population growth may be achieved through increased metabolic efficiency of $P$ or reduced costs of excess $C$ (Hessen \& Anderson 2008). Selection may also benefit genotypes that are better at coping with negative indirect, non-stoichiometric, effects of P-limitation (Rothhaupt 1995; Zhou et al. 2018; Zhou \& Declerck 2020), such as changes in algal morphology (van Donk et al. 1997) or biochemical quality (Muller Navarra 1995; von Elert et al. 2003). Although increased PGR per unit body P could be generated by shifts in life history strategy, such as a reduced propensity for sex (Becks \& Agrawal 2013), our first common garden and life history experiment revealed no such changes.

\section{Evolution of Reaction Norms}

Selection history mediated the response of populations to food quality. The performance of all populations was reduced under P-poor conditions (Figure 2), however, LP-selected populations showed a smaller reduction in growth rate in the LPF compared to HPF (37\%) than that of HP-selected or neutrally evolved ancestral populations (69 and $71 \%$ respectively). Adaptation to selection for fast growth under HPF conditions provided HP-adapted populations no advantages compared to their ancestral populations under LPF conditions. In contrast, adaptation under LPF conditions tended to result in an enhanced performance similar to that of the HP adapted populations under HPF conditions. Thus, contrary to the expectations of local adaptation, traits evolved under LPF conditions also conveyed a performance benefit when $P$ was not limiting, while the opposite was not true. Intriguingly, under HP conditions, the increased performance of LP-adapted populations was accompanied with an augmented individual $P$ content compared to ancestral populations. This suggests that adaptation to P-limitation involved traits linked to P-metabolism, although changes to body stoichiometry did not become apparent under LP-conditions.

Overall, performance differences among populations in their home environments (i.e., LP and HP adapted populations in LPF and HPF treatments, respectively) were less pronounced than expected based on the negative effect of $\mathrm{P}$-limitation. This was, entirely due to the evolutionary response of the LP-adapted populations. Instead of local adaptation, we observed a pattern indicative of counter gradient 
variation with a strong genotype by environment interaction (Conover et al. 2009). Zooplankton populations have shown similar evolutionary responses to Plimitation. For example, Frisch et al. (2014) resurrected genotypes from periods of high and low resource availability. Genotypes originating from oligotrophic periods before European settlement had higher growth performances under Ppoor conditions than genotypes from recent, more eutrophic periods. In contrast, no consistent differences between genotypes were found in P-rich food. Declerck et al. (2015) performed an evolution experiment with rotifers that selected for competitive ability under LPF and HPF conditions. They demonstrated that when exposed to a LPF treatment, populations with an LP selection history realized a higher food exploitation efficiency than populations with an HP selection history. Conversely, in the HPF treatment, LP-adapted populations showed a similar performance as HP-adapted populations. Overall, the remarkable consistency of reaction norm responses of zooplankton consumers to P-limitation, despite widely different contexts, suggests that adaptation to P-limitation may represent a relatively general but underappreciated example of cryptic evolution in zooplankton (Kinnison et al. 2015). Furthermore, it is remarkable that none of these studies on adaptation to P-limitation have found trade-offs with performance under P-sufficient conditions.

\section{Hybrid Populations}

Hybrid populations had greater PGRs than ancestral and most non-hybrid populations under all conditions. Higher PGRs were more strongly associated with a lower propensity for sex than with a higher individual P-content (Figure S5B, S5E). In rotifers, a reduction in the propensity for sex enhances clonal PGRs by reducing demographic costs associated with sexual reproduction (Serra \& Snell 2009; Becks \& Agrawal 2013). The fact that hybrids did not dominate all populations despite their relatively high PGRs is likely because stochasticity had an important role in determining the genotypic composition of the populations of our evolution experiment. Nevertheless, our hybrid results demonstrate that PGRs may be determined by traits other than individual P-content, and illustrate some limitations of the GRH as a predictive microevolutionary framework.

Ecological Implications of Rapid Adaptation to Selection for Fast Growth and its Dependency on Stoichiometric Context

Herbivores are important for trophic dynamics (Trussell \& Schmitz 2012).

Experiments have revealed that microevolutionary adaptation in consumer populations can affect higher trophic levels (e.g., Fryxell et al. 2019), however, the eco-evolutionary implications of adaptation in a stoichiometric context has received limited consideration (Yamamichi et al. 2015). Humans are increasingly 
impacting nutrient supply rates and the stoichiometry of primary producers in aquatic systems (Stockner et al. 2000; Smith \& Schindler 2009). To improve our understanding of anthropogenic impacts on food web ecology, further integration of stoichiometry and the study of eco-evolutionary dynamics is needed, including feedbacks between trophic levels (Hall 2009; Wood et al. 2018).

The results of this study demonstrate the predictive power of the GRH. Yet, its application may be limited to environments that select for fast somatic growth, where $P$ is not limiting, and population growth is density independent (i.e., resources are non-limiting). Such conditions are similar to what natural consumer populations may be experiencing when colonizing novel habitats (MacArthur \& Wilson 1967), or when being exposed to high levels of predation (Walsh \& Reznick 2008), short growing seasons (Elser et al. 2000c; Walsh \& Post 2011), or frequent disturbances (e.g., droughts, disease outbreaks; Lachish et al. 2009; Vanschoenwinkel et al. 2010). Our observation of rapid evolutionary responses by consumers to such a selection regime may have important yet understudied ecoevolutionary implications for higher trophic levels and food web functioning (e.g., by enhancing secondary productivity). For example, increased PGRs in primary consumers may at first partially compensate for mortality rates imposed by predators. Simultaneously, increases of herbivore P-content, concomitant with their increased productivity, may contribute to an increased resource base for predators. The resulting enhancement of predator productivity (Boersma et al. 2008; Malzahn et al. 2010; Schoo et al. 2012) may ultimately result in a strengthened top-down control of primary consumers (Hall et al. 2007). To explore the eco-evolutionary consequences of the adaptive responses observed in our study and its dependency on stoichiometric context, there is clearly a need for more dedicated experiments and modeling efforts.

\section{Conclusion}

Our results demonstrate the validity of the GRH as a framework for the prediction of microevolutionary responses of populations to selection for fast growth. At the same time, they also demonstrate the limits of its application, as predictions proved strongly dependent on the environmental context and genetic background of genotypes under consideration. Although the evolution of higher PGRs coincided with increased individual P-content for populations selected with HPF resources, the evolution of higher PGRs in hybrid populations and those selected with LPF resources was achieved through other mechanisms. An additional limitation is that the GRH may only be applicable to populations that are subject to selection for fast somatic growth where resources are abundant and where negative feedbacks between consumer population growth and its resources are negligible. 
This study clearly demonstrates the importance of stoichiometric context to evolutionary processes (Kay et al. 2005). In experimental work, the selection history of genotypes is almost never considered although it may be pivotal in explaining apparent discrepancies between different studies (e.g., DeMott et al. 1998; Hood \& Sterner 2014; Sherman et al. 2017). For this reason, we advocate for the inclusion of organismal selection histories into the ecological stoichiometric framework whenever possible. Furthermore, the observation of rapid evolution in both body stoichiometry and population demography in this study suggests the need for more investigations of the impact of both apparent and cryptic evolution in herbivore consumers on trophic dynamics.

\section{Acknowledgements}

This work was supported by grant $n^{\circ} 823.01 .011$ of the Earth and Life Sciences Division (ALW) of the Netherlands Organization for Scientific Research (NWO). We wish to thank Erik Kruitbosch for laboratory assistance throughout the study, Nico Helmsing for carrying out nutrient analyses and Michaela Brehm for help with microsatellite analyses. We also thank Cam Hudson, Blake Matthews and Celia Symons for constructive comments on the manuscript. 


\section{Supplementary Materials}

\section{Appendix S1: Additional Methodological Information}

Maintenance of Seed Genotype Batch Cultures

We maintained seed genotypes in $1.5 \mathrm{~mL}$ wells of tissue culture plates under constant light and at room temperature throughout the duration of the study. Theses cultures had a density of 10-15 rotifer individuals $/ \mathrm{mL}$, and we provided them $1 \mathrm{~mL}$ of an algal suspension comprised of nutrient replete Chlamydomonas reinhardtii and nutrient-free WC media (Kilham et al. 1998) at a concentration of $\sim 1000 \mu \mathrm{mol} \mathrm{L-1} \mathrm{C}$. Three times a week, we moved ten individuals from each culture into new algae suspension.

\section{Microstatellite Analysis}

Rotifer genotyping was performed on DNA extracts from single rotifers using the HotSHOT method (Montero-Pau et al. 2008). In each panel we used, for a $5 \mu \mathrm{l} \mathrm{PCR}$ reaction, $0.5 \mu \mathrm{l}$ template DNA, $0.1 \mu \mathrm{l}$ from each primer (from $10 \mathrm{pmol} / \mu \mathrm{l}$ concentration), $(2.5 \mu$ l Master Mix of the QIAGEN Multiplex PCR kit (2× stock concentration), and $0.8 \mu \mathrm{l}$ Milli-Q water. PCR thermocycler conditions involved an initial denaturation of $95^{\circ} \mathrm{C}$ for 15 min required to activate the HotStarTaq DNA polymerase of the QIAGEN Multiplex PCR kit. The next step was 30 cycles of 30 sec at $95^{\circ} \mathrm{C}, 90 \mathrm{sec}$ at $56^{\circ} \mathrm{C}$, and $60 \mathrm{sec}$ at $72^{\circ} \mathrm{C}$. A final elongation step of $30 \mathrm{~min}$ at $60^{\circ} \mathrm{C}$ completed the amplification. The PCR amplicon was then diluted 1:20 with Milli-Q water and $1 \mu \mathrm{l}$ from this dilution was then mixed with $8.8 \mu \mathrm{l}$ of formamide and $0.2 \mu \mathrm{l}$ of GeneScan ${ }^{\mathrm{TM}} 500 \mathrm{LIZ}^{\mathrm{TM}}$ size standard (Applied Biosystems, CA) prior to loading $1 \mu$ into an ABI Prism 3130 DNA Analyzer (Applied Biosystems, CA). Samples were run for $30 \mathrm{~min}$ at $15000 \mathrm{~V}$ using $36 \mathrm{~cm}$ capillaries. Allele calling was performed with the software GeneMapper ${ }^{\circledR}$ v. 4.0 using GS500 (-250) LIZ as a size standard. Peak threshold was set to 50 rfu for each dye but two different users also did manual inspection and correction, when necessary, of the allele calls.

Multilocus genotype (MLG) assignment and analyses of genetic relationship were conducted with GenoDive v.2.b27 (Meirmans \& Van Tienderen 2004), a program designed for the analysis of genetic diversity of clonal organisms.

Before the evolution experiment, we performed a microsatellite analysis on two individuals from each of the thirty seed genotypes to establish their multilocus genotype (MLG) using the microsatellite primers as described in Declerck et al. (2015). At the end of the evolution experiment (day 35), we extracted DNA from ten haphazardly chosen individuals from each of the experimental populations. We then performed microsatellite analysis to determine the genetic composition 
of each population. If we detected more than one MLG within a population (i.e., HP1 and LP7) an additional ten individuals were sequenced.

\section{Algae Cultures and Food Preparation}

We used the green algae Chlamydomonas reinhardtii as food source for the rotifers. To produce high phosphorus algae ('HPF': molar C:P ratio $121 \pm 11.9 \mathrm{SE}$ ) we used media with $65 \mu \mathrm{mol} \mathrm{L} \mathrm{L}^{-1} \mathrm{P}$ under $\approx 40 \mu \mathrm{mol}$ quanta $\mathrm{m}^{-2} \mathrm{~s}^{-1}$ of continuous light, while low phosphorus algae ('LPF': molar C:P $671 \pm 9.9 \mathrm{SE}$ ) received media with $15 \mu \mathrm{mol} \mathrm{L-1} \mathrm{P}$ and under $\approx 120 \mu \mathrm{mol}$ quanta $\mathrm{m}^{-2} \mathrm{~s}^{-1}$ of continuous light. For batch cultures and all experiments, we prepared the algal suspension by estimating carbon content using biovolume (Multisizer 3 Coulter Counter, Beckman Coulter) and diluting the algae with nutrient-free WC media to the desired concentration. After dilution, we added a vitamin mixture (Kilham et al. 1998) at a concentration of $1 \mathrm{~mL} \mathrm{~L}^{-1}$.

\section{Demographic Classification}

To assess population demography we counted preserved samples for each replicate of CG1 using a MZ16 Leica stereomicroscope at 25X magnification. Individuals were classified as one of the following: females without eggs, females with sexual eggs (male eggs and diapausing eggs), and females carrying parthenogenetic eggs. We also recorded the total number of parthenogenetic and diapausing eggs (loose and attached to female).

\section{Rotifer Culturing for Quantification of Elemental Composition (CG2)}

We used a different culturing method in CG2, as quantifying rotifer elemental body composition requires many individuals in the same body condition. In both common gardens we allowed populations to grow exponentially under ad libitum food concentrations. In CG1 this was achieved in a constant culture volume by daily reducing population densities so that food did not become limiting. In CG2, this was achieved by allowing population size to grow while increasing the culture volume proportionally on a daily basis so that population density remained constant (20 rotifers $/ \mathrm{mL}$ ) and food abundant.

To initiate CG2 we used individuals from the cultures that were maintained throughout the evolution experiment and CG1. Sixty individuals from each evolved population and seed genotype were randomly allocated to either an HPor LP-food culture. Initially, we provided all experimental units with $3 \mathrm{~mL}$ of food suspension at a concentration of $1550 \mu \mathrm{mol} \mathrm{L-1} \mathrm{C}$. We assessed population size daily by taking a $1 \mathrm{~mL}$ sample and counting all individuals present. Population size 
was then used to determine the subsequent culture volume and individuals were transferred to the fresh medium using an $80 \mu \mathrm{m}$ mesh. In this way, we scaled up culture volumes until we reached a population size of 4000 individuals. We sampled populations for elemental quantification after three consecutive days of constant population growth while keeping population densities constant. We obtained replicates by subsampling 100 individuals from the large population and restarting the culture. We waited at least seven days between sampling replicates, allowing for three asexual generations at a minimum, thereby reducing the impact of maternal effects and allowing for replicates to become independent. All populations and seed clones were represented by three replicates.

\section{Determination of Elemental Composition}

We determined rotifer $\mathrm{C}$ and $\mathrm{N}$ contents using a FLASH 2000 organic element analyzer (Interscience B.V., Breda, Netherlands), and P content with a QuAAtro segmented flow autoanalyzer (Beun de Ronde, Abcoude, Netherlands). For each of these analyses we used a sample of 100 individuals with a single parthenogenetic egg. Prior to harvesting for elemental analysis, we manually isolated rotifers and transferred them to nutrient free WC media for one hour to allow for the emptying of gut.

\section{Life History Experiment in LP Food}

Alternative life history strategies may be favored in response to selection for fast growth in P-poor environments, thus we conducted a life history experiment in low-P food with the populations used in the second common garden (Table S2). Prior to the experiment individuals from batch cultures were isolated in $1 \mathrm{~mL}$ wells of tissue culture plates, provided with an LP-diet $(1550 \mu \mathrm{mol} \mathrm{L}-1 \mathrm{C})$. The third generation was used for the experiment to minimize maternal effects (Zhou \& Declerck 2020). During the life table 15-18 individuals from each population were monitored every two hours from birth until the production of the first juvenile or until confirmed as carrying a sexual egg.

\section{Appendix S2: Additional Data Analysis Information}

\section{Evolution Experiment}

To explore temporal trends in population growth rate over the course of the evolution experiment for the two food quality treatments we fit both a piecewise and linear regression model. The model used for interpretation was chosen with the Akaike Information Criterion (i.e., with $\triangle \mathrm{AIC}<$ approximately 2; Burnham \& Anderson 2004). The piecewise regression was conducted using the package 
'segmented' (Muggeo 2008), and the initial 'breakpoint' of the model was determined after a visual inspection of the plotted data. Davies test provided by the segmented package was applied to determine the significance of differences between slopes. Observations of population growth rate from all seven populations in each selection history were used for this analysis.

\section{Trait Value Simulation of Neutrally Evolved Ancestral Populations and}

\section{Comparison to Populations Evolved in the Evolution Experiment}

For each trait in both common garden treatments we constructed a distribution of differences in mean trait values between a simulated 'neutrally evolved' ancestral population (i.e., change in phenotype due to drift associated with the repeated subsampling of small populations) and the mean of values drawn from a distribution representing the populations at the end of the evolution experiment. For the neutral evolution simulations, we subjected virtual ancestral populations to exactly the same manipulations as those that were experienced by the real populations throughout the evolution and common garden experiments (see illustration below). Neutral evolution of ancestral populations was simulated and compared to the evolved populations 10,000 times according to the following steps:

1. Generate 30 seed genotypes by drawing from a normal distribution with the same mean and variance of the trait as measured for the subset of seed genotypes that were characterized in the common garden experiment.

2. Generate three replicate ancestral populations composed of two individuals of each of the 30 seed genotypes.

3. In each of these replicate populations, allow all genotypes to grow at identical rates, equal to what was observed in each of the food quality treatments (i.e., 0.85 and 0.267 day $^{-1}$ in the HP and LP treatments, respectively). As in the experiment, at 24 hours intervals, 60 individuals are randomly selected to restart the populations. Changes in clonal frequencies within a replicate overtime (i.e., evolution) will thus only be due to chance events associated by the random selection of a subset of individuals during each transfer.

4. As in the real evolution experiments, allow populations to evolve during 36 time intervals.

5. After 36 time intervals, subsample each of the evolved populations in the same way as what was done to start technical replicates for populations as in the common garden experiment: for PGR and population structure randomly allocate 40 individuals from day 36 to four populations. For 
elemental traits we simulated no technical replicates to match the design of the second common garden experiment.

6. Identify the genotype of each individual in each population and assign its corresponding trait value. For each population, calculate the weighed mean trait value across genotypes with genotype weights corresponding to their relative abundance.

7. The trait value for the ancestral population is calculated as the mean of the three replicate populations (MEAN $N_{\text {neutr_sim }}$ ).

8. For each selection history (LP-selected, HP-selected and hybrid) draw three values from a normal distribution with the same mean and variance as observed for the population level traits measured for the corresponding selection history in the common garden experiment. Take the mean of these three traits (MEAN obs).

9. Calculate the difference $(\triangle M E A N)$ between $M E A N_{\text {neutr_sim }}$ and $M E A N_{\text {obs. }}$.

The frequency distribution of 10,000 $\triangle M E A N$-values was used to determine the probability that the observed trait change of the evolved populations differ from a neutrally evolved ancestral population. If $97.5 \%$ of the simulated differences were either all larger or smaller than zero (i.e., no difference between the observed and the neutrally evolved population), then the trait values observed in the evolved populations were considered significantly different from neutral expectations. $\mathrm{P}$ values were calculated according to the following equation, where $X$ is the simulated distribution:

$$
2 * \min \{P(X \leq 0) \mid P(X \geq 0)\}
$$

Visual representation of the simulation for ancestral populations (next page). Subscripts S, A, and R stand for seed genotype, ancestral population replicate, and common garden pseudo replicate, respectively.

A. Trait values for 30 genotypes are generated from a normal distribution with same mean and variance as observed for the seed genotypes. Each circle represents a single genotype with its own trait value. Colored circles represent three of the genotypes drawn from the normal distribution.

B. Three replicate ancestral populations are each composed of two individuals per genotype.

C. Drift resulting from the daily random subsampling of the populations has affected the relative abundance of genotypes at the end of the evolution experiment

D. Forty individuals are randomly allocated (without replacement) to the four (pseudo)replicate populations of the common garden experiment. The trait value for the ancestral population is calculated as the mean of these four (pseudo)replicate populations. 


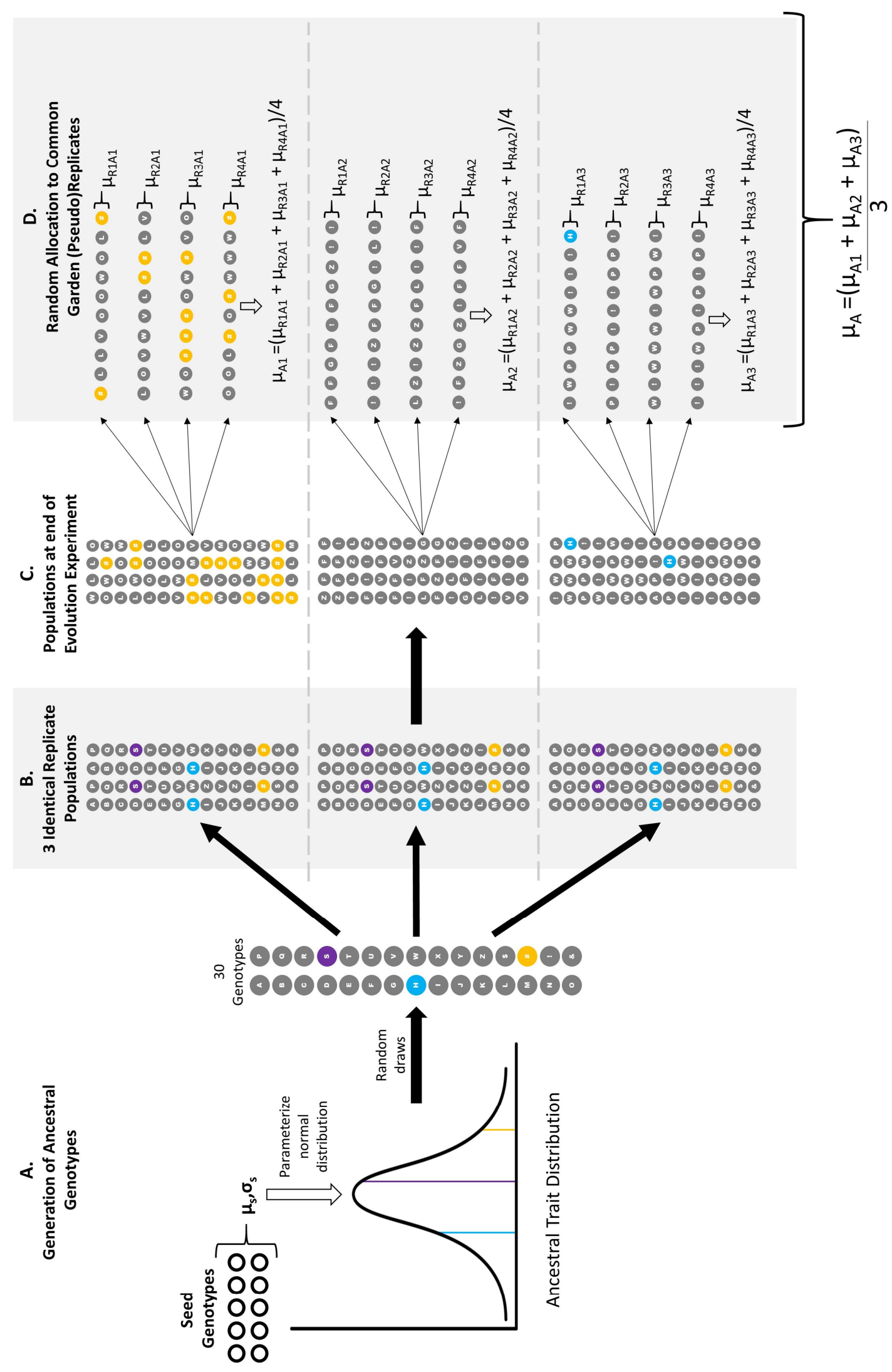

An experimental test of the Growth Rate Hypothesis as a predictive framework for microevolutionary adaptation | 73 


\section{Supplementary Tables}

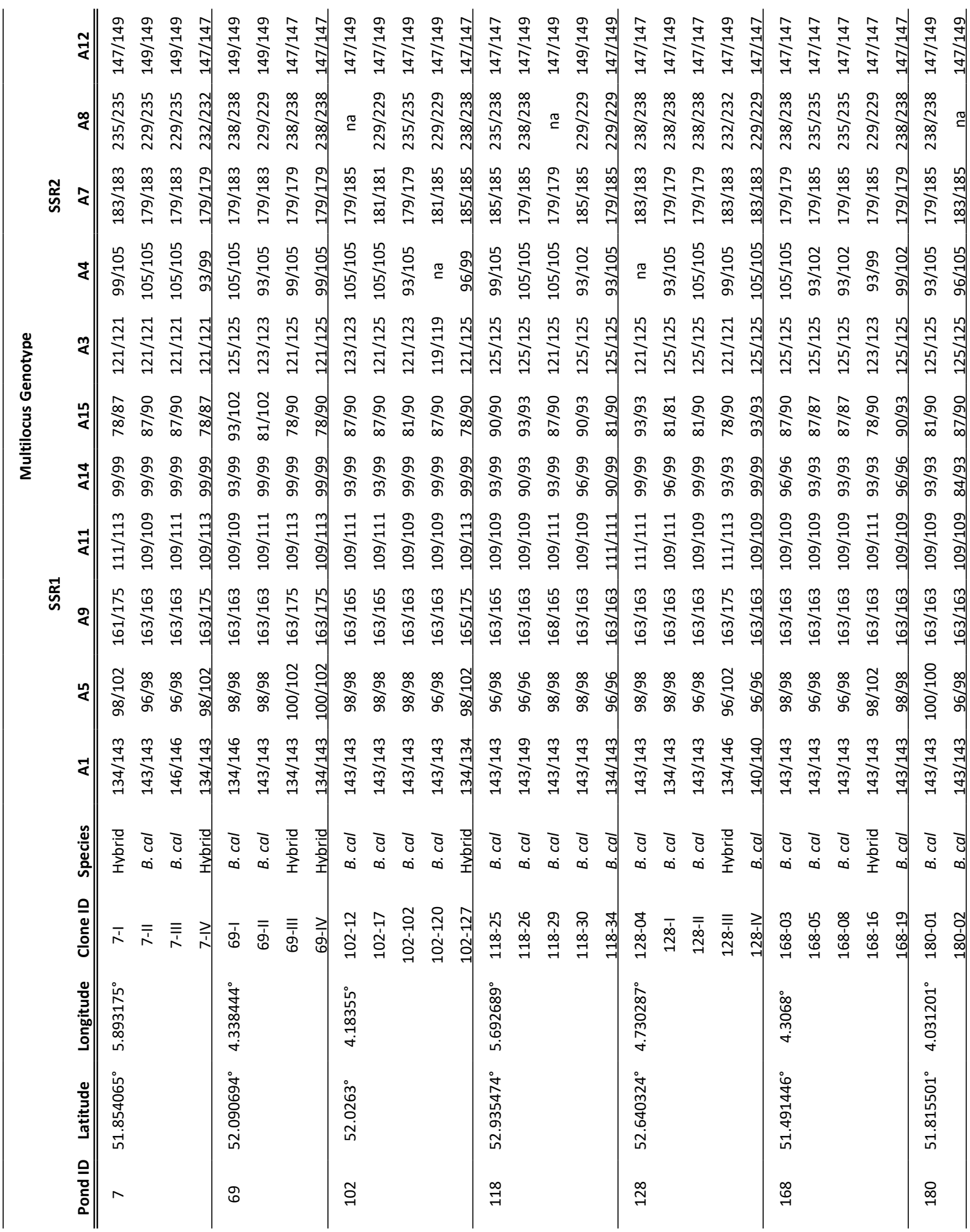

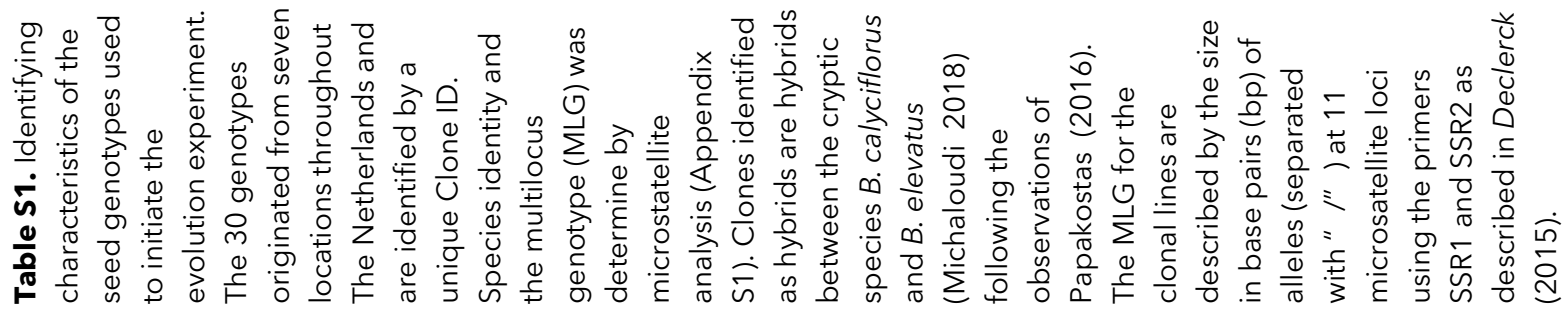




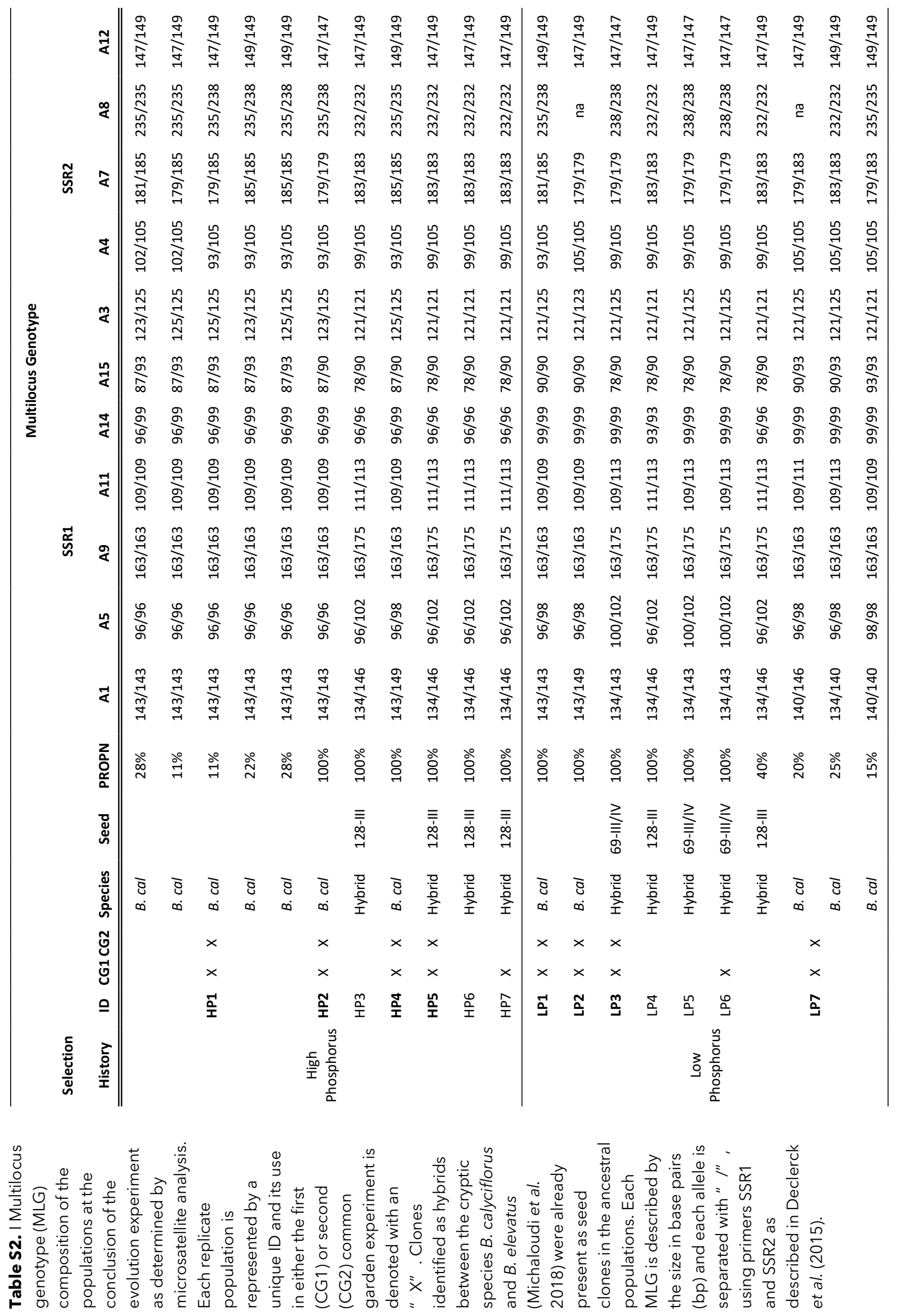

An experimental test of the Growth Rate Hypothesis as a predictive framework for microevolutionary adaptation | 75 
Table S3. Comparison of linear and piecewise (PW) regression models describing the course of population growth rate over time in the evolution experiment for populations in low and high phosphorus food treatments ( $n=7$ populations). Models in bold are those selected for interpretation based on AIC. Slopes differing significantly from 0 are indicated with an *. Davies test $p$-value indicates a significant difference between the two slopes of the piecewise regression models. Seg. is segmented and $\mathrm{Cl}$ is confidence interval.

\begin{tabular}{cccccccccccc}
\hline $\begin{array}{c}\text { Food } \\
\text { Quality }\end{array}$ & Model & df & AIC & $\begin{array}{c}\text { Seg. } \\
\text { at }\end{array}$ & Cl & Slope & $\mathbf{9 5 \%}$ Cl & Slope & $\mathbf{9 5 \% ~ C l}$ & $\begin{array}{c}\text { Davies } \\
\text { test p }\end{array}$ \\
\hline \hline High & Linear & $\mathbf{3}$ & $-\mathbf{7 . 1 5}$ & - & - & $\mathbf{0 . 0 1}$ & $\mathbf{( 0 . 0 0 5 , 0 . 0 1 4 )}$ & - & - & - \\
Phosphorus & PW & 5 & -6.08 & 5 & $(-2,12)$ & -0.04 & $(-0.156,0.77)$ & 0.012 & $(0.007,0.018)$ & 0.277 \\
\hline Low & Linear & 3 & -56.88 & - & - & -0.003 & $(-0.007,0)$ & - & - & - \\
Phosphorus & PW & $\mathbf{5}$ & $-\mathbf{6 9 . 8 6}$ & $\mathbf{1 3 . 5}$ & $\mathbf{( 9 , 1 8 )}$ & $-\mathbf{0 . 0 2 5}$ & $\mathbf{( - 0 . 0 3 9 , - 0 . 0 1 1 )}$ & $\mathbf{0 . 0 0 6}$ & $\mathbf{( 0 , 0 . 0 1 2 )}$ & $<0.001$ \\
\hline
\end{tabular}

Table S4. Estimated trait mean, standard deviation (sd), and bias-corrected accelerated $95 \%$ percentile confidence intervals (Lower $\mathrm{Cl}$ and Upper $\mathrm{Cl}$ ) based on bootstrapping $(n=1000)$ of the measured trait values for the subset of 10 seed genotypes measured in the common garden experiments. Traits were measured in the following units: Population growth rate $\left(\right.$ day $\left.^{-1}\right)$, fraction of sexual individuals (\% of total population), $C, N$ and $P$ content $\left(n m o l\right.$ individual ${ }^{-1}$ ), elemental ratios are unitless, population growth rate per body $\mathrm{P}\left(\right.$ day $\left.^{-1} \mathrm{nmol}^{\mathrm{P}}{ }^{-1}\right)$, and propensity for sex (\% of total population).

\begin{tabular}{lcccccccc}
\hline & \multicolumn{7}{c}{ HPF Treatment } & \multicolumn{3}{c}{ LPF Treatment } \\
Trait & mean & sd & Lower Cl & Upper Cl & mean & sd & Lower Cl & Upper Cl \\
\hline \hline $\begin{array}{l}\text { Population Growth } \\
\text { Rate }\end{array}$ & 0.85 & 0.55 & 0.44 & 1.12 & 0.26 & 0.29 & 0.08 & 0.44 \\
\hline $\begin{array}{l}\text { Fraction of Sexual } \\
\text { Individuals }\end{array}$ & 0.51 & 0.22 & 0.32 & 0.61 & 0.25 & 0.22 & 0.12 & 0.40 \\
\hline C & 13.94 & 1.80 & 12.78 & 15.13 & 16.80 & 2.66 & 15.57 & 19.05 \\
\hline N & 2.74 & 0.32 & 2.53 & 2.95 & 2.46 & 0.36 & 2.27 & 2.75 \\
\hline P & 0.11 & 0.02 & 0.10 & 0.12 & 0.08 & 0.01 & 0.08 & 0.09 \\
\hline C:N & 5.09 & 0.13 & 5.00 & 5.17 & 6.85 & 0.31 & 6.62 & 7.02 \\
\hline C:P & 127.37 & 11.53 & 119.33 & 134.40 & 204.75 & 12.78 & 194.61 & 211.31 \\
\hline N:P & 24.96 & 2.18 & 23.74 & 26.58 & 29.60 & 1.46 & 28.58 & 30.49 \\
\hline PGR per Body P & 8.49 & 7.35 & -0.34 & 11.41 & 4.19 & 2.67 & 2.36 & 6.64 \\
\hline Propensity for Sex & & & & & 0.50 & 0.18 & 0.38 & 0.62 \\
\hline
\end{tabular}


Table S5. Means of trait values simulated for neutrally evolved ancestral populations, with 2.5 and 97.5 percentiles. For the HP- selected $(n=3)$, LP-selected $(n=3)$, and hybrid $(n=4)$ populations we also present the mean value of the traits observed in the common garden experiments and the significance ( $p$-value) of differences between observed trait means and simulated means for neutrally evolved ancestral populations, as explained in Appendix S2. See Table S4 for units.

\begin{tabular}{|c|c|c|c|c|c|c|c|c|c|c|}
\hline \multirow[t]{2}{*}{ Trait } & & \multicolumn{3}{|c|}{ Neutrally Evolved } & \multicolumn{2}{|c|}{$\begin{array}{l}\text { Non-Hybrid } \\
\text { HP-selected }\end{array}$} & \multicolumn{2}{|c|}{$\begin{array}{l}\text { Non-Hybrid } \\
\text { LP-selected }\end{array}$} & \multicolumn{2}{|c|}{ Hybrid } \\
\hline & & Mean & $\begin{array}{c}2.5 \\
\text { Percentile } \\
\end{array}$ & $\begin{array}{c}97.5 \\
\text { Percentile } \\
\end{array}$ & Mean & $\mathbf{p}$ & Mean & $\mathbf{p}$ & Mean & $\mathbf{p}$ \\
\hline \multicolumn{11}{|l|}{ Population } \\
\hline \multirow{2}{*}{$\begin{array}{l}\text { Growth } \\
\text { Rate }\end{array}$} & HPF & 0.85 & 0.47 & 1.22 & 1.30 & 0.039 & 1.16 & 0.227 & 1.57 & $<0.001$ \\
\hline & LPF & 0.25 & 0.07 & 0.43 & 0.37 & 0.254 & 0.73 & $<0.001$ & 0.86 & $<0.001$ \\
\hline \multirow{3}{*}{$\begin{array}{l}\text { Fraction of } \\
\text { Sexual } \\
\text { Individuals }\end{array}$} & & & & & & & & & & \\
\hline & HPF & 0.51 & 0.37 & 0.66 & 0.40 & 0.248 & 0.32 & 0.373 & 0.05 & $<0.001$ \\
\hline & LPF & 0.26 & 0.12 & 0.39 & 0.19 & 0.529 & 0.31 & 0.645 & 0.02 & $<0.001$ \\
\hline \multicolumn{11}{|l|}{$\mathrm{C}$} \\
\hline & HPF & 13.94 & 12.74 & 15.15 & 13.54 & 0.784 & 16.58 & 0.076 & 15.43 & 0.596 \\
\hline & LPF & 16.78 & 15.19 & 18.35 & 16.83 & 0.978 & 17.44 & 0.632 & 16.80 & 0.984 \\
\hline \multicolumn{11}{|l|}{$\mathrm{N}$} \\
\hline & HPF & 2.74 & 2.53 & 2.95 & 2.67 & 0.788 & 3.23 & 0.058 & 3.01 & 0.639 \\
\hline & LPF & 2.46 & 2.24 & 2.67 & 2.45 & 1 & 2.58 & 0.49 & 2.36 & 0.784 \\
\hline \multicolumn{11}{|l|}{$P$} \\
\hline & HPF & 0.11 & 0.10 & 0.12 & 0.13 & 0.014 & 0.13 & 0.01 & 0.12 & 0.518 \\
\hline & LPF & 0.08 & 0.08 & 0.09 & 0.08 & 0.993 & 0.08 & 0.811 & 0.08 & 0.974 \\
\hline \multicolumn{11}{|l|}{$\mathrm{C}: \mathrm{N}$} \\
\hline & HPF & 5.09 & 5.00 & 5.18 & 5.05 & 0.635 & 5.13 & 0.549 & 5.15 & 0.423 \\
\hline & LPF & 6.85 & 6.67 & 7.03 & 6.84 & 0.966 & 6.78 & 0.565 & 7.16 & 0.104 \\
\hline \multicolumn{11}{|l|}{$C: P$} \\
\hline & HPF & 127.36 & 119.86 & 135.15 & 108.86 & 0.004 & 128.14 & 0.917 & 125.57 & 0.666 \\
\hline & LPF & 204.75 & 197.16 & 212.49 & 204.96 & 0.981 & 209.79 & 0.44 & 220.82 & 0.113 \\
\hline \multicolumn{11}{|l|}{$\mathrm{N}: \mathrm{P}$} \\
\hline & HPF & 24.97 & 23.49 & 26.42 & 21.52 & 0.001 & 24.93 & 0.964 & 24.27 & 0.48 \\
\hline & LPF & 29.60 & 28.73 & 30.46 & 29.92 & 0.776 & 31.01 & 0.191 & 30.88 & 0.048 \\
\hline \multicolumn{11}{|l|}{ PGR per } \\
\hline \multirow[t]{2}{*}{ Body P } & HPF & 8.48 & 3.59 & 13.35 & 10.20 & 0.498 & 9.21 & 0.79 & 13.54 & 0.134 \\
\hline & LPF & 4.08 & 2.68 & 5.54 & 4.61 & 0.597 & 8.88 & 0.007 & 10.86 & $<0.001$ \\
\hline $\begin{array}{l}\text { Propensity } \\
\text { for Sex }\end{array}$ & LPF & 0.50 & 0.39 & 0.61 & 0.57 & 0.266 & 0.28 & 0.209 & 0.13 & $<0.001$ \\
\hline
\end{tabular}


Table S6. Summary of generalized linear mixed model analysis testing for non-hybrid populations on the effects of diet and selection history on the fraction of sexual idividuals in the first common garden experiment (CG1). Effects of common garden diet (LPF or HPF) and population selection history (LP or HP evolved) are presented as the fixed components of the models. Significant effects are in bold (see also Figure S3).

\begin{tabular}{lcccc}
\hline & Estimate & Std. Error & Z-value & $\mathbf{P}$ \\
\hline \hline Fraction of Sexual Individuals & & & & \\
Diet & -1.2374 & 0.378 & -3.274 & $\mathbf{0 . 0 0 1}$ \\
Selection History & -0.7598 & 0.7432 & -1.022 & 0.307 \\
Diet x SH & 1.5385 & 0.5044 & 3.05 & $\mathbf{0 . 0 0 2}$ \\
\hline
\end{tabular}

Table S7. Effects of diet and selection history on the elemental content and elemental ratios of nonhybrid populatons from the evolution experiment. Data were obtained from the second common garden experiment and analyzed with linear mixed models where diet and selection history were treated as fixed factors. Significant effects are in bold. See Table 1 for abbreviations.

\begin{tabular}{|c|c|c|c|c|c|c|}
\hline & Sum Sq & Mean Sq & NumDF & DenDF & F value & $\mathbf{p}$ \\
\hline \multicolumn{7}{|l|}{ P content } \\
\hline Diet & $1.71 \mathrm{E}-02$ & $1.71 \mathrm{E}-02$ & 1 & 30.0 & 68.24 & $<0.001$ \\
\hline Selection History & $2.80 \mathrm{E}-06$ & $2.80 \mathrm{E}-06$ & 1 & 6.0 & 0.01 & 0.921 \\
\hline Diet $\times$ SH & $1.34 \mathrm{E}-05$ & $1.34 \mathrm{E}-05$ & 1 & 30.0 & 0.05 & 0.819 \\
\hline \multicolumn{7}{|l|}{ C content } \\
\hline Diet & $5.41 E+01$ & $5.41 \mathrm{E}+01$ & 1 & 24.1 & 18.07 & $<0.001$ \\
\hline Selection History & $4.91 E+00$ & $4.91 E+00$ & 1 & 4.0 & 1.64 & 0.269 \\
\hline Diet $x$ SH & $3.22 \mathrm{E}+00$ & $3.22 \mathrm{E}+00$ & 1 & 24.1 & 1.07 & 0.310 \\
\hline \multicolumn{7}{|l|}{$N$ content } \\
\hline Diet & $1.45 \mathrm{E}+00$ & $1.45 \mathrm{E}+00$ & 1 & 25.1 & 9.02 & 0.006 \\
\hline Selection History & $2.25 \mathrm{E}-01$ & $2.25 \mathrm{E}-01$ & 1 & 3.9 & 1.40 & 0.303 \\
\hline Diet x SH & $2.78 \mathrm{E}-01$ & $2.78 \mathrm{E}-01$ & 1 & 25.1 & 1.73 & 0.201 \\
\hline \multicolumn{7}{|l|}{$C: P$} \\
\hline Diet & $6.50 E+04$ & $6.50 E+04$ & 1 & 25.5 & 247.67 & $<0.001$ \\
\hline Selection History & $8.75 E+02$ & $8.75 E+02$ & 1 & 4.0 & 3.33 & 0.142 \\
\hline Diet $\times$ SH & $2.59 E+02$ & $2.59 E+02$ & 1 & 25.5 & 0.99 & 0.330 \\
\hline \multicolumn{7}{|l|}{$N: P$} \\
\hline Diet & $4.30 \mathrm{E}+02$ & $4.30 \mathrm{E}+02$ & 1 & 25.4 & 82.52 & $<0.001$ \\
\hline Selection History & $2.39 E+01$ & $2.39 E+01$ & 1 & 4.1 & 4.59 & 0.097 \\
\hline Diet $\times$ SH & $7.61 \mathrm{E}+00$ & $7.61 E+00$ & 1 & 25.4 & 1.46 & 0.238 \\
\hline \multicolumn{7}{|l|}{$C: N$} \\
\hline Diet & $2.43 E+01$ & $2.43 E+01$ & 1 & 29.0 & 197.94 & $<0.001$ \\
\hline Selection History & $2.00 \mathrm{E}-04$ & $2.00 \mathrm{E}-04$ & 1 & 29.0 & 0.00 & 0.972 \\
\hline Diet $x$ SH & $2.57 \mathrm{E}-02$ & $2.57 \mathrm{E}-02$ & 1 & 29.0 & 0.21 & 0.651 \\
\hline \multicolumn{7}{|l|}{ PGR per $P$} \\
\hline Diet & $2.63 \mathrm{E}+01$ & $2.63 E+01$ & 1 & 8 & 5.97 & 0.040 \\
\hline Selection History & $8.07 E+00$ & $8.07 E+00$ & 1 & 8 & 1.83 & 0.212 \\
\hline Diet $\times$ SH & $2.07 E+01$ & $2.07 E+01$ & 1 & 8 & 4.70 & 0.061 \\
\hline
\end{tabular}


Table S8. Summary of linear mixed effects analyses comparing traits of non-hybrid and hybrid populations based on data from the first and second common garden experiments. Effects of diet (LPF or HPF) and genetic background (non-hybrid or hybrid) are presented as the fixed components of the models. Significant effects are in bold (see also Figure S5). See Table 1 for abbreviations.

\begin{tabular}{|c|c|c|c|c|c|c|}
\hline & Sum Sq & Mean Sq & NumDF & DenDF & F value & p \\
\hline \multicolumn{7}{|l|}{ Population Growth Rate } \\
\hline Diet & $9.39 \mathrm{E}-01$ & 9.39E-01 & 1 & 8.1 & 147.29 & $<0.001$ \\
\hline Genetic Background & $4.11 \mathrm{E}-02$ & 4.11E-02 & 1 & 6.6 & 6.45 & 0.041 \\
\hline Diet x Genetic Background & $1.15 \mathrm{E}-02$ & $1.15 \mathrm{E}-02$ & 1 & 8.1 & 1.80 & 0.216 \\
\hline \multicolumn{7}{|l|}{ P content } \\
\hline Diet & $6.65 \mathrm{E}-03$ & $6.65 \mathrm{E}-03$ & 1 & 12.5 & 20.86 & $<0.001$ \\
\hline Genetic Background & $1.07 \mathrm{E}-05$ & $1.07 \mathrm{E}-05$ & 1 & 5.1 & 0.03 & 0.862 \\
\hline Diet x Genetic Background & 7.70E-06 & 7.70E-06 & 1 & 12.5 & 0.02 & 0.879 \\
\hline \multicolumn{7}{|l|}{ C content } \\
\hline Diet & $1.98 \mathrm{E}+01$ & $1.98 \mathrm{E}+01$ & 1 & 8.1 & 6.95 & 0.030 \\
\hline Genetic Background & $1.32 \mathrm{E}-02$ & $1.32 \mathrm{E}-02$ & 1 & 4.8 & 0.00 & 0.948 \\
\hline Diet x Genetic Background & $5.78 \mathrm{E}+00$ & $5.78 \mathrm{E}+00$ & 1 & 8.1 & 2.03 & 0.192 \\
\hline \multicolumn{7}{|l|}{$N$ content } \\
\hline Diet & 3.97E-01 & 3.97E-01 & 1 & 10.2 & 2.37 & 0.155 \\
\hline Genetic Background & $3.92 \mathrm{E}-03$ & $3.92 \mathrm{E}-03$ & 1 & 4.8 & 0.02 & 0.885 \\
\hline Diet x Genetic Background & $2.04 \mathrm{E}-01$ & 2.04E-01 & 1 & 10.2 & 1.22 & 0.296 \\
\hline \multicolumn{7}{|l|}{$C: P$} \\
\hline Diet & $6.46 \mathrm{E}+04$ & $6.46 \mathrm{E}+04$ & 1 & 24.0 & 240.52 & $<0.001$ \\
\hline Genetic Background & $1.38 \mathrm{E}+03$ & $1.38 \mathrm{E}+03$ & 1 & 24.0 & 5.13 & 0.033 \\
\hline Diet x Genetic Background & $8.00 \mathrm{E}+00$ & $8.00 E+00$ & 1 & 24.0 & 0.03 & 0.867 \\
\hline \multicolumn{7}{|l|}{$N: P$} \\
\hline Diet & 4.27E+02 & $4.27 \mathrm{E}+02$ & 1 & 24.0 & 79.31 & $<0.001$ \\
\hline Genetic Background & $1.08 \mathrm{E}+01$ & $1.08 \mathrm{E}+01$ & 1 & 24.0 & 2.01 & 0.169 \\
\hline Diet x Genetic Background & $1.10 \mathrm{E}+01$ & $1.10 \mathrm{E}+01$ & 1 & 24.0 & 2.05 & 0.166 \\
\hline \multicolumn{7}{|l|}{$C: N$} \\
\hline Diet & $2.36 \mathrm{E}+01$ & $2.36 \mathrm{E}+01$ & 1 & 24.0 & 184.73 & $<0.001$ \\
\hline Genetic Background & $4.21 \mathrm{E}-01$ & $4.21 \mathrm{E}-01$ & 1 & 24.0 & 3.29 & 0.082 \\
\hline Diet x Genetic Background & $1.79 E-01$ & $1.79 E-01$ & 1 & 24.0 & 1.40 & 0.248 \\
\hline
\end{tabular}


Table S9. Summary of generalized linear mixed model analyses comparing non-hybrid and hybrid populations in the first common garden. The fraction of sexual females was calculated as the number of females with sexual eggs (male and diapausing eggs) divided by the total number of mature individuals (i.e., adults with male, diapausing or amictic eggs). Effects of diet (low or high phosphorus) and genetic background (non-hybrid or hybrid) are presented as the fixed components of the models. Significant effects are in bold (see also Figure S5E).

\begin{tabular}{lcccc}
\hline & Estimate & Std. Error & Z-value & $\mathbf{p}$ \\
\hline \hline Fraction of Sexual Individuals & & & & \\
Diet & -0.4275 & 0.4174 & -1.024 & 0.306 \\
Genetic Background & -2.7169 & 0.3982 & -6.823 & $<0.001$ \\
Diet x Genetic Background & -0.3343 & 0.5313 & -0.629 & 0.529 \\
\hline
\end{tabular}

\section{Supplementary Figures}
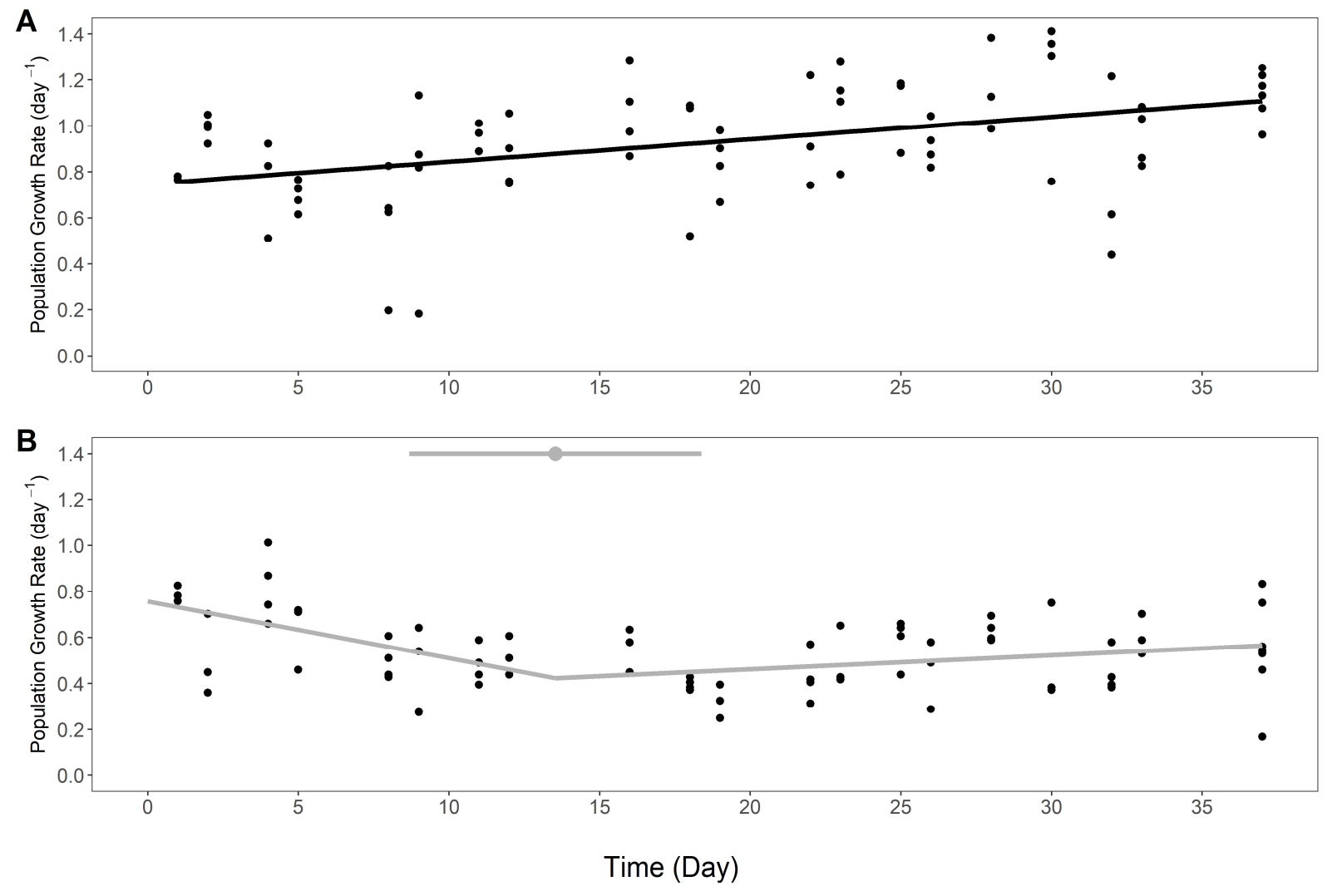

Figure S1. Temporal trends in growth rate of populations during the evolution experiment in A) HPF and B) LPF selection treatments. The line in A) represents a linear response of population growth without breakpoints (see Table S3). The grey lines in B) represent the two segments of a segmented regression analysis with a breakpoint at 13.5 days. Segment point and $95 \%$ confidence interval is represented by the grey symbol and horizontal error bar. 


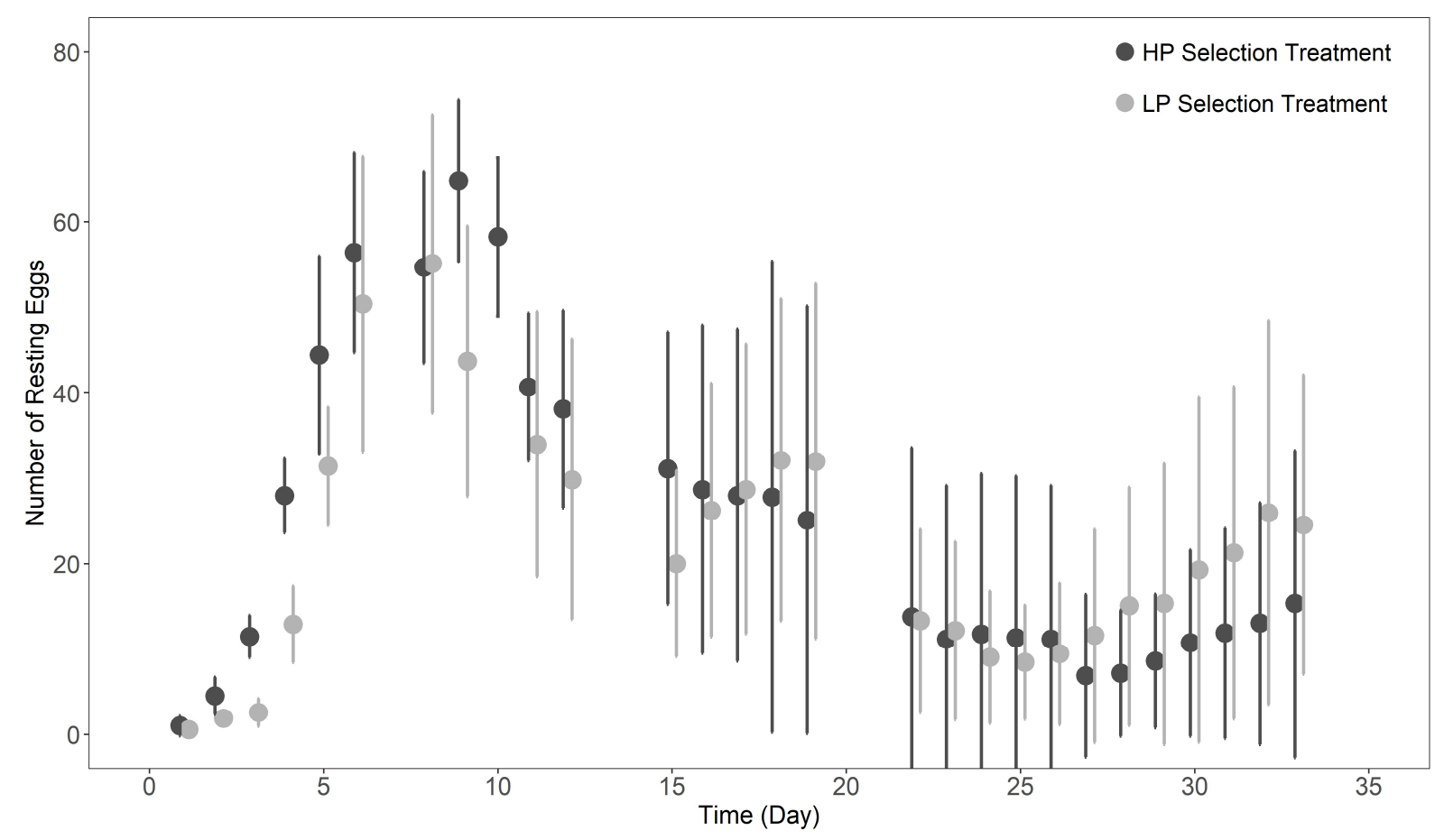

Figure S2. Number of resting eggs observed during the evolution experiment in populations of the high (HP) and low (LP) phosphorus treatments, respectively. Symbols represent means across populations $(n=7)$ and error bars represent \pm 2 standard errors.

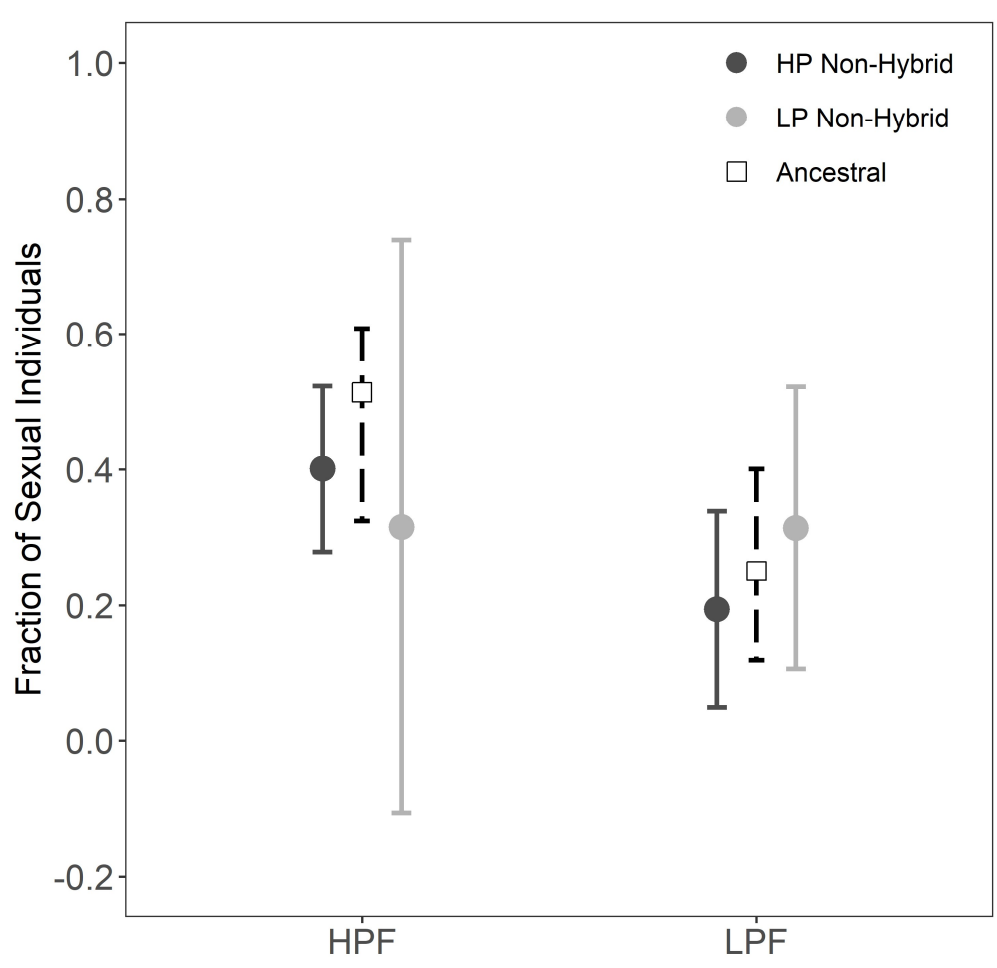

Figure S3. Fraction of sexual individuals in the non-hybrid and ancestral populations in the first common garden with high (HPF) and low phosphorus diets (LPF). Non-hybrid populations were selected in either high (HP) or low phosphorus (LP) selection regimes. The fraction of sexual females was calculated as the number of females with sexual eggs (male and diapausing eggs) divided by the total number of mature individuals (i.e., adults with male, diapausing, or amictic eggs). For non-hybrid populations we present means \pm 2 standard errors (solid line; $n=3$ ). For the ancestral population, means and $95 \%$ confidence intervals were obtained by bootstrapping the values observed for a subset of seed genotypes (dashed line; Table S2).

An experimental test of the Growth Rate Hypothesis as a predictive framework for microevolutionary adaptation | 81 

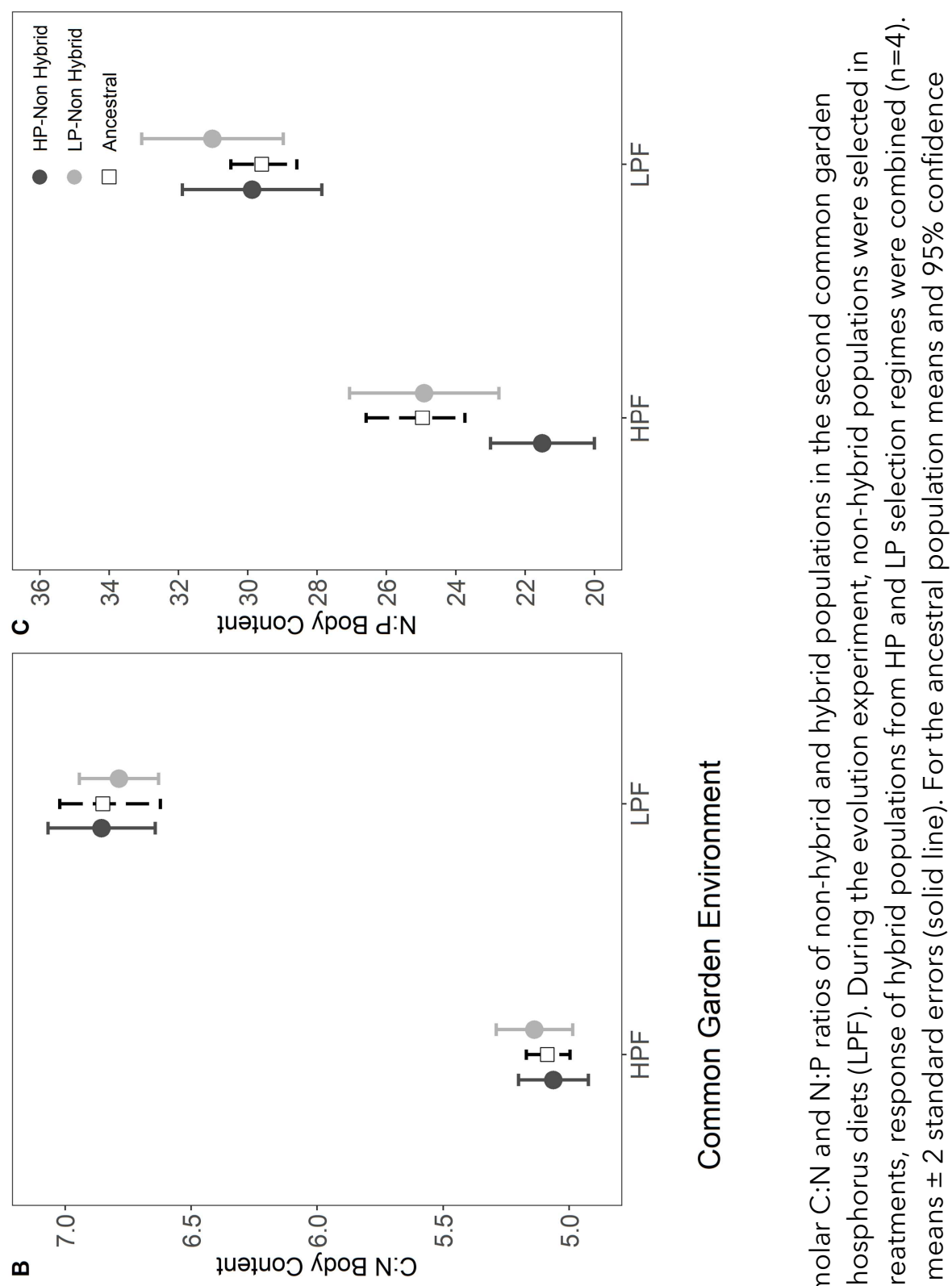

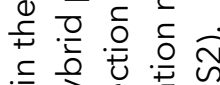

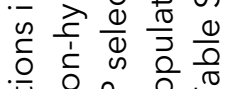

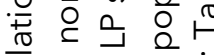
落 ¿. ह 웡 은 츤 모

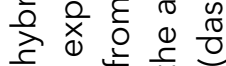
은 은

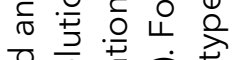

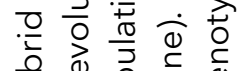
त

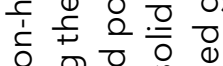

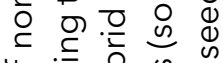
顿

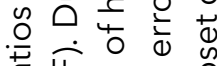

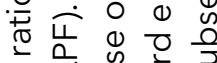

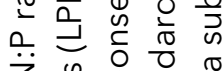

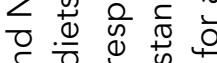

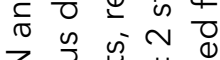

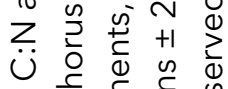
元 त 00 क 0

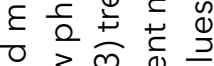

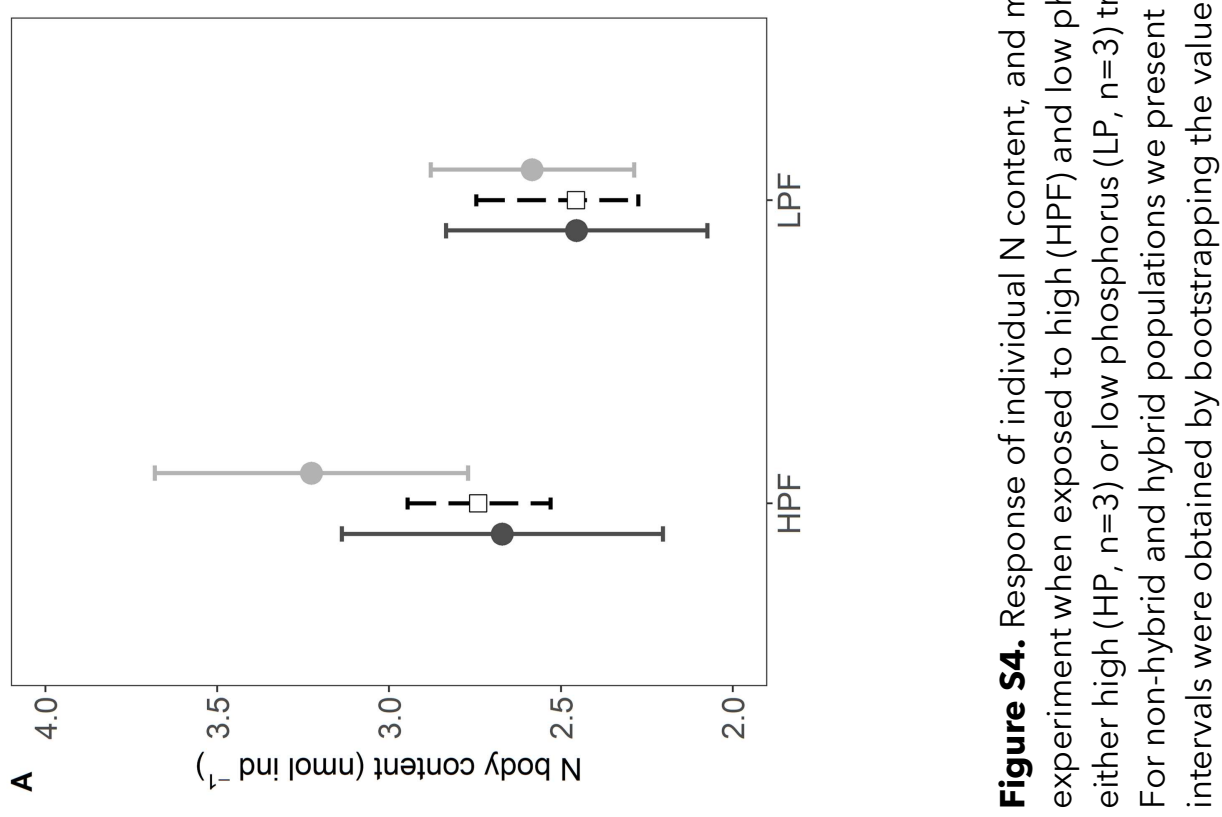



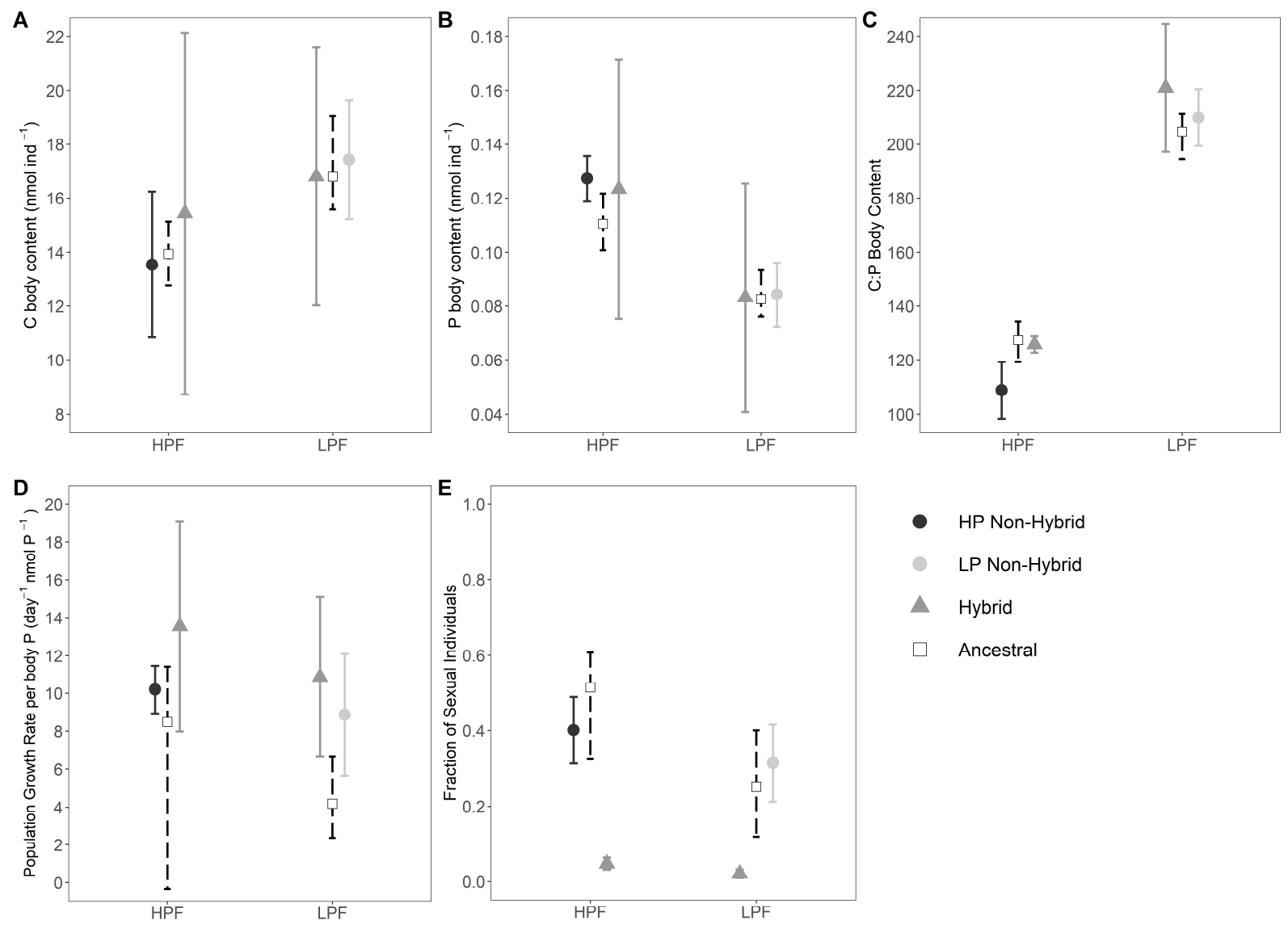

- HP Non-Hybrid

LP Non-Hybrid

A Hybrid

$\square \quad$ Ancestral

Common Garden Environment

Figure S5. Comparison of traits between non-hybrid and hybrid populations. Populations are compared in the two food quality treatments of the common garden experiments corresponding to their selection history in the evolution experiment (i.e., LP and HP-selected populations in LPF and HPF treatments, respectively). The fraction of sexual females was calculated as the number of females with sexual eggs (male and diapausing eggs) divided by the total number of mature individuals (i.e., adults with male, diapausing, or amictic eggs). Non-hybrid populations are represented according to their selection history in the evolution experiment. The response of hybrid populations from HP and LP selection regimes were combined for analysis (See Figure 1, Table 1). For evolved populations we present means \pm 2 standard errors of observed vales (nonhybrid, $n=3$; hybrid, $n=2$ ). For the ancestral population means and $95 \%$ confidence intervals were obtained by bootstrapping the values observed for a subset of seed genotypes (dashed line; Table S2). 


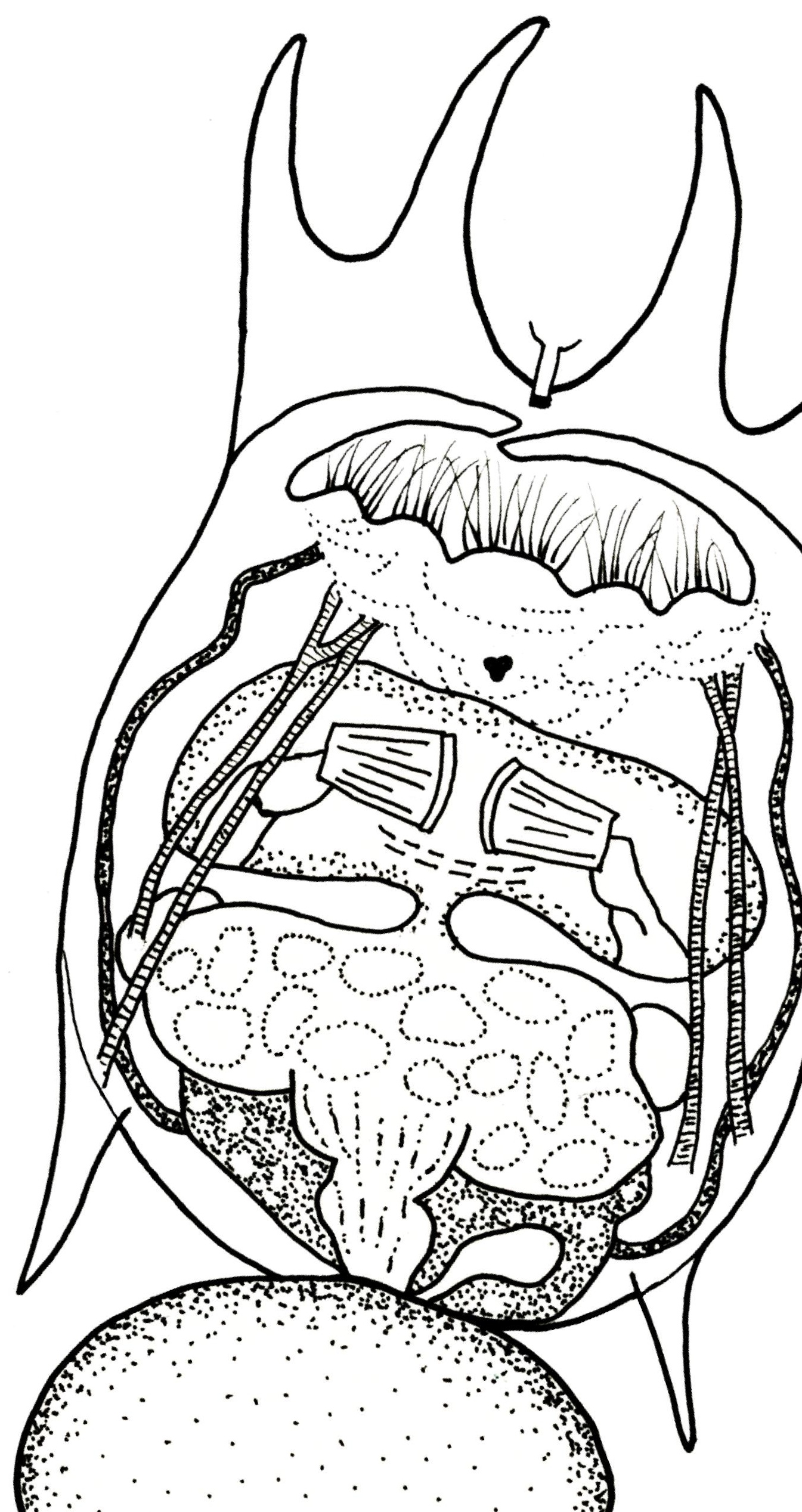




\section{Chapter 4}

Experimental evidence of rapid heritable adaptation in the absence of initial standing genetic variation

Kimberley D. Lemmen, Koen J.F. Verhoeven, \& Steven A.J. Declerck 


\section{Abstract}

1. The success of genetically depauperate populations in the face of environmental change is contrary to the expectation that high genetic diversity is required for rapid adaptation. Alternative pathways such as environmentally induced genetic modifications and non-genetic heritable phenotypes have been proposed mechanisms for heritable adaptation within an ecologically relevant timeframe. However, experimental evidence is currently lacking to establish if, and to what extent, these sources of phenotypic variation can produce a response.

2. To test if adaptation can rapidly occur in the absence of initial standing genetic variation and recombination in small populations, we (i) exposed replicate monoclonal populations of the microzooplankton Brachionus calyciflorus to a culturing regime that selected for phenotypic variants with elevated population growth with either high or low phosphorus food for a period of 55 days and (ii) examined population level response in two fully factorial common gardens at day 15 and 35 of the exposure experiment.

3. We observed rapid (five generations) heritable local adaptation to nutrient limitation, however the capacity for such a response differed between genotypes.

4. Our study finds that although standing genetic variation is considered essential for rapid heritable adaptation, we find that alternative sources of phenotypic variation may also play a role and could aid in the establishment and persistence of low genetic diversity populations.

\section{Keywords}

Brachionus calyciflorus, ecological epigenetics, ecological stoichiometry, intraspecific variation, mutation, non-genetic inheritance, phosphorus, transposable elements 


\section{Introduction}

Environmental fluctuations present a fundamental challenge to organisms as changing conditions may result in a mismatch between the environment and organismal phenotypes. To persist and thrive, populations must have the capacity to modify phenotypes on a timeframe relevant to environmental variation. The ability to adapt has become acutely important during the current period of anthropogenic environmental change in which climate events have become more extreme and frequent (Collins et al. 2013), and habitats are increasingly disturbed (Fischer \& Lindenmayer, 2007; Schweiger et al. 2010; Smith et al. 1999). Adaptive trait shifts within populations are typically considered to occur in two different ways. First, at the individual level, phenotypic plasticity allows organisms to modify their phenotype in response to an environmental trigger (Bradshaw, 1965). Secondly, at the population level, selection may act upon standing genetic variation to shift mean trait values within the population over several generations (Barrett \& Schluter, 2008). Recently, both environmentally induced genetic modifications (Rey et al. 2016) and non-genetic heritable phenotypes (Bonduriansky \& Day, 2018) have been proposed as additional pathways for rapid adaptation. However, experimental evidence is currently lacking to establish if, and to what extent, these sources of phenotypic variation can produce an adaptive heritable response in populations within a timeframe relevant for rapid environmental change (Hu \& Barrett, 2017; Marin et al. 2020; Perez \& Lehner, 2019).

In the last twenty-five years, selection on standing genetic variation has been firmly established as the dominant process through which populations may adapt within ecologically relevant timescales (Barrett \& Schluter, 2008; Bolnick et al. 2011). This process requires high genetic diversity for natural selection to act on. Some populations with low levels of standing genetic variation, nevertheless show a remarkable ability to maintain performance in the face of environmental change (Rollins et al. 2013). Natural processes such as range expansions (Eckert et al. 2008; Excoffier et al. 2009) and the colonization of vacant patches within a metapopulation (De Meester et al. 2002) have the potential to generate low diversity populations. For example, Japanese populations of the cyclical parthenogenic zooplankton Daphnia pulex were founded by only two genotypes following a natural, long-distance dispersal event from North America (So et al. 2015). In addition, anthropogenic change increasingly contributes to the genetic impoverishment of many populations. Habitat destruction (Lino et al. 2019), degradation (Mather et al. 2015) and fragmentation (Schlaepfer et al. 2018) generate small isolated populations vulnerable to drift and genetic erosion. Furthermore, anthropogenic biological invasions frequently give rise to populations with lower genetic diversity than native range populations (Allendorf \& 
Lundquist, 2003). Loss of genetic diversity can be especially pronounced in clonal species where single individuals are able to establish populations. The success of a clonal invader is exemplified by the introduction of a single genotype of $D$. pulex during the stocking of largemouth bass in Lake Naivasha in Kenya during the 1920s (Mergeay et al. 2006). This genotype was able to establish and outcompete native Daphnia species and is now found in water bodies along a $5500 \mathrm{~km}$ continental transect. Similar extremely successful invasion events from a small number of clonal genotypes have also been observed in mollusks (Gomes et al. 2016), plants (Hollingsworth \& Bailey, 2000; Shi et al. 2018) and sexual fish species (Golani et al. 2007).

The success of populations with low standing genetic diversity has sparked great interest in the possible mechanisms that generate adaptive heritable phenotypic variation from a single genotype. While de novo mutations have the potential to drive adaptive responses (e.g., Barrick \& Lenski, 2013; Chan et al. 2010; Hartley et al. 2006; Hoekstra et al. 2006), they have long been considered too infrequent to be relevant on ecological time scales, especially when population sizes are small (Hermisson \& Pennings, 2005). Rapid generation of genetic variation may however be possible through transposable element activity. Recent evidence suggests that novel and stressful conditions can increase the activity of transposable elements, resulting in environmentally induced bursts of genetic variation (Miousse et al. 2015). As some variants may be beneficial, transposable elements have been suggested to be important during adaptation to environmental change (Pimpinelli \& Piacentini, 2020; Rey et al. 2016; Stapley et al. 2015). Heritable phenotypic variation may also be produced by mechanisms other than changes in DNA sequences. Non-genetic inheritance (NGI) allows environmentally induced phenotypes to be passed between generations via the transmission of factors (e.g., nutrients, methylation patterns, and small RNAs) that modify phenotypes (Bonduriansky \& Day, 2018). Given the capacity of NGI to generate heritable phenotypic variation, it has been proposed to play an important role in adaptation (Day \& Bonduriansky, 2011; Jablonka \& Raz, 2009; O'Dea et al. 2016). Sources of de novo phenotypic variation, such as environmentally induced mutations and NGI, are expected to be especially important for adaptation in clonal organisms (Verhoeven \& Preite, 2014) or organisms with low dispersal capabilities (Jablonka \& Raz, 2009; Jeremias et al. 2018). These mechanisms of variation would also be expected to promote adaptation in populations with low genetic diversity (Rapp \& Wendel, 2005; Vogt, 2017) such as invasive species (Marin et al. 2020; Pérez et al. 2006; Prentis et al. 2008).

The capacity to produce environmentally induced heritable variation can be considered an adaptive trait that allows genotypes to persist when conditions 
fluctuate on a temporal scale greater than that of a single generation ( $O^{\prime}$ Dea et al. 2016). An important question is whether there is genetic variation for this capacity within populations, as its extent may determine their evolutionary potential to respond to changes in the frequency and intensity of environmental fluctuation. Previous studies have documented that genotypes may differ in both the magnitude and direction of de novo phenotypic responses to the same stressor (e.g., Asselman et al. 2015; Gillis \& Walsh, 2019; Herman \& Sultan, 2016), although a dedicated effort to document intraspecific variation is thus far largely lacking.

Currently there are very few empirical examples of rapid adaptive responses in plants or animals generated by novel heritable phenotypic variation, despite evidence of the mechanistic potential (Hu \& Barrett, 2017; Marin et al. 2020; Perez \& Lehner, 2019). The goal of this study was to experimentally test if adaptation can occur in the absence of initial standing genetic variation and recombination in small populations within an ecologically relevant time frame. To investigate this question, we conducted a two-part experiment using two genotypes of the microzooplankton Brachionus calyciflorus. In the first part of the experiment (further referred to as the 'exposure experiment'), we exposed replicate monoclonal populations to a culturing regime selecting for phenotypic variants with elevated population growth rates for at least 20 generations with either high (HP) or low (LP) phosphorus food. LP food has a C:P content that deviates strongly from the requirements for growth in $B$. calyciflorus, and thus represents low quality food in comparison to HP food (Sterner \& Hessen, 1994; Zhou et al. 2018). In the second part of the experiment, we performed two full factorial common garden experiments in succession using individuals isolated from the exposure experiment at different moments in time. With these experiments we aimed to evaluate i) if and to what extent an adaptive heritable response has evolved, ii) if clones differ in response strength, and iii) if response strength increases with the duration of exposure to the divergent environmental conditions. We test the hypotheses that an adaptive heritable response occurs within the exposed populations consistent with local adaptation to the food sources; however, genotypes may differ in the magnitude of their response. Additionally, as several generations may be required to elicit an NGI response, or for an adaptive de novo variant to spread through the population, we predict the adaptive response to be greater in the second compared to the first common garden experiment. 


\section{Materials \& Methods}

\section{Model System}

The freshwater microzooplankton $B$. calyciflorus is an excellent metazoan system to study the role of de novo heritable phenotypic variation, because as a cyclical parthenogenetic species it can produce asexual offspring via apomictic parthenogenesis. As such, in asexual populations, recombination can be excluded as a mechanism responsible for the generation of heritable phenotypic variation. When monogonont rotifers reproduce asexually, the oocytes undergo a single maturation with the production of one polar body, and there is no indication for synapsis of homologous chromosomes (Birky \& Gilbert, 1971). Thus, under apomixis, $B$. calyciflorus prevents recombination by inhibiting meiosis I and can asexually produce offspring that are genetically identical to itself. While ameiotic crossovers are possible (Omilian et al. 2006), they are rarely observed on time frames relevant to experimental work in other apomictically reproducing zooplankton (Dukić et al. 2019). B. calyciflorus is also able to reproduce sexually, but this can be avoided by maintaining experimental populations at low densities (Stelzer \& Snell, 2003). When sexual reproduction does occur, it results in the production of diapausing embryos that can easily be distinguished from parthenogenetic eggs (Figure S1). This aspect of $B$. calyciflorus' life history offers the additional benefit that genetically distinct lines can easily be produced and compared. Although previous investigations of de novo heritable variation have taken advantage of apomictic reproduction in other zooplankton species (e.g., Gillis \& Walsh, 2019; Kielland et al. 2017; Walsh et al. 2015), the short generation time of B. calyciflorus (Declerck \& Papakostas, 2017; Stelzer, 2017) provides the added advantage that many generations can be studied over a relatively short period.

\section{Maintenance of Algae and Zooplankton Cultures}

We maintained cultures of the green algae Chlamydomonas reinhardtii as a food source for rotifer cultures in $2 \mathrm{~L}$ chemostats at $23 \pm 1^{\circ} \mathrm{C}$ with a dilution rate of 0.33 day-1 using WC medium (Kilham, Kreeger, Lynn, Goulden, \& Herrera, 1998). By varying medium phosphate concentration and light intensity we created two stoichiometric food quality treatments. High phosphorus algae (HP, molar C:P 102.3 $\pm 1.4 \mathrm{SE}$ ) were cultured in media with $65 \mu \mathrm{mol} \mathrm{L}^{-1} \mathrm{P}$ under $\approx 40 \mu \mathrm{mol}$ quanta $\mathrm{m}$ ${ }^{2} \mathrm{~s}^{-1}$ of continuous light, while low phosphorus algae (LP, molar C:P 690 $10.9 \mathrm{SE}$ ) were cultured in media with $15 \mu \mathrm{mol} \mathrm{L-1} \mathrm{P}$ and under $\approx 120 \mu \mathrm{mol}$ quanta $\mathrm{m}^{-2} \mathrm{~s}^{-1}$ of continuous light. To ensure that phosphorus was not available to the rotifers directly from the media we centrifuged the algae collected from the chemostats and re-suspended the algae pellet in nutrient-free WC media at the desired concentration. Carbon concentration of the suspension was estimated by 
converting biovolume (Multisizer 3 Coulter Counter, Beckman Coulter) to carbon with a previously established calibration curve. After dilution, a vitamin mixture (Kilham et al. 1998) was added to the algae suspension at a concentration of $1 \mathrm{~mL}$ L-1.

For this study we used two genetically distinct clone lines, Clone-7 and Clone-128, belonging to the species $B$. calyciflorus s.s. of the $B$. calyciflorus species complex (Michaloudi et al. 2018). We established clone lines by hatching diapausing eggs that had been produced in the laboratory (Appendix S1). During the two-month period between hatching and initiation of the exposure experiment we maintained clone lines in asexually reproducing stock cultures. We maintained stock cultures at a density of 10 rotifer individuals $/ \mathrm{mL}$ with $\sim 1000 \mu \mathrm{mol} \mathrm{L}{ }^{-1} \mathrm{C}$ algal suspension comprised of nutrient replete C. reinhardtii and nutrient-free WC media (Kilham et al. 1998) at room temperature under a 16:8 (light:dark) light cycle. To keep the genetic identity of the clone line intact we systematically removed all sexually produced eggs daily.

\section{Part One: Exposure Experiment}

For each genotype, we generated five replicate populations for both food quality treatments (HP and LP) by haphazardly allocating ten individuals from stock cultures to wells of tissue culture plates with $8 \mathrm{~mL}$ of the designated algal suspension at a concentration of $1000 \mu \mathrm{mol} \mathrm{L-1} \mathrm{C}$. The entire design of the exposure experiment thus consisted of 20 populations (i.e., 2 clones $x 2$ food qualities $\times 5$ replicates).

During the exposure experiment, we maintained populations in culturing conditions that allowed for density independent growth. To achieve these conditions, we restarted the populations every 24-hours by transferring ten individuals to a new culturing vessel with fresh algal suspension at satiating concentrations ( $1000 \mu \mathrm{mol} \mathrm{L-1} \mathrm{C}$ ). Selection of these individuals was done in a haphazard fashion except we avoided females bearing sexual diapausing embryos. If fewer than ten rotifers were available, we transferred all individuals without diapausing embryos. By transferring a subset of the populations daily, we created conditions that favoured phenotypes with high reproductive output, as offspring of such individuals would be more abundant in the population and thus more likely to be transferred. Additionally, we prevented sexually produced individuals from entering the populations as diapausing embryos require at least 10 hours to be produced, followed by an additional 72 hours to hatch (Stelzer, 2017). Following the daily transfer, we counted the number of remaining individuals in each population to calculate the population growth rate realized during that time interval. 
The exposure experiment lasted 55 days. As time to first reproduction and rate of egg production differ with resource P-content (Zhou et al. 2018), the length of the exposure experiment represents approximately 32 generations in HP conditions and 20 generations in LP conditions (Appendix S2 for details). Throughout the experiment, populations were incubated at $25 \pm 1{ }^{\circ} \mathrm{C}$, in constant darkness and at a rotation rate of $50 \mathrm{rpm}$ to prevent algae from settling. Plate position in the incubator was randomized daily to prevent any possible location effects.

\section{Part Two: Common Garden Experiments}

Using the populations from the exposure experiment, we conducted two common garden experiments to test for a persistent signal of adaptation to stoichiometric food quality, and to determine if these responses changed with the duration of exposure. The first experiment began on day 15, and the second on day 39, of the exposure experiment and lasted for 20 (11 HP generations, 7 LP generations) and 15 days (8 HP generations, 5 LP generations), respectively. For both common garden experiments we cultured individuals from each population from the exposure experiment in both HP and LP algal suspensions and measured population growth rate to determine performance. Each common garden experiment consisted of a fully crossed experimental design (i.e., 2 food quality treatments $\times 2$ clones $\times 2$ exposure histories $\times 5$ exposure populations $=40$ common garden units). Each common garden unit was initiated by allocating ten haphazardly chosen individuals from the respective exposure population. We cultured common garden units in $8 \mathrm{~mL}$ of algal suspension with a concentration of $1000 \mu \mathrm{mol} \mathrm{L}-1 \mathrm{C}$ at $25 \pm 1^{\circ} \mathrm{C}$ and in constant darkness. As in the exposure experiment, at the end of each 24-hour time interval we counted all rotifers and transferred ten of them to new algal suspensions. However, we did not select against females with sexual eggs to measure the true population growth rate (i.e., including the demographic cost of sexual reproduction; Montero-Pau et al. 2014).

\section{Data Analysis}

For both the exposure and common garden experiments, population growth rate was calculated on a daily basis as $\left(\ln \mathrm{N}_{\mathrm{t}}-\ln \mathrm{N}_{0}\right) / \mathrm{t}$, where $\mathrm{N} 0$ and $\mathrm{Nt}$ represent the population size at the start and end of each 24-h period, and $t$ the duration of the period in days. Population growth rates were In-transformed prior to analysis to reduce variance in the residuals between groups.

We wanted to determine if any change in population growth rate had occurred within the populations during the exposure experiment. Furthermore, if a change had occurred, we aimed to detect whether it was immediate (i.e., increased or 
decreased during the first or second generation followed by a saturation of the response) or if the change occurred gradually throughout the experiment. As our populations are composed of multiple individuals, an immediate change would suggest that the majority of individuals were expressing the same phenotype. Such a population level response may indicate the induction of a plastic phenotype in each generation (Sentis et al. 2018), the NGI of an induced phenotype (Richards, 2006), or possibly a combination of both processes. Alternatively, if population growth rate changed gradually it may be due to a tendency of the genotype to accumulate NGI factors across consecutive generations, such as a progressive increase of regulatory small RNAs (Houri-Ze'evi et al. 2016). Alternatively, a gradual change could also reflect the gradual increase in frequency of a successful genetic or epigenetic mutation over successive generations (O'Dea et al. 2016). To distinguish between these two scenarios, for each population from the exposure experiment we constructed both a piecewise (immediate change followed by stability) and linear (gradual change) regression model for population growth over time and identified the model of best fit using the Akaike Information Criterion (i.e., model performance is assumed to differ when $\triangle \mathrm{AIC}>2$; (Burnham \& Anderson, 2004). If the piecewise regression was found to be the best model, we used the Davies test to determine whether there was a significant difference in slopes before and after the breakpoint. As there was no evidence that breakpoint models performed better than linear models, we explored linear trends in growth rate over time using linear mixed effects models (LMM) for each clone by resource quality combination separately. In these four analyzes we treated time as a fixed effect and the population ID from the exposure experiment as a random effect. For the LMM analysis, the first day of the exposure experiment was excluded to avoid any influence from previous culturing conditions.

We tested the effect of exposure history (HP-vs. LP-exposed populations) and its interaction with food quality (HP vs. LP) in the two clones across the two common garden experiments using a LMM. For this analysis we used 'common garden experiment', 'food exposure history', 'common garden diet', and 'clone' as fixed effects. Experimental units of the exposure experiment were specified as a random effect. Population growth rate in this analysis was calculated as the mean of the growth rates of the last ten days of the common garden experiment. Thus, taking into account the difference in growth rates between populations exposed to HP and LP diets (Zhou et al. 2018), we analyzed the average population growth rate for approximately the $6^{\text {th }}-12^{\text {th }}$ generation in the HP treatment, and the $4^{4^{\text {th }}-7^{\text {th }}}$ generation in the LP treatment of the first common garden. In the second common garden, which was five days shorter, we analyzed the average population growth rate for the $3^{\text {rd }} 9^{\text {th }}$ generation in the HP treatment, and the $2^{\text {nd }-6^{\text {th }}}$ generation in the $L P$ treatment. As we are interested in genotype specific fitness response patterns 
concordant with local adaptation, we used a priori contrasts to compare populations with HP- and LP-exposure histories for each genotype in each food treatment across common garden experiments.

All statistical analyses including model selection, a priori contrasts, and post-hoc tests were performed in the R software environment 4.0.2 (R Core Team 2020). We performed LMM with the Ime4 package (Bates et al. 2015) and piecewise regression using the package 'segmented' (Muggeo, 2008). We obtained statistical significances from type II sums of squares using the car package (Fox \& Weisberg 2019) and we used Kenward-Roger degrees of freedom for the LMM. A priori contrasts were performed with the package emmeans (Lenth et al. 2019).

\section{Results}

\section{Part One: Exposure Experiment}

The piecewise regression model performed better than the linear model in only three of the 20 populations from the exposure experiment and in these cases, we found no significant differences between the slope before and after the breakpoint (Table S1). In both HP and LP exposure treatments the population growth rate of Clone-7 increased significantly over the course of the experiment (Figure 1, Table 1 ), by a factor of $46 \%$ and $34 \%$ in the HP and LP treatments, respectively. In contrast, no such increase was found for Clone-128.
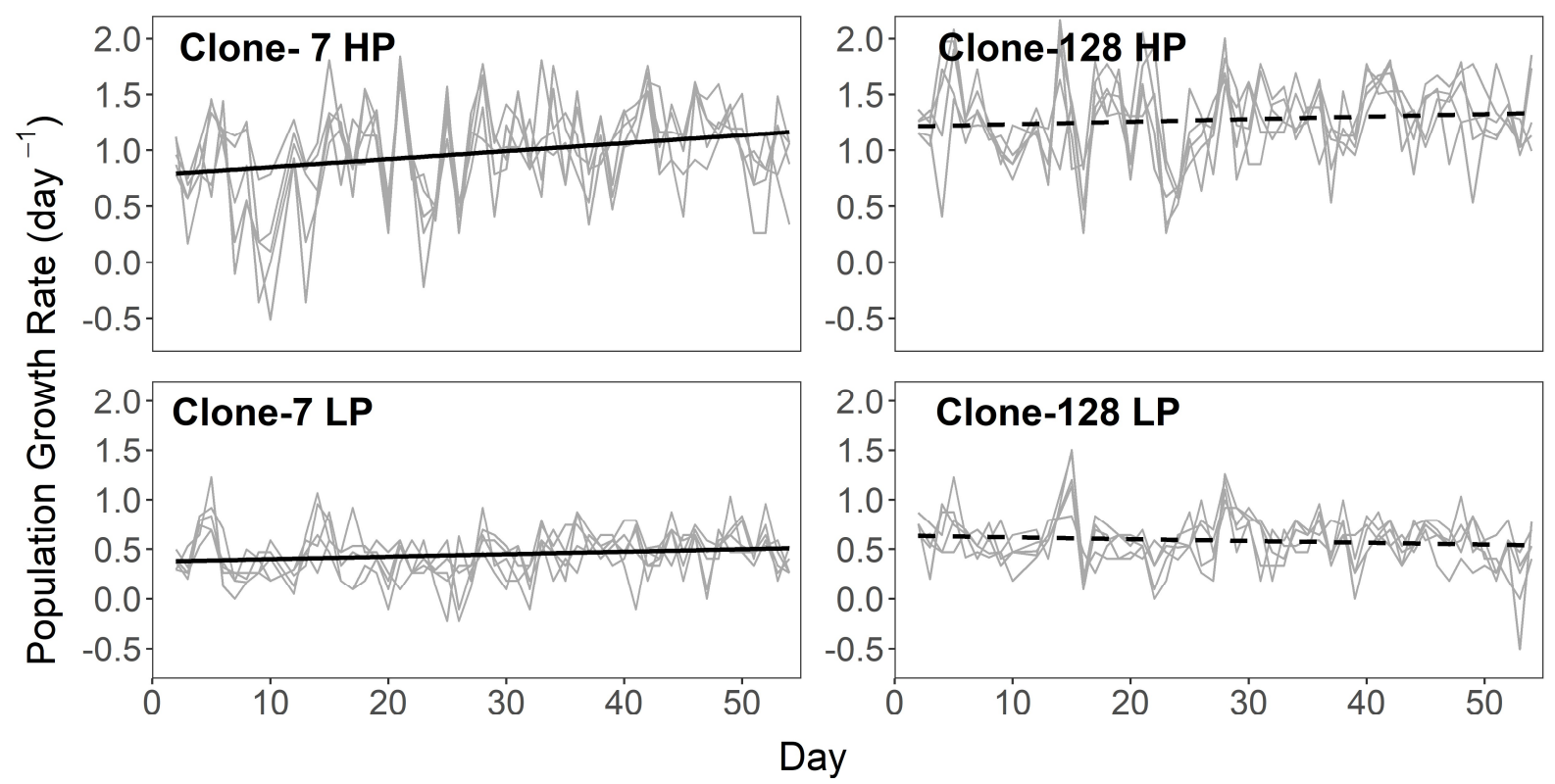

Figure 1. Population growth rate over the course of the exposure experiment for the five replicate populations (grey lines) of Clone-7 and Clone-128 cultured with either a high (HP) or low phosphorus (LP) diet. A solid black line represents a significant change in growth rate over time, non-significant slopes are represented by a dashed black line (Table 1). 
Table 1. Summary of the linear mixed effects models describing population growth rate changes through time for each genotype (Clone-7 or Clone-128) in each treatment (HP or LP) of the exposure experiment (see also Figure 1). The effect of experimental unit was specified as a random effect. Bold p-values are significant.

\begin{tabular}{llccccccc}
\hline \multicolumn{1}{c}{ Clone } & Diet & Slope & SS $^{\mp}$ & MS $^{\mp}$ & Num DF $^{\mp}$ & Dem $^{\text {DF }}$ & F value & $\mathbf{p}^{\mp}$ \\
\hline \hline Clone-7 & HP & $7.12 \mathrm{E}-03$ & 3.065 & 3.065 & 1 & 254.0 & 17.83 & $<0.001$ \\
Clone-7 & LP & $2.48 \mathrm{E}-03$ & 0.374 & 0.374 & 1 & 254.0 & 7.06 & $\mathbf{0 . 0 0 8}$ \\
\hline Clone-128 & HP & $2.25 \mathrm{E}-03$ & 0.307 & 0.307 & 1 & 254.0 & 2.54 & 0.112 \\
Clone-128 & LP & $-1.80 \mathrm{E}-03$ & 0.193 & 0.193 & 1 & $249.0^{+}$ & 3.36 & 0.068 \\
\hline
\end{tabular}

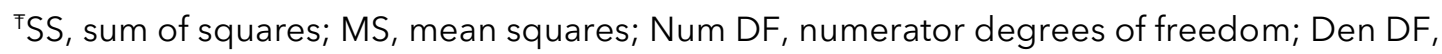
denominator degrees of freedom; p, P-level. ${ }^{+}$data was not collected for all replicate populations of this treatment on day 14 of the experiment due to human error.

\section{Part Two: Common Garden Experiment}

Across common garden experiments and food treatments we observed differences in population growth rate between populations with different exposure history (i.e., HP and LP resource treatments in the exposure experiment) for Clone7, but not Clone-128 (Figure 2, Table 2: $\mathrm{EH}$ * Clone). The effect of exposure history differed between the first and second common garden experiment (Figure 2, Table 2: $\mathrm{EH}$ * Experiment). In both common garden experiments population growth rate was higher in the HP treatment compared to the LP treatment for the two clones. Furthermore, population growth rate of Clone-128 was always greater than Clone-7 (Figure 2, Table 2).

The planned contrasts allowed us to further investigate the effect of exposure history for the two genotypes in each food quality treatment (i.e., HP or LP) across the two common garden experiments. In the low phosphorus common garden treatment Clone-7 populations with an LP exposure history had significantly higher population growth rates than populations with an HP exposure history (a priori contrast, LPexp v. HPexp Clone-7 in LP: $p=0.030$ ). However, we found no significant effect of exposure history on the population growth rate of these populations in the high phosphorus common garden treatment (a priori contrast, LPexp v. HPexp Clone-7 in HP: $p=0.289$ ). For Clone-128 there were no differences between populations with contrasting exposure histories in either the high or low phosphorus common garden treatment (a priori contrast, LPexp v. HPexp Clone128 in HP: $p=0.871$; LPexp v. HPexp Clone-128 in LP: $p=0.871$ ). 


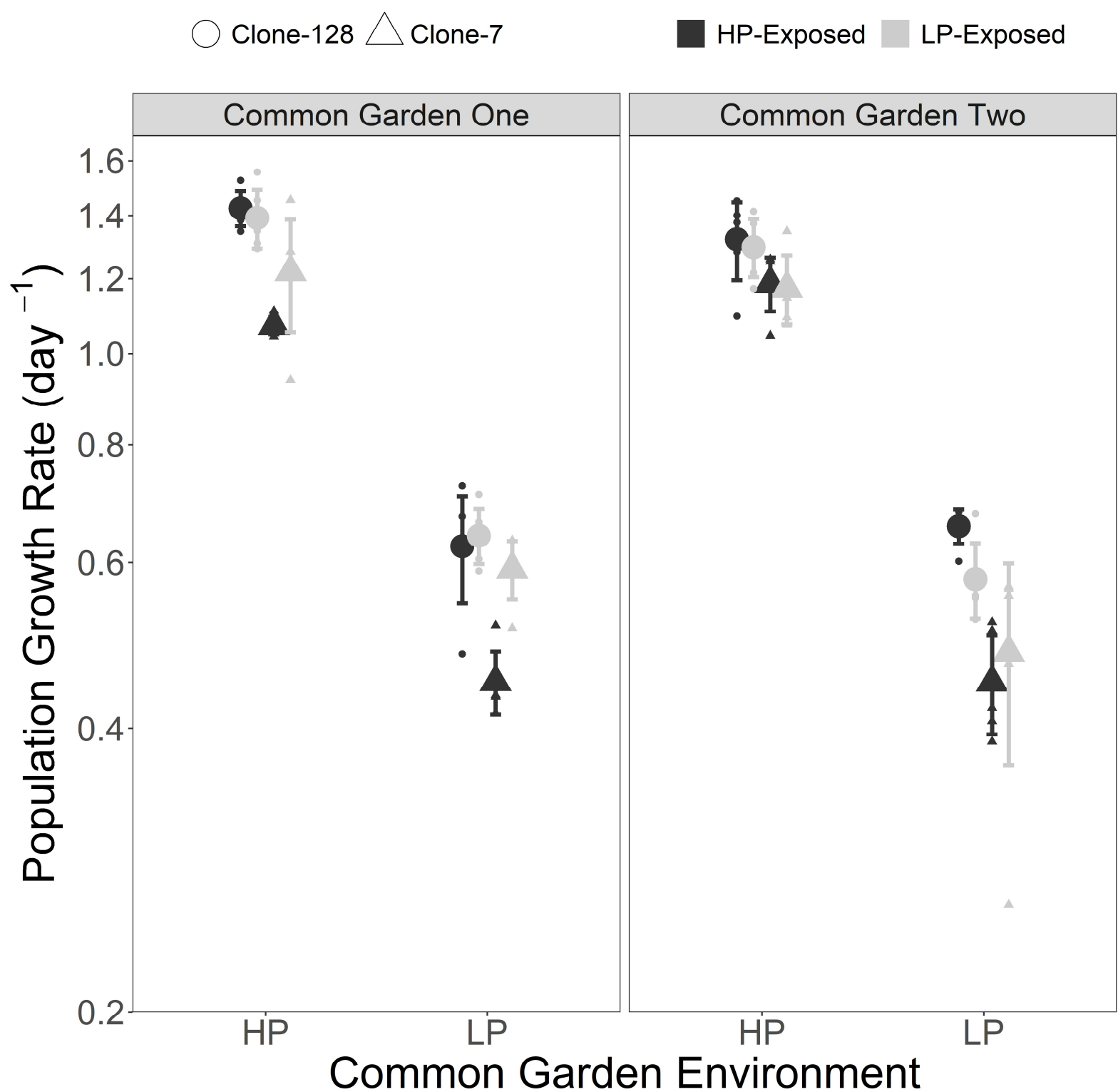

Figure 2. Population growth rate for HP- and LP-exposed populations of Clone-7 and Clone-128 during the first and second common garden experiment with high (HP) and low phosphorus diets (LP). We present the mean population growth rate over the last ten days of the experiment for each experimental unit (small symbols) as well as the grand mean \pm 2 standard errors $(n=5)$ for each clone $\mathrm{x}$ exposure environment combination (large symbols). 
Table 2. Summary of linear mixed effects analyses for the log transformed population growth rate (see also Figure 2). The effects of common garden diet (Diet: HP or LP), exposure history (EH: HPexposed or LP-exposed), genotype (Clone: Clone-7 or Clone-128) and common garden experiment (CG: CG1 or CG2) are presented as the fixed components of the model. The effect of experimental unit was used as a random component of the model. Bold p-values are significant.

\begin{tabular}{lcccccc}
\hline \multicolumn{1}{c}{ Effect } & SS $^{\mp}$ & MS $^{\mp}$ & Num DF $^{\mp}$ & Dem DF $^{\mp}$ & F value & p $^{\mp}$ \\
\hline \hline Diet & $2.58 \mathrm{E}+00$ & $2.58 \mathrm{E}+00$ & 1 & 48 & 870.013 & $<\mathbf{0 . 0 0 1}$ \\
Exposure History (EH) & $4.73 \mathrm{E}-03$ & $4.73 \mathrm{E}-03$ & 1 & 16 & 1.597 & 0.224 \\
Clone & $1.58 \mathrm{E}-01$ & $1.58 \mathrm{E}-01$ & 1 & 16 & 53.294 & $<0.001$ \\
Common Garden & & & & & & \\
Experiment (CG) & $8.41 \mathrm{E}-03$ & $8.41 \mathrm{E}-03$ & 1 & 48 & 2.840 & 0.098 \\
Diet * EH & $1.33 \mathrm{E}-03$ & $1.33 \mathrm{E}-03$ & 1 & 48 & 0.449 & 0.506 \\
Diet * Clone & $8.27 \mathrm{E}-03$ & $8.27 \mathrm{E}-03$ & 1 & 48 & 2.792 & 0.101 \\
EH * Clone & $1.88 \mathrm{E}-02$ & $1.88 \mathrm{E}-02$ & 1 & 16 & 6.362 & $\mathbf{0 . 0 2 3}$ \\
Diet * CG & $2.62 \mathrm{E}-03$ & $2.62 \mathrm{E}-03$ & 1 & 48 & 0.884 & 0.352 \\
EH * CG & $1.61 \mathrm{E}-02$ & $1.61 \mathrm{E}-02$ & 1 & 48 & 5.434 & $\mathbf{0 . 0 2 4}$ \\
Clone * CG & $4.00 \mathrm{E}-05$ & $4.00 \mathrm{E}-05$ & 1 & 48 & 0.013 & 0.910 \\
Diet * EH * Clone & $4.15 \mathrm{E}-03$ & $4.15 \mathrm{E}-03$ & 1 & 48 & 1.402 & 0.242 \\
Diet * EH * CG & $4.38 \mathrm{E}-03$ & $4.38 \mathrm{E}-03$ & 1 & 48 & 1.478 & 0.230 \\
Diet * Clone * CG & $9.89 \mathrm{E}-03$ & $9.89 \mathrm{E}-03$ & 1 & 48 & 3.339 & 0.074 \\
EH * Clone * CG & $2.63 \mathrm{E}-03$ & $2.63 \mathrm{E}-03$ & 1 & 48 & 0.888 & 0.351 \\
Diet * EH * Clone * CG & $3.20 \mathrm{E}-04$ & $3.20 \mathrm{E}-04$ & 1 & 48 & 0.109 & 0.743 \\
\hline
\end{tabular}

FSum Sq, sum of squares; Mean Sq, mean squares; NumDF, numerator degrees of freedom; DenDF, denominator degrees of freedom; P, P-level.

\section{Discussion}

In the absence of initial standing genetic variation, our results demonstrate a rapid (six generations) heritable adaptive response to nutrient limitation by parthenogenetically reproducing zooplankton populations. However, the capacity for a heritable shift in population growth rate differed between the two genotypes studied. Populations of Clone-7 displayed a gradual increase in population growth rate over the course of the exposure experiment consistent with local adaptation, and populations with a LP exposure history outperformed populations with a HP exposure history in the low phosphorus common garden treatment. In contrast, Clone-128 displayed stable population growth rates during the exposure 
experiment and there were no differences between populations with contrasting resource exposure histories in the common garden experiment. Contrary to prior studies, we found no evidence that the length of exposure to the culturing conditions strengthen patterns of adaptation. The findings of this study contribute to a burgeoning set of empirical evidence that the generation of novel heritable variation is a significant contributor to adaptation to environmental change at short timescales. This serves to further our understanding of how populations successfully establish and persist in changing environments despite low or no genetic diversity.

\section{Rapid heritable adaptation in response to exposure to low phosphorus resources}

This study provides empirical evidence for rapid heritable adaptation in a monoclonal population, challenging the conventional perspective that initial standing genetic variation is necessary for such adaptation (Barrett \& Schluter, 2008). In the low phosphorus common garden treatment, Clone-7 populations with an LP exposure history had higher population growth rates than populations with an HP exposure history. This pattern is consistent with the "home vs. away" criterion for local adaptation (Kawecki \& Ebert, 2004). Local adaptive responses in the absence of genetic variation are generally attributed to processes such as intragenerational plasticity and anticipatory maternal effects (J. Marshall \& Uller, 2007). However, we can exclude both mechanisms respectively as i) differences in growth rate between populations with contrasting exposure histories lasted longer than four generations in the common garden low phosphorus treatment, and ii) the maternal effect transmitted from low phosphorus mothers is known from previous research to be in the opposite direction from what we observed (Zhou \& Declerck, 2020). A second striking result of this study that points at rapid adaptation, is the increase in population growth rate that occurred in Clone-7 populations over the course of the exposure experiment in both diet treatments. This response suggests gradual adaption to the culturing conditions, and contrasts with rapid phenotypic changes generated by both intra-generational and anticipatory maternal effects in response to stressful environments.

Direct evidence of multigenerational exposure to a stressful environment producing heritable adaptation within initially genetically uniform populations is currently limited. In response to artificial disruptive selection during four or more generations, several studies have observed significant phenotypic differentiation among parthenogenetic lines of aphids or water fleas (Daphnia) that originated from a single foundress (Andrade \& Roitberg, 1995; Bunting \& Van Emden, 1980; Gorokhova et al. 2002; Wilhoit \& Mittler, 1991). So far, using a single clone of the apomictically reproducing pea aphid Acyrthosiphon pisum, Sentis et al. (2018) are 
the only authors that have demonstrated heritable adaptation in response to natural selection. Our study is the first that provides support for their findings by demonstrating rapid heritable adaptation without initial genetic variation in a nonarthropod system. The results of our study thus indicate that the production of environmentally induced heritable phenotypes may be important for adaptation in a wide range of organisms. Furthermore, we observed a heritable adaptive response in less than half the number of generations than in previous studies that imposed selection not by artificial but by natural selection (Sentis et al. 2018). It is likely that such rapid phenotypic change in response to varying environmental conditions could have wide-ranging consequences on the eco-evolutionary dynamics of a system.

The underlying mechanism of the adaptation observed in this study is unknown. Given that the observed phenotypic differences developed very rapidly, and in multiple replicate populations, it is unlikely that spontaneous SNP mutations are responsible for the observed effects (Hermisson \& Pennings, 2005). One candidate mechanism that may have produce the observed adaptive response is an environmentally induced NGI factor that is transmitted for multiple generations even after the removal of the inducing environment. Transgenerationally stable epimutations or small RNAs have this capacity in some systems (e.g., Rechavi et al. 2014). Alternatively, a mechanism that generates rapid genetic variation upon stress exposure, such as transposable element proliferation, could be responsible. A speculative explanation for the observed gradual linear increase in growth rate of Clone-7 in both exposure treatments is that a non-genetic adaptive factor (e.g., small RNA) may have gradually accumulated due to consecutive generations of stressor exposure and resulted in a gradual increase in population growth rate. While multigenerational exposure to stressful environments has been observed to prevent the degradation of non-genetic factors (Houri-Ze'evi et al. 2016), it is unclear if mechanisms exist that would allow for accumulation. Further investigations are required to elucidate the molecular mechanism responsible for the observed adaptive response.

\section{Effect of exposure histories in HP common garden treatment}

Traits that enable adaptation to one environment may come with costs in a different environment (Levins, 1968). However, we did not observe any difference in the relative performance of Clone-7 populations with contrasting exposure histories in the high phosphorus common garden treatment. This may indicate that traits favoured in LP conditions have low physiological costs. Alternatively, the benign nature of the HP common garden environment may have masked subtle trade-offs. Intriguingly, a similar pattern in performance has been observed in several other studies that have used common garden experiments to compare the 
fitness of LP- and HP-adapted populations (Declerck et al. 2015; Frisch et al. 2014; Lemmen et al. 2020). The lack of difference may also be attributed to the fact that generation times of populations fed with phosphorus rich food are considerably shorter than with phosphorus poor food (Zhou et al. 2018), and that non-genetic phenotype modifications tend to degrade across generations after the removal of the environmental trigger (Herman et al. 2014; Houri-Ze'evi \& Rechavi, 2017). Thus, more generations had passed in the high than in the low phosphorus treatments when the monitoring of population growth rates in the common garden experiments started. If the phenotype of populations cultured with LP resources during the exposure experiment was due to NGl, it may have no longer been expressed in the period during which population growth rates were assessed during the common garden. The ambiguity regarding why populations with contrasting exposure histories differ in the low but not the high phosphorus common garden treatment highlights the need for a better mechanistic understanding of the observed adaptation.

\section{Clonal Differences}

The two clones used in this study consistently displayed different responses in both parts of the experiment. Previous work has shown that in a variety of organisms, genotypes differ in both their rates of DNA mutation (Ho et al. 2019; Ness et al. 2015) as well as in their ability to produce non-genetic heritable phenotypes (Leung et al. 2016; Menezes et al. 2018; Seudre et al. 2020). As such, we expected to observe differences between clones, however the magnitude (Clone-128's non-response compared to Clone-7's heritable 26\% increase in population growth rate) was larger than anticipated. The contrasting responses between clones implies a genetic basis for the capacity to produce heritable phenotypes from a single genome suggesting that such a trait could thus be subject to selection. This variation may reflect differences in the environmental stability of their source populations. Within-generation plasticity has been shown to be favoured when environmental change occurs either within, or between, adjacent generations (Botero et al. 2015). In contrast, intergenerational NGI of environmentally induced phenotypes is predicted to be favoured in environments that remain stable for several generations but fluctuate over longer periods, so that parental conditions accurately predict those experienced by the offspring (Herman et al. 2014; Jablonka \& Raz, 2009). This prediction was supported by Walsh et al. (2016) who found that in response to predation cues Daphnia from lakes with stable predation regimes displayed a small within generation shift in phenotype but a large intergenerational response. In contrast, Daphnia from lakes where predation varied within growing seasons displayed the opposite response. While environments that theoretically favour NGl appear to be common (Colicchio \& Herman, 2020), empirical evidence connecting the temporal dynamics of 
environmental conditions to variation in the capacity of NGl in either lab or natural populations is limited. In our study, the two clones originated from different populations, and this may potentially have caused their contrasting responses to the same environmental stressor.

\section{Decrease in performance of LP-exposed populations}

Differences in population growth rates between populations with contrasting exposure histories did not increase with the number of exposed generations (i.e., from the first to the second common garden). This was contrary to our expectations, as a previous study with genetically uniform populations demonstrated a positive relationship between the number of generations exposed to a stressful environment and the magnitude of the stress-induced phenotypic response (Sentis et al. 2018). Instead, we observed that from the first to the second common garden, differences in performance decreased between populations from the exposure experiment provided HP compared to LP resources. Currently, we are unable to explain why the effect of exposure history changed with the increasing number of generations. However, this interaction between exposure history and common garden does not impact the main findings of this study, as for each clone the relationship between contrasting exposure histories in the two food treatments remains consistent across common garden experiments.

\section{Ecological relevance}

Low genetic diversity within populations frequently occurs and can be the result of both natural (Haileselasie et al. 2018; Louette et al. 2007; So et al. 2015) and anthropogenic processes (Roman \& Darling, 2007; Schlaepfer et al. 2018). However, the long-term success of these populations is not well understood, given the potential for mismatch between phenotypes and the environment (Pérez et al. 2006). Our observation of rapid, heritable adaptation in the absence of initial genetic variation, may provide an explanation of how low-diversity populations may expand and persist in a variable environment. For example, in small genetically isolated populations, the production of heritable phenotypes may allow for persistence by enabling populations to track environmental changes in a process akin to evolutionary rescue (O'Dea et al. 2016). Such mechanisms may be particularly important in asexually reproducing organisms with the capacity for long distance dispersal, as they are likely to encounter environments that differ from where they originate. In such circumstances, the ability to produce offspring with a novel, potentially adaptive heritable phenotype would provide long-term fitness benefits during the colonization of empty patches due to priority effects (De Meester et al. 2002). These mechanisms may also play a role in the successful establishment of clonal invasive species (Gomes et al. 2016; Shi et al. 2018). 
However, the benefits of environmentally induced heritable phenotypes are not restricted to clonal organisms, and they have also been speculated to enhance the adaptive capacity of sexually reproducing species (Rollins, Richardson, \& Shine, 2015). Numerous field studies have found correlations between non-genetic variation and environmental divergence in both invasive (Schrey et al. 2012; Sheldon et al. 2018) and natural populations (Wogan et al. 2020). It has yet to be determined if such environmentally driven non-genetic differentiation is heritable, however these studies hint at a possible role of NGl in adaptation and warrant further investigation. Given the unprecedented rate and magnitude of current environmental change, it is important that we understand all possible mechanisms that organisms may use to persist and adapt. We believe the production of heritable phenotypes in response to environmental conditions may play an underappreciated adaptive role, especially in populations with low genetic diversity.

Predicting the establishment success of potentially invasive populations is of great ecological and economic importance in an increasingly interconnected world (Kolar \& Lodge, 2001). Genetically depauperate populations have resulted in both failed (e.g., Hairston et al. 1999) and incredibly successful invasions (e.g., Mergeay et al. 2006). However, it is generally unknown why some invasions succeed while others do not (Zenni \& Nuñez, 2013). Our study demonstrates differences in the capacity of genotypes to produce environmentally induced variation. Such differences may contribute to the establishment success of populations, and accounting for this trait may improve our ability to predict the invasiveness of different native populations. For example, populations that display the capacity to produce a diversity of heritable phenotypes in response to an environmental trigger could be classified as "high invasion risk", and stricter measures for decontamination may be put in place for transport vectors originating from these populations (Fournier et al. 2019). From a conservation perspective, knowledge of the environmental conditions that favour genotypes that can rapidly adapt to environmental change may help prioritize what populations to protect given limited resources. For example, preserving existing standing genetic variation would be of high priority in populations that are predicted to have a limited capacity for environmentally triggered adaptive phenotypes. Further empirical research is needed to understand the conditions under which the capacity for spontaneous phenotype production may be adaptive. This work could help identify not only potentially highly invasive populations but also characterize the extinction risk of populations in the face of environmental change. 


\section{Conclusions}

Despite frequent speculation of the ecological and evolutionary importance of mechanisms for generating heritable phenotypic variation (Day \& Bonduriansky, 2011; Jablonka \& Raz, 2009; O'Dea et al. 2016) there is limited empirical evidence of adaptive responses, especially in animal populations (Hu \& Barrett, 2017; Perez $\&$ Lehner, 2019). Our study provides a clear example of rapid heritable local adaptation in response to exposure to a stressful environment based on de novo heritable variation in an animal population. As seen in previous studies (Menezes et al. 2018), the capacity to produce heritable phenotypic variation was genotype dependent, and furthermore, was only observed in populations with a low phosphorus exposure history. A better understanding of the mechanisms responsible for producing phenotypes is needed to provide insight into the observed genotypic differences, and their relevance in ecological processes. Thus far, all experimental investigations of adaptation in the absence of genetic variation have been performed either in microbes or in short-lived invertebrates (i.e., $D$. pulex, A. pisum, and B. calyciflorus). To determine whether the adaptive response observed in this study is pertinent in other taxa, further investigations are needed with a wider diversity of organisms. Although standing genetic variation is considered essential for rapid heritable adaptation, we find that alternative sources of phenotypic variation may also play a role and could aid in the establishment and persistence of low diversity populations.

\section{Acknowledgements}

This work was supported by the Division for Earth and Life Sciences (ALW) from the Netherlands Organization for Scientific Research (NWO) and a NIOO-KNAW strategic fund. We wish to thank Laurens Verhage for laboratory assistance throughout the study, Wei Zhang for allowing us to make use of his laboratory egg banks from which we extracted our focal clones and Libin Zhou for supplementary laboratory assistance. We also thank Cam Hudson for constructive comments on the manuscript. 


\section{Supplementary Materials}

\section{Appendix S1: Additional Methodological Information}

We established the two clone lines used in this study by hatching diapausing embryos from "egg banks" that had previously been established by the research group. To create the lab banks, we first isolated diapausing embryos from sediment collected in the field by applying the sugar flotation method described by Gómez and Carvalho (2000). We placed diapausing embryos in petri dishes with WC medium (Guillard, 1975) and exposed them to constant light at room temperature to stimulate hatching. We checked the dishes at $12 \mathrm{~h}$ intervals. Hatched females that appeared to be Brachionus calyciflorus were transferred to individual wells of 24-well plates filled with $1 \mathrm{~mL}$ of chemostat cultured Chlamydomonas reinhardtii $(1000 \mu \mathrm{mol} \mathrm{C} \mathrm{L-1}$, molar C:P ratio of approximately 150:1) resuspended in WC medium. Each individual collected represented a single genotype and was thus cultured separately. Following the production of offspring we used two individuals to confirm the species ID of each genotype using RFLP analysis (Papakostas et al. 2016), any lines that were not B. calyciflorus were discarded. We created the artificial egg bank by combining individuals from 15 randomly selected genotypes in equal proportion at a high density to promote sexual reproduction. Every 48 hours we separated the diapausing embryos that had been released by the females from the live individuals. For long term storage we used vacuum filtration to collect the diapausing eggs on GFF filters which were then kept wrapped in foil in the dark at $4^{\circ} \mathrm{C}$. To hatch the clones used in this experiment we placed half a filter in WC medium in a petri dish and followed the previous outlined methodology to stimulate hatching and collect newly emerged individuals. For this experiment Clone-7 originated from 'Pond 7' $\left(51.854065^{\circ} \mathrm{N}\right.$ $5.893175^{\circ} \mathrm{E}$ ) and Clone-128 from 'Pond 128' (52.640324' N 4.730287 $\left.\mathrm{E}\right)$. Both ponds are in The Netherlands and are not hydrologically connected.

\section{Appendix S2 - Description of generation estimation}

To estimate the number of generations that passed over a given time period in the high and low phosphorus food quality treatments in both the exposure and common garden experiments we used a simple simulation. We first searched the literature to determine i) the average lifespan of an individual [153 hours, (Kauler \& Enesco 2011)] and ii) the average number of lifetime reproductive events [14, (Kauler \& Enesco 2011)]. For each food quality treatment we then determined iii) the time at which the first reproductive event occurred [HPF: 29 hours, LPF: 42 hours; (Zhou et al. 2018)] and iv) the rate at which reproduction events occurred [HPF: 0.375 individuals hour ${ }^{-1}$, LPF: 0.133 individuals hour-1; (Zhou et al. 2018)]. Using this life history information, we performed a simulation that mirrored the 
design of the exposure experiment (see steps below). We repeated this process 10,000 times and the mean of those values is reported in the main text of the study.

1. Generate a population of 10 individuals, designated as generation 0 , with ages randomly drawn from a uniform distribution of 0 to 153 hours.

2. Simulated population growth based on the age of the individuals and the reproductive schedule for each food quality treatment (e.g., In HPF individuals older than 29 hours and less than 68 hours old would produce a single offspring every 3 hours and disappear from the population once they reached an age of 153 hours). All the offspring from an individual were part of the same generation and were classified as generation $P+1$, with $P$ being the generation designation of the parent.

3. After 24 hours, we recorded the generation designation for all individuals within the population and then randomly subsampled 10 individuals to restart the population as in the experimental design and repeated step 2. This population growth followed by restarted was repeated 55 times to match the length of the exposure experiment.

4. At the end of the simulation we used the daily record of generations present record in step 3 to calculate the mean number of generations that passed since the initiation of the experiment for the day of interest (e.g., Day 5, 10, 15,20 and 55) 


\section{Supplementary Tables}

Table S1. Comparison of linear and piecewise regression models performed for each of the experimental units in the exposure experiment using AIC. The symbol * indicates experimental units for which the trend of population growth through time is better described by a piecewise than a linear regression model according to AIC. Davies $p$ : significance of difference between the two slopes of the piecewise regression model. HP = high phosphorus, LP = low phosphorus.

\begin{tabular}{cccccccc}
\hline Clone & $\begin{array}{c}\text { Selection } \\
\text { Diet }\end{array}$ & Replicate & $\begin{array}{c}\text { Linear } \\
\text { AIC }\end{array}$ & $\begin{array}{c}\text { Piecewise } \\
\text { AIC }\end{array}$ & A AIC & $\begin{array}{c}\text { Piecewise } \\
\text { Best } \\
\text { Model }\end{array}$ & $\begin{array}{c}\text { Davies } \\
\mathbf{p}\end{array}$ \\
\hline \hline 128 & HP & 1 & 28.21 & 27.08 & 1.13 & & \\
128 & HP & 2 & 43.93 & 44.36 & -0.43 & & \\
128 & HP & 3 & 52.05 & 51.85 & 0.2 & & \\
128 & HP & 4 & 56.43 & 58.75 & -2.32 & & \\
128 & HP & 5 & 34.97 & 35.36 & -0.39 & & \\
\hline 128 & LP & 1 & 10.99 & 11.99 & -1 & & \\
128 & LP & 2 & -8.93 & -6.15 & -2.78 & & \\
128 & LP & 3 & 9.22 & 5.25 & 3.97 & $*$ & 0.401 \\
128 & LP & 4 & -0.45 & 2.53 & -2.98 & & \\
128 & LP & 5 & 7.45 & -1.16 & 8.61 & $*$ & 0.818 \\
\hline 7 & HP & 1 & 42.14 & 44.96 & -2.82 & & \\
7 & HP & 2 & 63.14 & 61.11 & 2.03 & $*$ & 0.919 \\
7 & HP & 3 & 45.37 & 46.82 & -1.45 & & \\
7 & HP & 4 & 80.72 & 80.83 & -0.11 & & \\
7 & HP & 5 & 57.74 & 58.54 & -0.8 & & \\
\hline 7 & LP & 1 & 13.28 & 16.49 & -3.21 & & \\
7 & LP & 2 & -24.81 & -23.96 & -0.85 & & \\
7 & LP & 3 & -11.43 & -9.76 & -1.67 & & \\
7 & LP & 4 & 10.12 & 9.4 & 0.72 & & \\
7 & LP & 5 & -2.37 & -4.02 & 1.65 & & \\
\hline & & & & & & & \\
\hline
\end{tabular}




\section{Supplementary Figure}
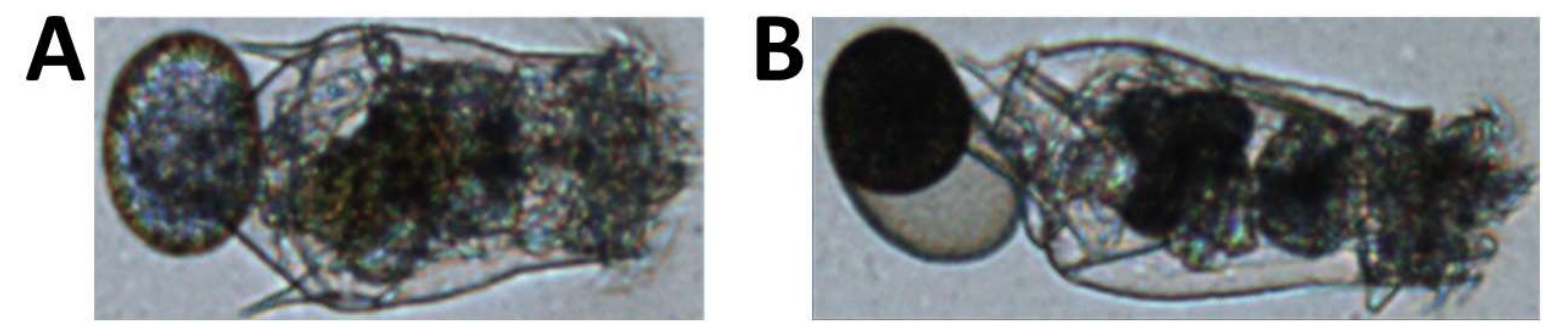

Figure S1. Brachionus calyciforus female with a (A) parthenogenetic egg and (B) diapausing embryo. Parthenogenetic eggs appear as a prolate ellipsoid that has a consistent opacity, whereas diapausing embryos are kidney-shaped and consist of an opaque and transparent pole. In this experiment females have been observed to carry up to three parthenogenetic eggs at a time, but only carry a single diapausing embryo. 


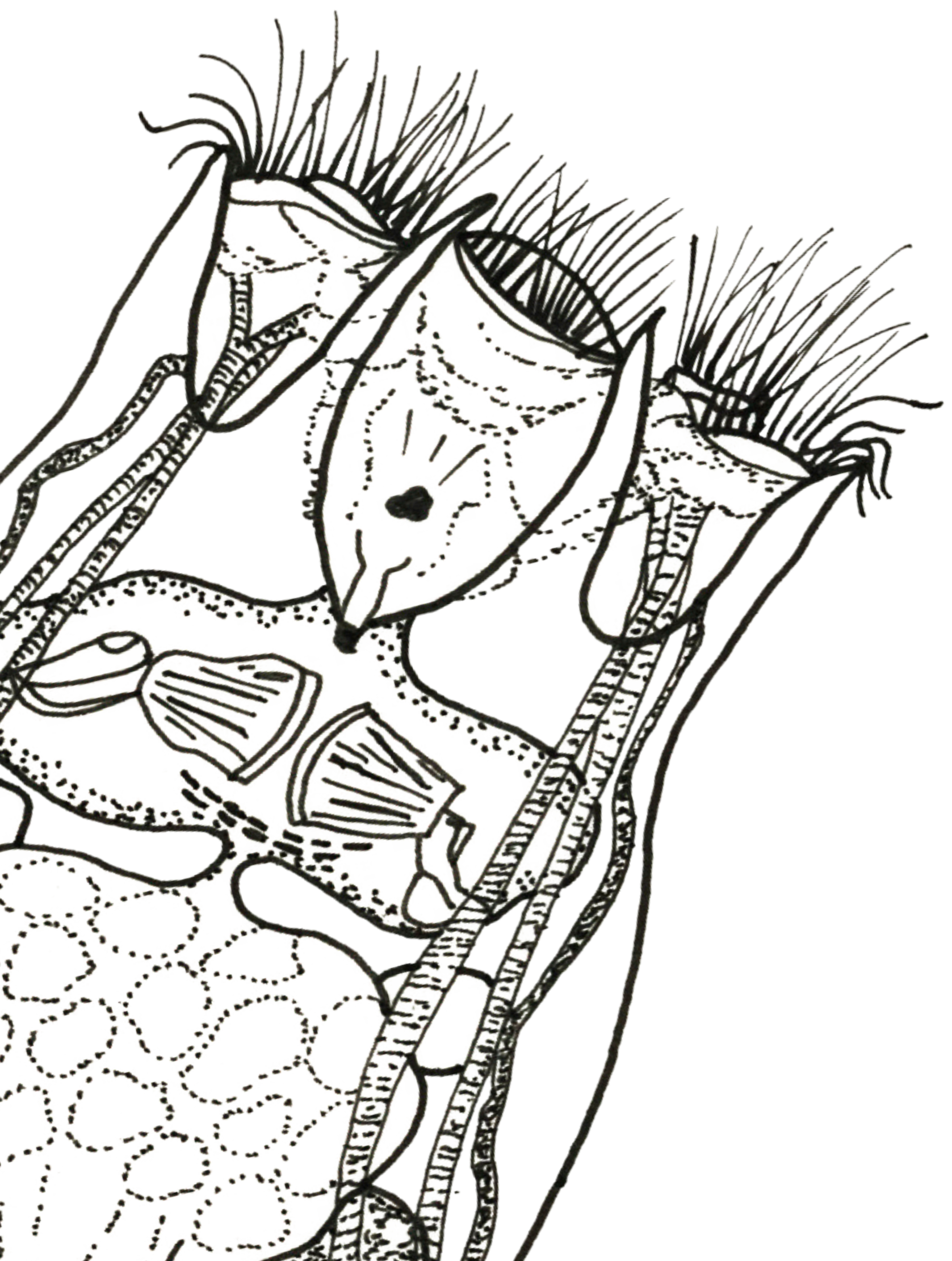




\section{Chapter 5}

Direct and indirect effects of phosphorus limitation on the functional response of the microzooplankton consumer Brachionus calyciflorus

Kimberley D. Lemmen \& Steven A.J. Declerck 


\section{Abstract}

Resource elemental limitation impacts consumer ingestion rates which can have important consequences for trophic interactions. However, the effects of stoichiometric imbalance have primarily been quantified at high resource densities and there is a dearth of investigations into its effect across an ecologically relevant range. Additionally, it is unclear if consumers modify ingestion by directly assessing resource elemental content or whether they rely on other properties that are an indirect result of elemental limitation. A consumer's functional response describes the change in consumption rate that occurs in response to changes in resource density and provides a framework to describe the strength and stability of trophic interactions. To investigate the effect of resource elemental limitation on a herbivore consumer's functional response, and the cues used to modify this behaviour, we conducted ingestion trials across a range of resource densities with the microzooplankton consumer Brachionus calyciflorus and the motile green algae Chlamydomonas reinhardtii as a resource. We used algae with both high (HP) and low phosphorus (LP) content, as well as a third resource quality created by supplementing $L P$-algae with inorganic $P(L P+P)$. The $L P+P$ treatment increased the $P$-content of the algae but retained the other biochemical properties associated with growth in P-limited conditions, allowing for us to disentangle the direct and indirect effects of P-limitation. We observed that herbivore consumers exhibit compensatory feeding at high but not low food densities in response to resource $\mathrm{P}$-limitation, and that modifications in ingestion rate are not directly mediated by the resource's $P$ content, but rather indirectly by other properties associated with algal P-limitation. These results suggest that a decrease in resource quality may increase herbivore top-down control in productive systems and highlights the gaps in knowledge regarding the mechanisms regulating ingestion.

\section{Keywords}

stoichiometry, P-limitation, Holling Type III functional response, rotifer, consumerresource 


\section{Introduction}

Anthropogenic activities have significantly impacted global nutrient cycling, altering the availability of life-essential macroelements such as carbon (C), nitrogen (N), and phosphorus (P) (Stockner et al. 2000; Elser et al. 2009; Smith \& Schindler 2009). The elemental composition of autotrophs, which is more flexible than heterotrophs, often reflects such changes in environmental nutrient supply (Sterner \& Elser 2002). As the relative abundance of elements within an organism (i.e., organismal stoichiometry) changes in response to environmental conditions, so does its quality as a food resource for consumers (van de Waal et al. 2010). Autotrophs with a low C:nutrient are generally considered high-quality resources (Sterner \& Hessen 1994; Urabe et al. 2003) but see (Zhou \& Declerck 2019), as their stoichiometry more closely matches the elemental needs of consumers. However, studies predict that the combined effects of increasing atmospheric $\mathrm{CO}_{2}$ concentrations, climate warming, and reductions in anthropogenic nutrient inputs will increase autotrophs' $\mathrm{C}$ content relative to other nutrients (Verschoor et al. 2013; Verspagen et al. 2014; Verbeek et al. 2018). As a result, consumers may have to cope with deteriorating food quality in the coming decades. To compensate for limiting nutrients, consumers may up- or down-regulate ingestion rates (Ott et al. 2012; Jochum et al. 2017), affecting the strength of top-down control in the ecosystem (Hall 2009; Declerck et al. 2015). Though resource stoichiometry is important for herbivore ingestion rates (Hillebrand et al. 2009), there is a dearth of experiments that investigate its effect across a wide range of resource densities. A more complete understanding of the effect of autotroph stoichiometry on herbivore ingestion is needed to anticipate the impacts of changes in elemental availability on trophic interactions.

The functional response of an organism is a critical behavioural feature that determines the strength and stability of its interaction with its resources (Murdoch \& Oaten 1975; Uszko et al. 2015). Holling (1959) developed the first functional response model describing the change in an individual predator's consumption rate in response to changes in prey density. Holling categorized functional responses into three main types: linear (type I - which are rare (Jeschke et al. 2004) and not discussed further), hyperbolic (type II), and sigmoid (type III). Consumers with a type II response search for prey at a constant rate at all densities. In contrast, consumers' search rate with a type III response increases with prey density, an adaptive response to reduce the cost of foraging at low prey densities (Abrams 1982). It is important to distinguish between type II and III responses as they have contrasting implications for the stability of consumer-resource dynamics. Type II responses often destabilize predator-prey dynamics as prey suffer higher mortality rates at low density because the consumer search rate is density-independent. Thus for a specialist predator, most, and in extreme cases all, prey are eaten at low 
densities, increasing the likelihood of prey extinction (Oaten \& Murdoch 1975; Taylor \& Collie 2003; Gascoigne \& Lipcius 2004; Kramer \& Drake 2010). In contrast, with type III responses, prey mortality is low at low densities. Type III responses stabilize consumer-resource dynamics since prey experience a "mortality refuge" when densities fall below a certain level (Murdoch \& Oaten 1975). However, the relationship between functional response type and stability is not always straightforward (Uszko et al. 2015). Categorizing the functional response "type" with certainty requires high-quality consumption data at low resource densities, which past experimental designs have failed to include (Sarnelle \& Wilson 2008; Uszko et al. 2020). Although type II responses dominate historical literature (Jeschke et al. 2004), type III responses are increasingly being reported, especially in zooplankton (Fussmann et al. 2005; Sarnelle \& Wilson 2008; Seifert et al. 2014; Uszko et al. 2015).

Although type II and III responses were initially defined as two separate models, (Real 1977) developed a generalized functional response equation that allows for a gradual transition between the two different types of consumer behaviour (Equation 1).

$$
F(N)=\frac{b N^{1+q}}{1+b h N^{1+q}}
$$

Where the per capita feeding rate $F$, depends on the number of prey in the environment $N$. Three key parameters describe the shape and slope of the generalized functional response. First, the space clearance constant $b$, which describes how quickly a consumer clears a given resource space and primarily controls the rate at which consumption increases at low densities. Second, the handling time $h$, describes the time investment per resource consumed and encompasses multiple biological processes, including attacking, eating, and digesting (Jeschke et al. 2002). Handling time primarily controls the consumption rate at saturating densities (i.e., low $h$ means the consumers eat more resources in a given period, and the functional response saturates at a higher level). Third, the space clearance rate exponent $q$, defines the shape of the functional response. For a hyperbolic type II response $q=0$, any value significantly greater than 0 is considered a type III response, and as $q$ increases, the shape of the curve becomes more sigmoidal. The study of variation in each of these parameters separately has provided a more mechanistic understanding of consumer foraging responses to variables such as consumer body size and temperature (Englund et al. 2011; Rall et al. 2012; Daugaard et al. 2019). For example, increases in handling time will result in a lower maximum ingestion rate, weakening top-down control of the system. In contrast, increases in the search rate constant will increase prey consumption at low prey densities and strengthen top-down control. Increases in q stabilize predator-prey interactions as consumer responses transition from type II to III. 
Elemental limitation of resources impacts multiple organismal traits (e.g., assimilation, respiration, excretion), however the effects of stoichiometric imbalance on the parameters of an herbivore's functional response remain unclear. Increased consumption (i.e., "compensatory feeding", [Lincoln et al. 1986]) is commonly observed in response to nutrient limitation when resources abundant (e.g., Plath \& Boersma 2001; Fink \& Von Elert 2006; Flores et al. 2014; Zhou \& Declerck 2019). However, as most studies explicitly investigating the effects of resource stoichiometry typically only have one or two food quantity levels, it is unknown whether and to what extent compensatory feeding occurs across the range of naturally occurring food concentrations (Dick et al. 2014). Of particular interest is how resource quality affects consumption at low food densities (i.e., the space clearance constant). Compensatory feeding is predicted to occur once energy (Carbon) is in excess and can be directed toward increasing limiting nutrient uptake (e.g., increasing movement to increase encounters) (Suzuki-Ohno et al. 2012). However, empirical evidence is currently inconclusive. Some ingestion trials have observed no compensatory feeding (Fink \& Von Elert 2006) whereas others show increased consumption of low-quality food (Ott et al. 2012; Flores et al. 2014). Accurately documenting ingestion rates at low resource densities is particularly important in understanding the effects of alterations in elemental availability because worsening food quality is often associated with lower resource biomass (Jeppesen et al. 2005; Hartwich et al. 2012).

Although resource stoichiometry impacts ingestion rates, it is unclear what cues consumers use to modify their intake and how quickly organisms can respond to changes in food quality. Autotrophs grown in low $\mathrm{P}$ conditions are both deficient in $P$ and biochemically distinct from those that experience P-replete growth (Ravet \& Brett 2006). Algae grown in a low compared to high $P$ environment have thickened cell walls (Tillberg et al. 1984) and reduced fatty acid (EPA) (Müller-Navarra 1995; Boersma 2000) and sterol content (Piepho et al. 2010). It is currently unknown if consumers modify their feeding behaviour by directly assessing the resource's elemental composition or relying on other properties that are an indirect result of P-limitation (Cease et al. 2016). It is additionally unclear how the timespan of exposure to a given resource affects feeding behaviour. For example, in both Daphnia and nematodes, ingestion rates initially decrease when consumers are introduced to low-quality resources (Shtonda \& Avery 2006; Song et al. 2013; Lukas \& Wacker 2014) and then increase over time, ultimately exceeding the rate observed with high-quality resources. Thus, acclimation can alter the relationship between resource quality and functional responses. 
The goal of this experimental study was to test Q1) if acclimation to a novel resource alters the functional response of consumers; $\mathrm{Q} 2$ ) if $\mathrm{P}$-limitation induces compensatory feeding in the consumer, and if this response is consistent across resource densities; Q3) if $\mathrm{P}$-limitation modifies the shape (i.e., type II or III) of a consumer's functional response; and Q4) to what extent are differences in ingestion rates driven by direct or indirect effects of P-limitation. To investigate these questions, we conducted ingestion trials using the microzooplankton consumer Brachionus calyciflorus and the motile green algae Chlamydomonas reinhardtii as its resource. We used algae with both high (HP) and low phosphorus (LP) content, as well as a third resource quality created by supplementing LP-algae with inorganic $P(L P+P)$. The $L P+P$ treatment increased the $P$-content of the algae but retained the other biochemical properties associated with growth in $\mathrm{P}$-limited conditions (Boersma 2000), allowing us to disentangle the direct effects of mineral P-limitation compared to the indirect effects of biochemical deficiencies of LPalgae. This study provides insight into how changes in resource stoichiometry may impact the stability and strength of autotroph-herbivore trophic dynamics.

\section{Materials \& Methods}

\section{Rotifer and Algae Cultures}

We used the planktonic monogonont rotifer Brachionus calyciflorus as the consumer in the ingestion trials as it is an important grazer in aquatic systems, especially in ponds and lakes where fish exclude larger grazers (e.g., Daphnia) (Guijun et al. 2012). For this study, we used a single clone of $B$. calyciflorus obtained from the dormant egg bank of a Dutch pond $\left(52.02630^{\circ} ; 4.18355^{\circ}\right)$. Before the functional response trials, we maintained stock cultures of the clone at room temperature under continuous light conditions in a suspension of nutrientfree WC medium (Kilham et al. 1998) and nutrient replete algae (atomic C:P 110) at a concentration of $\sim 12 \mathrm{mgC} \mathrm{L}^{-1}$. Three times a week, we transferred a subset of the rotifers $(\sim 20 \%)$ to new containers with fresh algae suspension.

We used the green motile algae Chlamydomonas reinhardtii as the resource in our ingestion trials. In the trials, we measured feeding rates with three different food quality treatments: (i) algae cultured in high phosphorus conditions (HP, molar C:P $=109 \pm 20 \mathrm{SE}$ ), (ii) algae cultured in low phosphorus conditions (LP, molar C:P = $346 \pm 26 S E$ ) and (iii) algae cultured in LP conditions but spiked with inorganic phosphorus prior to feeding ( $\mathrm{P}+\mathrm{P}$, molar $\mathrm{C}: \mathrm{P}=88 \pm 9 \mathrm{SE})$. The $\mathrm{LP}+\mathrm{P}$ treatment combines the morphological and biochemical properties of LP-algae with the $P$ content of HP. Indeed, the HP and LP+P did not differ in C:P ratio $(p=0.32)$. As in (Zhou et al. 2018) we compared these treatments to disentangle the direct (LP vs. $L P+P)$ and indirect effects ( $L P+P$ vs. HP) of $P$ limitation. We cultured both HP- and 
LP-algae in $1.2 \mathrm{~L}$ continuous chemostats with a dilution rate of 0.25 day $^{-1}$ at $23 \pm 1^{\circ} \mathrm{C}$ in constant light. To achieve high and low phosphorus culturing conditions, we provided algae with modified WC medium with $65 \mu \mathrm{mol} \mathrm{P} \mathrm{L} \mathrm{L}^{-1}, 1000 \mu \mathrm{mol} \mathrm{N} \mathrm{L}{ }^{-1}$, and $20 \mu \mathrm{mol} \mathrm{P} \mathrm{L} \mathrm{L}^{-1}, 2000 \mu \mathrm{mol} \mathrm{N} \mathrm{L}^{-1}$ respectively. Before the initiation of the experiment, chemostats were in steady state for over six months.

\section{Experimental Design Overview}

First, we performed ingestion trials to produce functional response curves for five treatments: I) LP resource - no acclimation ( $n=52$ trials), II) LP resource -72 hours acclimation ( $n=48$ trials), III) LP+P resource - no acclimation ( $n=48$ trials), IV) LP+P resource -72 hours acclimation ( $n=40$ trials), and $V$ ) HP resource ( $n=48$ trials). We used these data to estimate the three parameters $(b, h$, and $q)$ and the associated 95\% confidence intervals of each treatment's generalized functional response model. Finally, we compared model parameters to determine the effect of acclimation, direct $\mathrm{P}$-limitation, and indirect $\mathrm{P}$-limitation.

\section{Ingestion Trials}

To generate the functional response curves of each consumer treatment, we ran 40-52 ingestion trials. We intentionally overrepresented low resource densities (<2 $\left.\mathrm{mgC} \mathrm{L}^{-1}\right)$ and included trials at very low densities ( $\left.<0.5 \mathrm{mgC} \mathrm{L}^{-1}\right)$ to accurately distinguish between Type II and III functional response curves (Sarnelle \& Wilson 2008; Uszko et al. 2020). We conducted the trials over a period of six weeks, each day we ran three to five trials from at least two experimental treatments.

Seventy-two hours prior to the ingestion trial, rotifers were transferred from stock cultures to acclimation vessels to ensure standardized conditions. As the generation time of $B$. calyciflorus is less than one day (Zhou et al. 2018), we used individuals in the trials that had been exposed to the resource treatment for at least one generation. We transferred rotifers used in the LP and LP+P acclimation treatments to vessels containing the respective algae suspension. We transferred rotifers used for the no acclimation and HP treatment to vessels containing HP algae suspension. All vessels initially contained $100 \mathrm{~mL}$ of algae suspension $\left(12 \mathrm{mgC} \mathrm{L}^{-1}\right)$ and were seeded with 1000 rotifers. We entirely replaced the algal suspension every 24 hours with the volume adjusted daily to maintain a density of 10 rotifers $\mathrm{mL}^{-1}$. Vessels were maintained in a dark incubator at $23 \pm 1^{\circ} \mathrm{C}$ on a slowly moving shaking plate (25rpm) to prevent algae from settling.

On the morning of each ingestion trial, we harvested algae from the LP and HP chemostats. After harvesting, we centrifuged the algae at $2500 \mathrm{rpm}$ for $10 \mathrm{~min}$ and resuspended the pellet in nutrient-free WC medium to ensure no $P$ was present in 
the media. To create the LP+P treatment, we added inorganic phosphate $\left(\mathrm{K}_{2} \mathrm{HPO}_{4}\right.$, $0.05 \mathrm{~mol} \mathrm{~L}^{-1}$ ) to the LP-algae suspension. We used a coulter counter (Multisizer 3TM Coulter Counter, Beckman Coulter) to determine the algal biovolume concentration $\left(\mu \mathrm{m}^{3} \mathrm{~L}^{-1}\right)$ of the resuspended algae. We used algal biovolume concentration to estimate $\mathrm{C}$ content using a previously established biovolume - $\mathrm{C}$ calibration curve. We kept all treatments in the dark for 90 min between the resuspension and ingestion trials to provided time for inorganic $P$ uptake in the $\mathrm{LP}+\mathrm{P}$ treatment. We preserved a $20 \mathrm{~mL}$ sample of each algae suspension on a GF/F filter for later elemental analysis.

Shortly before the ingestion trials, we isolated rotifers from the specified acclimation vessel and resuspended them in nutrient-free WC media. Using a stereomicroscope, we pipetted 50 adult rotifers in a volume of $50 \mu \mathrm{L}$ directly into a vessel containing $5.45 \mathrm{~mL}$ of experimental algal suspension. We recorded the "start-time" for the trial. We then took a $0.5 \mathrm{~mL}$ sample to determine the starting algal biovolume concentration $\left(\mu \mathrm{m}^{3} \mathrm{~L}^{-1}\right)$ using a coulter counter. We chose a density of 10 rotifers $\mathrm{mL}^{-1}$ because preliminary trials revealed this number of individuals was large enough to observe grazing effects at all experimental levels while allowing the consumer density to remain below $B$. calyciflorus' threshold of intraspecific interference (Fussmann et al. 2005). The experimental vessel was placed into a dark incubation chamber at $23 \pm 1^{\circ} \mathrm{C}$ on a slowly moving shaking plate (25rpm).

The incubations lasted for exactly four hours. This period was sufficient to observe a measurable decrease in algal biovolume across all concentrations but did not result in a greater than $55 \%$ decrease. At the end of the ingestion trial, we passed the whole sample over $33 \mu \mathrm{m}$ mesh to remove the rotifers. We determined the final algal biovolume concentration $\left(\mu \mathrm{m}^{3} \mathrm{~L}^{-1}\right)$ for each trial using the coulter counter. Initial and final algal biovolume concentrations were later converted to $C$ concentration using the elemental content of algae suspension collected on the same day. For each ingestion trial, we calculated the $C$ consumed $\left(C_{\text {Initial }}-C_{\text {Final }}\right)$.

\section{Elemental Analysis}

$\mathrm{C}$ and $\mathrm{N}$ content of algae were determined using a FLASH 2000 organic element analyzer (Interscience B.V., Breda, Netherlands). For $\mathrm{P}$, the algal samples were first incinerated at $550^{\circ} \mathrm{C}$ for $45 \mathrm{~min}$ and autoclaved in $2.5 \%$ potassium persulfate $\left(\mathrm{K}_{2} \mathrm{~S}_{2} \mathrm{O}_{8}\right)$ solutions at $121^{\circ} \mathrm{C}$ for $30 \mathrm{~min}$. These samples were measured using a QuAAtro segmented flow autoanalyzer (Beunde Ronde, Abcoude, the Netherlands). 


\section{Data Analysis}

For each experimental treatment, we used $C$ consumption to estimate the parameters ( $b, h$, and $q)$ of the generalized functional response model (Equation 1 ) (Real 1977). As we did not replace algae following consumption, resource depletion occurred over time (Rogers 1972). We accounted for the change in resource density through the numerical integration of the ordinary differential equation (ODE, Equation 2):

$$
\frac{d N}{d t}=-F(N) P
$$

where $P$ is the consumer density. Applying the method developed by (Rosenbaum \& Rall 2018), we fit the ODE model (Equation 2) to the observed ingestion response using an iterative maximum likelihood method, see section 2.3 in (Daugaard et al. 2019) for an in-depth description. We used a log-normal distribution in the likelihood function.

To determine confidence intervals for the functional response parameters, we used resampling methods as suggested in (Rosenbaum \& Rall 2018). For each experimental treatment, we randomly sampled with replacement the original data set 999 times and fit a functional response curve to each dataset. We used the parameter estimates from these fits to generate bias-corrected and accelerated (Bca) confidence intervals to account for bias, skew, and bounded parameters (Efron 1987; Pritchard et al. 2017). We considered the experimental treatments' parameters estimates to be significantly different if the confidence intervals did not overlap.

To answer our previously outline experimental questions, we performed the following comparisons: (Q1) to determine the effect of acclimation on consumer functional response, we compared all parameters of the LP to LP+acclimation treatment, as well as the LP+P to LP+P+acclimation treatment; (Q2/O3) To determine the effect of resource $P$-limitation we compared the parameters of the $L P$ and HP treatments, $b$ and $h$ for compensatory feeding and $q$ to determine if $P$ limitation modified the type of functional response; (O4) To determine if the response to $\mathrm{P}$-limitation were the effect of direct elemental limitation or indirect limitation we compared the model parameters of the LP and LP+P treatments.

Finally, we tested the effect of $\mathrm{P}$-limitation on $\mathrm{P}$ consumption rates. Specifically, we were interested if compensatory feeding resulted in equal $P$-consumption with LP and HP resources (Lincoln et al. 1986). Previously calculated $C$ consumption was converted to $\mathrm{P}$ consumption using the data collected on each algae suspension's elemental content. With $\mathrm{C}$ density as the predictor and $\mathrm{P}$ consumption as the response, we fitted a generalized functional response model (Real 1977). We generated $\mathrm{Bca}$ Cls of the fit (as described above) for the HP and LP resource 
treatment. We used the $\mathrm{P}$ consumed per unit $\mathrm{C}$ curves to calculate the difference in $P$ consumption between rotifers in the HP and LP treatments. If the confidence interval of this difference did not overlap with zero $\mathrm{P}$ consumption between rotifers in the two treatments was considered to be significantly different (positive: HP>LP, negative: $L P>H P$ ).

We performed all statistical analyses in the R software environment 4.0.2 (R core team 2020). For the numerical solution of the ordinary differential equations, we used the package odeintr (Keitt, 2017), and for the maximum likelihood estimation, we used the function mle2 from the package bbmle (Bolker \& Team, 2017). We used the package boot (Canty \& Ripley 2020) for both non-parametric bootstrapping and the calculation of the Bca confidence intervals.

\section{Results}

Effect of acclimation on $\mathrm{C}$ ingestion rates in $\mathrm{LP}$ and $\mathrm{LP}+\mathrm{P}$ treatments

Prior acclimation did not affect the functional response of animals exposed to LP or $L P+P$ food. Indeed, the naive and acclimated consumers' confidence intervals overlapped for each of the three functional response parameters $b, h$, and $q$ (Table S1, Figure S1). As we observed no significant effect of acclimation treatment, we combined the data from trials with naive and acclimated animals to generate a single functional response curve for each of the 'LP' and 'LP+P' treatments.

\section{Effect of resource P-limitation on $\mathrm{C}$ ingestion rates}

Consumers provided HP-algae had a significantly longer handling time $h$ (i.e., lower maximum ingestion rate) than consumers feeding on LP (Figure 1c, Table 1). However, the space clearance rate $b$ (i.e., ingestion rate at low resource densities) did not differ between HP and LP treatment (Figure 1b, Table 1). Additionally, the attack exponent $q$ did not differ between the HP and LP treatments and was significantly greater than 0 (Figure $1 \mathrm{~d}$, Table 1 ), indicating a type III functional response.

The handling time of consumers provided LP+P-algae was significantly lower than consumers with HP algae, however, it was not significantly different from consumers with LP algae (Figure 1c, Table 1). The space clearance constant $b$ and the attack exponent $q$ of consumers with LP+P resource did not differ from the other two food treatments (Figure 1b,d, Table 1). 


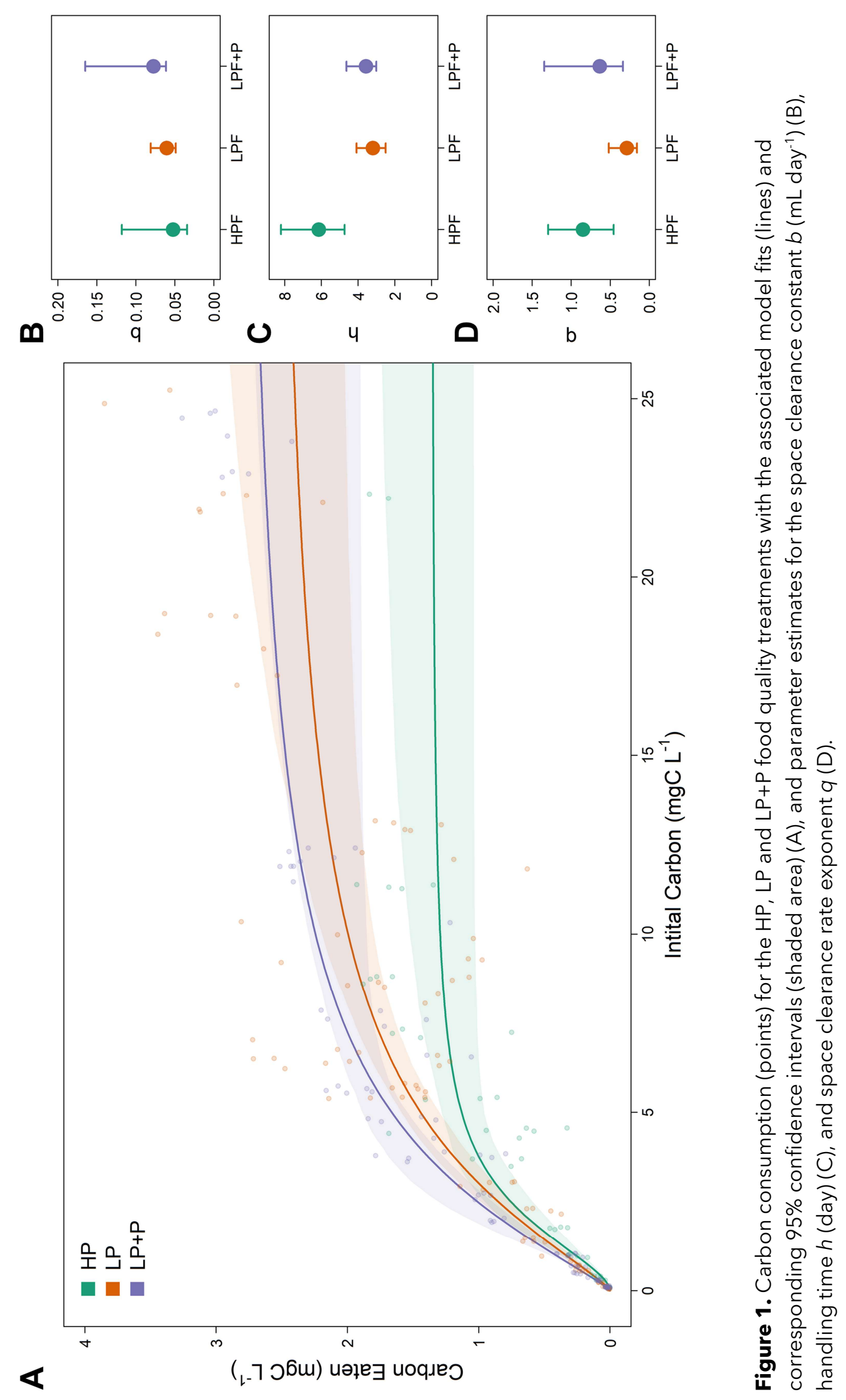

Direct and indirect effects of phosphorus limitation on the functional response of the microzooplankton consumer Brachionus calyciflorus | 119 
Table 1. Model parameter estimates, and associated bias corrected and accelerated confidence intervals for the space clearance constant $b$, handling time $h$ and space clearance rate exponent $q$. HP - high $P$ food ( $C: P=109 \pm 20 S E), L P$ - low $P$ food ( $C: P=346 \pm 26 S E$ ) and $L P+P$ - low $P$ food with $P$ supplement ( $C: P=88 \pm 9 S E)$.

\begin{tabular}{|c|c|c|c|c|c|c|c|c|c|c|}
\hline \multirow[b]{2}{*}{ Food } & \multirow[b]{2}{*}{ obs } & \multicolumn{3}{|c|}{ b } & \multicolumn{3}{|c|}{$\mathbf{h}$} & \multicolumn{3}{|c|}{$q$} \\
\hline & & Est. & Lower $\mathrm{Cl}$ & Upper Cl & Est. & Lower $\mathrm{Cl}$ & Upper Cl & Est. & Lower $\mathrm{Cl}$ & Upper Cl \\
\hline $\mathrm{HP}$ & 48 & 0.052 & 0.034 & 0.118 & 6.136 & 4.732 & 8.202 & 0.848 & 0.455 & 1.295 \\
\hline LP & 100 & 0.060 & 0.049 & 0.081 & 3.193 & 2.504 & 4.087 & 0.287 & 0.158 & 0.518 \\
\hline$L P+P$ & 89 & 0.077 & 0.061 & 0.165 & 3.562 & 3.009 & 4.636 & 0.633 & 0.337 & 1.345 \\
\hline
\end{tabular}

\section{Effect of resource P-limitation on $\mathrm{P}$ consumption}

When the initial resource density was greater than $0.16 \mathrm{mgC} \mathrm{L}^{-1}$, rotifers with HPalgae consumed significantly more $P$ than rotifers with LP-algae. This difference in $P$ consumption was greatest at $3.3 \mathrm{mgC} \mathrm{L}^{-1}$, after which it decreased logarithmically until resource intake saturated in both food qualities. There were no differences in $P$ consumption at very low initial resource densities, or our methods could not detect them.

\section{Discussion}

Our study demonstrates that herbivore consumers exhibit compensatory feeding at high but not low food densities in response to resource P-limitation. This change in ingestion rate is not directly mediated by the resource's $P$ content, rather indirectly by other properties associated with algal P-limitation. Rotifers consuming LP algae had lower handling times $(h)$ and thus higher maximum ingestion rates than those consuming HP algae, consistent with compensatory feeding. However, the space clearance rate coefficient $(b)$ of consumers did not differ between rotifers with HP- and LP-algae, indicating that compensatory feeding does not occur at low food densities. Furthermore, we observed no differences in the functional response of consumers provided with LP and LP+P algae, despite the former being $P$-limited and the latter being $P$-replete. Thus, the rotifers' food ingestion rate appears to respond to properties of the resource that P-limitation indirectly determines (e.g., biochemical composition, morphology) rather than to the mineral $P$-content of the algae itself. We observed no difference in ingestion rates between individuals naive or acclimated to LP/LP+P algae, suggesting that food ingestion rates can be rapidly modified in response to changes in food quality. Finally, all resource treatments were consistent with a Type III functional response suggesting that changes in food stoichiometry will not affect consumerresource interactions' stability through changes in functional response type. 


\section{Compensatory feeding with LP resources}

Studies investigating the effect of P-limitation have provided support for compensatory feeding at high resource densities for a variety of aquatic grazers [e.g., Daphnia, (Plath \& Boersma 2001); Copepods, (Burian et al. 2018); Rotifers, (Zhou \& Declerck 2019); Snails, (lannino et al. 2019)], whereas empirical data documenting such a response at low food availabilities is largely lacking. In line with other studies, at saturating food concentrations, we observed that the maximum ingestion rate of consumers with LP-algae was significantly higher than those with HP-algae, as indicated by a lower handling time $h$. However, we observed no difference in the space clearance rate coefficient $b$, indicating that there were no differences in $\mathrm{C}$ consumption at low food densities.

There may be several complementary explanations for the observed lack of compensatory feeding at low food densities. First, at very low C-concentrations, organisms allocate all ingested $\mathrm{C}$ (energy) to basal metabolic and growth requirements, thus none is available to allocate towards activities, such as greater movement, that would increase the space clearance rate (Suzuki-Ohno et al. 2012). Additionally, as the energy expenditure to find resources is inversely proportional to resource abundance (i.e., when food is scarce, searching requires a high energy investment), when $\mathrm{C}$ concentrations are low, organisms may down regulate searching to conserve energy (Abrams 1982). Although this strategy is effective for only a limited period, it can be beneficial for organisms living in resource environments that are temporally or spatially heterogeneous. Finally, when $C$ consumption is low, organismal growth is reduced, decreasing demand for $\mathrm{P}$ (Sterner and Robinson 1994). Thus, at low $C$ concentrations, compensatory feeding of resources with a high $\mathrm{C}: \mathrm{P}$ is not yet necessary as $\mathrm{P}$ is consumed in excess of the low organismal requirements. In our study, significantly higher $C$ consumption in response to $\mathrm{P}$-limitation was observed at resource densities greater than approximately five $\mathrm{mgC} \mathrm{L}^{-1}$, suggesting that this is the point at which P-limitation replaces $\mathrm{C}$-limitation. The results of this study expand on previous work regarding the effect of food quantity and quality on herbivores ingestion rates (Fink \& Von Elert 2006) and provide insight into how this behaviour changes across a gradient of food availability concentrations.

Despite compensatory feeding at high food densities, rotifers with low $P$ resources still had a significantly lower intake of $P$ than rotifers with high $P$ resources. The inability to increase consumption of a low-quality resource to compensate for a limiting nutrient has been seen in other zooplankton (Burian et al. 2018) and suggests that although modifications of ingestion rates can partially mitigate the fitness effects of nutrient limitation, physiological constraints may limit its effectiveness. It is important to note that $\mathrm{P}$ intake (i.e., $\mathrm{P}$ content of resource 
consumed) does not necessarily reflect the P-assimilated by the consumer.

Consumers increase ingestion rates by decreasing their gut retention time (Jumars 2000). This reduced gut retention time comes at the cost of decreased assimilation efficiency (DeMott et al. 1998); however, as more particles are consumed, the net intake of $P$ is still higher than at lower ingestion rates (Jumars 2000; Burian et al. 2020). Due to lower assimilation efficiencies, the actual difference in P-

consumption between consumers with high- and low-P resources will be greater than expected based on the P-content of the resource alone. Physical limitations on ingestion and assimilation likely explain the consistent significant differences in fitness between individuals consuming high- and low-P resources, despite the observation of compensatory feeding (Fink \& Von Elert 2006; Flores et al. 2014; Zhou \& Declerck 2019).

\section{Food $\mathrm{P}$ content is not the cue for compensatory feeding}

We observed that at saturating food quantities, food ingestion rates of rotifers provided LP+P-algae (i.e., LP-algae spiked with inorganic $P$ ) were significantly higher than those of rotifers with HP-algae, despite similar C:P ratios of the food treatments. In contrast, the functional responses of rotifers with LP+P-and LPalgae, two treatments with the same growth history of $\mathrm{P}$-limitation but with different $C: P$ ratios, were indistinguishable. We propose two non-exclusive explanations for this finding. The first is that rotifers use algal properties associated with $\mathrm{P}$-limitation and not elemental content to assess resource nutritional quality and regulate feeding behaviour (Simpson \& Raubenheimer 2012). History of growth under P-limiting conditions may alter physical properties of algae cells such as taste, palatability, or macronutrient content, which serve as reliable cues to its elemental content. In this experiment, P-supplementation breaks this association, and rotifers can no longer accurately infer the amount of $P$ being ingested.

Alternatively, rotifer growth is not only limited by $P$ but also by other substances. For example, compared to P-replete algae, P-limited algae have been shown to contain fewer highly unsaturated fatty acids (HUFAs) such as EPA, ARA, and DHA (Müller-Navarra 1995; Boersma 2000), which are essential for growth and reproduction in animals (Stanley-Samuelson 1994). P supplementation of LP-algae occurs immediately before feeding and, as such, does not affect the concentration of other nutritious molecules in the algae (Boersma 2000). Therefore, compensatory feeding would still be necessary to compensate for the reduced amount of such molecules despite the abundance of $P$ in LP+P algae. The latter explanation is supported by (Zhou et al. 2018), who found that the fitness of rotifers provided LP+P algae was intermediate between those fed high and low $P$ algae. This suggests that the low nutritional value of algae with a growth history of $P$ limitation is not entirely compensated by the addition of $P$. 
Previous experiments investigating the effect of stoichiometric imbalance on ingestion rates found, in contrast with this study, that the ingestion rate of consumers with LP+P algae was lower than algae with a history of growth in Plimiting conditions [Daphnia: (DeMott et al. 1998); B. calyciflorus (Zhou \& Declerck 2019)]. Dissimilarities between this study and DeMott et al. (1998) may be attributed to species-specific differences at both the consumer (Daphnia magna vs B. calyciflorus) and resource (Synechococcus elongatus vs. C. reinhardtii) level. For example, the cues for P-limitation may differ both in how they are detected in the consumer and how they manifest in the resource. Although the same species were used in this study and (Zhou \& Declerck 2019) both the genotype and algal strain differed between experiments.

\section{Effect of Acclimation}

Acclimation to low quality resources did not affect the functional response of $B$. calyciflorus, suggesting that rotifers are able to rapidly modify their ingestion rates in response to food quality. Consistent with our study, an investigation that compared the functional response of consumer populations cultured in contrasting conditions prior to the ingestion trials, observed that the estimates of parameters $(h, b$, and $q$ ) did not significantly differ between food treatments (Uszko et al. 2015). Despite the perception that aquatic environments are homogenous, zooplankton consumers may face considerable changes in food quality both within a generation and seasonally (Hessen et al. 2005; Sommer et al. 2012). In order to persist in heterogeneous environments organisms require the capacity to rapidly adjust their physiological responses. Thus, the similarities in functional response between the naive and acclimated individuals in the LP and LP+P treatments are not surprising.

\section{Type III functional response}

In all three food quality treatments we observed functional responses that were consistent with a Holling's type III. Our results add to a growing body of work indicating that type III responses are commonplace in rotifers (Fussmann et al. 2005; Seifert et al. 2014; Colina et al. 2016), and consumers in general (Sarnelle \& Wilson 2008; Kalinkat et al. 2013; Sarnelle et al. 2015). Discriminating between type II and III responses is important for understanding the stability of consumer resource interactions. However, doing so requires an experimental design that deliberately ensures that tested food densities vary by orders of magnitude (Uszko et al. 2020), and a third of which should be below the organism's R* (Uszko et al. 2015). Previous observations of type II responses in B. calyciflorus (Rothhaupt 1990; Verschoor et al. 2007) may be the result of a lack of such low density data (Sarnelle \& Wilson 2008). Changes in environmental factors such as temperature 
may shift a consumer's foraging response from a type III to type II (Taylor \& Collie 2003; Daugaard et al. 2019). However, in both high and low P conditions $B$. calyciflorus display a type III response, suggesting that modifications in the shape of the functional response due to resource stoichiometry are unlikely to destabilize consumer-resource interactions.

\section{Conclusion}

Our results provide insight into the effect of stoichiometry on the ingestion rate of the rotifer $B$. calyciflorus. We found that changes in resource stoichiometry do not shift consumers away from density-dependent search rates (i.e., type III response), but do alter maximum ingestion rates and result in compensatory feeding with low $P$ food at saturating resource densities. These results suggest that consumerresource interactions may remain stable in the face of changes in elemental availability. However, in systems with high $C$ availability, increase in autotroph $C: P$ may increase top-down control of $B$. calyciflorus because of compensatory feeding. Furthermore, the observation that rotifers display compensatory feeding with LP+P algae, despite its high $\mathrm{P}$-content, highlights gaps in knowledge regarding the mechanisms regulating ingestion. Our study demonstrates that for herbivore consumers there is a significant interaction between resource quality and quantity on food ingestion rates, however, such dynamics are rarely taken into consideration in predictive ecological modelling. The incorporation of stoichiometrically explicit functional responses, especially in highly productive systems, will aid in our understanding of trophic interactions, and the impacts of anthropogenic changes in nutrient cycling.

\section{Acknowledgements}

This work was supported by grant $n^{\circ} 823.01 .011$ of the Earth and Life Sciences Division (ALW) of the Netherlands Organization for Scientific Research (NWO). We wish to thank Zarah Sharif for laboratory assistance throughout the study, Nico Helmsing for carrying out nutrient analyses. We also thank Cam Hudson, and Celia Symons for constructive comments on the manuscript. 


\section{Supplementary Materials}

\section{Supplementary Tables}

Table S1. Model parameter estimates and associated bias corrected and accelerated confidence intervals for the space clearance constant $b\left(\mathrm{~mL} \mathrm{day}^{-1}\right)$, handling time $h($ day), and space clearance rate exponent $q$ of naive (exposure prior to ingestion trial $=0 \mathrm{~h}$ ) or acclimated (exposure prior to ingestion trial $=72 \mathrm{~h}$ ) rotifer consumers. $L P$ - low $P$ food $(C: P=346 \pm 26 S E)$ and $L P+P-\operatorname{low} P$ food with $P$ supplement ( $C: P=88 \pm 9 S E)$.

\begin{tabular}{|c|c|c|c|c|c|c|c|c|c|c|c|}
\hline \multirow[t]{2}{*}{ Food } & \multirow{2}{*}{$\begin{array}{c}\text { Exposure } \\
\text { (h) }\end{array}$} & \multirow[t]{2}{*}{ obs } & \multicolumn{3}{|c|}{ b } & \multicolumn{3}{|c|}{$h$} & \multicolumn{3}{|c|}{$q$} \\
\hline & & & Est. & $\begin{array}{c}\text { Lower } \\
\mathrm{Cl}\end{array}$ & $\begin{array}{c}\text { Upper } \\
\mathrm{Cl}\end{array}$ & Est. & $\begin{array}{c}\text { Lower } \\
\mathrm{Cl}\end{array}$ & $\begin{array}{c}\text { Upper } \\
\mathrm{Cl}\end{array}$ & Est. & $\begin{array}{c}\text { Lower } \\
\mathrm{Cl}\end{array}$ & $\begin{array}{c}\text { Upper } \\
\mathrm{Cl}\end{array}$ \\
\hline LP & 0 & 52 & 0.063 & 0.049 & 0.082 & 3.309 & 2.607 & 4.195 & 0.325 & 0.15 & 0.524 \\
\hline LP & 72 & 48 & 0.055 & 0.037 & 0.145 & 2.963 & 1.766 & 5.017 & 0.232 & 0.032 & 1.924 \\
\hline$L P+P$ & 0 & 48 & 0.091 & 0.072 & 0.377 & 3.337 & 2.743 & 4.668 & 0.465 & 0.222 & 2.224 \\
\hline$L P+P$ & 72 & 40 & 0.077 & 0.048 & 2.871 & 4.065 & 2.999 & 6.505 & 0.941 & 0.382 & 3.716 \\
\hline
\end{tabular}




\section{Supplementary Figures}
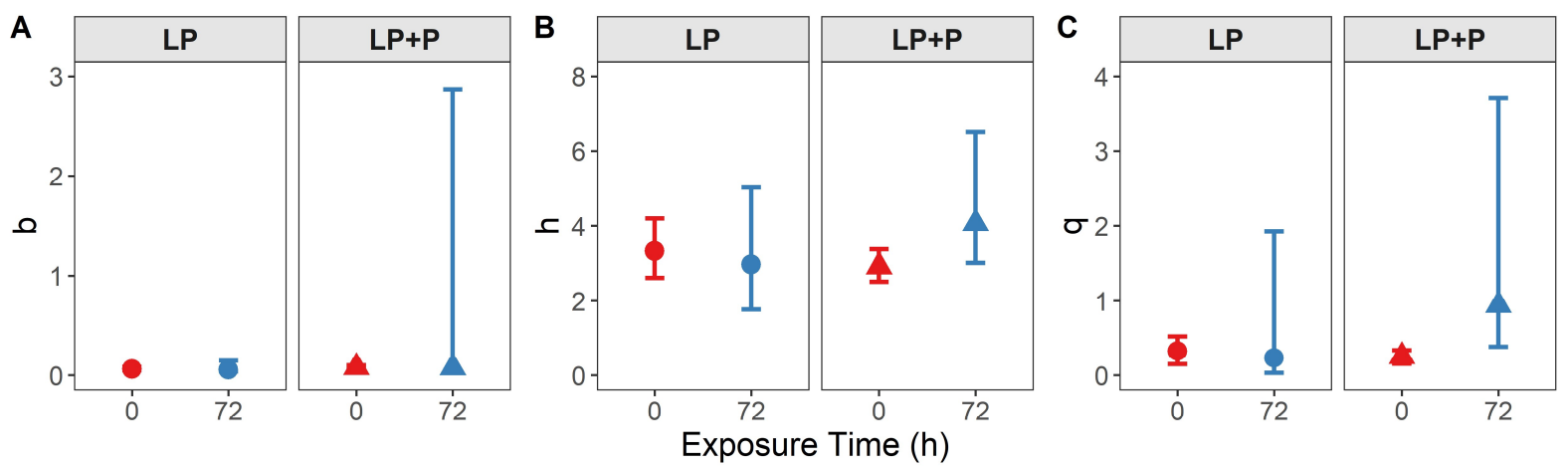

Figure S1. Parameter estimates (A) clearance constant $b\left(\mathrm{~mL}\right.$ day $\left.{ }^{-1}\right),(B)$ handling time $h$ (day), and (C) space clearance rate exponent $q$ for rotifer consumers either naive (exposure time prior to ingestion trial $=0 \mathrm{~h}$ ) or acclimated ( Exposure time $=72 \mathrm{~h}$ ) to non-HP resources. LP - low $P$ food (C:P=346 $\pm 26 S E)$ and $L P+P-$ low $P$ food with $P$ supplement ( $C: P=88 \pm 9 S E)$.

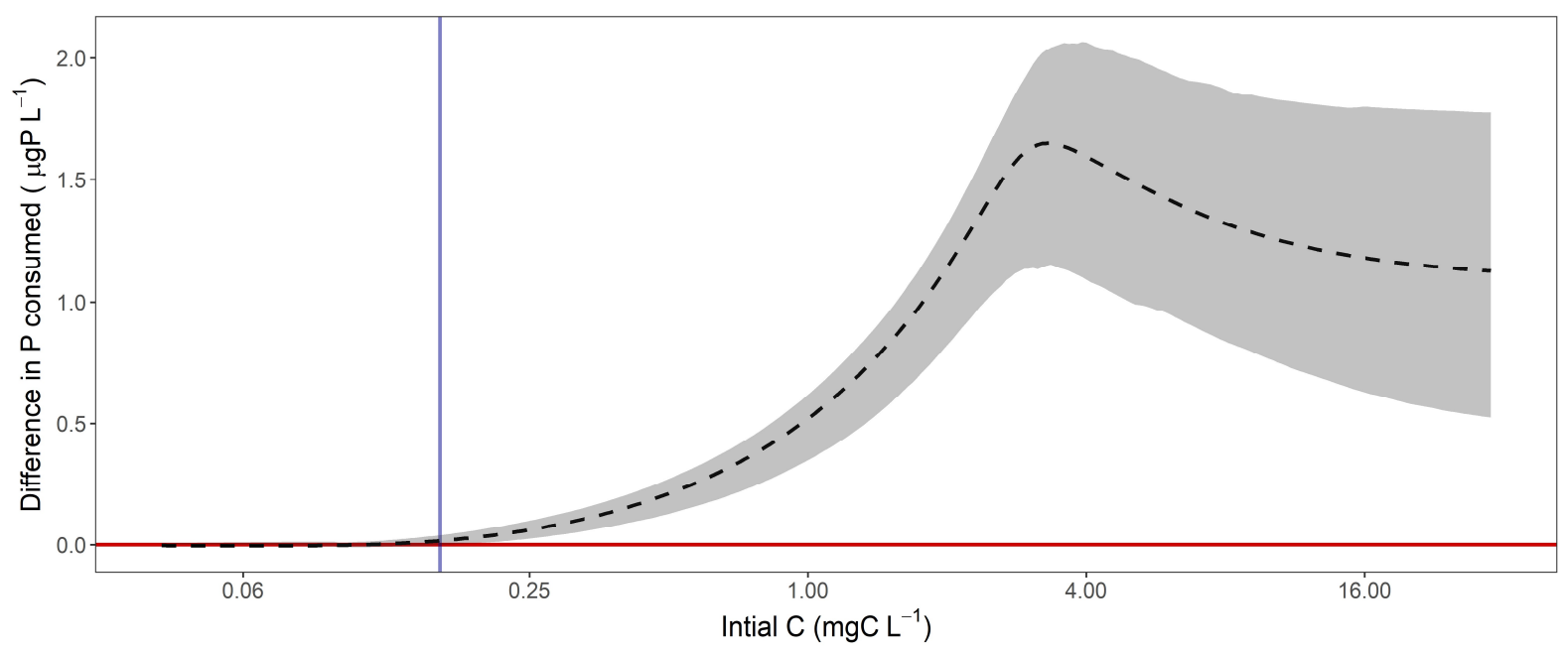

Figure S2. Difference in $\mathrm{P}$ consumption and associated $95 \% \mathrm{Cl}$ (shaded area) between rotifers consuming HP- and LP-algae on the linear-log2 scale. The red line represents no difference in $\mathrm{P}$ consumption, the blue line represents the point at which $\mathrm{P}$ consumption is significantly greater in the HP than the LP-algae. 




\section{Chapter 6}

Synthesis 

Organisms need to obtain elements from the environment in specific ratios to grow and reproduce, yet the availability of these elements often does not match their requirements. While such stoichiometric mismatches can pose a challenge for consumer fitness, elemental limitation may be mitigated by adaptive phenotypic changes. The ecological stoichiometric framework has primarily been used to advance our understanding of the physiological (Darchambeau 2005) and community (Moody \& Wilkinson 2019) impacts of such mismatches. Despite early calls for the use of ecological stoichiometry to understand evolutionary patterns (Kay et al. 2005; Elser 2006), and later proposals on its utility in decomposing ecoevolutionary feedbacks (Matthews et al. 2011; Leal et al. 2017b), few studies conducted within this framework have incorporated evolution.

This thesis aims to further incorporate an evolutionary perspective into the ecological stoichiometric framework to understand how stoichiometric mismatches shape the phenotypes of primary consumers on ecologically relevant timescales. I explored this question by combining literature and lab-based methods with a focus on experimental evolution. First, using a meta-analysis approach, I documented substantial intraspecific variation in stoichiometric traits, establishing the potential for evolutionary change (Chapter 2). I then tested the ability of the ecological stoichiometric framework to predict microevolutionary changes in body stoichiometry in response to selection for fast growth (i.e., the growth rate hypothesis) in high and low resource environments. I found that the growth rate hypothesis can predict evolutionary trajectories, but that its success is dependent on the environmental context under which selection takes place, as well as the genetic background of the genotypes under consideration (Chapter $\mathbf{3}$ ). Although standing genetic variation, which was present in Chapter 3, is typically considered necessary for rapid adaptation, some genetically depauperate populations are successful in the face of environmental change. I therefore wanted to investigate whether a heritable adaptive response could be observed in populations that initially lack standing genetic diversity. I found, contrary to present dogma, that such a response is possible, but is also highly genotype dependent (Chapter 4). Finally, as resource quality is both spatially and temporally heterogeneous, consumers may experience various degrees of stoichiometric mismatch within their lifespan. Thus, I investigated plastic phenotypic changes in consumer ingestion rates in response to stoichiometric mismatch. I observed that consumers provided low $\mathrm{P}$ resources displayed compensatory feeding in high, but not low, resource densities, and that changes in ingestion rate are not directly mediated by the resource's $P$ content (Chapter 5). In this synthesis, I connect the findings from the preceding chapters into four main takeaway messages. This is followed by a short outlook on prospective research directions in ecological stoichiometry and concludes by summarizing the findings of this thesis. 


\section{Takeaway Messages}

There is substantial intraspecific variation in stoichiometric traits

Ecologists often focus on differences between co-occurring species, and by doing so they tend to neglect the existence of intraspecific variation (Violle et al. 2012). However, investigations of the extent of trait variation within species and populations demonstrates that such variation is frequently considerable, and can have significant impacts on ecosystem processes (Hughes et al. 2008; Des Roches et al. 2018). Despite this, a reductionist approach to species traits persists in the ecological stoichiometric framework, especially for heterotrophs such as primary consumers (Sterner 1990; Branco et al. 2010; Suzuki-Ohno et al. 2012; Yamamichi et al. 2015). As such, population traits are often considered fixed. The metaanalysis presented in Chapter $\mathbf{2}$ indicates that variation in stoichiometric traits (composition, acquisition, assimilation, allocation, and excretion) is substantial, and for some traits is similar in magnitude to that of life history traits which are commonly considered in microevolutionary studies. The ubiquity of genetic variation in stoichiometric traits was also observed in the elemental body composition of experimental genotypes (Chapter $\mathbf{3}$ ), as well as in the capacity to produce de novo heritable adaptive phenotypes (Chapter 4). The consistent observation of intraspecific variation within my experiments and across the surveyed literature indicates that the current approach to treat traits as "mean values per species" results in the underestimation of stoichiometric niches (González et al. 2017). Failing to document intraspecific variation hinders predictive capacities of ecological models (Violle et al. 2012) and impairs investigations of eco-evolutionary dynamics where standing genetic variation is widely considered the fuel for rapid microevolution (Barrett \& Schluter 2008).

The extent of intraspecific variation documented in Chapter 2 suggests significant potential for stoichiometric traits to rapidly evolve and impact ecosystem processes. For example, excretion, one of the primary mechanisms by which animals impact nutrient cycling within ecosystems (Vanni 2002), shows considerable intraspecific variation at both the genotype and population level (Chapter 2). A microevolutionary shift within a population that changes the relative abundance of excreted elements can significantly impact ecosystem function (Jeyasingh et al. 2014). Chowdhury and Jeyasingh (2016) provided evidence for such an effect by demonstrating that the biomass of algae cultured with high Precycling Daphnia genotypes was greater than that of algae cultured with low Precycling genotypes. The genotypes used in Chowdhury and Jeyasingh (2016) originated from the same population but at two different points in time (high-P recycling: eutrophic conditions, low-P recycling: oligotrophic conditions), 
suggesting that microevolution within a consumer population can impact the primary productivity of a system.

There have been few investigations of the ecosystem level effects of intraspecific variation in excretion and other "homeostasis maintenance traits" (i.e., acquisition, allocation, and assimilation) that have the potential to alter elemental availability. Future work aimed at documenting the extent of intraspecific variation, and thus the potential for evolutionary change, should focus on these traits and be conducted across a variety of taxa. Further empirical investigations are also needed to quantify the effect of microevolution in traits that alter elemental availability on population, community, and ecosystem interactions and how the effect compares to community composition changes.

\section{Stoichiometric mismatches are important in shaping microevolutionary}

\section{trajectories}

Phenotypes are shaped by selection, which favours traits that elevate individual fitness (i.e., greater reproduction and survival), and these traits are constrained by both the environment and an organisms' phylogenetic background. There has been considerable research on the role of genetic constraints (Futuyma 2010) in shaping microevolution, with far less attention having been paid to constraints imposed by the environment. All traits require certain biomolecular compounds that have a set stoichiometric composition, and if the elements necessary for the compounds are not available in the amounts or relative abundances necessary, the expression of that trait is constrained (Sterner \& Elser 2002). While several studies have provided correlative evidence suggesting elemental availability is a selective force that actively shapes phenotypes (e.g., Elser et al. 2006; Swanson et al. 2016; Snell-Rood et al. 2020), there have only been a limited number of direct tests of this hypothesis so far (Declerck et al. 2015; Turner et al. 2017; Archambeault et al. 2020).

This thesis provides conclusive evidence for the role of the stoichiometric environment in shaping evolutionary trajectories. As described in Chapter $\mathbf{3}$, rotifer populations selected for fast growth were provided either high-P (HP) or low-P (LP) resources. I observed that selected populations from both conditions had population growth rates that were significantly greater than the ancestral state. However, in HP-selected populations, increases in population growth rates were associated with a significant increase in body $P$, whereas no change in relative body $\mathrm{P}$ was observed in the LP-selected populations. As described in Chapter 4 , monoclonal populations were exposed to a culturing regime that favoured phenotypic variants with fast growth and again provided with HP or LP resources. Populations with an LP-exposure history displayed a heritable adaptive response in 
the common garden, while no such response was observed in populations with an HP-exposure history. In both experiments, populations exposed to the same selection pressure (i.e., fast population growth rate) displayed alternative phenotypes that were shaped by the degree of mismatch between consumer and the resource.

Identifying the factors that influence the microevolutionary trajectories of natural populations is notoriously difficult (MacColl 2011). Controlled lab studies, such as those performed in this thesis, are powerful tools that simplify nature and allow for a mechanistic understanding of ecological and evolutionary processes (Jessup et al. 2004; Benton et al. 2007). While this thesis supports the hypothesis that elemental availability shapes evolutionary trajectories, it does so in a specific environmental context (i.e., P-limitation with all other elements in excess). Testing the influence of elemental availability on evolutionary trajectories for other elements and in a diversity of taxa, as well as investigating how stoichiometric mismatches interact with other selection pressures, would allow us to further explore the generalities of these findings. Such work can be done in controlled lab experiments by adding layers of complexity (Luijckx et al. 2017), providing greater biological realism while maintaining mechanistic understanding of the system. Identifying the influence of stoichiometric mismatch in natural environments may also be possible by employing resurrection ecology techniques. Resurrection ecologists hatch dormant propagules that have been stored in egg banks in order to assess the phenotypic characteristics of organisms from discrete time periods, generally to study how populations evolve under the forces of ecological change (Burge et al. 2018). A potential avenue to investigate the role of stoichiometric mismatch in evolutionary trajectories is to collect dormant propagules from the periods before and after a significant shift in resource stoichiometry (e.g., cultural eutrophication). If large numbers of individuals can be hatched and cultured in the lab, replicate populations representative of the genetic diversity present in the "before" period could be exposed to a hypothesized selective agent (e.g., decrease C:P resources) in a mesocosm setting. If the phenotypes of the selected populations match those of the individuals from the "after" period in the sampled population it would indicate the stoichiometric mismatch was indeed the primary selective agent shaping the evolutionary trajectory.

\section{Cryptic evolution occurs in response to phosphorus limitation}

A striking observation of this thesis is that in both the experimental evolution studies (Chapter 3 and Chapter 4), LP-selected populations outperformed HPselected populations when provided LP-resources, whereas the performance of populations with contrasting selection histories did not differ when provided with HP-resources. Two previous studies investigating adaptive responses to $\mathrm{P}$ - 
limitation by Frisch et al. (2014) and Declerck et al. (2015) observed the same pattern using different experimental approaches. This pattern is indicative of cryptic evolution, which is observed when microevolution occurs within a population, but the phenotype expressed is consistent with the expectations of a purely ecological response (Cooke 1990). Thus, the evolutionary change is difficult to observe. In Frisch et al. (2014) and Declerck et al. (2015), as well as in Chapter 3 and Chapter 4, microevolution in the LP-selected populations is masked by their lower performance in LP-resources compared to populations with HP-resources. As this pattern is consistent with the ecological expectation that P-limitation will negatively affect consumer growth rates (Elser et al. 2001; Seidendorf et al. 2010; Zhou et al. 2018).

The impact of cryptic evolution is often overlooked in eco-evolutionary dynamics, however, it can have significant effects on ecosystem patterns and processes (Kinnison et al. 2015). For example, populations adapted to low $P$ environments have higher population growth rates (Chapter $\mathbf{3}$ and Chapter $\mathbf{4}$ ) and carrying capacity (Declerck et al. 2015) than non-adapted populations when $P$ is limiting, which can significantly impact trophic dynamics. Accounting for evolutionary responses that counter negative environmental effects may help to anticipate the impacts of environmental change (Kinnison et al. 2015). For example, reduction of anthropogenic $\mathrm{P}$-inputs in response to policy changes has led to the reoligotrophication of many temperate lakes (Stockner et al. 2000; Hartwich et al. 2012; Jeppesen et al. 2002). Ecosystem forecast models that fail to incorporate the capacity of primary consumers to adapt to reductions in $\mathrm{P}$-availability may underestimate the standing biomass of these populations. The consistency of such cryptic evolution in experiments investigating adaptation to P-limitation suggests such responses may be a general, although underappreciated, evolutionary pattern in aquatic consumers. Given the implications on ecosystem functioning, the extent of cryptic evolution in response to P-limitation deserves further investigation.

To predict adaptive and physiological responses to elemental mismatches in a diversity of contexts the ecological stoichiometric framework requires further development

The rapidly accelerating pace of anthropogenic environmental change has led to an intensified interest in developing frameworks capable of predictions regarding impacts on the stability and persistence of populations (Mouquet et al. 2015). Ecological stoichiometry has aided in the understanding of diverse ecosystem processes, providing a framework to study biotic and abiotic interactions by investigating the flow of elements in natural systems (Sterner \& Elser 2002). However, its capacity to predict adaptive responses to elemental mismatch 
between trophic levels has not previously been tested. Chapter $\mathbf{3}$ of this thesis specifically addresses this question by testing the predictions of the growth rate hypothesis, a central concept in ecological stoichiometry, in low and high Pavailability environments. The anticipated positive relationship between body Pcontent and growth rate was observed in HP-selected populations, but not in LPselected or hybrid populations. In the context of physiological responses, the stoichiometric framework has been well tested and generally proven successful. Thus, it was surprising to observe that, contrary to the foundations of the ecological stoichiometric framework, modifications in ingestion rate did not appear to by cued by direct elemental P-limitation (Chapter 5). However, the finding that compensatory feeding with low-P resources was not observed at low food densities aligns well with theory (Suzuki-Ohno et al. 2012).

The results of this thesis raise the question as to why the ecological stoichiometric framework is appropriate as a predictive tool in some contexts but not others. The strength of ecological stoichiometry is its ability to reduce complex interactions into their most fundamental parts (i.e., elements), however, this approach may be too reductionistic to effectively anticipate complex organismal responses. For example, some biologically important molecules such as omega-3 and omega-6 polyunsaturated fatty acid have the same elemental composition, but have different physiological effects (Ahlgren et al. 2009). Now that a basic framework has been established, and proven successful, it is time to identify places where the incorporation of greater biological realism may increase the predictive capacity of ecological stoichiometry. Incorporating intraspecific variation into ecological models in place of species means (Violle et al. 2012), especially for traits related to the maintenance of body stoichiometry (Chapter 2), will strengthen the predictive capacity of the framework. Furthermore, determining how stoichiometric traits are pleiotropically related to one another is of particular interest (Jeyasingh et al. 2014; El-Sabaawi et al. 2016) if we wish to determine how evolutionary trajectories may be constrained. The framework could further be expanded by determining what species interactions can be predicted by purely elemental quantities and when secondary properties, such as resource biochemical composition (Müller-Navarra 1995; Piepho et al. 2010), need to be taken into consideration. While no one framework will ever be a panacea for predicting organismal responses, ecological stoichiometry currently represents our most effective approach to understand the impacts of elemental mismatch across trophic levels, and has been successful in other contexts (Sanders \& Taylor 2018). 


\section{Future Developments in Ecological Stoichiometry and Beyond}

This thesis demonstrates the utility of the ecological stoichiometric framework for anticipating physiological and heritable adaptive responses to stoichiometric mismatches. However, further investigations are required to understand how the stoichiometric environment shapes phenotypes, and how these phenotypes subsequently affect ecosystem function. Such developments are crucial to anticipate the impacts of complex changes in elemental availability that are occurring because of anthropogenic environmental change.

The ecological stoichiometric framework has primarily been used to investigate the effects of species differences on ecological processes (Elser et al. 1988), with the effects of intraspecific variation having received only limited attention. Intraspecific variation has been demonstrated to impact responses across multiple levels of organization including population productivity, community diversity and nutrient cycling (Hughes et al. 2008). Furthermore, the effect of intraspecific variation on ecological dynamics (e.g., species abundance, changes in biomass, nutrient fluxes) are similar to interspecific effects in many systems (Des Roches et al. 2018; Raffard et al. 2019). Therefore, by neglecting individual level differences in stoichiometric traits as documented in Chapter 2, we may miss the variation that is responsible for ecological patterns. The stoichiometric framework also provides an opportunity to gain a mechanistic, rather than phenomenological, understanding of the effects of intraspecific diversity by tracing the flow of elements between trophic levels. For example, by experimentally manipulating the genotypic diversity of populations composed of consumers with varying excretion ratios (e.g., N:P release), we can gain insights on how intraspecific variation effects overall nutrient cycling within a system and how it can impact other trophic levels.

The ecological stoichiometric framework has played a prominent role in understanding how elemental availability impacts consumers. Studies have investigated phenotypic plasticity in response to $\mathrm{N}$ and $\mathrm{P}$-limitation in a variety of traits including ingestion (Sterner \& Smith 1993; Rothhaupt 1995; Burian et al. 2018), assimilation (Darchambeau 2005; Ferrão-Filho et al. 2007), respiration (Jensen et al. 2006; Jensen \& Hessen 2007) and excretion (He \& Wang 2007). An exciting avenue of research that deserves future investigation is documenting the mechanisms of heritable adaptation to elemental limitation. One study system where the potential of this line of investigation has been established is in the work of Frisch et al. (2014) who hatched Daphnia genotypes from oligotrophic and eutrophic periods from the same lake to study P-use efficiency. Subsequent work investigated gene expression and nutrient cycling differences between these genotypes (Roy Chowdhury et al. 2015; Chowdhury \& Jeyasingh 2016). 
Documenting the mechanisms of adaptation, and how they interact, would represent an important step forward in understanding the eco-evolutionary consequences of stoichiometric mismatch. For example, LP-adapted populations may increase $P$ intake by increasing ingestion rates and decreasing assimilation efficiency (Jumars 2000). The ecosystem effect of such an adaptation is unclear as increased ingestion rates may supress primary production, whereas decreased assimilation efficiency increases elemental excretion, which in turn has the potential to increase primary production. Such an example illustrates how adaptive mechanisms may interact and emphasizes how ecosystem effects are not straightforward. Thus, predicting the impacts of adaptation requires disentangling the different mechanisms to estimate the overall effect. An additional complexity in prediction is that there are potentially many pathways organisms could utilize to mitigate the effect of limitation, each with their own ecosystem effects. By documenting mechanisms of adaptation in a diversity of organisms and environments, there is potential to determine if consumers display a general adaptive response to elemental limitation.

One far off, but potential, implication of this work is managing P use in global food production. The use of $\mathrm{P}$ in fertilizer is critical for contemporary agricultural methods, however $\mathrm{P}$ is a finite resource and is projected to be depleted in the next 50-100 years (Cordell et al. 2009). One possible avenue to delay the time to depletion is to increase the $\mathrm{P}$ growth efficiency in agriculture. Understanding the mechanisms favoured by natural selection to P-limitation may give insight on how to optimize yields through artificial selection.

The de novo heritable adaptive phenotypic change observed in replicate populations, as described in Chapter $\mathbf{4}$, is a very exciting finding, and presents the opportunity for many fruitful lines of future investigation. Selection on standing genetic variation is considered the primary mechanism by which rapid adaptation occurs in natural populations. However, the observation of a heritable adaptive response in a population that initially lacked standing genetic variation (Chapter 4) suggests there are alternative pathways to adaptation. Documenting such a response is just the first step of many, but it may shift our perspective on the process of adaptation. It raises the question of what mechanism the de novo heritable adaptive response could be attributed to. Both environmentally-induced genetic modifications (Rey et al. 2016) and non-genetic heritable phenotypes (Bonduriansky \& Day, 2018) have been proposed as alternative pathways for rapid adaptation. In-depth molecular studies could determine proximate causes of the observed adaptive response. Another question of interest is the generality of the capacity to produce a de novo heritable response. One of the two genotypes investigated in this thesis demonstrate this capacity (Chapter 4), providing proof 
of concept that such a response is possible, but provides no indication of how commonplace it may be. Future studies should investigate this question using more genotypes as well as a variety of taxa. By studying organisms from a diversity of environments and phylogenetic backgrounds, we could gain insight on the conditions that select for the production of de novo heritable responses at both micro and macroevolutionary scales. Furthermore, by documenting the prevalence of this capacity within populations and species, we can better understand how likely this mechanism impacts ecological processes and under what circumstances. An additional line of inquiry would be to document the magnitude of traits shifts generated by de novo heritable adaptive responses. For genetically depauperate populations, mechanisms alternative to selection on standing genetic variation may enable persistence in the face of environmental change, but the potential "buffering" capacity of this response is unknown.

\section{Conclusion}

This thesis provides comprehensive evidence that primary consumers can mitigate the effects of stoichiometric mismatch through both heritable and non-heritable mechanisms. It demonstrates that: i) substantial intraspecific variation exists in stoichiometric traits; ii) stoichiometric mismatches shape microevolutionary trajectories; iii) cryptic evolution occurs in response to phosphorus limitation; and iv) the ecological stoichiometric framework can be used to predict phenotypic responses but requires further development to be a more general framework. This work highlights the need to incorporate biological realism to understand how organisms will adapt to changes in elemental availability. 

Chapter 7

References 

Abrams, P.A. (1982). Functional Responses of Optimal Foragers. Am. Nat., 120, 382-390.

Acharya, K., Kyle, M. \& Elser, J.J. (2004). Biological stoichiometry of Daphnia growth: An ecophysiological test of the growth rate hypothesis. Limnol. Oceanogr., 49, 656-665.

Ahlgren, G., Vrede, T. \& Goedkoop, W. (2009). Fatty Acid Ratios in Freshwater Fish, Zooplankton and Zoobenthos - Are There Specific Optima? In: Lipids in Aquatic Ecosystems (eds. Kainz, M., Brett, M.T. \& Arts, M.T.). Springer New York, New York, NY, pp. 147-178.

Allendorf, F.W. \& Lundquist, L.L. (2003). Introduction: Population Biology, Evolution, and Control of Invasive Species. Conserv. Biol., 17, 24-30.

Anderson, T.R. \& Hessen, D.O. (2005). Threshold elemental ratios for carbon versus phosphorus limitation in Daphnia. Freshw. Biol., 50, 2063-2075.

Andrade, M.C.B. \& Roitberg, B.D. (1995). Rapid response to intraclonal selection in the pea aphid (Acyrthosiphon pisum). Evol. Ecol., 9, 397-410.

Archambeault, S.L., Durston, D.J., Wan, A., El-Sabaawi, R.W., Matthews, B. \& Peichel, C.L. (2020). Phosphorus limitation does not drive loss of bony lateral plates in freshwater stickleback (Gasterosteus aculeatus). Evolution, 74, 2088-2104.

Arnold, K.H., Shreeve, R.S., Atkinson, A. \& Clarke, A. (2004). Growth rates of Antarctic krill, Euphausia superba: Comparison of the instantaneous growth rate method with nitrogen and phosphorus stoichiometry. Limnol. Oceanogr., 49, 2152-2161.

Arthur, P. F., and Herd, R. M. (2005). Efficiency of feed utilisation by livestock - Implications and benefits of genetic improvement. Can. J. Anim. Sci., 85, 281-290.

Asselman, J., De Coninck, D.I.M., Vandegehuchte, M.B., Jansen, M., Decaestecker, E., De Meester, L., et al. (2015). Global cytosine methylation in Daphnia magna depends on genotype, environment, and their interaction. Environ. Toxicol. Chem., 34, 1056-1061.

Bakker, E. G., Stahl, E. A., Toomajian, C., Nordborg, M., Kreitman, M., and Bergelson, J. (2006). Distribution of genetic variation within and among local populations of Arabidopsis thaliana over its species range: population structure of Arabidopsis. Mol. Ecol., 15, 1405-1418.

Barbour, M. A., Rodriguez-Cabal, M. A., Wu, E. T., Julkunen-Tiitto, R., Ritland, C. E., Miscampbell, A. E., et al. (2015). Multiple plant traits shape the genetic basis of herbivore community assembly. Funct. Ecol., 29, 995-1006.

Barrett, R.D.H. \& Schluter, D. (2008). Adaptation from standing genetic variation. Trends Ecol. Evol., 23, 38-44.

Barrick, J.E. \& Lenski, R.E. (2013). Genome dynamics during experimental evolution. Nat. Rev. Genet., 14, 827-839.

Bassar, R. D., Marshall, M. C., López-Sepulcre, A., Zandonà, E., Auer, S. K., Travis, J., et al. (2010). Local adaptation in Trinidadian guppies alters ecosystem processes. Proc. Natl. Acad. Sci. U.S.A., 107, 3616-3621.

Bates, D., Maechler, M., Bolker, B. \& Walker, S. (2015). Fitting linear mixed-effects models using Ime4. J. Stat. Softw., 67, 1-48.

Becks, L. \& Agrawal, A.F. (2010). Higher rates of sex evolve in spatially heterogeneous environments. Nature, 468, 89-92.

Becks, L. \& Agrawal, A.F. (2012). The evolution of sex is favoured during adaptation to new environments. PLoS Biol., 10, e1001317.

Becks, L. \& Agrawal, A.F. (2013). Higher rates of sex evolve under K-selection. J. Evol. Biol., 26, 900905.

Benton, T.G., Solan, M., Travis, J.M.J. \& Sait, S.M. (2007). Microcosm experiments can inform global ecological problems. Trends Ecol. Evol., 22, 516-521. 
Birky, C.W. \& Gilbert, J.J. (1971). Parthenogenesis in Rotifers: The Control of Sexual and Asexual Reproduction. Am. Zool., 11, 245-266.

Bland, M. (2015). Estimating mean and standard deviation from the sample size, three quartiles, minimum, and maximum. Int. J. Stats. Med., Res. 4, 57-64.

Boersma, M. (2000). The nutritional quality of P-limited algae for Daphnia. Limnol. Oceanogr., 45, 1157-1161.

Boersma, M., Aberle, N., Hantzsche, F.M., Schoo, K.L., Wiltshire, K.H. \& Malzahn, A.M. (2008). Nutritional Limitation Travels up the Food Chain. Int. Rev. Hydrobiol., 93, 479-488.

Bolker, B., and R Development Core Team. (2017). bbmle: tools for general maximum likelihood estimation. R package version 1.0.20. Available via https://CRAN.R-project.org/package=bbmle.

Bolnick, D.I., Amarasekare, P., Araújo, M.S., Bürger, R., Levine, J.M., Novak, M., et al. (2011). Why intraspecific trait variation matters in community ecology. Trends Ecol. Evol., 26, 183-192.

Bonduriansky, R. \& Day, T. (2009). Nongenetic Inheritance and Its Evolutionary Implications. Annu. Rev. Ecol. Evol. Syst., 40, 103-125.

Bonduriansky, R. \& Day, T. (2018). Extended Heredity: A New Understanding of Inheritance and Evolution. Princeton University Press.

Botero, C.A., Weissing, F.J., Wright, J. \& Rubenstein, D.R. (2015). Evolutionary tipping points in the capacity to adapt to environmental change. Proc. Natl. Acad. Sci. U. S. A., 112, 184-189.

Bradshaw, A.D. (1965). Evolutionary Significance of Phenotypic Plasticity in Plants. In: Advances in Genetics (eds. Caspari, E.W. \& Thoday, J.M.). Academic Press, pp. 115-155.

Branco, P., Egas, M., Elser, J.J. \& Huisman, J. (2018). Eco-Evolutionary Dynamics of Ecological Stoichiometry in Plankton Communities. Am. Nat., 192, E1-E20.

Branco, P., Stomp, M., Egas, M. \& Huisman, J. (2010). Evolution of nutrient uptake reveals a trade-off in the ecological stoichiometry of plant-herbivore interactions. Am. Nat., 176, E162-76.

Bunting, S. \& Van Emden, H.F. (1980). Rapid response to selection for increased esterase activity on small populations of an apomictic clone of Myzus persicae. Nature, 285, 502-503.

Burge, D.R.L., Edlund, M.B. \& Frisch, D. (2018). Paleolimnology and resurrection ecology: The future of reconstructing the past. Evol. Appl., 11, 42-59.

Burian, A., Grosse, J., Winder, M. \& Boschker, H.T.S. (2018). Nutrient deficiencies and the restriction of compensatory mechanisms in copepods. Funct. Ecol., 32, 636-647.

Burian, A., Nielsen, J.M. \& Winder, M. (2020). Food quantity-quality interactions and their impact on consumer behavior and trophic transfer. Ecol. Monogr., 90.

Burnham, K.P. \& Anderson, D.R. (2004). Multimodel Inference: Understanding AIC and BIC in Model Selection. Sociol. Methods Res., 33, 261-304.

Burnham, K. P., Anderson, D. R., and Burnham, K. P. (2002). Model Selection and Multimodel Inference: A Practical Information-Theoretic Approach, 2nd Edn. New York, NY: Springer.Butlin, R. (2002). Evolution of sex: The costs and benefits of sex: new insights from old asexual lineages. Nat. Rev. Genet., 3, 311-317.

Canty A, Ripley BD (2020). boot: Bootstrap R (S-Plus) Functions. R package version 1.3-25.

Carroll, S.P., Hendry, A.P., Reznick, D.N. \& Fox, C.W. (2007). Evolution on ecological time-scales. Funct. Ecol., 21, 387-393.

Cease, A.J., Fay, M., Elser, J.J. \& Harrison, J.F. (2016). Dietary phosphate affects food selection, post-ingestive phosphorus fate, and performance of a polyphagous herbivore. J. Exp. Biol., 219, 64-72.

Chan, Y.F., Marks, M.E., Jones, F.C., Villarreal, G., Jr, Shapiro, M.D., Brady, S.D., et al. (2010). Adaptive evolution of pelvic reduction in sticklebacks by recurrent deletion of a Pitx1 enhancer. Science, 327, 302-305. 
Chowdhury, P.R. \& Jeyasingh, P.D. (2016). Differences in phosphorus use between ancient and extant Daphnia genotypes alters algal stoichiometry and abundance. Inland Waters, 6, 165-172.

Chowdhury, P. R., Lopez, J. A., Weider, L. J., Colbourne, J. K., and Jeyasingh, P. D. (2014).

Functional genomics of intraspecific variation in carbon and phosphorus kinetics in Daphnia: Sources of variation in carbon and phosphorus use. J. Exp. Zool., 321, 387-398.

Colicchio, J.M. \& Herman, J. (2020). Empirical patterns of environmental variation favor adaptive transgenerational plasticity. Ecol. Evol., 10, 1648-1665.

Colina, M., Calliari, D., Carballo, C. \& Kruk, C. (2016). A trait-based approach to summarize zooplankton-phytoplankton interactions in freshwaters. Hydrobiologia, 767, 221-233.

Collins, M., Knutti, R., Arblaster, J., Dufresne, J., Fichefet, T., Friedlingstein, P., et al. (2013). Longterm climate change: Projections, commitments and irreversibility. In: Climate change 2013: The physical science basis. Contribution of Working Group I to the Fifth Assessment Report of the Intergovernmental Panel on Climate Change (eds Stocker, T.F., Qin, D., Plattner, G., Tignor, M., Allen, S.K., Boschung, J., Nauels, A., Xia, Y., Bex, V., \& Midgley, P. M.)., Cambridge University Press, Cambridge, United Kingdom and New York, NY.

Conover, D.O., Duffy, T.A. \& Hice, L.A. (2009). The covariance between genetic and environmental influences across ecological gradients: reassessing the evolutionary significance of countergradient and cogradient variation. Ann. N. Y. Acad. Sci., 1168, 100-129.

Cordell, D., Drangert, J.-O. \& White, S. (2009). The story of phosphorus: Global food security and food for thought. Glob. Environ. Change, 19, 292-305.

Crutsinger, G. M., Collins, M. D., Fordyce, J. A., Gompert, Z., Nice, C. C., and Sanders, N. J. (2006). Plant genotypic diversity predicts community structure and governs an ecosystem process. Science, 313, 966-968.

Crutsinger, G. M., Rodriguez-Cabal, M. A., Roddy, A. B., Peay, K. G., Bastow, J. L., Kidder, A. G., et al. (2014a). Genetic variation within a dominant shrub structures green and brown community assemblages. Ecology, 95, 387-398.

Crutsinger, G. M., Rudman, S. M., Rodriguez-Cabal, M. A., McKown, A. D., Sato, T., MacDonald, A. M., et al. (2014b). Testing a 'genes-to-ecosystems' approach to understanding aquatic-terrestrial linkages. Mol. Ecol., 23, 5888-5903.

Crutsinger, G. M., Sanders, N. J., and Classen, A. T. (2009). Comparing intra- and inter-specific effects on litter decomposition in an old-field ecosystem. Basic Appl. Ecol., 10, 535-543.

Dalton, C. M., El-Sabaawi, R. W., Honeyfield, D. C., Auer, S. K., Reznick, D. N., and Flecker, A. S. (2017). The influence of dietary and whole-body nutrient content on the excretion of a vertebrate consumer. PLoS One, 12:e0187931.

Darchambeau, F. (2005). Filtration and digestion responses of an elementally homeostatic consumer to changes in food quality: a predictive model. Oikos, 111, 322-336.

Darchambeau, F., Faerøvig, P.J. \& Hessen, D.O. (2003). How Daphnia copes with excess carbon in its food. Oecologia, 136, 336-346.

Daugaard, U., Petchey, O.L. \& Pennekamp, F. (2019). Warming can destabilize predator-prey interactions by shifting the functional response from Type III to Type II. J Anim Ecol., 88, 1575-1586.

Day, T. \& Bonduriansky, R. (2011). A unified approach to the evolutionary consequences of genetic and nongenetic inheritance. Am. Nat., 178, E18-36.

Declerck, S.A.J., Malo, A.R., Diehl, S., Waasdorp, D., Lemmen, K.D., Proios, K., et al. (2015). Rapid adaptation of herbivore consumers to nutrient limitation: eco-evolutionary feedbacks to population demography and resource control. Ecol. Lett., 18, 553-562.

Declerck, S.A.J. \& Papakostas, S. (2017). Monogonont rotifers as model systems for the study of micro-evolutionary adaptation and its eco-evolutionary implications. Hydrobiologia, 796, 131-144. 
De Meester, L. (1996). Local genetic differentiation and adaptation in freshwater zooplankton populations: Patterns and processes. Écoscience, 3, 385-399.

De Meester, L., Gómez, A., Okamura, B. \& Schwenk, K. (2002). The Monopolization Hypothesis and the dispersal-gene flow paradox in aquatic organisms. Acta Oecol., 23, 121-135.

DeMott, W.R., Gulati, R.D. \& Siewertsen, K. (1998). Effects of phosphorus-deficient diets on the carbon and phosphorus balance of Daphnia magna. Limnol. Oceanogr., 43, 1147-1161.

DeMott, W. R., Pape, B. J., and Tessier, A. J. (2004). Patterns and sources of variation in Daphnia phosphorus content in nature. Aquat. Ecol., 38, 433-440.

Des Roches, S., Post, D.M., Turley, N.E., Bailey, J.K., Hendry, A.P., Kinnison, M.T., et al. (2018). The ecological importance of intraspecific variation. Nat Ecol Evol, 2, 57-64.

de Verdal, H., Narcy, A., Bastianelli, D., Même, N., Urvoix, S., Collin, A., et al. (2013). Genetic variability of metabolic characteristics in chickens selected for their ability to digest wheat. J. Anim. Sci., 91, 2605-2615.

Dick, J.T.A., Alexander, M.E., Jeschke, J.M., Ricciardi, A., Maclsaac, H.J., Robinson, T.B., et al. (2014). Advancing impact prediction and hypothesis testing in invasion ecology using a comparative functional response approach. Biol. Invasions, 16, 735-753.

Dickman, E.M., Newell, J.M., González, M.J. \& Vanni, M.J. (2008). Light, nutrients, and food-chain length constrain planktonic energy transfer efficiency across multiple trophic levels. Proc. Natl. Acad. Sci. U. S. A., 105, 18408-18412.

Diehl, S. (2003). The evolution and maintenance of omnivory: Dynamic constraints and the role of food quality. Ecology, 84, 2557-2567.

Dinh Van, K., Janssens, L., Debecker, S., De Jonge, M., Lambret, P., Nilsson-Örtman, V., et al. (2013). Susceptibility to a metal under global warming is shaped by thermal adaptation along a latitudinal gradient. Glob. Change Biol., 19, 2625-2633.

Dukić, M., Berner, D., Haag, C.R. \& Ebert, D. (2019). How clonal are clones? A quest for loss of heterozygosity during asexual reproduction in Daphnia magna. J. Evol. Biol., 32, 619-628.

Eckert, C.G., Samis, K.E. \& Lougheed, S.C. (2008). Genetic variation across species' geographical ranges: the central-marginal hypothesis and beyond. Mol. Ecol., 17, 1170-1188.

Efron, B. (1987). Better Bootstrap Confidence Intervals. JASA, 82, 171-185.

von Elert, E., Martin-Creuzburg, D. \& Le Coz, J.R. (2003). Absence of sterols constrains carbon transfer between cyanobacteria and a freshwater herbivore (Daphnia galeata). Proc. Biol. Sci., 270, 1209-1214.

Ehrlich, P. R., and Raven, P. H. (1969). Differentiation of populations. Science, 165, 1228-1232.

El-Sabaawi, R.W., Warbanski, M.L., Rudman, S.M., Hovel, R. \& Matthews, B. (2016). Investment in boney defensive traits alters organismal stoichiometry and excretion in fish. Oecologia, 181, 12091220.

Elser, J. (2006). Biological stoichiometry: a chemical bridge between ecosystem ecology and evolutionary biology. Am. Nat., 168 Suppl 6, S25-35.

Elser, J. J. (2003). Biological stoichiometry: a theoretical framework connecting ecosystem ecology, evolution, and biochemistry for application in astrobiology. Int. J. Astrobiol., 2, 185-193.

Elser, J.J., Acharya, K., Kyle, M., Cotner, J., Makino, W., Markow, T., et al. (2003). Growth ratestoichiometry couplings in diverse biota. Ecol. Lett., 6, 936-943.

Elser, J.J., Andersen, T., Baron, J.S., Bergström, A.-K., Jansson, M., Kyle, M., et al. (2009). Shifts in lake N:P stoichiometry and nutrient limitation driven by atmospheric nitrogen deposition. Science, 326, 835-837. 
Elser, J.J., Bracken, M.E.S., Cleland, E.E., Gruner, D.S., Harpole, W.S., Hillebrand, H., et al. (2007a). Global analysis of nitrogen and phosphorus limitation of primary producers in freshwater, marine and terrestrial ecosystems. Ecol. Lett., 10, 1135-1142.

Elser, J.J., Dobberfuhl, D.R., MacKay, N.A. \& Schampel, J.H. (1996). Organism size, life history, and N: P stoichiometry: toward a unified view of cellular and ecosystem processes. Bioscience, 46, 674684.

Elser, J.J., Elser, M.M., MacKay, N.A. \& Carpenter, S.R. (1988). Zooplankton-mediated transitions between $\mathrm{N}$ - and P-limited algal growth1. Limnol. Oceanogr., 33, 1-14.

Elser, J. J., Fagan, W. F., Denno, R. F., Dobberfuhl, D. R., Folarin, A., Huberty, A., et al. (2000a). Nutritional constraints in terrestrial and freshwater food webs. Nature, 408, 578-580.

Elser, J.J., Hayakawa, K. \& Urabe, J. (2001). Nutrient Limitation Reduces Food Quality for Zooplankton: Daphnia Response to Seston Phosphorus Enrichment. Ecology, 82, 898-903.

Elser, J. J., Kyle, M. M., Smith, M. S., and Nagy, J. D. (2007b). Biological stoichiometry in human cancer. PLoS One, 2:e1028.

Elser, J.J., Sterner, R.W., Gorokhova, E., Fagan, W.F., Markow, T.A., Cotner, J.B., et al. (2000b). Biological stoichiometry from genes to ecosystems. Ecol. Lett., 3, 540-550.

Elser, J.J. \& Urabe, J. (1999). The stoichiometry of consumer-driven nutrient recycling: theory, observations, and consequences. Ecology, 80, 735-751.

Elser, J.J., Watts, J., Schampel, J.H. \& Farmer, J. (2006). Early Cambrian food webs on a trophic knife-edge? A hypothesis and preliminary data from a modern stromatolite-based ecosystem. Ecol. Lett., 9, 295-303.

Elser, J.J., O'Brien, W.J., Dobberfuhl, D.R. \& Dowling, T.E. (2000c). The evolution of ecosystem processes: growth rate and elemental stoichiometry of a key herbivore in temperate and arctic habitats. J. Evol. Biol., 13, 845-853.

Englund, G., Ohlund, G., Hein, C.L. \& Diehl, S. (2011). Temperature dependence of the functional response. Ecol. Lett., 14, 914-921.

Espeset, A., Kobiela, M. E., Sikkink, K. L., Pan, T., Roy, C., and Snell-Rood, E. C. (2019). Anthropogenic increases in nutrients alter sexual selection dynamics: a case study in butterflies. Behav. Ecol., 30, 598-608.

Excoffier, L., Foll, M. \& Petit, R.J. (2009). Genetic Consequences of Range Expansions. Annu. Rev. Ecol. Evol. Syst., 40, 481-501.

Ferrão-Filho, A.D.S., Tessier, A.J. \& DeMott, W.R. (2007). Sensitivity of herbivorous zooplankton to phosphorus-deficient diets: Testing stoichiometric theory and the growth rate hypothesis. Limnol. Oceanogr., 52, 407-415.

Fink, P. \& Von Elert, E. (2006). Physiological responses to stoichiometric constraints: nutrient limitation and compensatory feeding in a freshwater snail. Oikos, 115, 484-494.

Flores, L., Larrañaga, A. \& Elosegi, A. (2014). Compensatory feeding of a stream detritivore alleviates the effects of poor food quality when enough food is supplied. Freshw. Sci., 33, 134-141.

Fischer, J. \& Lindenmayer, D.B. (2007). Landscape modification and habitat fragmentation: a synthesis. Glob. Ecol. Biogeogr., 16, 265-280.

Flores, L., Larrañaga, A. \& Elosegi, A. (2014). Compensatory feeding of a stream detritivore alleviates the effects of poor food quality when enough food is supplied. Freshw. Sci., 33, 134-141.

Fournier, A., Penone, C., Pennino, M.G. \& Courchamp, F. (2019). Predicting future invaders and future invasions. Proc. Natl. Acad. Sci. U. S. A., 116, 7905-7910.

Fox, J. \& Weisberg, S. (2011). An R Companion to Applied Regression, 2nd edn. Sage, Thousand Oaks, CA. 
Franch-Gras, L., García-Roger, E.M., Serra, M. \& José Carmona, M. (2017). Adaptation in response to environmental unpredictability. Proc. Biol. Sci., 284.

Frisch, D., Morton, P.K., Chowdhury, P.R., Culver, B.W., Colbourne, J.K., Weider, L.J., et al. (2014). A millennial-scale chronicle of evolutionary responses to cultural eutrophication in Daphnia. Ecol. Lett., 17, 360-368.

Frost, P.C., Benstead, J.P., Cross, W.F., Hillebrand, H., Larson, J.H., Xenopoulos, M.A., et al. (2006). Threshold elemental ratios of carbon and phosphorus in aquatic consumers. Ecol. Lett., 9, 774-779.

Fryxell, D.C., Wood, Z.T., Robinson, R., Kinnison, M.T. \& Palkovacs, E.P. (2019). Eco-evolutionary feedbacks link prey adaptation to predator performance. Biol. Lett., 15, 20190626.

Fussmann, G.F. (2003). Evolution as a critical component of plankton dynamics. Proc. R. Soc. Lond. B Biol. Sci., 270, 1015-1022.

Fussmann, G.F., Loreau, M. \& Abrams, P.A. (2007). Eco-evolutionary dynamics of communities and ecosystems. Funct. Ecol., 21, 465-477.

Fussmann, G.F., Weithoff, G. \& Yoshida, T. (2005). A DIRECT, EXPERIMENTAL TEST OF RESOURCE VS. CONSUMER DEPENDENCE. Ecology, 86, 2924-2930.

Futuyma, D.J. (2010). Evolutionary constraint and ecological consequences. Evolution, 64, 18651884.

Galloway, J.N., Dentener, F.J., Capone, D.G., Boyer, E.W., Howarth, R.W., Seitzinger, S.P., et al. (2004). Nitrogen Cycles: Past, Present, and Future. Biogeochemistry, 70, 153-226.

Galloway, J.N., Townsend, A.R., Erisman, J.W., Bekunda, M., Cai, Z., Freney, J.R., et al. (2008).

Transformation of the nitrogen cycle: recent trends, questions, and potential solutions. Science, 320, 889-892.

Garcia-Roger, E.M., Jose Carmona, M. \& Serra, M. (2017). Modes, mechanisms and evidence of bet hedging in rotifer diapause traits. Hydrobiologia, 796, 223-233.

Gascoigne, J.C. \& Lipcius, R.N. (2004). Allee Effects Driven by Predation. J. Appl. Ecol., 41, 801-810.

Gillis, M.K. \& Walsh, M.R. (2019). Individual variation in plasticity dulls transgenerational responses to stress. Funct. Ecol., 33, 1993-2002.

Gillooly, J.F., Allen, A.P., Brown, J.H., Elser, J.J., Martinez del Rio, C., Savage, V.M., et al. (2005). The metabolic basis of whole-organism RNA and phosphorus content. Proc. Natl. Acad. Sci. U.S.A., 102, 11923-11927.

Golani, D., Azzurro, E., Corsini-Foka, M., Falautano, M., Andaloro, F. \& Bernardi, G. (2007). Genetic bottlenecks and successful biological invasions: the case of a recent Lessepsian migrant. Biol. Lett., $3,541-545$.

Gomes, C., Sousa, R., Mendes, T., Borges, R., Vilares, P., Vasconcelos, V., et al. (2016). Low Genetic Diversity and High Invasion Success of Corbicula fluminea (Bivalvia, Corbiculidae) (Müller, 1774) in Portugal. PLoS One, 11, e0158108.

Gómez, A. \& Carvalho, G.R. (2000). Sex, parthenogenesis and genetic structure of rotifers: microsatellite analysis of contemporary and resting egg bank populations. Mol. Ecol., 9, 203-214.

González, A.L., Dézerald, O., Marquet, P.A., Romero, G.Q. \& Srivastava, D.S. (2017). The Multidimensional Stoichiometric Niche. Frontiers in Ecology and Evolution, 5, 110.

González, A. L., Fariña, J. M., Kay, A. D., Pinto, R., and Marquet, P. A. (2011). Exploring patterns and mechanisms of interspecific and intraspecific variation in body elemental composition of desert consumers. Oikos, 120, 1247-1255.

González, A.L., Romero, G.Q. \& Srivastava, D.S. (2014). Detrital nutrient content determines growth rate and elemental composition of bromeliad-dwelling insects. Freshw. Biol., 59, 737-747. 
Gorokhova, E., Dowling, T.A., Weider, L.J., Crease, T. \& Elser, J.J. (2002). Functional and ecological significance of rDNA IGS variation in a clonal organism under divergent selection for production rate. Proc. R. Soc. Lond. B, 269, 2373-2379.

Gribble, K.E. \& Welch, D.B.M. (2017). Genome-wide transcriptomics of aging in the rotifer Brachionus manjavacas, an emerging model system. BMC Genomics, 18, 217.

Guijun, Y., Boqiang, Q., Xiangming, T., Zhijun, G., Chunni, Z., Hua, Z., et al. (2012). Contrasting zooplankton communities of two bays of the large, shallow, eutrophic Lake Taihu, China: Their relationship to environmental factors. J. Great Lakes Res., 38, 299-308.

Güsewell, S., Jakobs, G., and Weber, E. (2006). Native and introduced populations of Solidago gigantea differ in shoot production but not in leaf traits or litter decomposition. Funct. Ecol., 20, 575-584.

Haafke, J., Abou Chakra, M. \& Becks, L. (2016). Eco-evolutionary feedback promotes Red Queen dynamics and selects for sex in predator populations. Evolution, 70, 641-652.

Hadfield, J. (2010). MCMC methods for multi-response generalized linear mixed models: the MCMCglmm R package. J. Stat. Softw., 33, 1-22.

Haileselasie, T.H., Mergeay, J., Vanoverbeke, J., Orsini, L. \& De Meester, L. (2018). Founder effects determine the genetic structure of the water flea Daphnia in Ethiopian reservoirs : Founder effects in zooplankton populations. Limnol. Oceanogr., 63, 915-926.

Hairston, N.G., Jr, Ellner, S.P., Geber, M.A., Yoshida, T. \& Fox, J.A. (2005). Rapid evolution and the convergence of ecological and evolutionary time. Ecol. Lett., 8, 1114-1127

Hairston, N.G., Perry, L.J., Jr., Bohonak, A.J., Fellows, M.Q., Kearns, C.M. \& Engstrom, D.R. (1999). Population biology of a failed invasion: Paleolimnology of Daphnia exilis in upstate New York. Limnol. Oceanogr., 44, 477-486.

Hall, S.R. (2009). Stoichiometrically explicit food webs: feedbacks between resource supply, elemental constraints, and species diversity. Annu. Rev. Ecol. Evol. Syst., 40, 503-528.

Hall, S.R., Shurin, J.B., Diehl, S. \& Nisbet, R.M. (2007). Food quality, nutrient limitation of secondary production, and the strength of trophic cascades. Oikos, 116, 1128-1143.

Hansen, T. F., Pélabon, C., and Houle, D. (2011). Heritability is not evolvability. Evol. Biol., 38, 258277.

Harmon, L. J., Matthews, B., Des Roches, S., Chase, J. M., Shurin, J. B., and Schluter, D. (2009). Evolutionary diversification in stickleback affects ecosystem functioning. Nature, 458, 1167-1170.

Harrison, X.A. (2014). Using observation-level random effects to model overdispersion in count data in ecology and evolution. PeerJ, 2, e616.

Hartley, C.J., Newcomb, R.D., Russell, R.J., Yong, C.G., Stevens, J.R., Yeates, D.K., et al. (2006). Amplification of DNA from preserved specimens shows blowflies were preadapted for the rapid evolution of insecticide resistance. Proc. Natl. Acad. Sci. U. S. A., 103, 8757-8762.

Hartwich, M., Martin-Creuzburg, D., Rothhaupt, K.-O. \& Wacker, A. (2012). Oligotrophication of a large, deep lake alters food quantity and quality constraints at the primary producer-consumer interface. Oikos, 121, 1702-1712.

Hassett, R.P., Cardinale, B., Stabler, L.B. \& Elser, J.J. (1997). Ecological stoichiometry of N and P in pelagic ecosystems: Comparison of lakes and oceans with emphasis on the zooplanktonphytoplankton interaction. Limnol. Oceanogr., 42, 648-662.

He, X. \& Wang, W.-X. (2007). Kinetics of phosphorus in Daphnia at different food concentrations and carbon:phosphorus ratios. Limnol. Oceanogr., 52, 395-406.

Hedges, L. V., Gurevitch, J., and Curtis, P. S. (1999). The meta-analysis of response ratios in experimental ecology. Ecology, 80, 1150-1156.

Hendry, A.P. (2016). Eco-Evolutionary Dynamics. Princeton University Press, Princeton, NJ. 
Hendry, A.P. \& Kinnison, M.T. (1999). PERSPECTIVE: THE PACE OF MODERN LIFE: MEASURING RATES OF CONTEMPORARY MICROEVOLUTION. Evolution, 53, 1637-1653.

Hereford, J., Hansen, T. F., and Houle, D. (2004). Comparing strengths of directional selection: how strong is strong? Evolution, 58, 2133-2143.

Herman, J.J., Spencer, H.G., Donohue, K. \& Sultan, S.E. (2014). How stable "should" epigenetic modifications be? Insights from adaptive plasticity and bet hedging. Evolution, 68, 632-643.

Herman, J.J. \& Sultan, S.E. (2016). DNA methylation mediates genetic variation for adaptive transgenerational plasticity. Proc. Biol. Sci., 283.

Hermisson, J. \& Pennings, P.S. (2005). Soft sweeps: molecular population genetics of adaptation from standing genetic variation. Genetics, 169, 2335-2352.

Hessen, D.O. \& Anderson, T.R. (2008). Excess carbon in aquatic organisms and ecosystems: Physiological, ecological, and evolutionary implications. Limnol. Oceanogr., 53, 1685-1696.

Hessen, D.O., van Donk, E. \& Gulati, R. (2005). Seasonal seston stoichiometry: effects on zooplankton in cyanobacteria-dominated lakes. J. Plankton Res., 27, 449-460.

Higgins, J. P., and Green, S. eds. (2008). Cochrane Handbook for Systematic Reviews of Interventions. Chichester: John Wiley and Sons, Ltd.

Hillebrand, H., Borer, E.T., Bracken, M.E.S., Cardinale, B.J., Cebrian, J., Cleland, E.E., et al. (2009). Herbivore metabolism and stoichiometry each constrain herbivory at different organizational scales across ecosystems. Ecol. Lett., 12, 516-527.

Ho, E.K.H., Macrae, F., Latta, L.C., Benner, M.J., Sun, C., Ebert, D., et al. (2019). Intraspecific Variation in Microsatellite Mutation Profiles in Daphnia magna. Mol. Biol. Evol., 36, 1942-1954.

Hoekstra, H.E., Hirschmann, R.J., Bundey, R.A., Insel, P.A. \& Crossland, J.P. (2006). A single amino acid mutation contributes to adaptive beach mouse color pattern. Science, 313, 101-104.

Holling, C.S. (1959) Some Characteristics of Simple Types of Predation and Parasitism. Can. Entomol., 91, 385-398.

Hollingsworth, M.L. \& Bailey, J.P. (2000). Evidence for massive clonal growth in the invasive weed Fallopia japonica (Japanese Knotweed). Bot. J. Linn. Soc., 133, 463-472.

Hood, J.M. \& Sterner, R.W. (2014). Carbon and phosphorus linkages in Daphnia growth are determined by growth rate, not species or diet. Funct. Ecol., 28, 1156-1165.

Hou, E., Luo, Y., Kuang, Y., Chen, C., Lu, X., Jiang, L., et al. (2020). Global meta-analysis shows pervasive phosphorus limitation of aboveground plant production in natural terrestrial ecosystems. Nat. Commun., 11, 637.

Houri-Ze'evi, L., Korem, Y., Sheftel, H., Faigenbloom, L., Toker, I.A., Dagan, Y., et al. (2016). A Tunable Mechanism Determines the Duration of the Transgenerational Small RNA Inheritance in C. elegans. Cell, 165, 88-99.

Houri-Ze'evi, L. \& Rechavi, O. (2017). A Matter of Time: Small RNAs Regulate the Duration of Epigenetic Inheritance. Trends Genet., 33, 46-57.

Hu, J. \& Barrett, R.D.H. (2017). Epigenetics in natural animal populations. J. Evol. Biol., 30, 16121632.

Hughes, A.R., Inouye, B.D., Johnson, M.T.J., Underwood, N. \& Vellend, M. (2008). Ecological consequences of genetic diversity. Ecol. Lett., 11, 609-623.

Hughes, A. R., Stachowicz, J. J., and Williams, S. L. (2009). Morphological and physiological variation among seagrass (Zostera marina) genotypes. Oecologia, 159, 725-733.

lannino, A., Vosshage, A.T.L. \& Weitere, M. (2019). High nutrient availability leads to weaker topdown control of stream periphyton: Compensatory feeding in Ancylus fluviatilis. Freshw. Biol.

Jablonka, E. \& Raz, G. (2009). Transgenerational epigenetic inheritance: prevalence, mechanisms, and implications for the study of heredity and evolution. Q. Rev. Biol., 84, 131-176 
Jain, S. K., and Bradshaw, A. D. (1966). Evolutionary divergence among adjacent plant populations I. The evidence and its theoretical analysis. Heredity, 21, 407-441.

Jensen, T.C., Anderson, T.R., Daufresne, M. \& Hessen, D.O. (2006). Does excess carbon affect respiration of the rotifer Brachionus calyciflorus Pallas? Freshw. Biol., 51, 2320-2333.

Jensen, T.C. \& Hessen, D.O. (2007). Does excess dietary carbon affect respiration of Daphnia? Oecologia, 152, 191-200.

Jeppesen, E., Jensen, J.P. \& Søndergaard, M. (2002). Response of phytoplankton, zooplankton, and fish to re-oligotrophication: An 11 year study of 23 Danish lakes. Aquat. Ecosyst. Health Manag., 5, 31-43.

Jeppesen, E., Sondergaard, M., Jensen, J.P., Havens, K.E., Anneville, O., Carvalho, L., et al. (2005). Lake responses to reduced nutrient loading - an analysis of contemporary long-term data from 35 case studies. Freshw. Biol., 50, 1747-1771.

Jeremias, G., Barbosa, J., Marques, S.M., Asselman, J., Gonçalves, F.J.M. \& Pereira, J.L. (2018). Synthesizing the role of epigenetics in the response and adaptation of species to climate change in freshwater ecosystems. Mol. Ecol., 27, 2790-2806.

Jeschke, J.M., Kopp, M. \& Tollrian, R. (2002). PREDATOR FUNCTIONAL RESPONSES: DISCRIMINATING BETWEEN HANDLING AND DIGESTING PREY. ECol. Monogr., 72, 95-112.

Jeschke, J.M., Kopp, M. \& Tollrian, R. (2004). Consumer-food systems: why type I functional responses are exclusive to filter feeders. Biol. Rev. Camb. Philos. Soc., 79, 337-349.

Jessup, C.M., Kassen, R., Forde, S.E., Kerr, B., Buckling, A., Rainey, P.B., et al. (2004). Big questions, small worlds: microbial model systems in ecology. Trends Ecol. Evol., 19, 189-197.

Jeyasingh, P. D., Chowdhury, P. R., Wojewodzic, M. W., Frisch, D., Hessen, D. O., and Weider, L. J. (2015). Phosphorus use and excretion varies with ploidy level in Daphnia. J. Plankton Res., fbv095.

Jeyasingh, P.D., Cothran, R.D. \& Tobler, M. (2014). Testing the ecological consequences of evolutionary change using elements. Ecol. Evol., 4, 528-538.

Jeyasingh, P. D., and Weider, L. J. (2007). Fundamental links between genes and elements: evolutionary implications of ecological stoichiometry. Mol. Ecol., 16, 4649-4661.

Jeyasingh, P. D., Weider, L. J., and Sterner, R. W. (2009). Genetically-based trade-offs in response to stoichiometric food quality influence competition in a keystone aquatic herbivore. Ecol. Lett., 12, 1229-1237.

Jochum, M., Barnes, A.D., Ott, D., Lang, B., Klarner, B., Farajallah, A., et al. (2017). Decreasing Stoichiometric Resource Quality Drives Compensatory Feeding across Trophic Levels in Tropical Litter Invertebrate Communities. Am. Nat., 190, 131-143.

Johnson, M. T. J., Agrawal, A. A., Maron, J. L., and Salminen, J.-P. (2009). Heritability, covariation and natural selection on 24 traits of common evening primrose (Oenothera biennis) from a field experiment. J. Evol. Biol., 22, 1295-1307.

Joyce, G. F. (1989). RNA evolution and the origins of life. Nature, 338, 217-224.

Judson, O.P. \& Normark, B.B. (1996). Ancient asexual scandals. Trends Ecol. Evol., 11, 41-46.

Jumars, P.A. (2000). Animal Guts as Ideal Chemical Reactors: Maximizing Absorption Rates. Am. Nat., 155, 527-543.

J. Marshall, D. \& Uller, T. (2007). When is a maternal effect adaptive? Oikos, 116, 1957-1963.

Kalinkat, G., Schneider, F.D., Digel, C., Guill, C., Rall, B.C. \& Brose, U. (2013). Body masses, functional responses and predator-prey stability. Ecol. Lett., 16, 1126-1134.

Kauler, P. \& Enesco, H.E. (2011). The effect of temperature on life history parameters and cost of reproduction in the rotifer Brachionus calyciflorus. J. Freshw. Ecol., 26, 399-408.

Kawecki, T.J. \& Ebert, D. (2004). Conceptual issues in local adaptation. Ecol. Lett., 7, 1225-1241. 
Kawecki, T.J., Lenski, R.E., Ebert, D., Hollis, B., Olivieri, I. \& Whitlock, M.C. (2012). Experimental evolution. Trends Ecol. Evol., 27, 547-560.

Kay, A.D., Ashton, I.W., Gorokhova, E., Kerkhoff, A.J., Liess, A. \& Litchman, E. (2005). Toward a stoichiometric framework for evolutionary biology. Oikos, 109, 6-17.

Keitt, T.H. (2017). odeintr: C++ ODE Solvers Compiled on-Demand. R package version 1.7.1. https://CRAN.R-project.org/package=odeintr

Kielland, $\varnothing$. N., Bech, C. \& Einum, S. (2017). No evidence for thermal transgenerational plasticity in metabolism when minimizing the potential for confounding effects. Proc. Biol. Sci., 284.

Kilham, S.S., Kreeger, D.A., Lynn, S.G., Goulden, C.E. \& Herrera, L. (1998). COMBO: a defined freshwater culture medium for algae and zooplankton. Hydrobiologia, 377, 147-159.

Kingsolver, J. G., Diamond, S. E., Siepielski, A. M., and Carlson, S. M. (2012). Synthetic analyses of phenotypic selection in natural populations: lessons, limitations and future directions. Evol. Ecol., 26, 1101-1118.

Kinnison, M.T., Hairston, N.G., Jr \& Hendry, A.P. (2015). Cryptic eco-evolutionary dynamics. Ann. N. Y. Acad. Sci., 1360, 120-144.

Kolar, C.S. \& Lodge, D.M. (2001). Progress in invasion biology: predicting invaders. Trends Ecol. Evol., 16, 199-204.

Kramer, A.M. \& Drake, J.M. (2010). Experimental demonstration of population extinction due to a predator-driven Allee effect. J. Anim. Ecol., 79, 633-639.

Kyle, M., Acharya, K., Weider, L.J., Looper, K. \& Elser, J.J. (2006). Coupling of growth rate and body stoichiometry in Daphnia: a role for maintenance processes? Freshw. Biol., 51, 2087-2095.

Lachish, S., McCallum, H. \& Jones, M. (2009). Demography, disease and the devil: life-history changes in a disease-affected population of Tasmanian devils (Sarcophilus harrisii). J. Anim. Ecol., $78,427-436$.

Lampert, W. \& Trubetskova, I. (1996). Juvenile growth rate as a measure of fitness in Daphnia. Funct. Ecol., 10, 631-635.

Langley, J.M., Shiel, R.J., Nielsen, D.L. \& Green, J.D. (2001). Hatching from the sediment egg-bank, or aerial dispersing? - the use of mesocosms in assessing rotifer biodiversity. In: Rotifera IX. Springer Netherlands, pp. 203-211.

Lässig, M., Mustonen, V. \& Walczak, A.M. (2017). Predicting evolution. Nat. Ecol. Evol., 1, 0077.

Leal, M. C., Best, R. J., Durston, D., El-Sabaawi, R. W., and Matthews, B. (2017a). Stoichiometric traits of stickleback: Effects of genetic background, rearing environment, and ontogeny. Ecol. Evol., 7, 2617-2625.

Leal, M.C., Seehausen, O. \& Matthews, B. (2017b). The ecology and evolution of stoichiometric phenotypes. Trends Ecol. Evol., 32, 108-117.

Lemmen, K.D., Zhou, L., Papakostas, S. \& Declerck, S.A.J. (2020). The Growth Rate Hypothesis as a predictive framework for microevolutionary adaptation to selection for high population growth: an experimental test under phosphorus rich and phosphorus poor conditions. bioRxiv., 150649.

Lenth, R. (2019). emmeans: Estimated Marginal Means, aka Least-Squares Means. https://CRAN.Rproject.org/package $=$ emmeans

LeRoy, C. J., Whitham, T. G., Wooley, S. C., and Marks, J. C. (2007). Within-species variation in foliar chemistry influences leaf-litter decomposition in a Utah river. J. North Am. Benthol., Soc. 26, 426438.

LeRoy, C. J., Wooley, S. C., and Lindroth, R. L. (2012). Genotype and soil nutrient environment influence aspen litter chemistry and in-stream decomposition. Freshw. Sci., 31, 1244-1253.

Leung, C., Breton, S. \& Angers, B. (2016). Facing environmental predictability with different sources of epigenetic variation. Ecol. Evol., 6, 5234-5245. 
Levi, M. P., and Cowling, E. B. (1969). Role of nitrogen in wood deterioration, VII: Physiological adaptation of wood-destroying and other fungi to substrates deficient in nitrogen. Phytopathology, 460-468.

Levins, R. (1968). Evolution in Changing Environments: Some Theoretical Explorations. Princeton University Press.

Liess, A., Guo, J., Lind, M. I., and Rowe, O. (2015). Cool tadpoles from Arctic environments waste fewer nutrients - high gross growth efficiencies lead to low consumer-mediated nutrient recycling in the North. J. Anim. Ecol. 84, 1744-1756.

Liess, A., Rowe, O., Guo, J., Thomsson, G. \& Lind, M.I. (2013). Hot tadpoles from cold environments need more nutrients--life history and stoichiometry reflects latitudinal adaptation. J. Anim. Ecol., 82, 1316-1325.

Lincoln, D.E., Couvet, D. \& Sionit, N. (1986). Response of an insect herbivore to host plants grown in carbon dioxide enriched atmospheres. Oecologia, 69, 556-560.

Lind, P.R. \& Jeyasingh, P.D. (2018). Interactive effects of dietary phosphorus and iron on Daphnia life history : Role of iron in Daphnia reproduction. Limnol. Oceanogr., 63, 1181-1190.

Lino, A., Fonseca, C., Rojas, D., Fischer, E. \& Pereira, M.J.R. (2019). A meta-analysis of the effects of habitat loss and fragmentation on genetic diversity in mammals. Mamm. Biol., 94, 69-76.

Lopes, P.M., Bozelli, R., Bini, L.M., Santangelo, J.M. \& Declerck, S.A.J. (2016). Contributions of airborne dispersal and dormant propagule recruitment to the assembly of rotifer and crustacean zooplankton communities in temporary ponds. Freshw. Biol., 61, 658-669.

Louette, G., Vanoverbeke, J., Ortells, R. \& De Meester, L. (2007). The founding mothers: the genetic structure of newly established Daphnia populations. Oikos, 116, 728-741.

Luijckx, P., Ho, E.K.H., Gasim, M., Chen, S., Stanic, A., Yanchus, C., et al. (2017). Higher rates of sex evolve during adaptation to more complex environments. Proc. Natl. Acad. Sci. U. S. A., 114, 534539.

Lukas, M. \& Wacker, A. (2014). Acclimation to dietary shifts impacts the carbon budgets of Daphnia magna. J. Plankton Res., 36, 848-858.

Lynch, M., and Walsh, B. (1998). Genetics and Analysis of quantitatiVe Traits. Sunderland; Mass, Ml: Sinauer.

MacArthur, R.H. \& Wilson, E.O. (1967). The Theory of Island Biogeography. Princeton University Press, Princeton, NJ.

MacColl, A.D.C. (2011). The ecological causes of evolution. Trends Ecol. Evol., 26, 514-522.

Madritch, M. D., and Hunter, M. D. (2005). Phenotypic variation in oak litter influences short- and long-term nutrient cycling through litter chemistry. Soil Biol. Biochem., 37, 319-327.

Madritch, M., Donaldson, J. R., and Lindroth, R. L. (2006). Genetic identity of Populus tremuloides litter influences decomposition and nutrient release in a mixed forest stand. Ecosystems, 9, 528-537.

Malzahn, A.M., Aberle, N., Clemmesen, C. \& Boersma, M. (2007). Nutrient limitation of primary producers affects planktivorous fish condition. Limnol. Oceanogr., 52, 2062-2071.

Malzahn, A.M., Hantzsche, F., Schoo, K.L., Boersma, M. \& Aberle, N. (2010). Differential effects of nutrient-limited primary production on primary, secondary or tertiary consumers. Oecologia, 162, $35-48$.

Marcus, N.H., Lutz, R., Burnett, W. \& Cable, P. (1994). Age, viability, and vertical distribution of zooplankton resting eggs from an anoxic basin: Evidence of an egg bank. Limnol. Oceanogr., 39, 154-158.

Marin, P., Genitoni, J., Barloy, D., Maury, S., Gibert, P., Ghalambor, C.K., et al. (2020). Biological invasion: The influence of the hidden side of the (epi)genome. Funct. Ecol., 34, 385-400. 
Mather, A., Hancox, D. \& Riginos, C. (2015). Urban development explains reduced genetic diversity in a narrow range endemic freshwater fish. Conserv. Genet., 16, 625-634.

Matthews, B., Aebischer, T., Sullam, K. E., Lundsgaard-Hansen, B., and Seehausen, O. (2016). Experimental evidence of an eco-evolutionary feedback during adaptive divergence. Curr. Biol., 26, 483-489.

Matthews, B., Narwani, A., Hausch, S., Nonaka, E., Peter, H., Yamamichi, M., et al. (2011). Toward an integration of evolutionary biology and ecosystem science. Ecol. Lett., 14, 690-701.

Meirmans, P.G. \& Van Tienderen, P.H. (2004). GENOTYPE and GENODIVE: two programs for the analysis of genetic diversity of asexual organisms. Molecular Ecology Notes, 4, 792-794.

Menezes, B.F., Salces-Ortiz, J., Muller, H., Burlet, N., Martinez, S., Fablet, M., et al. (2018). An attempt to select non-genetic variation in resistance to starvation and reduced chill coma recovery time in Drosophila melanogaster. J. Exp. Biol., 221.

Mergeay, J., Verschuren, D. \& De Meester, L. (2006). Invasion of an asexual American water flea clone throughout Africa and rapid displacement of a native sibling species. Proc. Biol. Sci., 273, 2839-2844.

Meunier, C. L., Boersma, M., El-Sabaawi, R., Halvorson, H. M., Herstoff, E. M., Van de Waal, D. B., et al. (2017). From elements to function: toward unifying ecological stoichiometry and trait-based ecology. Front. Environ. Sci., 5, 18.

Michaloudi, E., Papakostas, S., Stamou, G., Neděla, V., Tihlař́ková, E., Zhang, W., et al. (2018). Reverse taxonomy applied to the Brachionus calyciflorus cryptic species complex: Morphometric analysis confirms species delimitations revealed by molecular phylogenetic analysis and allows the (re)description of four species. PLoS One, 13, e0203168.

Mignon-Grasteau, S., Chantry-Darmon, C., Boscher, M.-Y., Sellier, N., Le Bihan-Duval, E., and Bertin, A. (2017). Genetic determinism of fearfulness, general activity and feeding behavior in chickens and its relationship with digestive efficiency. Behav. Genet., 47, 114-124.

Miousse, I.R., Chalbot, M.-C.G., Lumen, A., Ferguson, A., Kavouras, I.G. \& Koturbash, I. (2015). Response of transposable elements to environmental stressors. Mutat. Res. - Rev. Mut. Res., 765, 19-39.

Moody, E. K., Carson, E. W., Corman, J. R., Espinosa-Pérez, H., Ramos, J., Sabo, J. L., et al. (2018). Consumption explains intraspecific variation in nutrient recycling stoichiometry in a desert fish. Ecology, 99, 1552-1561.

Moody, E.K., Corman, J.R., Elser, J.J. \& Sabo, J.L. (2015). Diet composition affects the rate and N:P ratio of fish excretion. Freshw. Biol., 60, 456-465.

Moody, E.K. \& Wilkinson, G.M. (2019). Functional shifts in lake zooplankton communities with hypereutrophication. Freshw. Biol., 64, 608-616.

Morris, J. L., Puttick, M. N., Clark, J. W., Edwards, D., Kenrick, P., Pressel, S., et al. (2018). The timescale of early land plant evolution. Proc. Natl. Acad. Sci U.S.A., 115, E2274-E2283.

Morrissey, M. B. (2016). Meta-analysis of magnitudes, differences and variation in evolutionary parameters. J. Evol. Biol., 29, 1882-1904.

Mouginot, C., Kawamura, R., Matulich, K.L., Berlemont, R., Allison, S.D., Amend, A.S., et al. (2014). Elemental stoichiometry of Fungi and Bacteria strains from grassland leaf litter. Soil Biol. Biochem., 76, 278-285.

Mouquet, N., Lagadeuc, Y., Devictor, V., Doyen, L., Duputié, A., Eveillard, D., et al. (2015). REVIEW: Predictive ecology in a changing world. J. Appl. Ecol., 52, 1293-1310.

Mueller-Navarra, D. (1995). Evidence that a highly unsaturated fatty acid limits Daphnia growth in nature. Arch. Hydrobiol., 132, 297-297.

Muggeo, V.M.R. (2008). Modeling temperature effects on mortality: multiple segmented relationships with common break points. Biostatistics. 9, 613-620. 
Müller-Navarra, D.C. (1995). Biochemical versus mineral limitation in Daphnia. Limnol. Oceanogr., 40, 1209-1214.

Murdoch, W.W. \& Oaten, A. (1975). Predation and Population Stability. In: Advances in Ecological Research (ed. MacFadyen, A.). Academic Press, pp. 1-131.

Neely, K. G., Myers, J. M., Hard, J. J., and Shearer, K. D. (2008). Comparison of growth, feed intake, and nutrient efficiency in a selected strain of coho salmon (Oncorhynchus kisutch) and its source stock. Aquaculture, 283, 134-140.

Neiman, M., Kay, A. D., and Krist, A. C. (2012). Sensitivity to phosphorus limitation increases with ploidy level in a New Zealand snail. Evolution., 67, 1511-1517.

Ness, R.W., Morgan, A.D., Vasanthakrishnan, R.B., Colegrave, N. \& Keightley, P.D. (2015). Extensive de novo mutation rate variation between individuals and across the genome of Chlamydomonas reinhardtii. Genome Res., 25, 1739-1749.

Noble, D. (2019). metaAidR. GitHub Repository. Available online at: https://github.com/daniel1 noble/metaAidR/

Noble, D. W. A., Lagisz, M., O'dea, R. E., and Nakagawa, S. (2017). Nonindependence and sensitivity analyses in ecological and evolutionary meta-analyses. Mol. Ecol., 26, 2410-2425.

O'Dea, R.E., Noble, D.W.A., Johnson, S.L., Hesselson, D. \& Nakagawa, S. (2016). The role of nongenetic inheritance in evolutionary rescue: epigenetic buffering, heritable bet hedging and epigenetic traps. Environ. Epigenet., 2, dvv014.

Oaten, A. \& Murdoch, W.W. (1975). Functional Response and Stability in Predator-Prey Systems. Am. Nat., 109, 289-298.

Omilian, A.R., Cristescu, M.E.A., Dudycha, J.L. \& Lynch, M. (2006). Ameiotic recombination in asexual lineages of Daphnia. Proc. Natl. Acad. Sci. U. S. A., 103, 18638-18643.

Ott, D., Rall, B.C. \& Brose, U. (2012). Climate change effects on macrofaunal litter decomposition: the interplay of temperature, body masses and stoichiometry. Philos. Trans. R. Soc. Lond. B Biol. Sci., 367, 3025-3032.

Papakostas, S., Michaloudi, E., Proios, K., Brehm, M., Verhage, L., Rota, J., et al. (2016). Integrative taxonomy recognizes evolutionary units despite widespread mitonuclear discordance: Evidence from a rotifer cryptic species complex. Syst. Biol., 65, 508-524.

Pérez, J.E., Nirchio, M., Alfonsi, C. \& Muñoz, C. (2006). The Biology of Invasions: The Genetic Adaptation Paradox. Biol. Invasions, 8, 1115-1121.

Perez, M.F. \& Lehner, B. (2019). Intergenerational and transgenerational epigenetic inheritance in animals. Nat. Cell Biol., 21, 143-151.

Petri, M. (2006). Water Quality of Lake Constance. In: The Rhine (ed. Knepper, T.P.). Springer Berlin Heidelberg, Berlin, Heidelberg, pp. 127-138.

Piepho, M., Martin-Creuzburg, D. \& Wacker, A. (2010). Simultaneous effects of light intensity and phosphorus supply on the sterol content of phytoplankton. PLoS One, 5, e15828.

Pimpinelli, S. \& Piacentini, L. (2020). Environmental change and the evolution of genomes:

Transposable elements as translators of phenotypic plasticity into genotypic variability. Funct. Ecol., 34, 428-441.

Plath, K. \& Boersma, M. (2001). Mineral Limitation of Zooplankton: Stoichiometric Constraints and Optimal Foraging. Ecology, 82, 1260.

Pometti, C. L., Bessega, C. F., Vilardi, J. C., Cialdella, A. M., and Saidman, B. O. (2015). Genetic diversity within and among two Argentinean and one Mexican species of Acacia (Fabaceae): Genetic Diversity in Three Acacia Species. Bot. J. Linn. Soc., 177, 593-606.

Post, D. M., Palkovacs, E. P., Schielke, E. G., and Dodson, S. I. (2008). Intraspecific variation in a predator affects community structure and cascading trophic interactions. Ecology, 89, 2019-2032. 
Prater, C., Wagner, N. D., and Frost, P. C. (2017). Interactive effects of genotype and food quality on consumer growth rate and elemental content. Ecology, 98, 1399-1408.

Prater, C., Wagner, N.D. \& Frost, P.C. (2018). Seasonal effects of food quality and temperature on body stoichiometry, biochemistry, and biomass production in Daphnia populations: Diet and temperature effects on Daphnia. Limnol. Oceanogr., 63, 1727-1740.

Prentis, P.J., Wilson, J.R.U., Dormontt, E.E., Richardson, D.M. \& Lowe, A.J. (2008). Adaptive evolution in invasive species. Trends Plant Sci., 13, 288-294.

Pritchard, D.W., Paterson, R.A., Bovy, H.C. \& Barrios-O'Neill, D. (2017). frair : an R package for fitting and comparing consumer functional responses. Methods Ecol. Evol., 8, 1528-1534.

R Core Team, (2018). R: A Language and Environment for Statistical Computing. \{R Foundation for Statistical Computing\}, Vienna, Austria.

Raffard, A., Santoul, F., Cucherousset, J. \& Blanchet, S. (2019). The community and ecosystem consequences of intraspecific diversity: a meta-analysis: The ecological effects of intraspecific diversity. Biol Rev, 94, 648-661.

Rall, B.C., Brose, U., Hartvig, M., Kalinkat, G., Schwarzmüller, F., Vucic-Pestic, O., et al. (2012). Universal temperature and body-mass scaling of feeding rates. Philos. Trans. R. Soc. Lond. B Biol. Sci., 367, 2923-2934.

Rapp, R.A. \& Wendel, J.F. (2005). Epigenetics and plant evolution: Research review. New Phytol., 168, 81-91.

Ravet, J.L. \& Brett, M.T. (2006). Phytoplankton essential fatty acid and phosphorus content constraints on Daphnia somatic growth and reproduction. Limnol. Oceanogr., 51, 2438-2452.

Real, L.A. (1977). The Kinetics of Functional Response. Am. Nat., 111, 289-300.

Rechavi, O., Houri-Ze'evi, L., Anava, S., Goh, W.S.S., Kerk, S.Y., Hannon, G.J., et al. (2014).

Starvation-induced transgenerational inheritance of small RNAs in C. elegans. Cell, 158, 277-287.

Redfield, A.C. (1958) The Biological Control of Chemical Factors in the Environment. Am. Nat., 46, 205-221.

Reiners, W.A. (1986). Complementary Models for Ecosystems. Am. Nat., 127, 59-73.

Rejmánková, E. (2005). Nutrient resorption in wetland macrophytes: comparison across several regions of different nutrient status. New Phytol., 167, 471-482.

Rey, O., Danchin, E., Mirouze, M., Loot, C. \& Blanchet, S. (2016). Adaptation to Global Change: A Transposable Element-Epigenetics Perspective. Trends Ecol. Evol., 31, 514-526.

Reznick, D.N. \& Ghalambor, C.K. (2001). The population ecology of contemporary adaptations: what empirical studies reveal about the conditions that promote adaptive evolution. Genetica, 112113, 183-198.

Richards, E.J. (2006). Inherited epigenetic variation--revisiting soft inheritance. Nat. Rev. Genet., 7, 395-401.

Rodriguez-Cabal, M. A., Barrios-Garcia, M. N., Rudman, S. M., McKown, A. D., Sato, T., and Crutsinger, G. M. (2017). It is about time: genetic variation in the timing of leaf-litter inputs influences aquatic ecosystems. Freshw. Biol. 62, 356-365.

Rogers, D. (1972). Random Search and Insect Population Models. J. Anim. Ecol., 41, 369-383.

Rollins, L.A., Moles, A.T., Lam, S., Buitenwerf, R., Buswell, J.M., Brandenburger, C.R., et al. (2013). High genetic diversity is not essential for successful introduction. Ecol. Evol., 3, 4501-4517.

Rollins, L.A., Richardson, M.F. \& Shine, R. (2015). A genetic perspective on rapid evolution in cane toads (Rhinella marina). Mol. Ecol., 24, 2264-2276.

Roman, J. \& Darling, J.A. (2007). Paradox lost: genetic diversity and the success of aquatic invasions. Trends Ecol. Evol., 22, 454-464. 
Rosenbaum, B. \& Rall, B.C. (2018). Fitting functional responses: Direct parameter estimation by simulating differential equations. Methods Ecol. Evol., 9, 2076-2090.

Rothhaupt, K.O. (1990). Changes of the functional responses of the rotifers Brachionus rubens and Brachionus calyciflorus with particle sizes. Limnol. Oceanogr., 35, 24-32.

Rothhaupt, K.O. (1995). Algal nutrient limitation affects rotifer growth rate but not ingestion rate. Limnol. Oceanogr., 40, 1201-1208.

Roy Chowdhury, P., Frisch, D., Becker, D., Lopez, J.A., Weider, L.J., Colbourne, J.K., et al. (2015). Differential transcriptomic responses of ancient and modern Daphnia genotypes to phosphorus supply. Mol. Ecol., 24, 123-135.

Rudman, S. M., Barbour, M. A., Csilléry, K., Gienapp, P., Guillaume, F., Hairston, N. G., et al. (2018). What genomic data can reveal about eco-evolutionary dynamics. Nat. Ecol. Evol., 2, 9-15.

Rudman, S.M., Goos, J.M., Burant, J.B., Brix, K.V., Gibbons, T.C., Brauner, C.J., et al. (2019). lonome and elemental transport kinetics shaped by parallel evolution in threespine stickleback. Ecol. Lett. $22,645-653$.

Rudman, S. M., and Schluter, D. (2016). Ecological impacts of reverse speciation in threespine stickleback. Curr. Biol., 26, 490-495.

Sanders, A.J. \& Taylor, B.W. (2018). Using ecological stoichiometry to understand and predict infectious diseases. Oikos, 127, 1399-1409.

Sarnelle, O., White, J.D., Geelhoed, T.E., Kozel, C.L. \& Smith, R. (2015). Type III functional response in the zebra mussel, Dreissena polymorpha. Can. J. Fish. Aquat. Sci., 72, 1202-1207.

Sarnelle, O. \& Wilson, A.E. (2008). Type III functional response in Daphnia. Ecology, 89, 1723-1732.

Schade, J. D., Kyle, M., Hobbie, S. E., Fagan, W. F., and Elser, J. J. (2003). Stoichiometric tracking of soil nutrients by a desert insect herbivore. Ecol. Lett. 6, 96-101.

Scheuerl, T. \& Stelzer, C.-P. (2013). Patterns and dynamics of rapid local adaptation and sex in varying habitat types in rotifers. Ecol. Evol., 3, 4253-4264.

Schlaepfer, D.R., Braschler, B., Rusterholz, H.-P. \& Baur, B. (2018). Genetic effects of anthropogenic habitat fragmentation on remnant animal and plant populations: a meta-analysis. Ecosphere, 9.

Schluter, D. (2000). The Ecology of Adaptive Radiation. Oxford: Oxford University Press.

Schlüter, L., Lohbeck, K.T., Gutowska, M.A., Gröger, J.P., Riebesell, U. \& Reusch, T.B.H. (2014).

Adaptation of a globally important coccolithophore to ocean warming and acidification. Nat. Clim. Chang., 4, 1024-1030.

Schrey, A.W., Coon, C.A.C., Grispo, M.T., Awad, M., Imboma, T., McCoy, E.D., et al. (2012).

Epigenetic Variation May Compensate for Decreased Genetic Variation with Introductions: A Case Study Using House Sparrows (Passer domesticus) on Two Continents. Genet. Res. Int., 2012, 979751.

Schoener, T.W. (2011). The newest synthesis: understanding the interplay of evolutionary and ecological dynamics. Science, 331, 426-429.

Schoo, K.L., Aberle, N., Malzahn, A.M. \& Boersma, M. (2012). Food quality affects secondary consumers even at low quantities: an experimental test with larval European lobster. PLoS One, 7, e33550.

Schröder, T. (2005). Diapause in Monogonont Rotifers. Hydrobiologia, 546, 291-306.

Schulz, K.L. \& Sterner, R.W. (1999). Phytoplankton phosphorus limitation and food quality for Bosmina. Limnol. Oceanogr., 44, 1549-1556.

Schweiger, O., Biesmeijer, J.C., Bommarco, R., Hickler, T., Hulme, P.E., Klotz, S., et al. (2010). Multiple stressors on biotic interactions: how climate change and alien species interact to affect pollination. Biol. Rev. Camb. Philos. Soc., 85, 777-795. 
Schweitzer, J., Bailey, J., Rehill, B., Martinsen, G., Hart, S., Lindroth, R., et al. (2004). Genetically based trait in a dominant tree affects ecosystem processes. Ecol. Lett., 7, 127-134.

Seidendorf, B., Meier, N., Petrusek, A., Boersma, M., Streit, B. \& Schwenk, K. (2010). Sensitivity of Daphnia species to phosphorus-deficient diets. Oecologia, 162, 349-357.

Seifert, L.I., de Castro, F., Marquart, A., Gaedke, U., Weithoff, G. \& Vos, M. (2014). Heated relations: temperature-mediated shifts in consumption across trophic levels. PLoS One, 9, e95046.

Sentis, A., Bertram, R., Dardenne, N., Ramon-Portugal, F., Espinasse, G., Louit, I., et al. (2018). Evolution without standing genetic variation: change in transgenerational plastic response under persistent predation pressure. Heredity, 121, 266-281.

Serra, M., García-Roger, E. M., Ortells, R. \& Carmona, M. J. (2019). Cyclically parthenogenetic rotifers and the theories of population and evolutionary ecology. Limnetica, 38, 67-93.

Serra, M. \& Snell, T.W. (2009). Sex Loss in Monogonont Rotifers. In: Lost Sex: The Evolutionary Biology of Parthenogenesis (eds. Schön, I., Martens, K. \& Dijk, P.). Springer Netherlands, Dordrecht, pp. 281-294.

Seudre, O., Vanhoenacker, E., Mauger, S., Coudret, J. \& Roze, D. (2020). Genetic variability and transgenerational regulation of investment in sex in the monogonont rotifer Brachionus plicatilis. J. Evol. Biol., 33, 112-120.

Sheldon, E.L., Schrey, A., Andrew, S.C., Ragsdale, A. \& Griffith, S.C. (2018). Epigenetic and genetic variation among three separate introductions of the house sparrow (Passer domesticus) into Australia. R Soc Open Sci, 5, 172185.

Sherman, R.E., Chowdhury, P.R., Baker, K.D., Weider, L.J. \& Jeyasingh, P.D. (2017). Genotypespecific relationships among phosphorus use, growth and abundance in Daphnia pulicaria. R. Soc. Open Sci., 4, 170770.

Shi, W., Chen, X., Gao, L., Xu, C.-Y., Ou, X., Bossdorf, O., et al. (2018). Transient Stability of Epigenetic Population Differentiation in a Clonal Invader. Front. Plant Sci., 9, 1851.

Shtonda, B.B. \& Avery, L. (2006). Dietary choice behavior in Caenorhabditis elegans. J. Exp. Biol., 209, 89-102.

Silfver, T., Mikola, J., Rousi, M., Roininen, H., and Oksanen, E. (2007). Leaf litter decomposition differs among genotypes in a local Betula pendula population. Oecologia, 152, 707-714.

Simpson, S.J. \& Raubenheimer, D. (2012). Mechanisms of Nutritional Regulation. In: The Nature of Nutrition, A Unifying Framework from Animal Adaptation to Human Obesity. Princeton University Press, pp. 35-56.

Smil, V. (2000). PHOSPHORUS IN THE ENVIRONMENT: Natural Flows and Human Interferences. Annu. Rev. Energy Environ., 25, 53-88.

Smith, H.A. \& Snell, T.W. (2012). Rapid evolution of sex frequency and dormancy as hydroperiod adaptations. J. Evol. Biol., 25, 2501-2510.

Smith, H.A. \& Snell, T.W. (2014). Differential evolution of asexual and sexual females in a benign culture environment. Int. Rev. Hydrobiol., 99, 117-124.

Smith, V.H. \& Schindler, D.W. (2009). Eutrophication science: where do we go from here? Trends Ecol. Evol., 24, 201-207.

Smith, V.H., Tilman, G.D. \& Nekola, J.C. (1999). Eutrophication: impacts of excess nutrient inputs on freshwater, marine, and terrestrial ecosystems. Environ. Pollut., 100, 179-196.

Snell, T.W. (2014). Rotifers as models for the biology of aging. Int. Rev. Hydrobiol., 99, 84-95.

Snell, T.W., Johnston, R.K., Matthews, A.B., Zhou, H., Gao, M. \& Skolnick, J. (2018). Repurposed FDA-approved drugs targeting genes influencing aging can extend lifespan and healthspan in rotifers. Biogerontology, 19, 145-157. 
Snell-Rood, E.C., Swanson, E.M., Espeset, A., Jaumann, S., Philips, K., Walker, C., et al. (2020). Nutritional constraints on brain evolution: Sodium and nitrogen limit brain size. Evolution, 74, $2304-$ 2319 .

So, M., Ohtsuki, H., Makino, W., Ishida, S., Kumagai, H., Yamaki, K.G., et al. (2015). Invasion and molecular evolution of Daphnia pulex in Japan. Limnol. Oceanogr., 60, 1129-1138.

Sommer, U., Adrian, R., De Senerpont Domis, L., Elser, J.J., Gaedke, U., Ibelings, B., et al. (2012). Beyond the Plankton Ecology Group (PEG) Model: Mechanisms Driving Plankton Succession. Annu. Rev. Ecol. Evol. Syst., 43, 429-448.

Song, B.-M., Faumont, S., Lockery, S. \& Avery, L. (2013). Recognition of familiar food activates feeding via an endocrine serotonin signal in Caenorhabditis elegans. Elife, 2, e00329.

Stanley-Samuelson, D.W. (1994). The Biological Significance of Prostaglandins and Related Eicosanoids in Invertebrates. Am. Zool., 34, 589-598.

Stapley, J., Santure, A.W. \& Dennis, S.R. (2015). Transposable elements as agents of rapid adaptation may explain the genetic paradox of invasive species. Mol. Ecol., 24, 2241-2252.

Stelzer, C.-P. (2011). The cost of sex and competition between cyclical and obligate parthenogenetic rotifers. Am. Nat., 177, E43-53.

Stelzer, C.-P. (2017). Extremely short diapause in rotifers and its fitness consequences. Hydrobiologia, 796, 255-264.

Stelzer, C.-P. \& Snell, T.W. (2003). Induction of sexual reproduction in Brachionus plicatilis (Monogononta, Rotifera) by a density-dependent chemical cue. Limnol. Oceanogr., 48, 939-943.

Sterner, R.W. (1990). The Ratio of Nitrogen to Phosphorus Resupplied by Herbivores: Zooplankton and the Algal Competitive Arena. Am. Nat., 136, 209-229.

Sterner, R.W. \& Elser, J. (2002). Ecological Stoichiometry. Princeton University Press, Princeton, N.J.

Sterner, R.W., Elser, J.J. \& Hessen, D.O. (1992). Stoichiometric relationships among producers, consumers and nutrient cycling in pelagic ecosystems. Biogeochemistry, 17, 49-67.

Sterner, R.W. \& Hessen, D.O. (1994). Algal nutrient limitation and the nutrition of aquatic herbivores. Annu. Rev. Ecol. Syst., 25, 1-29.

Sterner, R. W., Hood, J. M., Kearney, M. R., Urabe, J., and Raubenheimer, D. (2015). Couples that have chemistry: when ecological theories meet. Oikos, 124, 917-919.

Sterner, R.W. \& Robinson, J.L. (1994) Thresholds for growth in Daphnia magna with high and low phosphorus diets. Limnol. Oceanogr., 39, 1228-1232.

Sterner, R.W. \& Smith, R.F. (1993). CLEARANCE, INGESTION AND RELEASE OF N AND P BY DAPHNIA-OBTUSA FEEDING ON SCENEDESMUS-ACUTUS OF VARYING QUALITY. Bull. Mar. Sci., $53,228-239$.

Stocker, T.F., Qin, D., Plattner, G.-K., Tignor, M.M.B., Allen, S.K., Boschung, J., et al. (2014). Climate Change 2013: The Physical Science Basis. Contribution of Working Group I to the Fifth Assessment Report of IPCC the Intergovernmental Panel on Climate Change. Cambridge University Press, Cambridge.

Stockner, J.G., Rydin, E. \& Hyenstrand, P. (2000). Cultural oligotrophication: causes and consequences for fisheries resources. Fisheries, 25, 7-14.

Suzuki-Ohno, Y., Kawata, M. \& Urabe, J. (2012). Optimal feeding under stoichiometric constraints: a model of compensatory feeding with functional response. Oikos, 121, 569-578.

Swanson, E.M., Espeset, A., Mikati, I., Bolduc, I., Kulhanek, R., White, W.A., et al. (2016). Nutrition shapes life-history evolution across species. Proc. Biol. Sci., 283.

Tarazona, E., García-Roger, E.M. \& Carmona, M.J. (2017). Experimental evolution of bet hedging in rotifer diapause traits as a response to environmental unpredictability. Oikos, 126, 1162-1172. 
Taylor, D.L. \& Collie, J.S. (2003). Effect of temperature on the functional response and foraging behavior of the sand shrimp Crangon septemspinosa preying on juvenile winter flounder Pseudopleuronectes americanus. Mar. Ecol. Prog. Ser., 263, 217-234.

Tillberg, J.-E., Barnard, T. \& Rowley, J.R. (1984). PHOSPHORUS STATUS AND CYTOPLASMIC STRUCTURES IN SCENEDESMUS (CHLOROPHYCEAE) UNDER DIFFERENT METABOLIC REGIMES1. J. Phycol., 20, 124-136.

Tobler, M., Alba, D.M., Arias-Rodríguez, L. \& Jeyasingh, P.D. (2016). Using replicated evolution in extremophile fish to understand diversification in elemental composition and nutrient excretion. Freshw. Biol., 61, 158-171.

Townsend, A. R., Cleveland, C. C., Asner, G. P., and Bustamante, M. M. C. (2007). Controls over foliar N:P ratios in tropical rain forests. Ecology, 88, 107-118.

Trakimas, G., Krams, R., Krama, T., Kortet, R., Haque, S., Luoto, S., et al. (2019). Ecological stoichiometry: a link between developmental speed and physiological stress in an omnivorous insect. Front. Behav. Neurosci., 13, 42.

Trussell, G.C. \& Schmitz, O.J. (2012). Species functional traits, trophic control and the ecosystem consequences of adaptive foraging in the middle of food chains. In: Trait-Mediated Indirect Interactions: Ecological and Evolutionary Perspectives. Cambridge University Press, pp. 324-338.

Tsai, C.-H., Miki, T., Chang, C.-W., Ishikawa, K., Ichise, S., Kumagai, M., et al. (2014). Phytoplankton functional group dynamics explain species abundance distribution in a directionally changing environment.

Tummers, B. (2006). DataThief III.

Turcotte, M. M., Reznick, D. N., and Hare, J. D. (2011). The impact of rapid evolution on population dynamics in the wild: experimental test of eco-evolutionary dynamics. Ecol. Lett., 14, 1084-1092.

Turner, C.B., Wade, B.D., Meyer, J.R., Sommerfeld, B.A. \& Lenski, R.E. (2017). Evolution of organismal stoichiometry in a long-term experiment with Escherichia coli. $R$ Soc Open Sci, 4, 170497.

Urabe, J., Shimizu, Y. \& Yamaguchi, T. (2018). Understanding the stoichiometric limitation of herbivore growth: the importance of feeding and assimilation flexibilities. Ecol. Lett., 21, 197-206.

Urabe, J., Togari, J. \& Elser, J.J. (2003). Stoichiometric impacts of increased carbon dioxide on a planktonic herbivore. Glob. Chang. Biol., 9, 818-825.

Uszko, W., Diehl, S., Pitsch, N., Lengfellner, K. \& Müller, T. (2015). When is a type III functional response stabilizing? Theory and practice of predicting plankton dynamics under enrichment. Ecology, 96, 3243-3256.

Uszko, W., Diehl, S. \& Wickman, J. (2020). Fitting functional response surfaces to data: a best practice guide. Ecosphere, 11, 463.

van de Waal, D.B., Verschoor, A.M., Verspagen, J.M.H., van Donk, E. \& Huisman, J. (2010). Climatedriven changes in the ecological stoichiometry of aquatic ecosystems. Front. Ecol. Environ., 8, 145152.

Van Donk, E., Lürling, M., Hessen, D.O. \& Lokhorst, G.M. (1997). Altered cell wall morphology in nutrient-deficient phytoplankton and its impact on grazers. Limnol. Oceanogr., 42, 357-364.

Vanni, M.J. (2002). Nutrient Cycling by Animals in Freshwater Ecosystems. Annu. Rev. Ecol. Syst., 33, 341-370.

Vanschoenwinkel, B., Seaman, M. \& Brendonck, L. (2010). Hatching phenology, life history and egg bank size of fairy shrimp Branchipodopsis spp. (Branchiopoda, Crustacea) in relation to the ephemerality of their rock pool habitat. Aquat Ecol., 44, 771-780.

Verbeek, L., Gall, A., Hillebrand, H. \& Striebel, M. (2018). Warming and oligotrophication cause shifts in freshwater phytoplankton communities. Glob. Chang. Biol., 24, 4532-4543. 
Verhoeven, K.J.F. \& Preite, V. (2014). Epigenetic variation in asexually reproducing organisms. Evolution, 68, 644-655.

Verschoor, A.M., Van Dijk, M.A., Huisman, J. \& Van Donk, E. (2013). Elevated CO2 concentrations affect the elemental stoichiometry and species composition of an experimental phytoplankton community. Freshw. Biol., 58, 597-611.

Verschoor, A.M., Zadereev, Y.S. \& Mooij, W.M. (2007). Infochemical-mediated trophic interactions between the rotifer Brachionus calyciflorus and its food algae. Limnol. Oceanogr., 52, 2109-2119.

Verspagen, J.M.H., Van de Waal, D.B., Finke, J.F., Visser, P.M. \& Huisman, J. (2014). Contrasting effects of rising $\mathrm{CO} 2$ on primary production and ecological stoichiometry at different nutrient levels. Ecol. Lett., 17, 951-960.

Violle, C., Enquist, B.J., McGill, B.J., Jiang, L., Albert, C.H., Hulshof, C., et al. (2012). The return of the variance: intraspecific variability in community ecology. Trends Ecol. Evol., 27, 244-252.

Vogt, G. (2017). Facilitation of environmental adaptation and evolution by epigenetic phenotype variation: insights from clonal, invasive, polyploid, and domesticated animals. Environmental Epigenetics.

Visanuvimol, L., and Bertram, S. M. (2011). How dietary phosphorus availability during development influences condition and life history traits of the cricket, Acheta domesticas. J. Insect Sci., 11, 1-17.

Vrede, T., Andersen, T. \& Hessen, D.O. (1999). Phosphorus distribution in three crustacean zooplankton species. Limnol. Oceanogr., 44, 225-229.

Wadgymar, S.M., Lowry, D.B., Gould, B.A., Byron, C.N., Mactavish, R.M. \& Anderson, J.T. (2017). Identifying targets and agents of selection: innovative methods to evaluate the processes that contribute to local adaptation. Methods Ecol. Evol., 8, 738-749.

Wagner, D. O., and Aspenberg, P. (2011). Where did bone come from?: An overview of its evolution. Acta Orthopaed., 82, 393-398.

Walczyńska, A., Franch-Gras, L. \& Serra, M. (2017). Empirical evidence for fast temperaturedependent body size evolution in rotifers. Hydrobiologia, 796, 191-200.

Wallace, R.L. \& Snell, T.W. (2010). 8 - PHYLUM ROTIFERA. In: Ecology and Classification of North American Freshwater Invertebrates (Second Edition) (eds. Thorp, J.H. \& Covich, A.P.). Academic Press, San Diego, pp. 195-254.

Walsh, M.R., Castoe, T., Holmes, J., Packer, M., Biles, K., Walsh, M., et al. (2016). Local adaptation in transgenerational responses to predators. Proc. Biol. Sci., 283.

Walsh, M.R., Cooley, F., 4th, Biles, K. \& Munch, S.B. (2015). Predator-induced phenotypic plasticity within- and across-generations: a challenge for theory? Proc. Biol. Sci., 282, 20142205.

Walsh, M.R. \& Post, D.M. (2011). Interpopulation variation in a fish predator drives evolutionary divergence in prey in lakes. Proc. Biol. Sci., 278, 2628-2637.

Walsh, M.R. \& Reznick, D.N. (2008). Interactions between the direct and indirect effects of predators determine life history evolution in a killifish. Proc. Natl. Acad. Sci. U. S. A., 105, 594-599.

Weaver, I. C. G., Cervoni, N., Champagne, F. A., D'Alessio, A. C., Sharma, S., Seckl, J. R., et al. (2004). Epigenetic programming by maternal behavior. Nat. Neurosci., 7, 847-854.

Weider, L.J., Glenn, K.L., Kyle, M. \& Elser, J.J. (2004). Associations among ribosomal (r)DNA intergenic spacer length, growth rate, and C:N:P stoichiometry in the genus Daphnia. Limnol. Oceanogr., 49, 1417-1423.

Whitham, T., Bailey, J., Schweitzer, J., Shuster, S., Bangert, R., Leroy, C., et al. (2006). A framework for community and ecosystem genetics: from genes to ecosystems. Nat. Rev. Genet. 7, 510-523.

Wilhoit, L.R. \& Mittler, T.E. (1991). Biotypes and Clonal Variation in Greenbug (Homoptera: Aphididae) Populations from a Locality in California. Environ. Entomol., 20, 757-767. 
Williams, R.J.P. \& Fraústo da Silva, J.J.R. (1996). The Natural Selection of the Chemical Elements. Clarendon, Oxford.

Wogan, G.O.U., Yuan, M.L., Mahler, D.L. \& Wang, I.J. (2020). Genome-wide epigenetic isolation by environment in a widespread Anolis lizard. Mol. Ecol., 29, 40-55.

Wood, Z.T., Palkovacs, E.P. \& Kinnison, M.T. (2018). Eco-evolutionary Feedbacks from Non-target Species Influence Harvest Yield and Sustainability. Sci. Rep., 8, 6389.

Xi, Y.-L., Huang, K.-Q., Pan, L., Zhu, H., Ge, Y.-L., Wen, X.-L., et al. (2019). Rapid adaptation of Brachionus angularis (Rotifera) to invasion by Brachionus calyciflorus. Hydrobiologia, 844, 31-42.

Yamamichi, M., Meunier, C.L., Peace, A., Prater, C. \& Rúa, M.A. (2015). Rapid evolution of a consumer stoichiometric trait destabilizes consumer--producer dynamics. Oikos, 124, 960-969.

Zechmeister-Boltenstern, S., Keiblinger, K.M., Mooshammer, M., Peñuelas, J., Richter, A., Sardans, J., et al. (2015). The application of ecological stoichiometry to plant-microbial-soil organic matter transformations. Ecol. Monogr., 85, 133-155.

Zenni, R.D. \& Nuñez, M.A. (2013). The elephant in the room: the role of failed invasions in understanding invasion biology. Oikos, 122, 801-815.

Zhou, L., \& Declerck, S.A.J. (2019). Herbivore consumers face different challenges along opposite sides of the stoichiometric knife-edge. Ecol. Lett., 22, 2018-2027.

Zhou, L., \& Declerck, S.A.J. (2020). Maternal effects in zooplankton consumers are not only mediated by direct but also by indirect effects of phosphorus limitation. Oikos, 129, 766-774.

Zhou, L., Lemmen, K.D., Zhang, W. \& Declerck, S.A.J. (2018). Direct and Indirect Effects of Resource P-Limitation Differentially Impact Population Growth, Life History and Body Elemental Composition of a Zooplankton Consumer. Front. Microbiol., 9, 172. 




\section{Chapter 8}

Thesis summary \& About the author 



\section{Summary}

Every organism has a unique set of elemental requirements, and face the fundamental challenge of obtaining these elements for growth and reproduction. Due to differences in uptake and storage of elements, the composition of autotrophs often does not meet the requirements of their consumers, resulting in a stoichiometric mismatch. Such mismatches have a negative effect on the fitness of primary consumers however, the detrimental impact can potentially be mitigated. Phenotypic plasticity, which is a non-heritable response to an environmental trigger that occurs within a generation, may allow consumers to modify traits such as behaviour or metabolism in response to elemental limitation. Alternatively, selection may act upon heritable phenotypic variation, favouring traits that diminish the effect of mismatch and shifting the mean trait value of the population over the course of several generations. As both the magnitude and the frequency of stoichiometric mismatches are predicted to be exacerbated by anthropogenic changes to biogeochemical cycles, it is becoming increasingly important for us to understand how consumers respond to elemental limitation.

Ecological stoichiometry is a scientific framework that simplifies biological interactions into their elemental building blocks so that they can be viewed in a manner that is analogous to a chemical reaction. This framework focuses on the relative abundance and flow of elements within ecosystems to understand patterns of biodiversity and ecological processes. Ecological stoichiometry also has the potential to advance our understanding of evolutionary trajectories, by identifying ecologically relevant elemental mismatches that may act as selective agents. However, only a minority of studies utilizing the ecological stoichiometry framework have addressed evolutionary questions so far. Thus, the aim of this thesis was to further incorporate an evolutionary perspective into the ecological stoichiometric framework to understand how stoichiometric mismatches shape the phenotypes of primary consumers on ecologically relevant timescales.

Intraspecific variation is generally considered essential for rapid microevolutionary change. Although it has been acknowledged that stoichiometric traits (i.e., elemental composition, acquisition, assimilation, allocation, and excretion) are not fixed within a species, there has yet to be a comprehensive synthesis of the degree to which these traits vary and how this variation differs among traits. Using a metaanalysis of common garden studies, we documented substantial intraspecific genetic variation in stoichiometric traits (Chapter 2). The magnitude of variation in elemental composition was less than life history traits that were measured in the same studies. However, the extent of variation was greater for acquisition, assimilation, allocation, and excretion. This study suggests that there is significant 
potential for stoichiometric traits, especially those related to regulating elemental content, to rapidly evolve and impact ecosystem processes.

As we established in Chapter 2 that stoichiometric traits have the potential to evolve, we next investigated whether microevolutionary responses to selection could be predicted by the ecological stoichiometric framework. The growth rate hypothesis states that body $P$ content of invertebrate organisms will be positively related to its somatic growth rate because somatic growth requires ribosomal RNA which is a P rich molecule. In Chapter 3, we tested if this hypothesis could predict the elemental composition of consumer populations, and if its' predictive capacity was dependent on the extent of consumer-resource stoichiometric mismatch. Using experimental evolution, I selected for fast population growth rates in replicated, genetically diverse populations provided either high or low phosphorus resources. Populations selected with both high and low phosphorus resources had significantly higher population growth rates than the ancestral populations. However, the anticipated positive relationship between body Pcontent and growth rate was only observed in the high phosphorus selected populations. Our results demonstrate that while the growth rate hypothesis can act as a predictive framework, its success is dependent on the environmental context under which selection takes place, as well as the genetic background of the genotypes under consideration.

Selection on standing genetic variation, as seen in Chapter 3, is widely regarded as the primary process by which rapid adaptation occurs. However, some populations with low levels of genetic diversity are nevertheless successful in adverse environmental conditions. The production of de novo heritable variation has been proposed as an alternative pathway for adaptation to occur however, empirical evidence is currently lacking for its relevance on ecological timescales. Therefore, we wanted to explore whether heritable adaptation could rapidly occur in response to stoichiometric mismatch in consumer populations that initially lack genetic diversity (Chapter 4). To investigate this question, I exposed replicate monoclonal populations with either high or low phosphorus resources to a culturing regime that favoured phenotypic variants with fast population growth rates. After six generations we observed heritable adaptation to nutrient limitation in the populations with a low phosphorus exposure history. However, the capacity for such a response was only observed in one of the two clones tested. This study provides one of the first clear examples of a rapid heritable adaptive response resulting from exposure to a stressful environment in a population lacking standing genetic variation. These results suggest that de novo sources of phenotypic variation may play an important role in facilitating adaptation in populations with low genetic diversity. 
In Chapter $\mathbf{3}$ and Chapter 4, we established the capacity of heritable changes to mitigate the impact of stoichiometric mismatch between primary consumers and their resources over several generations. As resource elemental composition may vary within a consumer's life span, plastic responses may play an important role in responding to stoichiometric mismatch. Consumers may display plastic modifications to their ingestion rate in response to elemental content at high resource densities. However, the effects of stoichiometric mismatch have not been quantified across an ecologically relevant range of resource densities (i.e., the consumer functional response). To investigate the effect of stoichiometric mismatch on a primary consumer's functional response, I preformed a series of ingestion experiments with resources of varying elemental and biochemical content (Chapter 5). We demonstrated that at high but not low food densities, consumers with low phosphorus resources exhibit elevated ingestion rates compared to consumer with high phosphorus resources. In systems with high resource density, consumers-resource stoichiometric mismatches lead to increased consumer ingestion to compensate for a deficiency of $P$ in the resource. Plastic responses of consumers in response to elemental content therefore require further investigation as they may impact trophic interactions.

In Chapter 6, I connect the findings of the previous chapters and present the four main takeaway messages of this thesis. First, substantial intraspecific variation exists in stoichiometric traits. Second, stoichiometric mismatches shape microevolutionary trajectories. Third, cryptic evolution occurs in response to phosphorus limitation. Fourth, the ecological stoichiometric framework can be used to anticipate phenotypic responses, but it requires further development to be a more general predictive framework. This thesis demonstrates that primary consumers have the capacity to mitigate the negative effects of stoichiometric mismatches using both heritable and non-heritable mechanisms. By incorporating biological realism (e.g. intraspecific variation, alternative adaptive pathways) into the ecological stoichiometric framework, we can improve our capacity to predict organismal responses to changes in elemental availability. 


\section{Samenvatting}

leder organisme heeft een fundamentele behoefte aan elementen en wordt geconfronteerd met de uitdaging om deze elementen te verwerven voor de groei en voortplanting. Als gevolg van verschillen in de manier waarop autotrofe organismen elementen opnemen en opslaan wijkt de samenstelling van hun elementen soms sterk af van de behoeften van hun primaire consumenten. Zo'n stoichiometrische discrepantie heeft doorgaans negatieve gevolgen voor de fitness van consumenten alhoewel consumenten ook manieren hebben om hiermee om te gaan. Fenotypische plasticiteit laat een organisme toe om binnen de termijn van een generatie op een stoichiometrisch discrepantie te reageren via veranderingen in gedrag of metabolisme. Anderzijds kan selectie op overerfbare fenotypische variatie over de tijdspanne van enkele generaties leiden tot genetische veranderingen in de populaties met als gevolg een verhoogd vermogen om met de gevolgen van stoichiometrische discrepantie om te gaan. Aangezien anthropogene veranderingen van biogeochemische cycli worden verondersteld in toenemende mate te zullen leiden tot veranderingen in de relatieve verhoudingen van elementen in het milieu, is het van belang een beter inzicht te verwerven in de manier waarop populaties van consumenten reageren op stoichiometrische discrepanties.

Ecologische stoichiometrie is een wetenschappelijk raamwerk waarin ecologische interacties worden beschouwd op het niveau van elementen. Dit raamwerk richt zich voornamelijk op de verhoudingen en fluxen van elementen binnen ecosystemen met het doel een beter inzicht te verwerven in ecologische processen en patronen van biodiversiteit. Ecologische stoichiometrie kan ons ook inzichten verschaffen in het verloop van evolutionaire veranderingen, bijvoorbeeld in respons op selectiedruk door stoichiometrische discrepanties. Ondanks dit potentieel bestaat er tot op heden slechts een beperkt aantal studies die ecologische stoichiometrie aanwenden voor het beantwoorden van ecoevolutionaire vraagstukken. Het doel van deze thesis was derhalve een verdere integratie van een evolutionair perspectief in de ecologische stoichiometrie te bevorderen. Speciale aandacht ging hierbij uit naar de vraag hoe stoichiometrische discrepanties de evolutie van het fenotype van primaire consumenten kunnen beïnvloeden op een ecologisch relevante tijdschaal.

Intraspecifieke genetische variatie is een voorwaarde voor micro-evolutionaire veranderingen. Hoewel algemeen erkend wordt dat stoichiometrisch relevante kenmerken (meer bepaald elementsamenstelling, acquisitie, assimilatie, allocatie, en excretie) niet onveranderlijk zijn binnen soorten, is er tot op heden nog geen overzicht over de mate waarin deze kenmerken variëren en hoe deze variatie 
verschilt tussen kenmerken. Via een meta-analyse van relevante 'common garden' studies (Hoofdstuk 2) hebben we het bestaan van substantiële intraspecifieke genetische variatie in stoichiometrische kenmerken aangetoond (Hoofdstuk 2). Hoewel de grootte van de variatie in elementsamenstelling van organismen kleiner bleek dan die van levensgeschiedeniskenmerken was die groter voor acquisitie, assimilatie, allocatie, en excretie. Deze studie suggereert dus dat stoichiometrische kenmerken mogelijks aan snelle evolutionaire veranderingen onderhevig kunnen zijn, vooral die kenmerken die betrekking hebben op de regulatie van de elementsamenstelling van organismen. Dit zijn ook kenmerken met een potentieel grote invloed op ecosysteemprocessen.

In Hoofdstuk 3 onderzochten we vervolgens in hoeverre micro-evolutionaire veranderingen kunnen voorspeld worden met behulp van ecologische stoichiometrie. De 'groeisnelheidshypothese', een belangrijke hypothese in de ecologische stoichiometrie, veronderstelt een causaal verband tussen de concentratie aan fosfor in het lichaam van een ongewerveld organisme en de capaciteit tot snelle somatische lichaamsgroei doordat lichaamsgroei fosforrijk ribosomaal RNA vereist. Voor dit hoofdstuk hebben we getest of deze hypothese in staat is evolutionaire veranderingen in de elementsamenstelling van populaties van een primaire consument te voorspellen, en hebben we onderzocht in hoeverre deze voorspelbaarheid afhankelijk is van de graad van stoichiometrische discrepantie met de voedselbron. Gebruik makend van experimentele evolutie, selecteerden we voor snelle populatiegroei in gerepliceerde, genetisch diverse populaties die voorzien werden enerzijds met fosforrijk voedsel, en anderzijds met fosfor-arm voedsel. In beide behandelingen evolueerden populaties naar hogere populatiegroeisnelheden vergeleken met voorouderlijke populaties. Enkel voor populaties in de behandeling met fosforrijk voedsel werd, in overeenstemming met de hypothese, een toename van het fosforgehalte in het lichaam van de consumenten gevonden. Onze resultaten tonen dus aan dat de mate waarin de groeisnelheidshypothese evolutie kan voorspellen afhangt van de omgevingscontext waaronder selectie plaatsvindt.

Selectie op bestaande genetische variatie wordt algemeen beschouwd als het belangrijkste proces dat snelle evolutionaire aanpassing mogelijk maakt. Niettemin zijn er populaties bekend die ondanks een heel lage genetische diversiteit onder veranderlijke en moeilijke omstandigheden persisteren. Anderzijds wint de gedachte veld dat snelle adaptatie ook tot stand kan komen door het ontstaan van nieuwe overerfbare variatie. Het is evenwel nog niet duidelijk hoe belangrijk zo'n mechanismen zijn voor adaptatie op een ecologisch relevante tijdschaal. Doel van Hoofdstuk 4 was na te gaan of overerfbare adaptatie tot stand kan komen in populaties van primaire consumenten in 
afwezigheid van initiële genetische diversiteit. Hiervoor stelden we replicate monoklonale populaties bloot aan twee voedsel-behandelingen, één met voldoende en één met een tekort aan fosfor. Reeds na zes generaties konden we overerfbare adaptatie aan fosforlimitatie vaststellen bij die populaties met een historiek van blootstelling aan voedsel met een laag fosforgehalte. Dit was evenwel het geval bij slechts één van de twee geteste klones. Onze studie is één van de eerste die snelle overerfbare adaptatie aantoont aan omgevingsstress in populaties zonder initiële genetische variatie en suggereert dat nieuwe oorzaken van fenotypische variatie een belangrijke rol kunnen spelen bij het faciliteren van adaptatie bij populaties met een lage genetische diversiteit.

In de hoofdstukken $\mathbf{3}$ en $\mathbf{4}$ hebben we op een tijdschaal van meerdere generaties onderzocht in welke mate overerfbare veranderingen populaties in staat stellen om te gaan met stoichiometrische discrepanties. Niettemin kan de elementsamenstelling van het voedsel ook tijdens het leven van een consument sterk veranderen, waardoor diens vermogen hier snel op te kunnen reageren ook van groot belang is. Onderzoek bij hoge voedselconcentraties heeft aangetoond dat consumenten hun voedselopnamesnelheid aanpassen in functie van de elementsamenstelling van hun voedsel. Maar het effect van stoichiometrische discrepanties op de voedselopname langs een gradiënt van ecologisch relevante voedselconcentraties (meer bepaald de 'functionele respons') is tot zover nauwelijks of niet gekwantificeerd. Om het effect van stoichiometrische voedselkwaliteit op de functionele respons van primaire consumenten te bestuderen hebben we voor voedsel met verschillende element- en biochemische samenstelling de voedselopnamesnelheid bij verschillende voedseldichteden gemeten (Hoofdstuk 5). Bij hoge voedselconcentraties vertoonden consumenten met fosforarm voedsel verhoogde voedselopnamesnelheden vergeleken met fosforrijk voedsel. Dit was evenwel niet het geval bij lage voedselconcentraties. Dit kan verklaard worden doordat consumenten bij een gebrek aan fosfor meer voedsel tot zich gaan nemen met als doel de tekorten aan dit element te compenseren voor snelle groei. Dergelijke plastische responsen verdienen meer aandacht omdat ze de sterkte van trofische interacties kunnen beïnvloeden.

In Hoofdstuk $\mathbf{6}$ voorzie ik in een synthese van de bevindingen van de vorige hoofdstukken en kom ik op basis van de resultaten van mijn promotiewerk tot vier belangrijke besluiten. Ten eerste, populaties van primaire consumenten kunnen een substantiële hoeveelheid intraspecifieke genetische variatie in stoichiometrische kenmerken herbergen. Ten tweede, stoichiometrische discrepanties kunnen micro-evolutionaire trajecten beïnvloeden. Ten derde, fosforlimitatie kan tot cryptische evolutie leiden. Ten vierde, ecologische stoichiometrie kan gebruikt worden voor het voorspellen van fenotypische 
veranderingen maar het vereist de ontwikkeling van een meer algemeen theoretisch raamwerk. Dit proefschrift toont aan dat primaire consumenten de capaciteit hebben om te gaan met stoichiometrische discrepanties zowel via overerfbare als niet-overerfbare mechanismen. Ons vermogen om de respons van organismen ten aanzien van variatie in de relatieve beschikbaarheid van elementen te voorspellen kan baat hebben bij het verder incorporeren van realisme in ecologische stoichiometrie. 


\section{Acknowledgements}

As this chapter of my life comes to a close, I would like to thank those of you who have been part of the experience. Although the experimental aspect of my PhD may have been a bit turbulent, the support and encouragement of my family and friends has helped me navigate the journey. While the memories of the difficulties will fade, the people and the fun I had along the way will stay clear for a long time to come. I am so thankful for the abundance of personal and professional opportunities that my PhD has afforded me and will always look back fondly on my times in Wageningen.

I would first like to express my sincere gratitude to Steven for your supervision over the many, many, years of my PhD. We have been part of each other's lives during exciting and momentous changes including marriages, children, and the granting of tenure. I always enjoy our discussions, whether scientific, geopolitical, or the regarding the oddities of Dutch culture, and am eager to continue these over the years to come. Working with you has undoubtedly made me a better scientist and a stronger person. Thank you for the opportunity to be part of your group, and providing me the space to grow into the researcher that I am today. I look forward to our future collaborations and watching your lab and family grow. I would also like to thank my promotor Ellen for your guidance during my PhD, I wish you much happiness in your retirement. Finally, a big thank you to the research staff within the Aquatic Ecology department - Dedmer, Liesbeth, Wolf, and Lisette I always enjoyed and appreciated our engaging conversations about my project and other interesting topics.

My research would not have been possible without the assistance of others at the NIOO. Dennis, without you I would not be here today. Not only did you teach me everything I needed to know to run my experiments, but you were also a pillar of support. I have so many great memories of our conversations about life while building chemostats, cleaning chemostats, sampling chemostats, dismantling chemostats, swearing never to work with chemostats again... All jokes aside, you are an amazing friend and made me feel part of the community from my very first day. You approach the world with joy and kindness, making every person whose life you touch better. Nico, throughout my PhD you have been a great source of levity, humour, and advice. You were always there when I needed it, whether it be a last-minute analysis, to rescue a failing chemostat, or just for a heartfelt chat. I am sure every future workplace will feel a little lacking without your booming laughter filling the halls. Suzanne, you are such an amazing source of knowledge - talking to you always made me feel more confident that I actually knew what I was supposed to do. I still brag about the one time you complimented me about my pipetting 
accuracy - coming from someone as skilled in the lab as you are, I considered that the highest praise. Michaela, your work on the microsat analysis was a critical turning point in understanding the evolution experiment. Thank you for helping me with all my genetic analysis over the years. Erik, thank you for the elemental analysis you preformed over the years, helping with my crazy projects and being a great person to work with. I also would like to thank Iris who helped with elemental analysis. Finally, to all my great MBO students - Zarah, Nadine, Neils, and Erik, thank you for your hard work and dedication to this project.

I owe a great deal of thanks to the wonderful friends that I have made over the course of my PhD and I would like to start with those who were there at the very beginning. Paloma, thank you for showing me the ropes in the lab and introducing me to the many joys of Wageningen when I still mistakenly thought it was just a small farming town. You were my first true friend here and it is such a treat every time we get to be reunited. Lucy, thank you for all the wonderful adventures and thoughtful conversations - you provided an amazing sense of perspective. Gustavo and Marianneke you two bring such light and joy into my life. Thank you for your friendship over the years. Maíra, you are such a caring, generous soul, thank you for ensuring my time in Wageningen was lovingly sprinkled with music, dancing, delicious food, and a good drink (or two). Bart and Carlos, you two have been amazing friends, the moment I see you I always know my day is going to improve.

To Jan and Michiel, you are two of the loveliest individuals in the Netherlands. You worked together to hold me up and allowed for me to keep going when I no longer felt it was possible. Thank you for the deep meaningful conversations and the countless silly ones enjoyed over a piece of cake, ice cream, or a beer (or two).

To my adopted Wageningen family, Sui, Marta, Paolo, Thijs, and Antonella. I don't think it is possible for my writing to do justice to how much your friendship has meant to me over the Wageningen years and beyond. From nights out at De Zaaire and Shout, to beach vacations, to Whitney Houston karaoke in Paolo's apartment, to paddling canoes until your arms fell off, you all have shaped so many of my most precious memories of my PhD. I love you all immensely and you each deserve a paragraph of thanks for all the help and support you have given me. Instead, you will have to settle for a lifetime of my friendship, which unfortunately is non-negotiable.

Kadri and Julie, thanks for your support during the wrap up phase of my project and the zoom drinks and games over this last, strange, socially distant year, which has brought a little bit of normalcy to a very unusual time. Sarash, I am so glad to have a person as fun, welcoming, and thoughtful as you in my life. You are frankly 
the whole package, and during the end of this $\mathrm{PhD}$ you have ensured that I have had someone I could talk to and a glass of excellent wine in my had while doing so. Sam, you started off as my student, and you have become a great friend. You are such a joy to be around and are always ready to take me on an adventure. I would also like to thank the other strong, brilliant women researchers at the NIOO, Antica, Kelly, Ruth, Nori, and Marika. You have all been a source of strength, inspiration, and hilarity.

Libin and Wei, I was lucky to have you both as fellow Declerck Lab PhDs. We all somehow survived a PhD based on rotifer experimentation, and because of that we will be bonded for life. I want to thank you both for your assistance during experiments, and providing a positive and engaging working environment. Kong, Joost, and Casper, thanks for being great office mates. I really appreciated the scientific conversations and advice you provided. Lukas, although you were the last person to rotate into the office, I am so happy to have spent my last year at the $\mathrm{NIOO}$ with you by my side. I want to thank you and Ina for your friendship, fun times, and adventures. Peiyu, thank for all your encouragement, you are the most positive person I know. Just seeing you lights up my day. I would also like to thank the other students, PhDs, and postdocs within the Aquatic Ecology department that I crossed paths with during my time at NIOO - Soo, Susanne, Jing, Bart, Luuk, Annette, Laura, Karen, Alena, Mandy, Sven, Manqi, Maggie, and Hui you all made the department really enjoyable, have enriched my life in many ways.

I would also like to thank the pals who have been there for the closing phase of my $\mathrm{PhD}$ while I was in Switzerland. Blake, thank you for welcoming me into your group and making sure that I always was connect to the academic world. Ryan, Marvin, Leighton, and Angelina (plus Chase and Milly) you have been a great support network and have brought lots of fun to my time here in Switzerland. And of course, my darling Maria, you are the best adventure buddy a girl could ask for. I am so glad for all the coffees, laughs, and glasses of wine that we have shared. Thank you for the motivation you provided me in my final days. I would also like to thank Margo helping my vision of the thesis cover come to life - the time and care you put into making sure it was perfect was the best way to conclude my PhD.

To my amazing paranymphs, Tânia and Celia, you two mean so much to me. I am so happy that when I defend that it will be you (virtually) standing there by my side. Tânia, you welcomed me into your family when I was all alone in an unknown country, thousands of miles from my family and partner. You have taken care of me, and provided so much love and guidance (and wine) over the years and for that, I am endlessly grateful. I miss so much spending time with your family Martijn, Vincent and Sebastiaan you three have brought so much joy to my life and 
I look forward to every opportunity to see you. Celia you are my rock. The person who I always want to turn to whenever I have a challenge to face. You have always been there for me whenever I needed it, no questions asked. I am so lucky that we got to work together for my first thesis chapter, and I cannot thank you enough for the feedback that you have provided for the others. I could go on and on about how amazing you both are, the strength you have provided me, and how grateful I am to have you in my life, but for now I just want to thank you. For everything.

Thank you to my wonderful parents, who were so encouraging of my decision to move an ocean away to go have an adventure. I know these times apart have been tough (especially once trans-Atlantic travel became impossible) but I have always felt your love and support, and it has driven me to keep going and preserve through adversity. I also want to thank my absolute favourite sister Jillian, who ensured that I would make it to this day through occasional and well-timed reminders that she had more degrees than me. But all jokes aside, she has made me a better, kinder, more thoughtful person and always has my back in times of need. I would also like to thank Sue, Mike, Zac and Christina for all your love and support.

To my darling Cam. When I first began my PhD we were living a world apart $(13,458 \mathrm{~km}$ to be exact), only seeing each other during the unfortunately too infrequent visits and lots of facetime calls. Now, at the end, we have been given the opportunity to make up for the lost time, with the experience of living together during the last 15 months of lockdowns and social distancing. Throughout this entire time, whether near or far, you have been my constant champion. You have always been there whenever I needed someone talk to, a helping hand during experiments, or a shoulder to lean on. Your support whether it be through silly cards, surprise bouquets of flowers, the editing of drafts, or the cooking of dinner has been unwavering, and immensely appreciated. I want to thank you for all the sacrifices you have made over the last eleven years to get me to this place and to make this PhD happen. And so, my love, I dedicate this thesis to you. 


\section{About the Author}

Kimberley Lemmen was born on the 24th of September 1988 in Edmonton, Canada. She lived in Calgary, Alberta during her early years where she developed a passion for the outdoors by spending her summer vacations camping either in the Rocky Mountains or on Vancouver Island. In 2006 she started her BScH at Queen's University, Kingston, Ontario with the thought of eventually becoming a medical doctor. However, during a second-year field biology course at the Queen's University Biological Station she discovered her love of nature could be combined with her interest in biology and has been pursuing a career in ecology and evolutionary research ever since. Kimberley remained at Queen's for her MSc to investigate changes in salt tolerance of subarctic Daphnia populations under the supervision of Professor Shelley Arnott. In 2014 she travelled to Europe to begin a $\mathrm{PhD}$ at the Netherlands Institute of Ecology in Wageningen. Kimberley's primary research interest is in investigating microevolutionary trait shifts their influence ecosystem function. In the future she would like to explore the predictability of population level trait shifts to better forecast responses to environmental change.

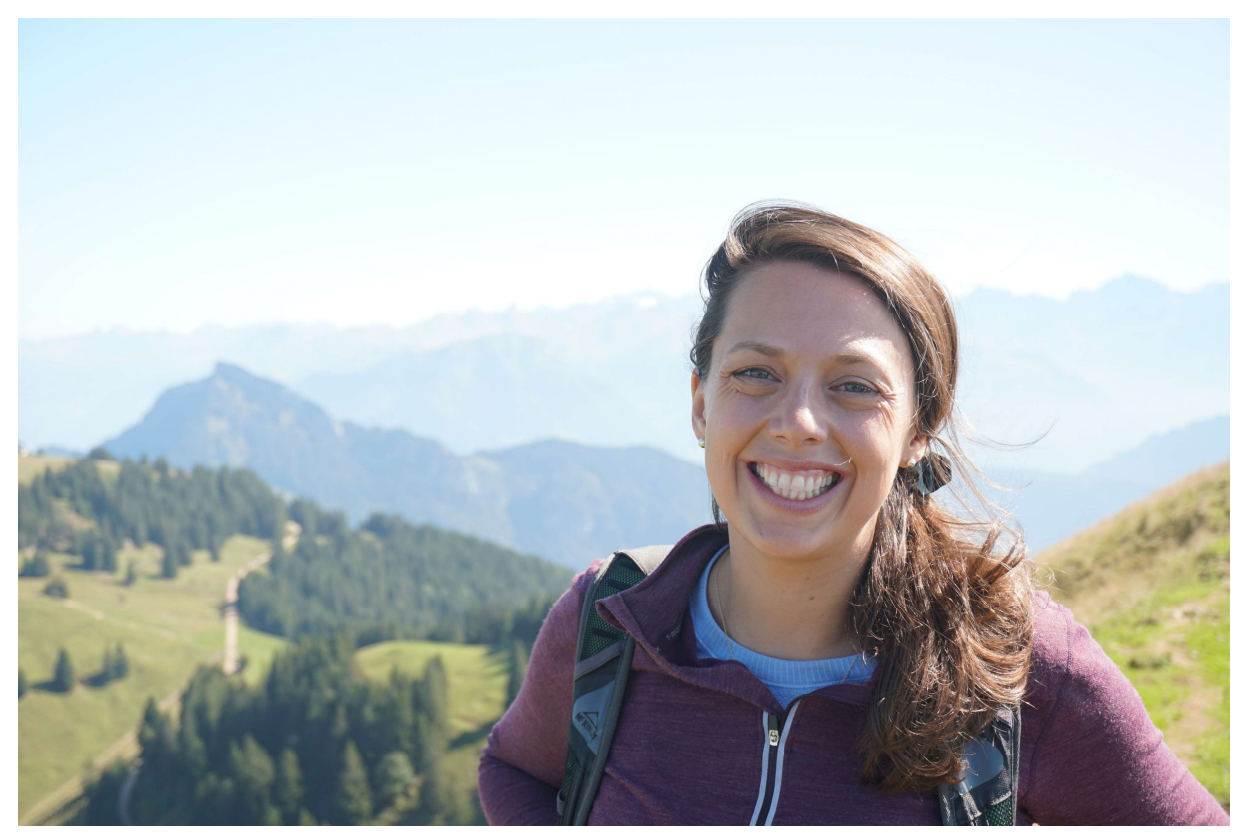




\section{Peer-reviewed Publications}

K. Lemmen, O. Butler, T. Koffel, S. Rudman, and C. Symons. 2019. Stoichiometric Traits Vary Widely Within Species: A Meta-analysis of Common Garden

Experiments. Frontiers in Ecology and Evolution, 7: 339.

W. Zhang, K. Lemmen, L. Zhou, S. Papakostas, and S.A.J. Declerck. 2019. Patterns of differentiation in the life history and demography of four recently described species of the Brachionus calyciflorus cryptic species complex. Freshwater Biology, 64: 1994-2005.

L. Zhou, K. Lemmen, W. Zhang, and S.A.J. Declerck. 2018. Direct and indirect effects of resource P-limitation differentially impact population growth, life history and body elemental composition of a zooplankton consumer. Frontiers in Microbiology, 9: 72.

S.A.J. Declerck, A. Malo, S. Diehl, D. Waasdorp, K. Lemmen, K. Proios, and S. Papakostas. 2015. Rapid adaptation of herbivore consumers to nutrient limitation: eco-evolutionary feedbacks to population demography and resource control. Ecology Letters, 18: 553-562.

T. Burg, S.A. Taylor, K. Lemmen, A.J. Gaston, and V.L. Friesen. 2014. Postglacial population differentiation potentially facilitated by a flexible migratory strategy in golden-crowned kinglets (Regulus satrapa). Canadian Journal of Zoology, 92: 163172.

\section{$\underline{\text { In Revision }}$}

K. Lemmen, K. Verhoeven, and S.A.J. Declerck. Experimental evidence of rapid heritable adaptation in the absence of initial standing genetic variation. Functional Ecology.

\section{Presentations at International Conferences}

Shallow Lakes (Virtual, 2021) Experimental evidence of rapid heritable adaptation in the absence of initial standing genetic variation.

Biology 20 Conference (Fribourg, Switzerland, 2020) Testing the growth rate hypothesis as a predictive framework for microevolutionary adaptation.

Experimental Evolution and Community Dynamics IV (Tvärminne Zoological Station, Finland, 2018) Variation in the microevolutionary response of a zooplankton consumer to selection for fast population growth under contrasting phosphorus availabilities. 
ASLO Summer Meeting (Victoria, Canada, 2018) Phosphorus availability determines rapid evolutionary response of zooplankton consumers to selection for fast growth.

DynaTrait conference (Hannover, Germany, 2017) Evidence for transgenerational adaptation to adverse resource stoichiometry in the absence of genetic variation.

Society of International Limnologists (Turin, Italy, 2016) Impact of Resource Stoichiometry on the Herbivore Brachionus calyciflorus.

Conference on Biological Stoichiometry (Peterbourgh, Canada, 2015) Functional response of the herbivore Brachionus calyciflorus across a stoichiometric gradient.

Participation in International Workshops

Woodstoich IV. 2019. Flathead Lake Biological Station, Montana. Group Leader of Eco-Evo Dynamics \& Stoichiometry working group.

\section{Conference sessions organized}

SS12 Understanding (mal)adaptations to environmental change. ASLO Summer Meeting 2021 (Virtual) Co-organized with Celia Symons (UC Irvine)

Awards

First Place PhD Oral Presentation. Netherlands Annual Ecology Meeting (2018) 
PE\&RC Training and Education

\section{Statement}

With the training and education activities listed below the PhD candidate has complied with the requirements set by the C.T. de Wit Graduate School for Production Ecology and Resource Conservation (PE\&RC) which comprises a minimum total of 32 ECTS ( $=22$ weeks of activities)

\section{Review of literature (6 ECTS)}

Stoichiometric imbalance and possible impacts on eco-evolutionary dynamics

Post-graduate courses (5 ECTS)

Mixed Linear Models, PE\&RC (2017)

Generalized Linear Models, PE\&RC (2017)

Dynamic Models in R: Programming, parameter estimation and model selection, PE\&RC (2017)

Eco-Evo Dynamics \& Stoichiometry, Flathead Biological Station, University of Montana (2019)

Competence strengthening / skills courses (1.5 ETCS)

The Essentials of Scientific Writing and Presenting, WGS (2018)

PhD \& Postdoc career development day; KNAW (2018)

PE\&RC annual meetings, seminars and the PE\&RC Weekend (1.5 ETCS)

PE\&RC First years weekend (2014)

PE\&RC Day $(2015,2018)$

Discussion groups / local seminars or scientific meetings (9.4 ETCS)

NIOO Seminar \& scientific meetings (2014-2018)

Netherlands Annual Ecology Meeting (Lunteren, 2015, 2016, 2018)

Netherlands Society for Evolutionary Biology $(2018,2019)$

Eco-Evolutionary NIOO Theme group meetings $(2018,2019)$

EAWAG Fish Ecology and Evolution Seminar Series (2020)

182 | Thesis summary \& About the author

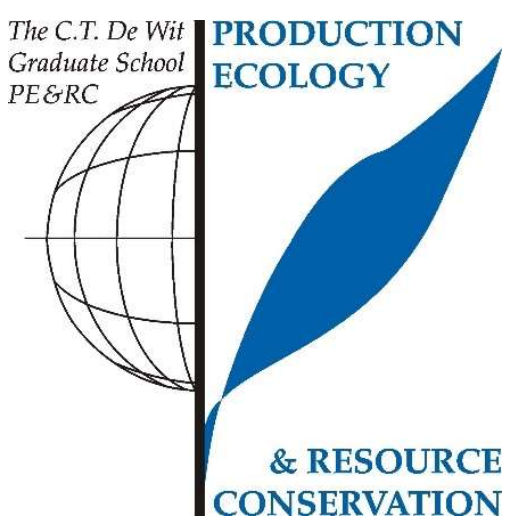




\section{International symposia, workshops, and conferences (14.8 ETCS)}

Conference on Biological Stoichiometry (Peterborough, Canada, 2015) Functional response of the herbivore Brachionus calyciflorus across a stoichiometric gradient. Oral Presentation.

Society of International Limnologists (Turin, Italy, 2016) Impact of Resource Stoichiometry on the Herbivore Brachionus calyciflorus. Oral Presentation.

DynaTrait conference (Hannover, Germany, 2017) Evidence for transgenerational adaptation to adverse resource stoichiometry in the absence of genetic variation. Oral Presentation.

ASLO Summer Meeting (Victoria, Canada, 2018) Phosphorus availability determines rapid evolutionary response of zooplankton consumers to selection for fast growth. Oral Presentation.

Experimental Evolution and Community Dynamics IV (Tvärminne Zoological Station, Finland, 2018) Variation in the microevolutionary response of a zooplankton consumer to selection for fast population growth under contrasting phosphorus availabilities. Oral Presentation.

Biology 20 Conference (Fribourg, Switzerland, 2020) Testing the growth rate hypothesis as a predictive framework for microevolutionary adaptation. Oral Presentation.

Shallow Lakes (Virtual, 2021) Experimental evidence of rapid heritable adaptation in the absence of initial standing genetic variation. Oral Presentation.

\section{Lecturing/Supervision of practicals/tutorials (1.8 ECTS)}

Aquatic ecology bachelor course, zooplankton practical; University of Utrecht, NIOO $(2016,2018)$

\section{Supervision of MSc student (3 ECTS)}

Role of functional response modifications in the microevolutionary adaptation of rotifer populations to phosphorus limitation. 